University of Louisville

ThinkIR: The University of Louisville's Institutional Repository

Electronic Theses and Dissertations

$12-2020$

\title{
Investigating the disorder and compaction of designed Minielastin using nuclear magnetic resonance.
}

Ma Faye Charmagne Aquino Carvajal

University of Louisville

Follow this and additional works at: https://ir.library.louisville.edu/etd

Part of the Biochemistry Commons

\section{Recommended Citation}

Carvajal, Ma Faye Charmagne Aquino, "Investigating the disorder and compaction of designed Minielastin using nuclear magnetic resonance." (2020). Electronic Theses and Dissertations. Paper 3532.

https://doi.org/10.18297/etd/3532

This Doctoral Dissertation is brought to you for free and open access by ThinkIR: The University of Louisville's Institutional Repository. It has been accepted for inclusion in Electronic Theses and Dissertations by an authorized administrator of ThinkIR: The University of Louisville's Institutional Repository. This title appears here courtesy of the author, who has retained all other copyrights. For more information, please contact thinkir@louisville.edu. 
INVESTIGATING THE DISORDER AND COMPACTION OF DESIGNED MINIELASTINS USING NUCLEAR MAGNETIC RESONANCE

By

Ma. Faye Charmagne Aquino Carvajal

B.S., University of the Philippines, 2010

M.S., University of Louisville, 2018

\begin{abstract}
A Dissertation
Submitted to the Faculty of the

College of Arts and Sciences of the University of Louisville

in Partial Fulfillment of the Requirements

for the Degree of
\end{abstract}

Doctor of Philosophy

in Chemistry

Department of Chemistry

University of Louisville

Louisville, KY

December 2020 
Copyright 2020 by Ma. Faye Charmagne A. Carvajal

All rights reserved 

INVESTIGATING THE DISORDER AND COMPACTION OF DESIGNED MINIELASTINS USING NUCLEAR MAGNETIC RESONANCE

By

Ma Faye Charmagne Aquino Carvajal

B.S., University of the Philippines, 2010

M.S., University of Louisville, 2018

A Dissertation Approved on

November 30, 2020

By the following Dissertation Committee

Dr. Richard J. Wittebort

Dr. Donghan Lee

Dr. Muriel C. Maurer

Dr. Eugene G. Mueller

Dr. Xiang Zhang 


\section{DEDICATION}

This dissertation is dedicated to my parents,

Mr. Henry C. Carvajal and Mrs. Remedios A. Carvajal

who supported me all the way,

and to my husband,

Caleb Aaron Calvary

for not giving up. 


\section{ACKNOWLEDGMENTS}

I am deeply indebted to my mentor, Dr. Richard Wittebort. Despite all the major hurdles in life, you have provided me with unparalleled support and guidance to finish my dissertation.

I am extremely grateful to my Dissertation committee, Dr. Donghan Lee, Dr. Muriel Maurer, Dr. Eugene Mueller, and Dr. Xiang Zhang for your helpful advice and constructive criticisms.

I would like to extend my deepest gratitude to our collaborators in CCNY, Dr. Ronald Koder, Jonathan Preston and Dr. James Aramini for providing the training and the facilities necessary for these research projects. Also, to our collaborators in UofLHSC, Dr. Mike Sabo and Dr. William Dean, thank you for your valuable inputs and for sharing your expertise.

Special thanks to Sherry Nalley, for the enormous amount of assistance. I appreciate all the help you've given me.

I am thankful to the best lab buddy, Nour Jamhawi, for the constant push when motivation is lacking, for cheering me up when I'm down and for always being there.

To all the friends I've made during my stay in UofL, Cipriana Prepeliuc, Nina Saraei, Amareshwari Konutham, Nick Vishnosky, Jason Young, Dino Ablan, Richard Lumata, Hari Nambiar, Emily Kempfer-Robertson, Christine Phipps, Kritika Bajaj, Chinmay Potnis, Dillon Hofsommer, Peter Armstrong, thank you for the company, holiday get-togethers and game nights. You made my graduate school life bearable and less stressful. Y'all made Louisville feel like home away from home.

Special thanks to Razel Ventura for the emotional and mental support when I need it the most. Also, my gratitude to Dustin Woods for the intellectual and personal conversations that provided me with a different perspective.

My utmost gratitude to my parents and my siblings, for the love and understanding, - to my mom who keeps on believing in me, for pushing me to do my best and not to give up.

I am grateful to my in-laws, Mark and Nancy Calvary and to late grandma, Eleanor Calvary, for the unwavering support and prayers.

Lastly, to my loving husband, Caleb Calvary, who has been with me throughout this journey, I couldn't have done this without you. 


\title{
ABSTRACT \\ INVESTIGATING THE DISORDER AND COMPACTION OF DESIGNED MINIELASTINS USING NUCLEAR MAGNETIC RESONANCE
}

\author{
Ma. Faye Charmagne A. Carvajal
}

November 30, 2020

Minielastins are elastin-based proteins with alternating hydrophobic and crosslink modules similar to tropoelastin. Tropoelastin is the $\sim 70 \mathrm{kDa}$ soluble monomeric precursor of elastin. The extracellular matrix protein, elastin provides elasticity to tissues and organs such as lungs, arteries and ligaments. The elastic properties of natural elastin are believed to be entropic in origin. In vivo, the elastin matrix is approximately $50 \%$ water by weight. Without water, elastin is brittle and hard.

Minielastins, like tropoelastin, undergo a liquid-liquid phase transition upon an increase in temperature. Factors such as hydrophobicity, chain length and concentration affect the coacervation temperature, $T_{c}$. The coacervation temperature were modulated by changing the number of hydrophobic repeats and the length of cross-link modules. Each hydrophobic repeat, VPGVGG and APGVGV, decreases $\mathrm{T}_{\mathrm{c}}$ by 1.7 and $1.5{ }^{\circ} \mathrm{C}$, respectively. Also, increasing the temperature and pressure causes the hydrodynamic radii of minielastins to shrink, similar to other disordered proteins. 
Elastin-like biomaterials exhibit a great potential in tissue engineering and drug delivery. Therefore, it is important, for a wide array of applications, to understand the relationship between protein sequence, structure and mechanical properties of elastin biomaterials. To obtain residue specific information using nuclear magnetic resonance (NMR) spectroscopy, simplified hydrophobic and cross-link modules were designed based on the predominant 6-residue repeats found in tropoelastin exon 20 (VPGVGG) and 24 (APGVGV) hydrophobic modules and the consensus cross-link motif, $\mathrm{A}_{4 / 5} \mathrm{KA}_{2 / 3} \mathrm{~K}$, found in the natural elastin.

In this study, the hydrophobic modules, responsible for the self-assembly of elastin-like proteins, were found to be highly disordered. Also, the cross-link modules are disordered, but when flanked by hydrophobic modules they are weakly $\alpha$-helical. These conclusions are supported by analysis of complete assignment of backbone ${ }^{1} \mathrm{H},{ }^{13} \mathrm{C}$ and ${ }^{15} \mathrm{~N}$ chemical shifts and ${ }^{15} \mathrm{~N}$ spin relaxation measurements $\left(R_{1}, R_{2}\right.$ and NOE) with spectral density modeling. These results show that the amplitude of dynamics in the hydrophobic modules approach that of a flexible polymer chain with chain dynamics on two timescales, $\sim 1-2$ ns and $\sim 30-80$ ps. Well-ordered regions were not found. Finally, the ability of minielastins to form insoluble cross-linked products have proved its potential as an elastic biomaterial. 


\section{TABLE OF CONTENTS}

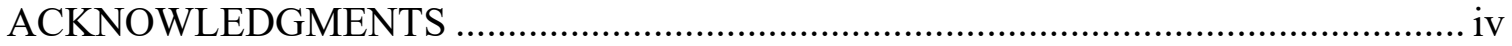

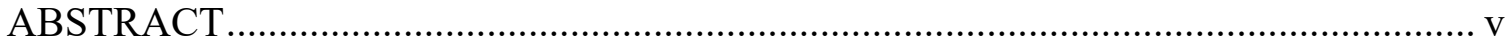

LIST OF FIGURES ……………………………….............................................

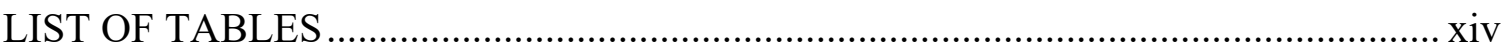

CHAPTER 1. Minielastins are intrinsically disordered proteins ........................................ 1

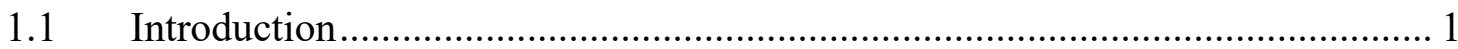

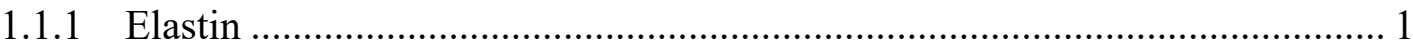

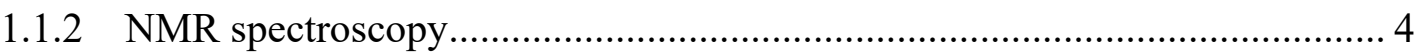

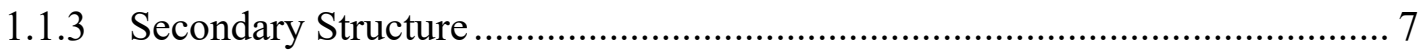

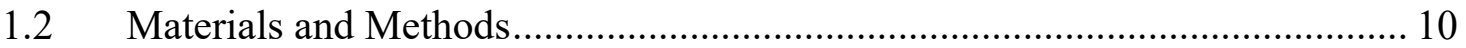

1.2.1 Protein Expression .............................................................................. 10

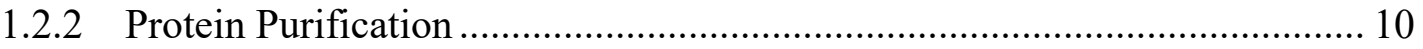

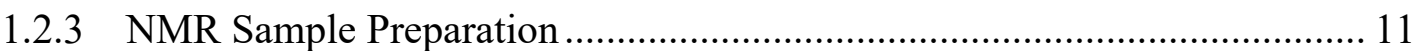

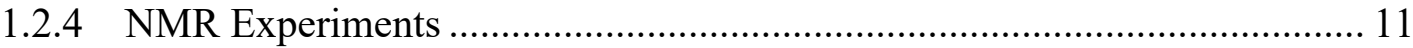

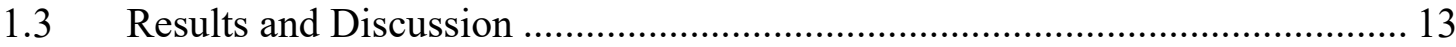

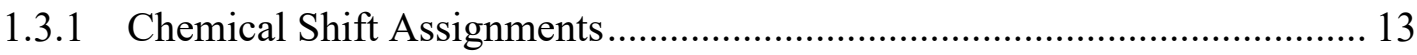

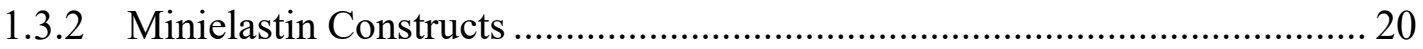

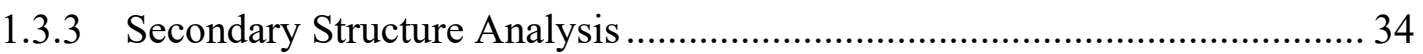

CHAPTER 2. Minielastins are more compact than typical IDPs and undergo liquidliquid phase transition like tropoelastin ......................................................................... 44 


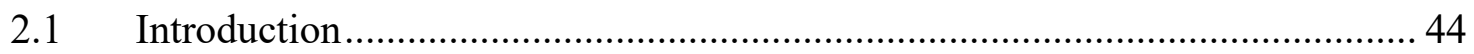

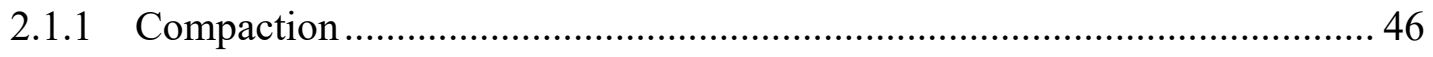

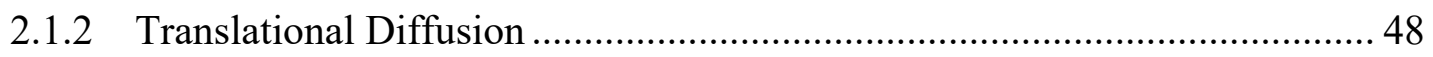

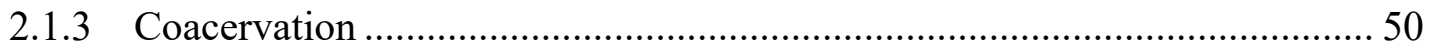

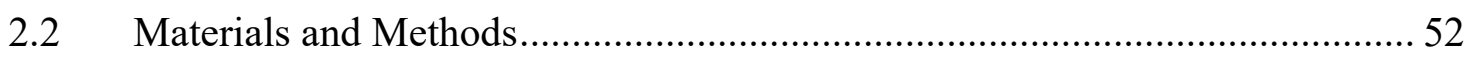

2.2.1 Pulsed Field Gradient NMR ……………………................................. 52

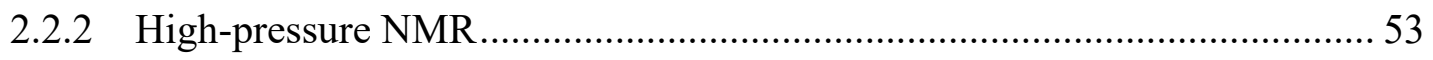

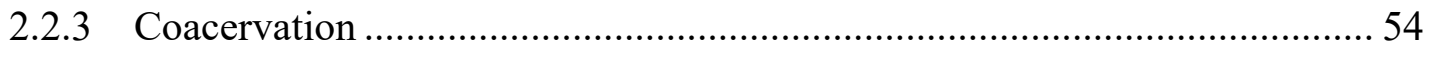

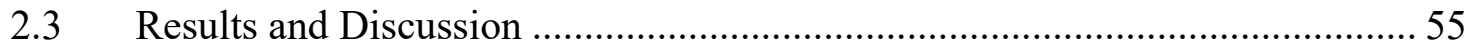

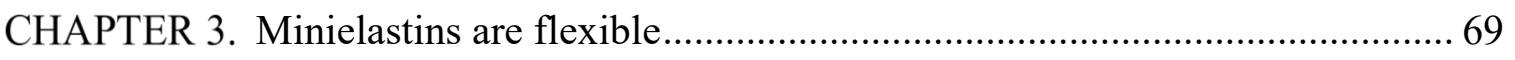

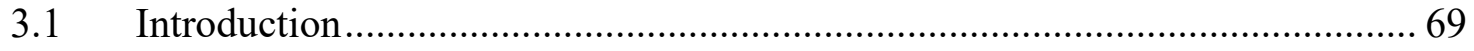

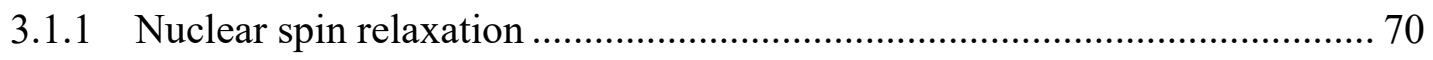

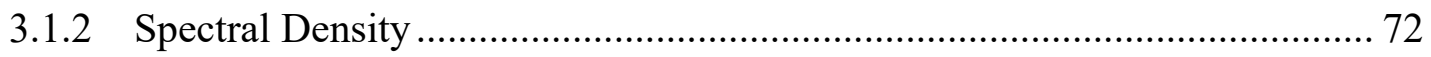

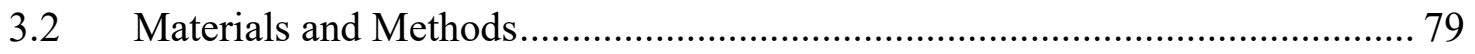

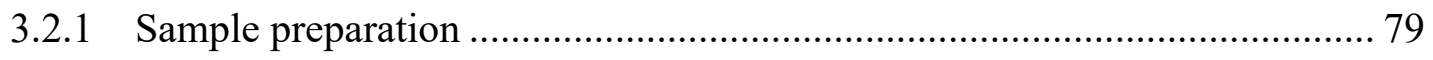

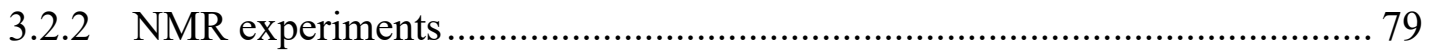

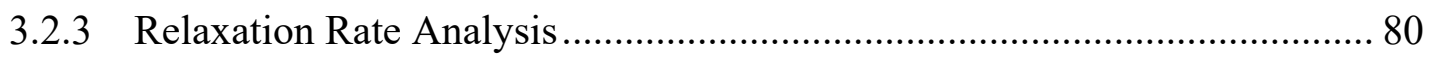

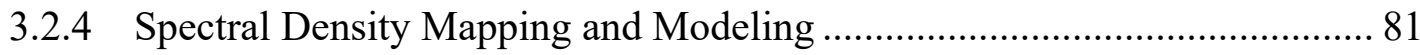

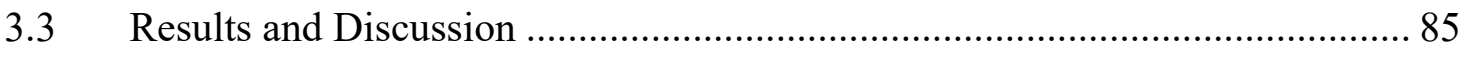

CHAPTER 4. Minielastin forms an insoluble cross-linked product with genipin........... 98

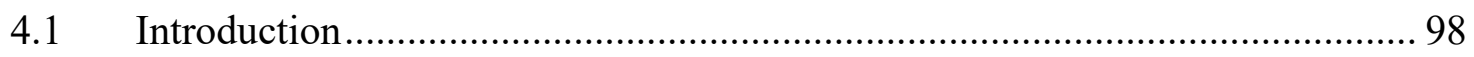

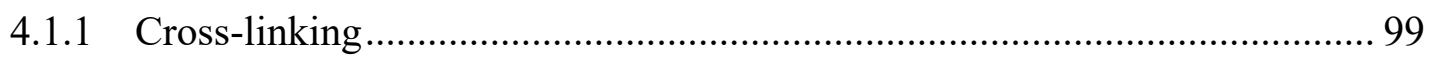

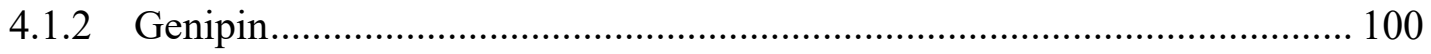




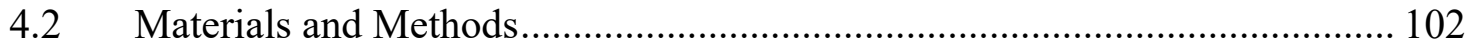

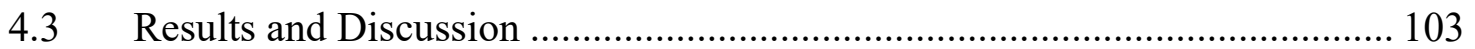

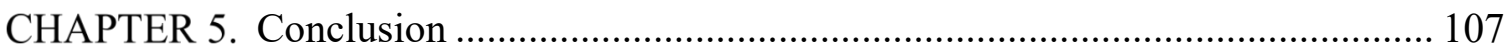

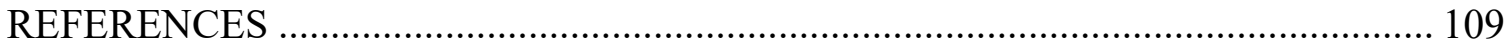

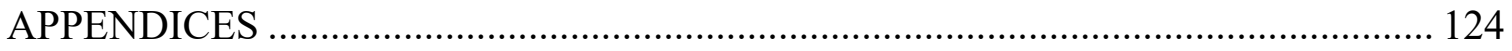

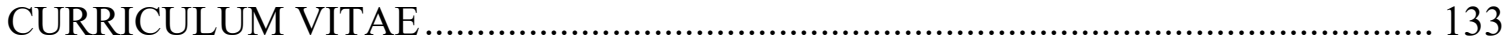




\section{LIST OF FIGURES}

Figure 1.1. Human tropoelastin sequence from cDNA divided into different exons with stars depicting exons subjected to alternative splicing (adapted from Bashir et al. ${ }^{4}$ ). ...... 2

Figure 1.2 Formation of the bi-,tri-, and tetrafunctional cross-linker in elastin adapted from Vrhovski et al. ${ }^{9}$

Figure 1.3. Spin system of the peptide backbone and the size of the ${ }^{1} J$ and ${ }^{2} J$ coupling constants that are used for magnetization transfer in ${ }^{13} \mathrm{C}$ and ${ }^{15} \mathrm{~N}$-labelled proteins.

Figure 1.4. NMR strategy for structure elucidation of minielastins features $2 \mathrm{D}$ and $3 \mathrm{D}$ multinuclear experiments.

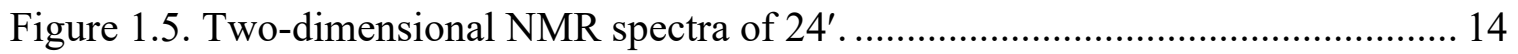

Figure 1.6 NOESY (red) overlaid with TOCSY (blue) fingerprint (top) and amide (bottom) region to differentiate peaks from neighboring residues. .............................. 15

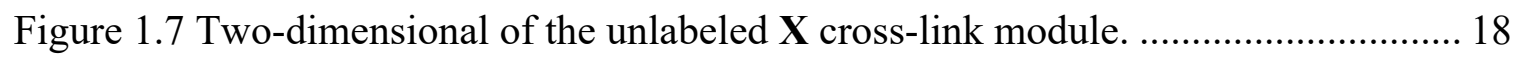

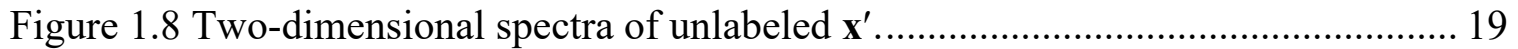

Figure $1.9{ }^{15} \mathrm{~N}-{ }^{1} \mathrm{H}$ HSQC of $24 \mathrm{x}^{\prime}$ (red), 24' (black) and $\mathrm{x}^{\prime}$ (blue). All proton shifts are within a 1 ppm range. Assignments were taken from $24^{\prime}$ as it matches the peaks in $24 \mathrm{x}^{\prime}$

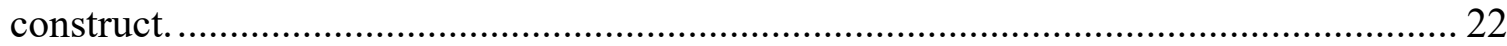

Figure 1.10 Strips from the CBCACONH spectrum (blue) overlaid with those from CBCANH..

Figure 1.11 Sequential assignment of the cross-link module using strips from the HNCO (green) and HNCACO (red) spectra.

Figure 1.12 Overlaid ${ }^{15} \mathrm{~N}-{ }^{1} \mathrm{H}$ HSQC of $20 \mathrm{x}^{\prime}$ (black) and $24 \mathrm{x}^{\prime}$ (red). 26

Figure $1.13{ }^{15} \mathrm{~N}$-edited TOCSY-HSQC (red) paired with ${ }^{15} \mathrm{~N}$-edited NOESY-HSQC (blue) of ${ }^{15} \mathrm{~N}$-labeled $20 \mathrm{x}^{\prime}$. 
Figure 1.14 Overlaid spectra of the ${ }^{15} \mathrm{~N}-{ }^{1} \mathrm{H}$ HSQC of 202424 (red), 2020 (black) and 24' (blue).

Figure $1.15 \mathrm{CBCANH} / \mathrm{CBCACONH}$ (top) and $\mathrm{HNCO} / \mathrm{HNCACO}$ (bottom) of the first four residues of the cross-link modules of 2020 . 31

Figure 1.16 (a) $\mathrm{HNCO} / \mathrm{HNCACO}$ of GVGT (b) CBCANH/CBCACONH and (c) HNN of QFG of the cross-link modules of 2020 .

Figure $1.17 \mathrm{HNCO} / \mathrm{HNCACO}$ of alanine-rich cross-link module used to very resonance assignments. $\mathrm{HNCO}$ peaks (green) originates from $\mathrm{CO} i-1$ whereas $\mathrm{HNCACO}$ peaks (red) are from $\mathrm{CO}$ of $i$ and $i-1$ residues.

Figure 1.18 Secondary shift analysis of the hydrophobic modules; top panel for $24^{\prime}$ and bottom panel for $20^{\prime}$ in 2020 . 34

Figure 1.19 Secondary shift analysis of the free cross-linking peptides $\mathrm{X}$ and $\mathrm{x}^{\prime}$ (gray) versus the cross-linking module in 2020 and $24 x^{\prime}$ (red). 35

Figure 1.20 (a) ${ }^{15} \mathrm{~N}$-edited NOESY-HSQC (red) overlaid with ${ }^{15} \mathrm{~N}$-edited TOCSY-HSQC of $24^{\prime}$ in $24 x^{\prime}$. (b) NOE ratios of sequential and intraresidue $\alpha \mathrm{N}$ of $24^{\prime}$ found in four different constructs

Figure $1.21{ }^{15} \mathrm{~N}$-edited NOESY-HSQC of the cross-link residues showing NOE contacts between amide protons. 38

Figure 1.22 NMR spectra of $24 \mathrm{x}^{\prime}$ at different temperatures. 40

Figure 1.23 Circular dichroism spectra of (a) 202424 (red) and 2020 (blue) with free 24'

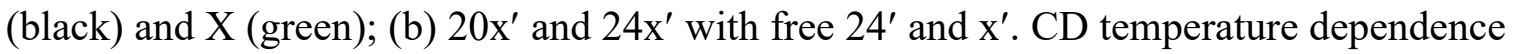
of (c) 202424 and (d) $24 \mathrm{x}^{\prime}$ shows minimal change in intensity at 200 and $220 \mathrm{~nm}$. 42

Figure 2.1 The total volume change $\left(\Delta \mathrm{V}_{\text {tot }}\right)$ associated with unfolding of natively folded proteins depend on the balance between the change in void volume $\left(\Delta \mathrm{V}_{\text {void }}\right)$ and the change in hydration volume $\left(\Delta \mathrm{V}_{\text {hyd }}\right)$. 47

Figure 2.2 (a) Pulsed Field Gradient Spin Echo (adapted from Johnson, 199977) and representative magnitude of signal in each step of the pulse sequence. (b) Plot of signal intensity with increasing field strength is a gaussian curve.

Figure 2.3 Normalized signal intensities were plotted versus the gradient strength at a) different temperatures and b) different trials at $25{ }^{\circ} \mathrm{C}$ of 202424 shows the reversibility and reproducibility of the diffusion experiments. 53

Figure 2.4 Schematic of the coacervation experiment for $20 \mathrm{x}^{\prime}$ and $24 \mathrm{x}^{\prime}$. 54 
Figure 2.5 Hydrodynamic radii of minielastins at $25^{\circ} \mathrm{C}$ (filled circles) with calculated $R_{\mathrm{H}}$

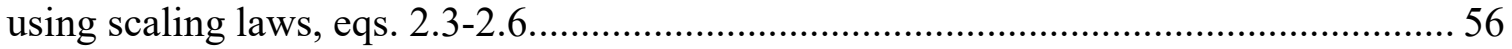

Figure 2.6 Temperature-dependence of hydrodynamic radii of minielastins. Slopes of the $\mathrm{R}_{\mathrm{H}}$ versus temperature plot are shown in the inset and reported in Table 2.1. 57

Figure 2.7 Dependence of coacervation temperature with the average per residue hydrophobicity of minielastin constructs using Kyte-Doolittle hydropathy indices. ....... 62

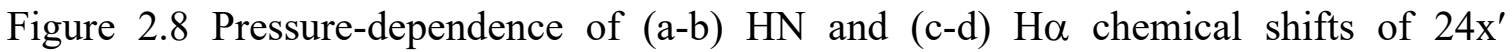
measured at 20 to 2500 bar with second order coefficients reported in Table 2.3........... 64

Figure 2.9 Change in hydrodynamic radii of $17 \mathrm{APG}, 20242424$ and $24 \mathrm{x}^{\prime}$ constructs with pressure ranges from 20 to 2500 bar. 67

Figure 3.1 Inversion recovery pulse sequence to measure $R_{1} .180^{\circ}$ pulse inverts the $\alpha$ and $\beta$ populations. As time delay, $\tau$, increases the more time the spins have to relax back to thermal equilibrium. 71

Figure 3.2 Spin echo pulse sequence to measure $R_{2}$ relaxation rate. Spin coherence is lost with increasing time delay $\tau$. 72

Figure 3.3 Nuclear spin relaxation observables (a) longitudinal relaxation rate $R_{1}$ (b) transverse relaxation rate $R_{2}$ of residues $\mathrm{V}(6,24)$ in red and $\mathrm{A}\left(5, \mathrm{x}^{\prime}\right)$ in gray and (c) [ $\left.{ }^{1} \mathrm{H}\right]$ ${ }^{15} \mathrm{~N}$ heteronuclear steady state NOE of $24 \mathrm{x}^{\prime}$ with and without proton saturation in green and red spectrum, respectively. 80

Figure 3.4 NMR spin relaxation data $R_{1}, R_{2}$ and heteronuclear NOE at 500 (blue), 600 $\mathrm{MHz}$ (magenta) and $800 \mathrm{MHz}$ (red) for each residue in $24 \mathrm{x}^{\prime}$. 86

Figure 3.5 Spectral density calculated from the NMR spin relaxation data at a) $J(0)$ averaged at three magnetic fields, b) $J(\omega \mathrm{N})$ and $J(0.87 \omega \mathrm{H})$ obtained from 500 (open square), 600 (open triangle) and $800 \mathrm{MHz}$ (open circle) magnetic fields. 88

Figure 3.6 The average spectral density functions for hydrophobic (red) and cross-link (gray) were sampled at $0, \omega \mathrm{N}, 0.87 \omega \mathrm{H}, 0.921 \omega \mathrm{H}$, and $0.955 \omega \mathrm{H}$ at 500,600 and 800 MHz. ${ }^{137}$ 89

Figure 3.7 Fit parameters of A $\left(1,24^{\prime}\right)$ with calculated relaxation values within two standard deviation of the experimental values obtained from sum of Lorentzian with $\mathrm{k}=$ 3. $\chi^{2}$ values indicated by the colormap.

91

Figure 3.8 Fit parameters obtained for sum of Lorentzian model with $\mathrm{k}=3$ (red circles) and extended Lipari-Szabo with Gaussian distribution (blue squares) 94 
Figure 3.9 ELS parameters at $\tau_{\mathrm{M}}$ equal to $4 \mathrm{~ns}$ (yellow), $19 \mathrm{~ns}$ (red), $40 \mathrm{~ns}$ (blue) and $\tau \mathrm{M}$ with gaussian distribution (black). In top left are the $\mathrm{S}_{\mathrm{s}}{ }^{2}$ and $\mathrm{S}_{\mathrm{f}}{ }^{2}$ values given that $\mathrm{S}^{2}=\mathrm{S}_{\mathrm{s}}{ }^{2}$

$\mathrm{St}^{2}$. 95

Figure 3.10 Spectral density function of $\mathrm{A}(1,24)$ and $\mathrm{A}\left(6, \mathrm{x}^{\prime}\right)$ calculated from experimental relaxation data using method 3. Spectral density function with fit parameters calculated using eq. 3.6 are shown in dashed lines.

Figure 4.1 Lysyl oxidase catalyzes the oxidative deamination of lysine side chain and yields allysine, hydrogen peroxide and ammonia. ${ }^{155}$ 99

Figure 4.2 Several cross-linkers used for tropoelastin and ELP cross-linking. Bis(sulfosuccinimidyl)suberate ${ }^{157}$ is a bifunctional cross-linker whereas genipin, ${ }^{15}$ lysyl oxidase $^{158}$ and $\mathrm{PQQ}^{15,159}$ yields bi-, tri- and tetrafunctional cross-links. 100

Figure 4.3 Reaction of genipin with primary amines yields a) yellow and b) brownish-red intermediates. Proposed cross-linked products based on the mechanisms of c) Butler and coworkers ${ }^{162}$ and d-e) Touyama and coworkers. ${ }^{163-164}$ 101

Figure 4.4 Reaction progression of genipin with BOC-glycine, glycine and lysine amino acids in $\mathrm{pH} 6$ phosphate buffer and in $\mathrm{pH} 8$ borate buffer. The formation of colored intermediates and the final water-soluble blue pigment. 104

Figure 4.5 Progression of the cross-linking process. 105 


\section{LIST OF TABLES}

Table 1.1 Chemical shift assignment of 24' obtained from 2D experiments................... 16

Table 1.2 Names, number of residues, molecular weights and sequences of the

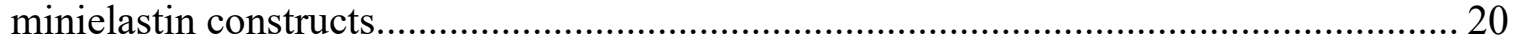

Table 2.1 Hydrodynamic radii from pulsed field gradient NMR at $20{ }^{\circ} \mathrm{C}$ and calculated

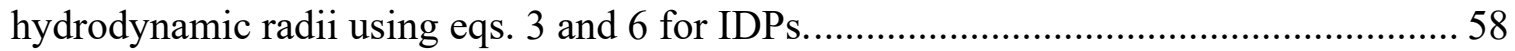

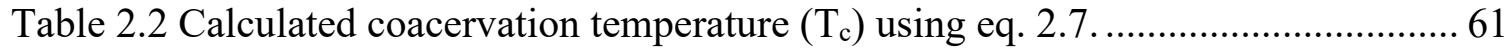

Table 2.3 Coefficients of second order polynomial fitting of proton chemical shifts versus

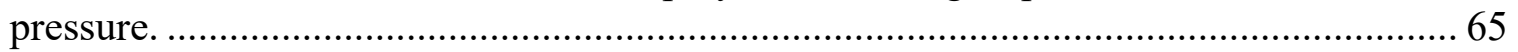

Table 3.1 Spectral density approaches with adjustable parameters used to fit the

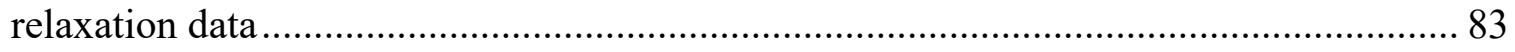

Table 3.2 Fit parameters obtained from sum of Lorentzian with $\mathrm{k}=2$ and 3 at $\chi^{2} \min \ldots . . .90$ 


\section{CHAPTER 1. MINIELASTINS ARE INTRINSICALLY DISORDERED}

\section{PROTEINS}

\subsection{Introduction}

\subsubsection{Elastin}

Elastin is an insoluble protein found in the extracellular matrix of the cell. It is responsible for the elasticity of tissues and organs such as ligaments, arteries, lungs and skin. The mechanism of elasticity is known to be entropic, i.e., spontaneous recoil is driven by the increase in entropy upon relaxing from stretch. Hydrated elastin is elastic and flexible but dry elastin is hard and brittle. Thus, hydration plays an important role in the protein's elasticity. ${ }^{1}$

Mature elastin is composed of networks of cross-linked tropoelastin, a soluble monomer that has been widely studied since it was first isolated from copper-deficient animals. $^{2}$ Tropoelastin is a $\sim 70-\mathrm{kDa}$ protein with alternating hydrophobic and crosslinking modules coded by 34 exons, Figure 1.1. Hydrophobic domains are rich in glycine, alanine, proline and valine with predominant GXG, GGX and PGX motifs where $\mathrm{X}$ is $\mathrm{V}, \mathrm{A}, \mathrm{I}$ or L. Whereas hydrophobic modules contain mainly aliphatic residues, KA and KP cross-linking domains contain polyalanyl sequences followed by KAAK or KAAAK. Exon 1 contains the signaling sequence whereas exon 36 translates to the positively charged RKRK C-termini sequence. The elastin gene undergoes alternative splicing resulting to different isoforms of tropoelastin. ${ }^{3}$ 


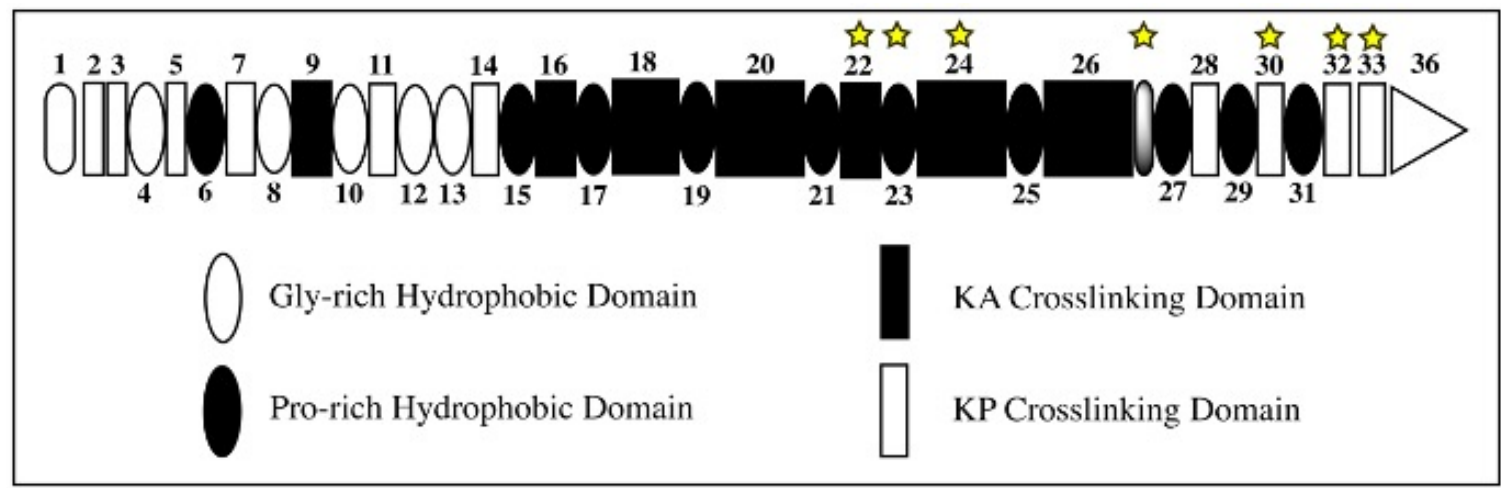

\begin{tabular}{|c|c|c|c|}
\hline Exon \# & Sequence & Exon \# & Sequence \\
\hline 2 & GVPGAIPGGCPGGVFYP & 4 & GALGPGGKPLKPV \\
\hline 3 & GAGLGALGG & 6 & $\begin{array}{l}\text { GLGAFPAVTFPGALVPGGVADAAAAY } \\
\text { KAAKA }\end{array}$ \\
\hline 5 & PGGLAGAGLGA & 8 & GAVVPQPGAGVKPGKVP \\
\hline 7 & GAGLGGVPGVGGLGVSA & 10 & RFPGVGVLPGVPTGAGVKPKAP \\
\hline 9 & GVGLPGVYPGGVLPGA & 12 & GVGPFGGPQPGVPLGYPIKAPKLP \\
\hline 11 & GVGGAFAGIP & 13 & GGYGLPYTTGKLPY \\
\hline 14 & GYGPGGVAGAAGKAGYPTGT & 15 & GVGPQAAAAAAAKAAAKF \\
\hline 16 & GAGAAGVLPGVGGAGVPGVPGAIPGIGGIA & 17 & GVGTPAAAAAAAAAAKAAKY \\
\hline 18 & $\begin{array}{l}\text { GAAAGLVPGGPGFGPGVVGVPGAGVPGVGVPGAGI } \\
\text { PVVPGAGIPGAAVP }\end{array}$ & 19 & GVVSPEAAAKAAAKAAKY \\
\hline 20 & $\begin{array}{l}\text { GARPGVGVGGIPTYGVGAGGFPGFGVGVGGIPGVA } \\
\text { GVPSVGGVPGVGGVPGVGIS }\end{array}$ & 21 & PEAQAAAAAKAAKY \\
\hline 22 & GAAGAGVLGGLVPGPQAAVPGVPGTGGVP & 23 & GVGTPAAAAAKAAAKAAQF \\
\hline 24 & $\begin{array}{l}\text { GLVPGVGVAPGVGVAPGVGVAPGVGLAPGVGVAPG } \\
\text { VGVAPGVGVAPGI }\end{array}$ & 25 & GPGGVAAAAKSAAKVAAKAQL \\
\hline 26 & $\begin{array}{l}\text { RAAAGLGAGIPGLGVGVGVPGLGVGAGVPGLGVG } \\
\text { AGVPGFGA }\end{array}$ & 27 & PGALAAAKAAKY \\
\hline 28 & GAAVPGVLGGLGALGGVGIPGGVV & 29 & GAGPAAAAAAAKAAAKAAQF \\
\hline 30 & GLVGAAGLGGLGVGGLGVPGVGGLG & 31 & GIPPAAAAKAAKY \\
\hline 32 & GAAGLGGVLGGAGQFPLG & & \\
\hline 33 & GVAARPGFGLSPIFP & & \\
\hline 36 & GGACLGKACGRKRK & & \\
\hline
\end{tabular}

Figure 1.1. Human tropoelastin sequence from cDNA divided into different exons with stars depicting exons subjected to alternative splicing (adapted from Bashir et al. ${ }^{4}$ ). The predominant hydrophobic (red) and cross-link (blue) repeats highlighted were used in designing minielastin constructs.

Mature elastin is formed when the lysine residues of tropoelastin are oxidized by the enzyme lysyl oxidase, yielding a reactive species, allysine, which condenses with lysine or another allysine to create lysinonorleucine or allysine aldol. These further condense to form tetrafunctional cross-linkers, desmosine or isodesmosine as shown in Figure $1.2 .{ }^{5}$ In this way, tropoelastin molecules are connected to form the mature matrix. 
Intensive cross-linking and hydrophobicity of tropoelastin contribute to the insolubility of elastin making it difficult to study. Numerous studies have been made to determine structure of the soluble precursor either by isolation or recombinant protein expression. Urry proposed a $\beta$-spiral secondary structure with Type II $\beta$-turns at PG residues in hydrophobic modules. ${ }^{6}$ Foster and coworkers ${ }^{7}$ suggested presence of $\alpha$-helical structure of the cross-linked peptide using circular dichroism experiments whereas Weiss and coworkers ${ }^{8}$ investigated peptides of cross-linking modules exhibiting $\alpha$-helical structure, albeit induced by adding trifluoroethanol (TFE). Aside from the secondary structure, the inverse temperature transition $\left(T_{c}\right)$ of tropoelastin and elastin-like proteins (ELP) have been extensively studied. At $T_{c}$, tropoelastin self-assembles which decreases the entropy

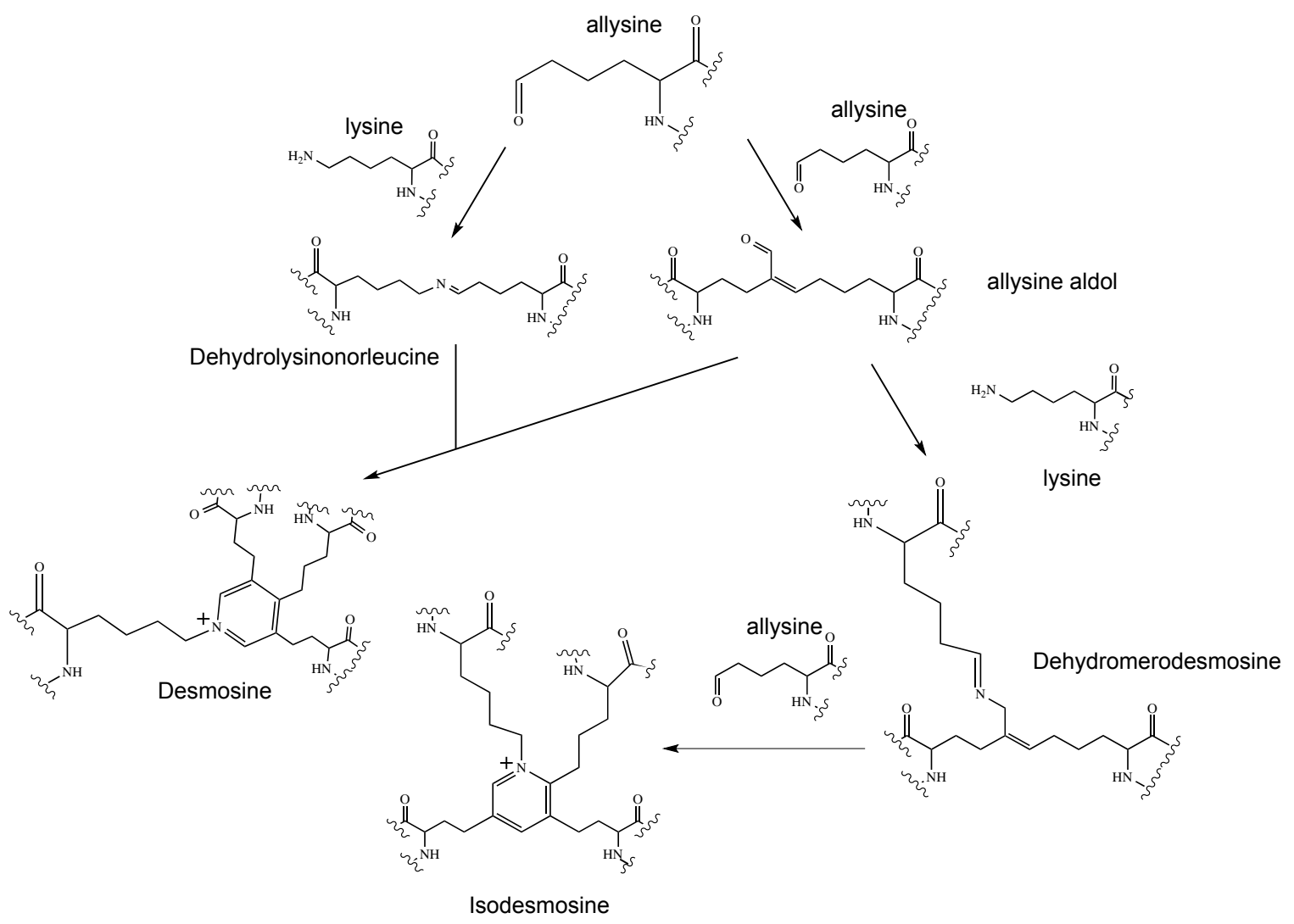

Figure 1.2 Formation of the bi-,tri-, and tetrafunctional cross-linker in elastin adapted from Vrhovski et al. ${ }^{9}$ 
of the protein. Self-assembly is driven by the hydrophobic effect. The low entropy resulting from ordering of water at hydrophobic surfaces is decreased by a loss of solvent accessible hydrophobic surface area and is favored with increased temperature because the increase in disorder of water is higher than the decrease in disorder of the protein yielding a total increase in disorder of the system. ${ }^{10}$ Hydrophobicity of the sequence, chain length and concentration affect the $\mathrm{T}_{\mathrm{c}}$ of elastin-like polypeptides.

Keeley and coworkers have pioneered a reductionist approach in recombinant expression of elastin-based proteins, minielastins. Bacterial expression was utilized to make short elastin sequences containing a small number of hydrophobic modules alternated with cross-link modules with natural sequences. These constructs have shown CD spectra comparable to native tropoelastin and were successfully cross-linked using different cross-linking agents. ${ }^{11-15}$ This approach has shown great potential in understanding the structure of tropoelastin.

\subsubsection{NMR spectroscopy}

Nuclear magnetic resonance has been used for studying elastin structure and dynamics at atomic resolution structure. Aside from X-ray crystallography and the more recent cryo-EM, NMR has been considered a reliable technique in deducing the native structure of biomolecules with molecular weights up to $\sim 50 \mathrm{kDa}$ at which point protein NMR is limited by the increased rotational correlation time resulting in high $R_{2}$ relaxation rates and broader linewidths. Structure elucidation of proteins using NMR is a routine method, particularly for folded proteins that are isotopically labeled via recombinant protein expression. 
Sequence based NMR experiments are used to assign chemical shifts for each nucleus in the protein. NMR experiments starting with ${ }^{15} \mathrm{~N}-{ }^{1} \mathrm{H}$ and ${ }^{13} \mathrm{C}-{ }^{1} \mathrm{H}$ heteronuclear single quantum coherence (HSQC) experiments gives an overview of spectral resolution in ${ }^{1} \mathrm{H}$ and ${ }^{15} \mathrm{~N} /{ }^{13} \mathrm{C}$ dimensions. These two-dimensional experiments correlates ${ }^{15} \mathrm{~N}$ or ${ }^{13} \mathrm{C}$ to the proton directly attached. Total correlation spectroscopy (TOCSY) and nuclear Overhauser effect spectroscopy (NOESY) are 2D homonuclear experiments that correlate proton pairs that are spin-coupled (TOCSY) or spatially close (NOESY). These experiments determine coupled spin-systems of protons within a residue and to identify nearby proton pairs (less than 5-6 $\AA$ apart). A problem in chemical shift degeneracy inevitably arises, especially for high molecular weight proteins and disordered proteins. In this case, spectral overlap was resolved by adding another dimension i.e. 3D experiments are utilized.

The backbone and side-chains atoms of each residue in a protein form a coupled spin system with characteristic chemical shifts and number of protons. Thus, the $3 \mathrm{D}{ }^{15} \mathrm{~N}-$ edited TOCSY-HSQC coupled with 3D ${ }^{15} \mathrm{~N}$-edited NOESY-HSQC spectrum are commonly used for amino acid assignment of ${ }^{15} \mathrm{~N}$-labelled proteins. For sidechain and backbone chemical shift assignment of proteins with known sequence, pairs of straight through and out-and-back 3D experiments are generally used. These experiments combine time domain spectroscopy and a technique that transfers spin coherence (NMR signal) between coupled spins. Importantly, the transfer can be tuned to a particular $J$ coupling. $J$-couplings in polypeptides are summarized in Figure 1.3. ${ }^{16}$ 


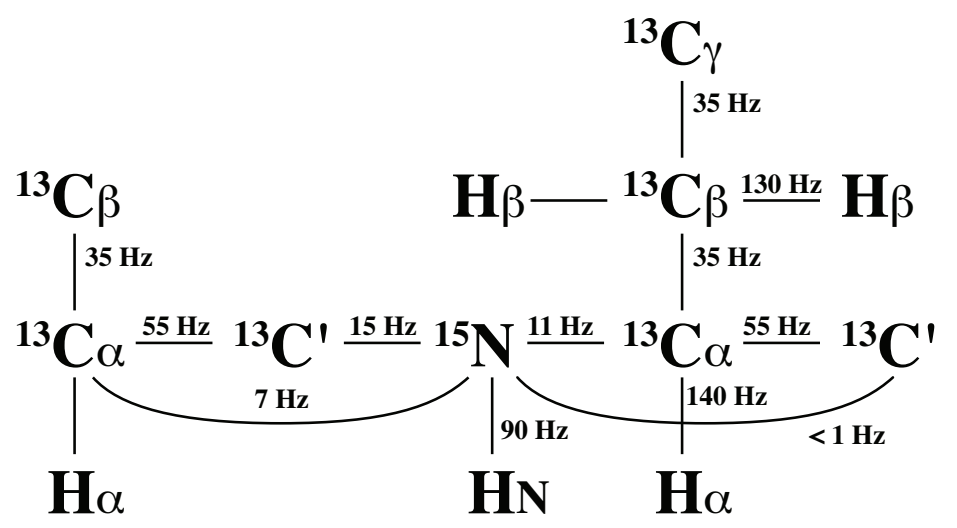

Figure 1.3. Spin system of the peptide backbone and the size of the ${ }^{1} J$ and ${ }^{2} J$ coupling constants that are used for magnetization transfer in ${ }^{13} \mathrm{C}$ and ${ }^{15} \mathrm{~N}$-labelled proteins.

A useful starting point is the $3 \mathrm{D}$ straight through experiment $\mathrm{CBCANH}$ paired with $\mathrm{CBCA}(\mathrm{CO}) \mathrm{NH}$. The coherence transfer path in the latter is from $\mathrm{C} \alpha$ and $\mathrm{C} \beta$ to $\mathrm{CO}$, then to $\mathrm{N}$ of the following residue and ultimately to the attached $\mathrm{HN}$ proton which is then

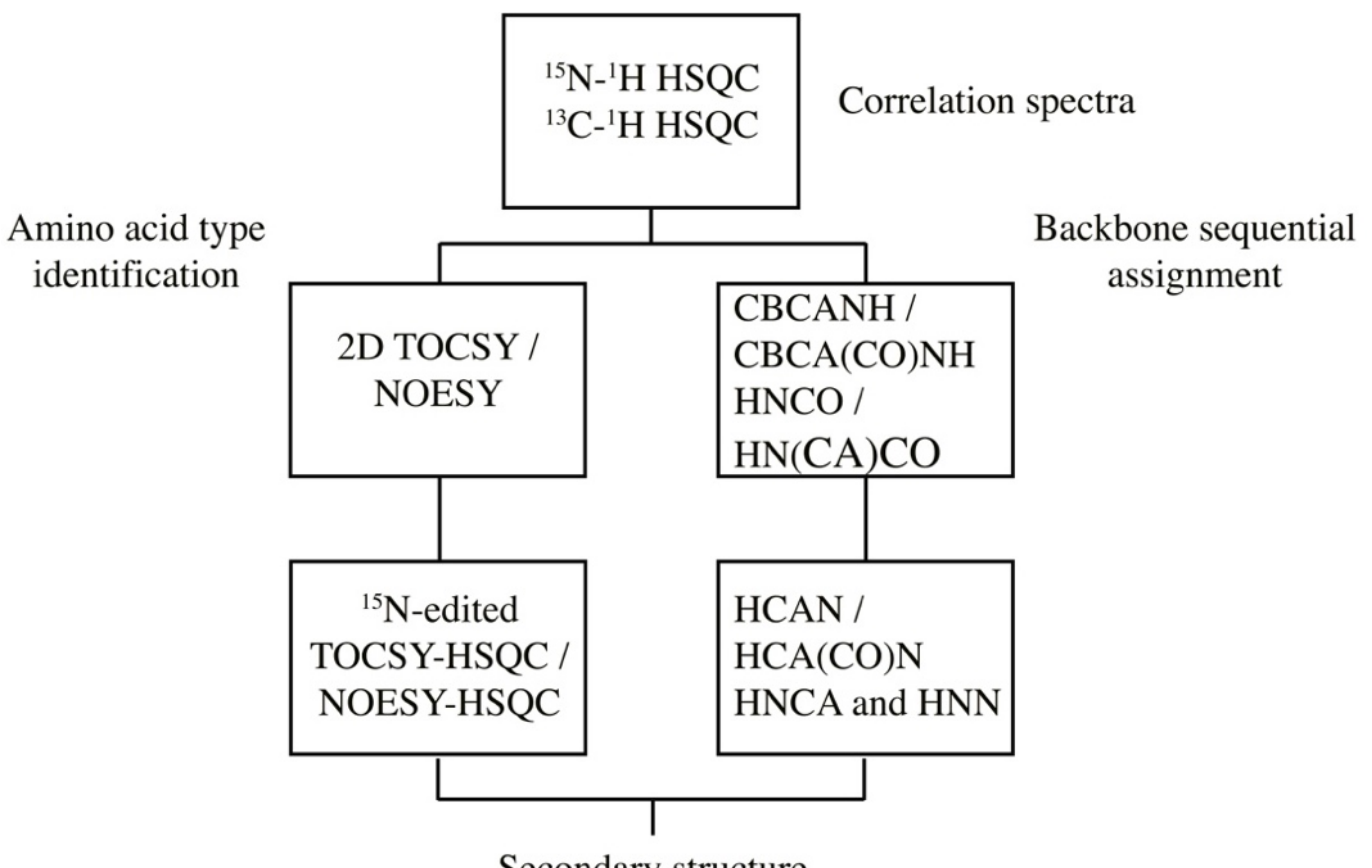

Figure 1.4. NMR strategy for structure elucidation of minielastins features $2 \mathrm{D}$ and $3 \mathrm{D}$ multinuclear experiments. 
"directly" detected as a function of the time, $t_{3}$. Incremented time delays, $t_{1}$, between the $\mathrm{C} \beta / \mathrm{C} \alpha$ to $\mathrm{N}$ transfer and $t_{2}$ between the $\mathrm{N}$ to $\mathrm{HN}$ transfer yield a $3 \mathrm{D}$ data table that, following Fourier transformation in the three time dimensions, gives the 3D spectrum with the following three correlations: a peak with $\mathrm{N}$ and $\mathrm{HN}$ shifts in the $2 \mathrm{D}{ }^{15} \mathrm{~N}-{ }^{1} \mathrm{H}$ HSQC spectrum has the $C \beta$ and $C \alpha$ shifts of the previous residue in the ${ }^{13} \mathrm{C}$ dimension. Paired with $\mathrm{CBCANH}$ these two experiments provide chemical shifts of $\mathrm{C} \alpha$ and $\mathrm{C} \beta$ correlated with the amide protons and nitrogen of residues $i$ and $i-1$. This pair of experiments are mostly effective in identifying amino acid residues with unique side chain carbon chemical shifts such as threonine, alanine, valine, and glycine. The $\mathrm{HNCO} / \mathrm{HN}(\mathrm{CA}) \mathrm{CO}$ pair of experiments provides $\mathrm{C}^{\prime}$ chemical shift values for backbone nuclei assignments. Carbonyl backbone chemical shifts are more sensitive to electronic environment and thus, can resolve overlapping peaks in ${ }^{15} \mathrm{~N}-{ }^{1} \mathrm{H}$ HSQC. Prolyl residues are not directly observed in the above-mentioned techniques, because of lack of amide proton. Hence, side chain and carbonyl nuclei are assigned using correlations from neighboring residues. $\mathrm{HCAN} / \mathrm{HCA}(\mathrm{CO}) \mathrm{N}$ experiments can also provide intra- and interresidue correlation of $\mathrm{H} \alpha, \mathrm{C} \alpha$ to $\mathrm{N}_{i}$ and $\mathrm{N}_{i+1}$ of prolyl residues. The general NMR strategy used in this study is presented in Figure 1.4.

\subsubsection{Secondary Structure}

NMR chemical shifts refers to the resonant frequencies of nuclei that vary with electronic environment. The chemical shift index (CSI) developed by Wishart et al. ${ }^{17}$ evaluates secondary structure using the difference of observed chemical shifts from accepted random coil chemical shifts. These differences are known as secondary shifts. 
Random coil shifts are obtained from simple, "unstructured" peptides. ${ }^{18}$ The CSI takes advantage of the fact that protein chemical shifts are primarily sensitive to secondary structure. ${ }^{19}$ In general, $\alpha$-helices have positive $\mathrm{C} \alpha, \mathrm{CO}$ and negative $\mathrm{H} \alpha$ and $\mathrm{C} \beta$ secondary shift values. Negative $\mathrm{C} \alpha, \mathrm{CO}$ and positive $\mathrm{H} \alpha, \mathrm{C} \beta$ shifts are obtained for $\beta$ sheets. Previous studies, albeit with limited data, suggested that the VPGV motif in elastin-like polypeptides assumes a $\beta$-spiral secondary structure where the $\mathrm{CO}$ of residues 1 and the $\mathrm{HN}$ of residue 4 form a hydrogen bond and PG residues form the bend. ${ }^{20-21}$ These results were deduced from tetra-, penta-, and hexapeptides with no cross-linking domains attached. Secondary shifts in free cross-linking domains with natural sequences were determined by Tamburro et al. and indicated $\alpha$-helical structure in trifluoroethanol (TFE). ${ }^{22}$ However, TFE is known to induce $\alpha$-helix structure. ${ }^{23-25}$

Aside from chemical shifts, NOE contacts are good indicators of secondary structure. NOE patterns for $\alpha$-helix and $\beta$-sheets are known. For helix, the presence of sequential ${ }^{1} \mathrm{H}-{ }^{1} \mathrm{H}$ distances up to $\alpha \mathrm{N}(i, i+4)$ are observed whereas $\beta$-sheets have "long range" NOE's from interstrand contacts. ${ }^{26}$ Polyproline II (PPII) helices, on the other hand, have intense $\alpha \mathrm{N}(i, i+1) / \alpha \mathrm{N}(i, i)$ as well as the absence of $\mathrm{NN}(i, i+1)$ cross peaks because of its extended structure. ${ }^{27}$ Random coil sequences exhibit a nonuniform NOE patterns but up to medium range NOEs can be observed. ${ }^{28}$

The presence of intramolecular hydrogen bonding also indicates the formation of secondary structure. Temperature coefficients of amide proton shifts are useful in predicting hydrogen bonding because they are sensitive to hydrogen bonding. Increase in temperature causes lengthening of hydrogen bonds, resulting in a decrease of the deshielding effect of the acceptor. Thus, amide protons that have intramolecular 
hydrogen bonding are less susceptible to temperature-induced chemical shift perturbation than amide protons that are hydrogen bonded with water. ${ }^{29}$ Intermolecular hydrogen bonding with water is relatively weaker. This yields a larger negative temperature coefficient compared to intramolecular hydrogen bonding.

It is important to know the factors affecting the structural and mechanical properties of elastin to fabricate elastin mimetics for a wide array of applications. Elastinlike biomaterials exhibit great potential in tissue engineering and as self-assembling materials. In this dissertation, elastin mimetics that have symmetrized hydrophobic and cross-link modules based on common motifs found in native human tropoelastin were recombinantly expressed and investigated. 


\subsection{Materials and Methods}

Minielastin constructs were recombinantly expressed in collaboration with Dr. Ronald Koder in The City College of New York.

\subsubsection{Protein Expression}

DNA minielastin with N-termini TEV protease cleavage site was synthesized by Integrated DNA technologies (Corralville, IA). Expression vector pET32a(+) (Novagen,Inc., Madison, WI) were transformed into NiCo21(DE3) competent E.coli cells. Transformed cells were initially grown in $5 \mathrm{~mL}$ LB media with carbenicillin overnight. $100 \mu \mathrm{L}$ of the LB growth was transferred to $5 \mathrm{~mL}$ M9 media overnight. (per liter: $12.8 \mathrm{~g} \mathrm{NaHPO}_{4} \bullet 7 \mathrm{H}_{2} \mathrm{O}, 3 \mathrm{~g} \mathrm{KH}_{2} \mathrm{PO}_{4}, 0.5 \mathrm{~g} \mathrm{NaCl}, 1 \mathrm{~g}{ }^{15} \mathrm{~N} \mathrm{NH}_{4} \mathrm{Cl}, 2 \mathrm{~mL} 1 \mathrm{M}$ $\mathrm{MgSO}_{4}, 20 \mathrm{~mL} \mathrm{20 \%}$ glucose and $100 \mu \mathrm{L} 1 \mathrm{M} \mathrm{CaCl}_{2}$ ). The suspension was transferred to 1 L M9 media with carbenicillin and was incubated at $37{ }^{\circ} \mathrm{C}$ until $\mathrm{OD}_{600}=0.8$, expression induced $200 \mathrm{mg}$ IPTG cold room overnight.

\subsubsection{Protein Purification}

Purification of minielastin constructs were performed as described in Greenland et $a l .{ }^{30}$ Cell pellets were obtained via centrifugation of the M9 media growth at $9000 \mathrm{rpm}$ $(11757 \times \mathrm{g})$ for 15 minutes. Wash buffer at $\mathrm{pH} 8.0$ containing $50 \mathrm{mM} \mathrm{NaH}{ }_{2} \mathrm{PO}_{4}, 300 \mathrm{mM}$ $\mathrm{NaCl}, 20 \mathrm{mM}$ imidazole, $3.1 \mathrm{mM} \mathrm{NaN}_{3}$ was used to resuspend the pellets. A pinch of protease inhibitor, Pefabloc, and $125 \mu \mathrm{L}$ of DNase solution were added to the resuspended pellets. DNase solution was prepared by dissolving $20 \mathrm{mg}$ DNase I in $10 \mathrm{~mL}$ of $20 \%$ glycerol with $75 \mathrm{mM} \mathrm{NaCl}$. The suspension was placed in tube rotator for an 
hour. Cell lysis was performed using French press. The Ni-NTA column was loaded with the lysate and the flow through was collected. The column was rinsed with wash buffer then the protein of interest was eluted using the elution buffer, $50 \mathrm{mM} \mathrm{NaH} \mathrm{PO}_{4}, 300$ $\mathrm{mM} \mathrm{NaCl}, 250 \mathrm{mM}$ imidazole and $3.1 \mathrm{mM} \mathrm{NaN}_{3} .125 \mu \mathrm{L}$ of $100 \mathrm{mM}$ EDTA, $50 \mu \mathrm{L}$ DTT and $1 \mathrm{~mL}$ TEV protease were added to the fractions which then was placed in the tube rotator for an hour. Affinity tag was removed using another round of Ni-NTA. The flow through containing the minielastin constructs were purified using $250 \times 20 \mathrm{~mm}$ Higgins Analytical PROTO $3000 \mathrm{C} 1810 \mu \mathrm{M}$ HPLC column, with a gradient of $20 \%-80 \%$ acetonitrile with $0.1 \%$ TFA over $60 \mathrm{~min}$. The purified constructs were verified using MALDI.

\subsubsection{NMR Sample Preparation}

Isotopically labelled minielastin constructs were dissolved in $110 \mu \mathrm{L} 200 \mathrm{mM}$ phosphate buffer pH $6\left(90 \% \mathrm{H}_{2} \mathrm{O}: 10 \% \mathrm{D}_{2} \mathrm{O}\right)$ with $25 \mu \mathrm{L}$ of $20 \mathrm{mM}$ EDTA, $10 \mu \mathrm{L} 1.6 \%$ $\mathrm{NaN}_{3}, 8.6 \mu \mathrm{L} 12 \mathrm{mM}$ Pefabloc and $1 \mu \mathrm{L} 100 \mathrm{mM}$ DSS as reference in $500 \mu \mathrm{L}$ solution. Total protein solution was $\sim 300 \mu \mathrm{M}$.

\subsubsection{NMR Experiments}

NMR spectra were obtained on a $700 \mathrm{MHz}$ Varian NMR spectrometer equipped with HCN cryoprobe and were processed using NMRPipe ${ }^{31}$ and NMRFAM-Sparky. ${ }^{32,33}$

${ }^{15} \mathrm{~N}-{ }^{1} \mathrm{H}$ HSQC was used to monitor sample integrity with at least 64 increments in the indirect dimension. 3D experiments, ${ }^{34} \mathrm{CBCANH}$ and $\mathrm{CBCA}(\mathrm{CO}) \mathrm{NH}$ pair of experiments combined with HCAN and $\mathrm{HCA}(\mathrm{CO}) \mathrm{N}$ were used to assign backbone and side chain 
chemical shifts. Carbonyl chemical shifts were assigned using $\mathrm{HNCO}$ and $\mathrm{HN}(\mathrm{CA}) \mathrm{CO}$ pair of experiments. The HNN experiment were used to confirm assignment in the crosslink modules.

NOEs were measured using ${ }^{15} \mathrm{~N}$-edited NOESY-HSQC spectra with $100 \mathrm{~ms}$ or 250 ms mix times paired with ${ }^{15} \mathrm{~N}$-edited TOCSY-HSQC spectrum with $70 \mathrm{~ms}$ mix times to assign intraresidue and sequential NOEs. All the pulse sequence used were built-in Varian Inova BioPack. 


\subsection{Results and Discussion}

\subsubsection{Chemical Shift Assignments}

The hydrophobic module 24', is a 42-residue construct containing seven sixresidue repeats with the sequence APGVGV. 24' is derived from the sequence coded by exon 24 of tropoelastin. A narrow range of peaks in the amide proton $(\mathrm{HN})$ dimension was observed in the ${ }^{15} \mathrm{~N}-{ }^{1} \mathrm{H}$ HSQC spectrum which is a characteristic of an intrinsically disordered protein. ${ }^{35}$ The ${ }^{15} \mathrm{~N}-{ }^{1} \mathrm{H}$ HSQC spectrum shown in Figure 1.5a contains five major, high intensity peaks and four minor peaks representing the 42-residue peptide. This shows chemical shift averaging and that the APGVGV-repeat peptide is flexible and primarily unstructured. The ${ }^{13} \mathrm{C}-{ }^{1} \mathrm{H}$ HSQC spectrum, Figure $1.5 \mathrm{~b}$, shows anti-phase peaks of $\mathrm{CH}_{2}$ carbons. Unique sidechain carbon chemical shifts are helpful in assigning resonances in ${ }^{13} \mathrm{C}-{ }^{1} \mathrm{H}$ HSQC. Moreover, the ${ }^{13} \mathrm{C}-{ }^{1} \mathrm{H}$ HSQC is consistent with the ${ }^{15} \mathrm{~N}-{ }^{1} \mathrm{H}$ HSQC spectrum and that carbon shifts are more sensitive to secondary structure than amide proton shifts. The fingerprint region of TOCSY shows five spin systems, Figure 1.5c. Note that the alanine and glycine are degenerate in $\mathrm{HN}$ dimension, however, glycine has a distinct upfield $\mathrm{H} \alpha$ at $\sim 3.9 \mathrm{ppm}$. Also, each valine has unique $\mathrm{H} \beta$ and $\mathrm{H} \gamma$ chemical shifts. Sidechain resonances of P2 were assigned using the TOCSY aliphatic region, Figure 1.5d, whereas the glycines, G3 and G5, and the valines, V4 and V6, were differentiated using the NOESY experiment. The TOCSY spectrum was superimposed with the NOESY spectrum, Figure 1.6, to differentiate the inter-residue NOE versus the intra-residue NOE. The fingerprint region shows correlation between the side chain of residue $i-1$ to amide proton of residue $i$. Glycine $(\sim 8.4 \mathrm{ppm})$ shows NOE peaks from the proline side chain, previously assigned using TOCSY. This glycine is assigned as G3 

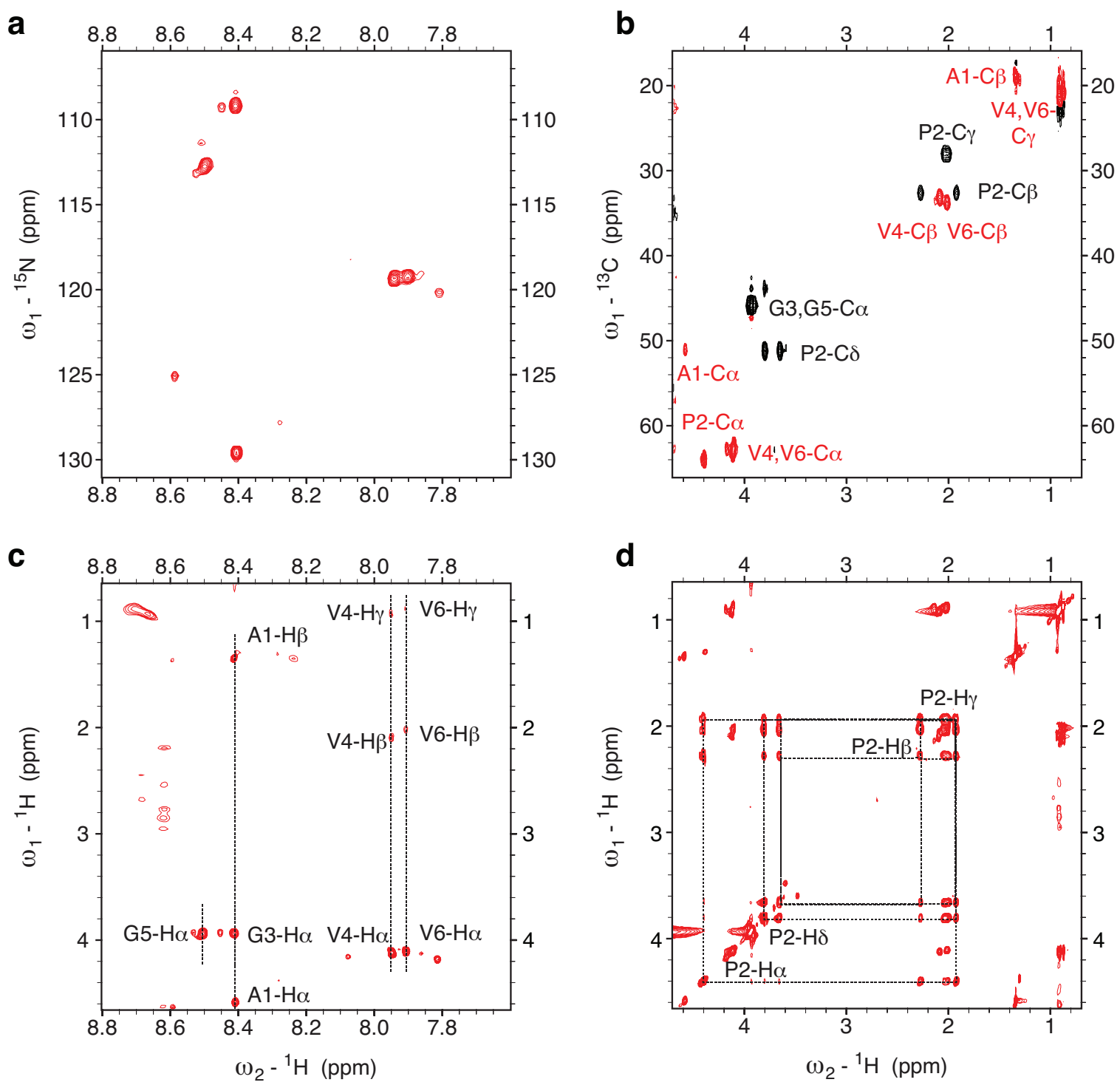

Figure 1.5. Two-dimensional NMR spectra of 24 . (a) The ${ }^{15} \mathrm{~N}-\mathrm{H}$ HSQC has five major peaks correspond to the 6-residue repeat. Proline is not seen due to the absence of an amide proton. (b) The ${ }^{13} \mathrm{C}-\mathrm{H}$ HSQC spectrum shows the characteristic carbon chemical shifts for side chain resonances. (c) The TOCSY fingerprint region shows the spin system for each peak in the ${ }^{15} \mathrm{~N}-{ }^{1} \mathrm{H}$ HSQC utilized in identifying amino acids. (d) Aliphatic region of TOCSY spectrum used to assign proton chemical shifts of proline side chain. 
and the remaining peak with glycine spin system is assigned to G5. V4 was assigned using the NOE correlation with $\mathrm{G} 5$, as $\alpha \mathrm{N}_{i-1}-\mathrm{HN}_{i}$ shows sequential cross-peaks. Natural abundance $24^{\prime}$ was used in this experiment, thus, $\mathrm{C}^{\prime}$ chemical shifts were not obtained. Incomplete resonance assignments reported in Table 1.1.

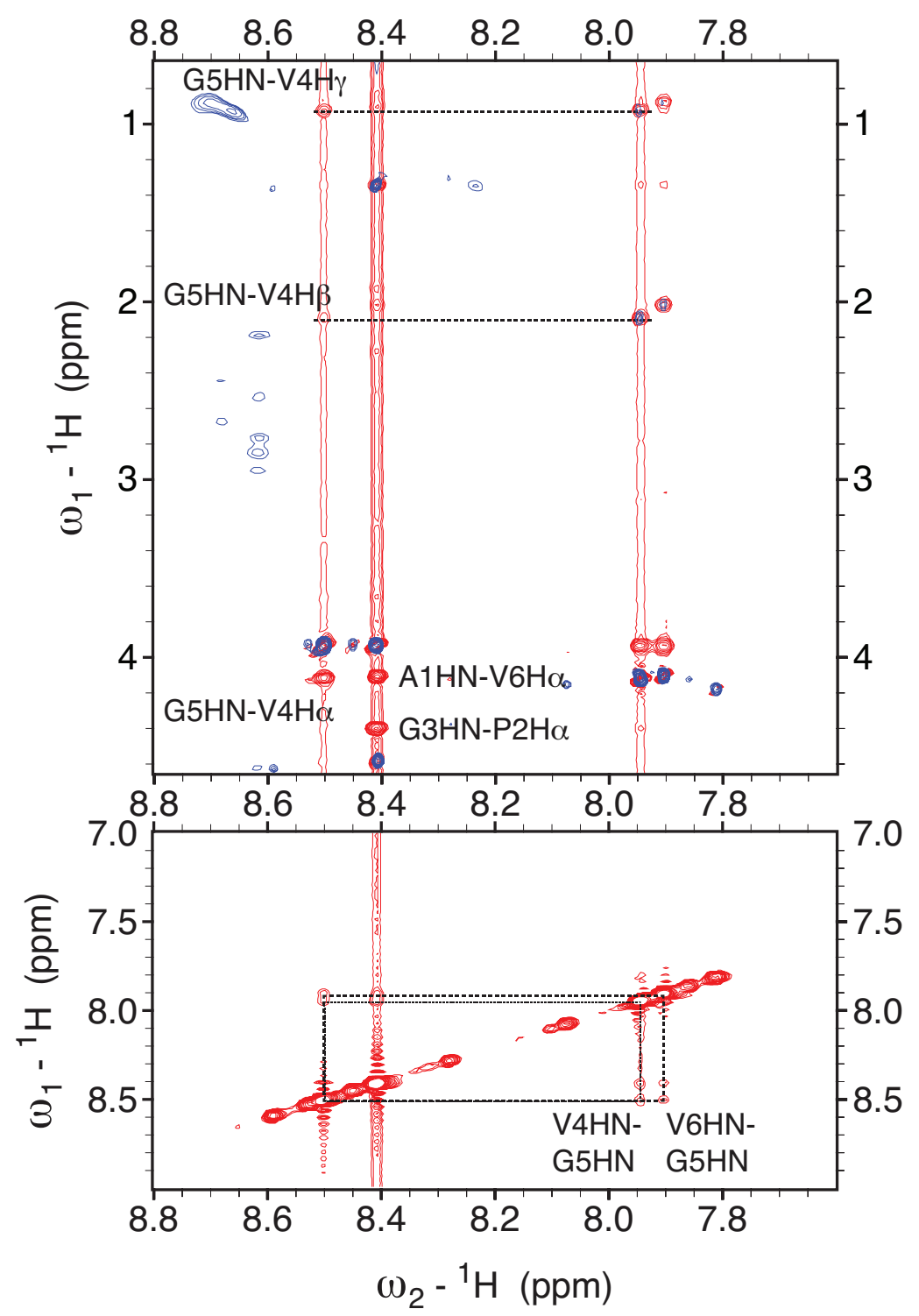

Figure 1.6 NOESY (red) overlaid with TOCSY (blue) fingerprint (top) and amide (bottom) region to differentiate peaks from neighboring residues. 


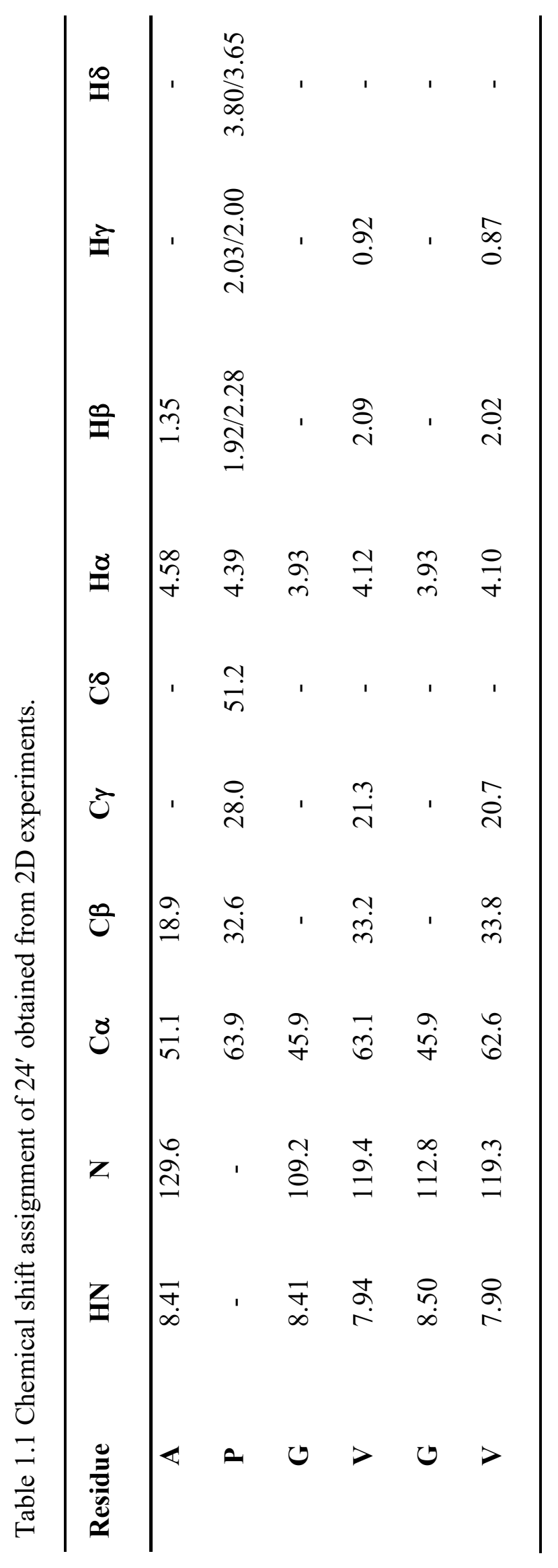


In this study, two types of cross-link sequence, $\mathrm{X}$ and $\mathrm{x}^{\prime}$ were utilized. The longer sequence, $\mathrm{X}$, is the wild type tropoelastin sequence coded by exon 21 and 23 . Both contain a single $\mathrm{A}_{5} \mathrm{KA}_{2 / 3} \mathrm{~K}$ motif that is found in all of elastin's KA cross-link modules. Similarly, $\mathrm{x}^{\prime}$ is an 11-residue sequence that contains one of the $\mathrm{A}_{5} \mathrm{KA}_{2} \mathrm{~K}$ motifs coded by exons 21 and 23. The use of this simpler, shorter cross-link sequence makes the NMR spectrum less complicated, although, the challenge of assigning sequential alanine resonances remains. Since X with 33 residues consists primarily of alanine, it has a more complicated spectrum compared to $x^{\prime}$, Figure $1.7 \mathrm{a}$ and $1.8 \mathrm{a}$. The $\mathrm{A}_{5} \mathrm{KA}_{2 / 3} \mathrm{~K}$ motifs of $\mathrm{X}$ are separated by the sequence GVGTP which introduces kinks and/or bends in the crosslink module. Even with TOCSY and NOESY, because of multiple overlaps of alanine signals, complete chemical shift assignments for each resonance were not possible, especially in NMR experiments using natural abundance ${ }^{13} \mathrm{C}$ and ${ }^{15} \mathrm{~N}$. Nonetheless, TOCSY was used to identify residues such as glycine, threonine, valine and alanine, Figure $1.7 \mathrm{c}$. Glycines are generally easy to assign because of the distinct upfield ${ }^{15} \mathrm{~N}$ chemical shift. G14 and G16 were assigned using sidechain peaks from V15 and T17 respectively, whereas A19 shows NOE cross-peaks from sidechains of P18. F32 and Y13. Both have downfield $H \beta$ chemical shifts, $\sim 3 \mathrm{ppm}$, caused by the deshielding from the adjacent phenyl ring. The Y13 H $\alpha$, on the other hand, shows correlation with G14 that differentiated Y13 from F32.

The natural abundance ${ }^{15} \mathrm{~N}-{ }^{1} \mathrm{H}$ HSQC spectrum of $\mathrm{x}^{\prime}$, Figure 1.8, has 10 peaks from 11 residues. Amide protons from the N-terminus are typically not observed in the ${ }^{15} \mathrm{~N}-{ }^{1} \mathrm{H}$ HSQC spectrum. Most peaks are resolved in the ${ }^{15} \mathrm{~N}$ dimension but not well dispersed in the proton dimension as shown in Figure $1.8 \mathrm{a} .{ }^{13} \mathrm{C}-{ }^{-1} \mathrm{H}$ HSQC allowed 
assignment of side chain resonances however, the carbon and proton side chains resonances of alanines are very similar. ${ }^{13} \mathrm{C}-{ }^{1} \mathrm{H}$ HSQC paired with TOCSY is useful in identifying amino acids but even with 2D NOESY experiment and good resolution in ${ }^{15} \mathrm{~N}$ dimension, it was challenging to provide sequential assignment because of resonance degeneracy. The full chemical shift assignment was completed using three dimensional experiments.
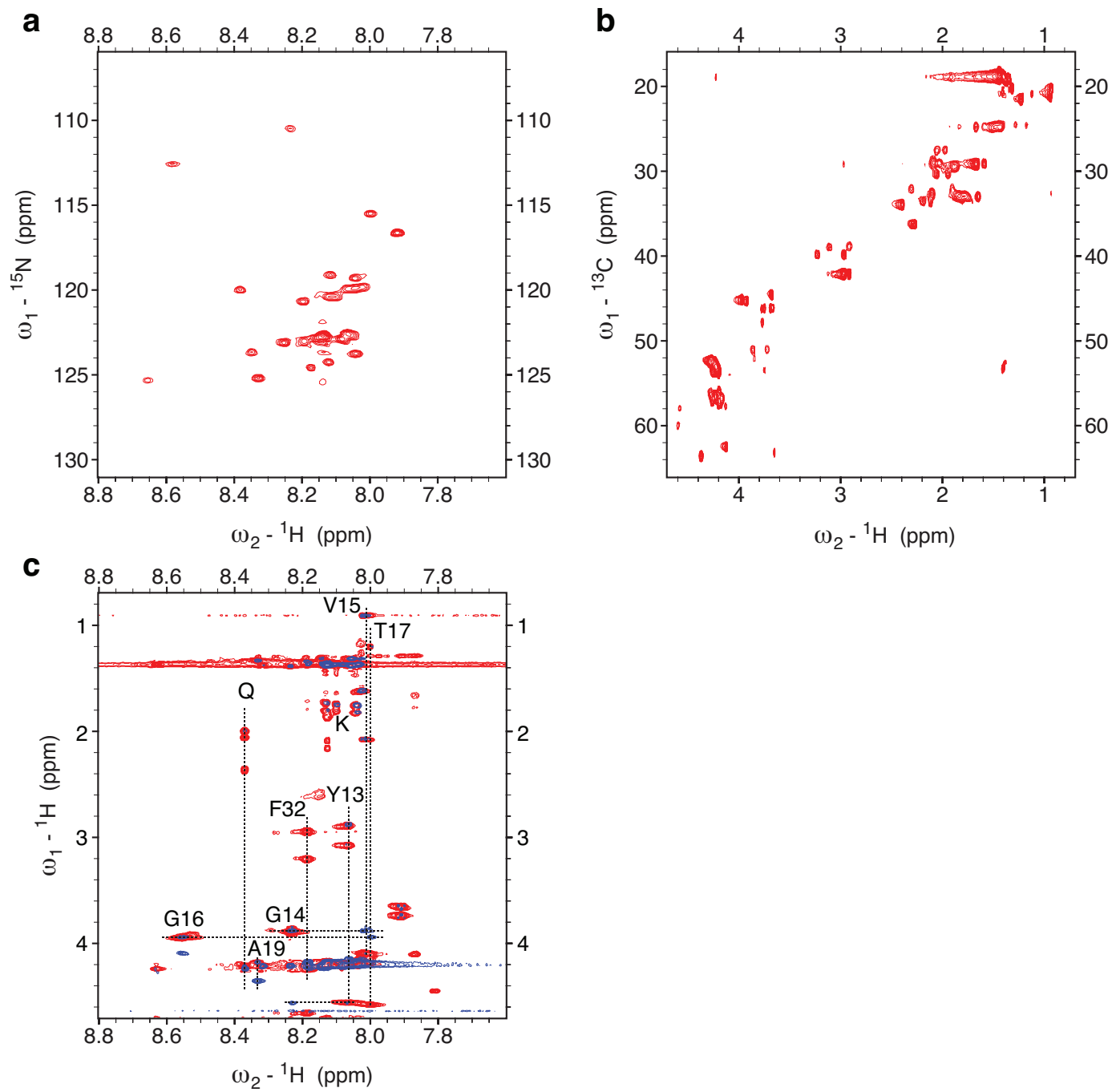

Figure 1.7 Two-dimensional of the unlabeled X cross-link module. (a) The ${ }^{15} \mathrm{~N}-{ }^{1} \mathrm{H}$ HSQC shows poor resolution of alanine nitrogen chemical shifts $\sim 122 \mathrm{ppm}$. (b) The ${ }^{13} \mathrm{C}-{ }^{1} \mathrm{H}$ HSQC and (c) TOCSY (red) overlaid with NOESY (blue). 

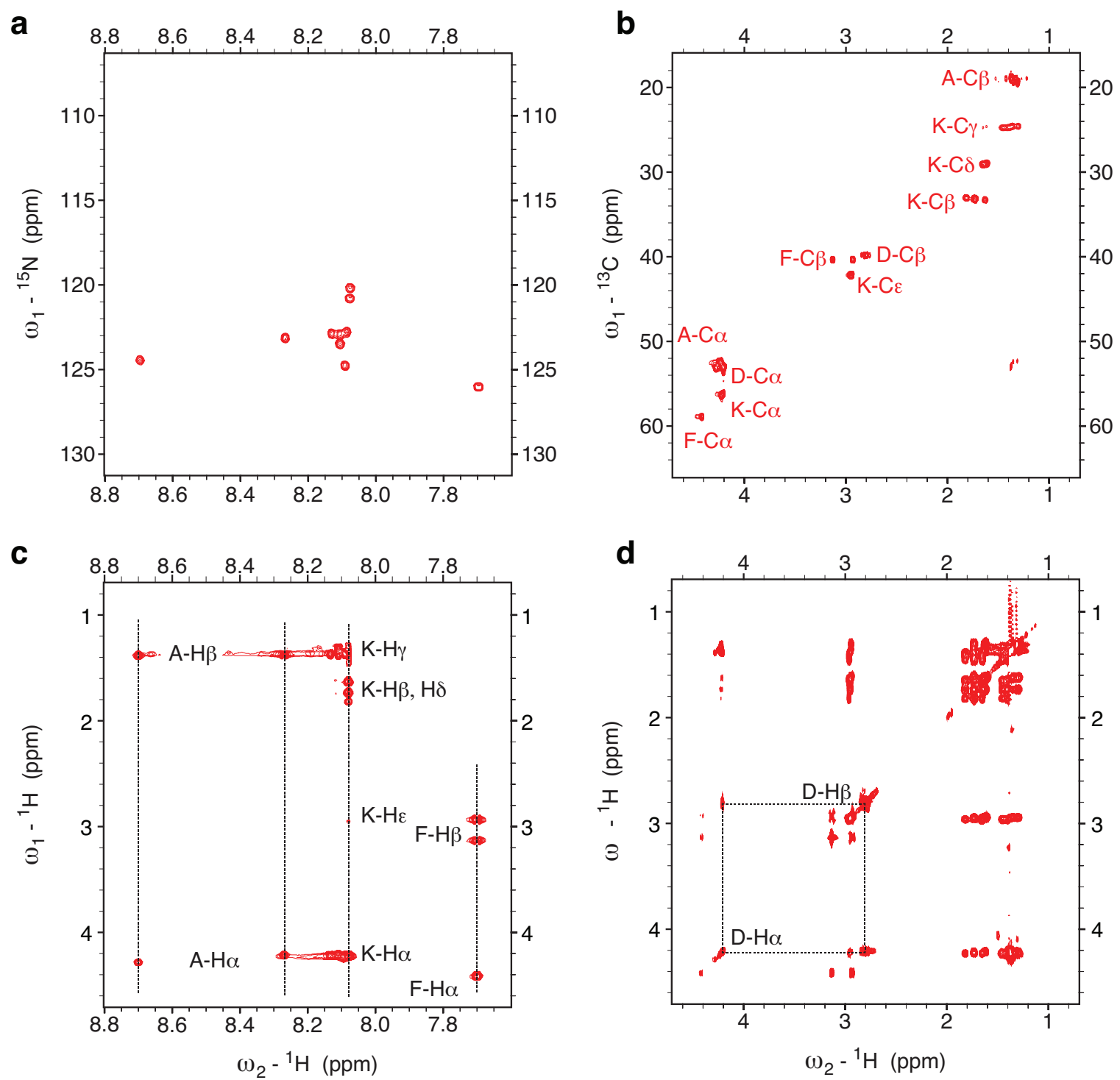

Figure 1.8 Two-dimensional spectra of unlabeled $\mathrm{x}^{\prime}$. (a) ${ }^{15} \mathrm{~N}-{ }^{1} \mathrm{H}$ HSQC of $\mathrm{x}^{\prime}$ shows ten peaks corresponding to 11 residues (N-terminus not seen), mostly resolved in the ${ }^{15} \mathrm{~N}$ and ${ }^{1} \mathrm{H}$ dimension. (b) ${ }^{13} \mathrm{C}-{ }^{1} \mathrm{H}$ HSQC assignments based on average carbon and proton chemical shifts of protein side chains. High degeneracy observed for alanine side chain resonances. (c) TOCSY fingerprint region shows spin system for different type of residue. Chemical shifts for alanines are very similar which makes resonance assignment challenging. (d) TOCSY aliphatic region shows presence of aspartate, N-terminus, side chain proton chemical shifts. 


\subsubsection{Minielastin Constructs}

Tropoelastin is a hydrophobic protein, precursor to elastin. It has alternating hydrophobic and cross-link modules. The goal is to understand how the structure of tropoelastin contributes to the elastic property of elastin. Keeley and coworkers utilized hydrophobic and cross-link sequences from the wild type exons of tropoelastin that mimics the alternating hydrophobic and cross-link module as in tropoelastin.

In the minielastin constructs prepared and studied here, the hydrophobic sequences contain only the predominant 6-residue repeats coded by exons 20 and 24 and named $20^{\prime}$ and $24^{\prime} .20^{\prime}$ has five VPGVGG repeats and $24^{\prime}$ has APGVGV repeated seven times. As previously discussed, two variations of cross-link sequence were used, $\mathrm{X}$ and $\mathrm{x}^{\prime}$. The description of each construct is summarized in the Table 1.2. Note that each construct has a tryptophan in $\mathrm{N}$ and $\mathrm{C}$-termini to provide absorbance needed for detection in HPLC.

Table 1.2 Names, number of residues, molecular weights and sequences of the minielastin constructs.

\begin{tabular}{cccc}
\hline Name & \#aa's & MW (Da) & Sequence \\
\hline $\mathbf{2 4 \mathbf { x } ^ { \prime }}$ & 203 & 16,895 & $24^{\prime}-\mathrm{x}^{\prime}-24^{\prime}-\mathrm{x}^{\prime}-24^{\prime}-\mathrm{x}^{\prime}-24^{\prime}$ \\
$\mathbf{2 0 \mathbf { x } ^ { \prime }}$ & 138 & 11,483 & $20^{\prime}-\mathrm{x}^{\prime}-24^{\prime}-\mathrm{x}^{\prime}-24^{\prime}$ \\
$\mathbf{2 0 2 4 2 4}$ & 182 & 15,364 & $20^{\prime}-\mathrm{X}-24^{\prime}-\mathrm{X}-24^{\prime}$ \\
$\mathbf{2 0 2 0}$ & 254 & 21,060 & $20^{\prime} 20^{\prime}-\mathrm{X}-24^{\prime}-\mathrm{X}-24^{\prime} 24^{\prime}$ \\
\hline $\begin{array}{l}\text { 24:(APGVGV) } \\
\text { X: } 20^{\prime}:(\text { VAPGVGG) }\end{array}$ & & \\
\end{tabular}


The simplest construct, $24 \mathrm{x}^{\prime}$, has three short cross-link sequences, $\mathrm{x}^{\prime}$ flanked by four $24^{\prime}$ hydrophobic modules. Figure 1.9 shows the overlay of ${ }^{15} \mathrm{~N}-{ }^{1} \mathrm{H}$ HSQC spectra from the minielastin, $24 x^{\prime}$ (red) with spectra of the constituent hydrophobic, 24' (black) and crosslink, $\mathrm{x}^{\prime}$ (blue), modules. Chemical shifts of $24^{\prime}$ residues are unchanged when flanked by $x^{\prime}$ in $24 x^{\prime}$, however, those of $x^{\prime}$ in $24 x^{\prime}$ are changed drastically compared to the free peptide. This suggests that the structure of the hydrophobic module is independent of the cross-linking module. In addition, the structure of the cross-linking module changes when flanked with hydrophobic module. The hydrophobic residues were assigned using the previous assignment for $24^{\prime}$. Note that the amide proton chemical shifts of $24 \mathrm{x}^{\prime}$ lies within a $1 \mathrm{ppm}$ range which suggests disorder. ${ }^{15} \mathrm{~N}-{ }^{1} \mathrm{H}$ HSQC of $24 \mathrm{x}^{\prime}$ showed good resolution at ${ }^{15} \mathrm{~N}$ dimension which helped in assigning carbon resonances in CBCANH/CBCACONH experiments.

Each peak in the ${ }^{15} \mathrm{~N}-{ }^{1} \mathrm{H}$ HSQC corresponds to a $\mathrm{C} \alpha$ and $\mathrm{C} \beta$ pair that belongs to residue $i-1$ for CBCACONH whereas CBCANH contains peaks for both $i$ and $i-1$. Figure 1.10 shows the CBCACONH/CBCANH spectra obtained for the hydrophobic and crosslink module of $24 x^{\prime}$. CBCANH shows anti-phase peaks for $\mathrm{C} \alpha$ (green) and $\mathrm{C} \beta$ (red) which helps distinguish $C \alpha$ from $C \beta$ aside from more downfield shifts of $C \alpha$. Peaks from CBCACONH spectrum shown in blue to help visualize peaks belonging to $i-1$ residue. The hydrophobic module assignment was verified as shown in the top panel of Figure 1.10. G3 shows correlation with $\mathrm{P} 2$ (C $\alpha \sim 63 \mathrm{ppm}, \mathrm{C} \beta \sim 32 \mathrm{ppm}), \mathrm{G} 5$ with V4 which has more downfield $\mathrm{C} \alpha$ chemical shift than V6. In this experiment, the lysine residues buried in the high intensity valine peaks were observed. More upfield $\mathrm{C} \alpha$ chemical shifts of lysines distinguish them from valines whereas $C \beta$ chemical shifts are very similar. 


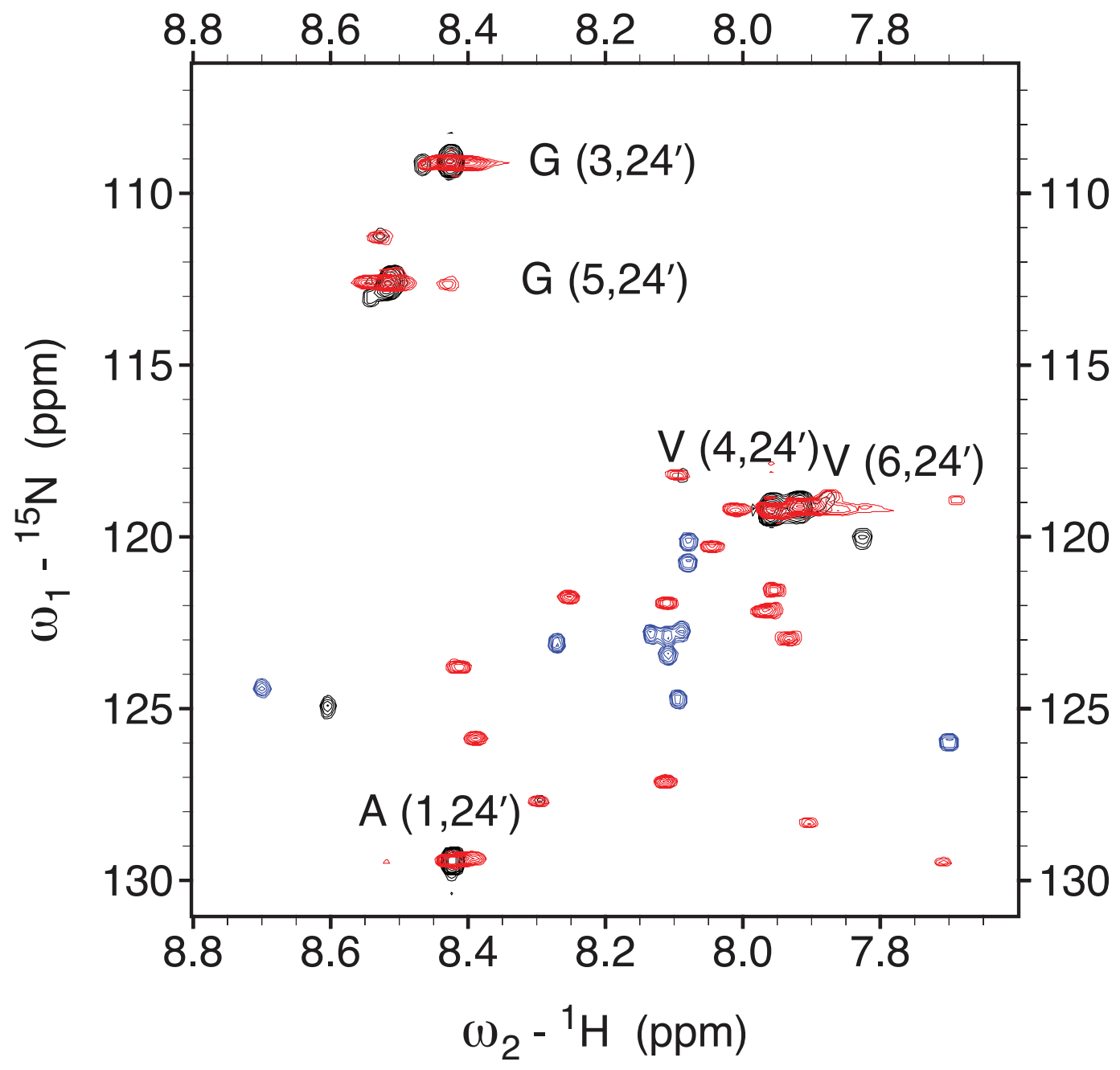

Figure $1.9^{15} \mathrm{~N}-{ }^{1} \mathrm{H}$ HSQC of $24 \mathrm{x}^{\prime}$ (red), 24' (black) and $\mathrm{x}^{\prime}$ (blue). All proton shifts are within a $1 \mathrm{ppm}$ range. Assignments were taken from $24^{\prime}$ as it matches the peaks in $24 \mathrm{x}^{\prime}$ construct. 

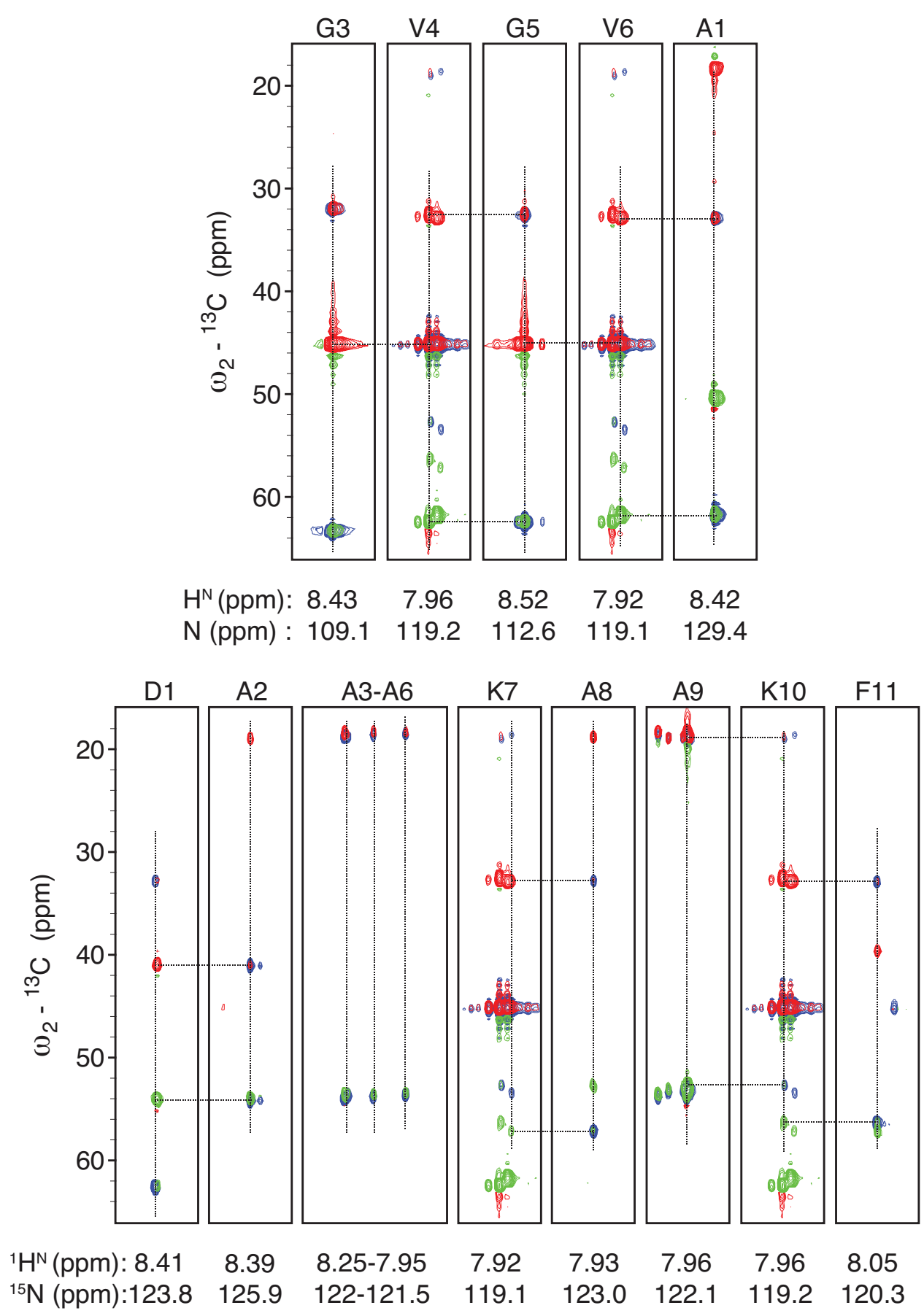

Figure 1.10 Strips from the CBCACONH spectrum (blue) overlaid with those from $\mathrm{CBCANH}$. C $\alpha$ signals are shown in green and $\mathrm{C} \beta$ are in red except for glycine residues. The CBCACONH spectra show side-chain peaks belonging to i-1 residues for both hydrophobic (top) and cross-link (bottom) residues. Alanine side chains in the cross-link module have very similar chemical shifts. 
V4 and V6 panels show K7 and K10 lysine residues of the cross-link module. Lysines appear as lower intensity peaks at ${ }^{15} \mathrm{~N}$ and ${ }^{1} \mathrm{H}$ chemical shifts overlapping with valine residues. Therefore, these peaks were not resolved in ${ }^{15} \mathrm{~N}-{ }^{1} \mathrm{H}$ HSQC spectrum. Moreover, alanine carbon side chains have very similar chemical shifts. D1 of the cross-link module had a unique downfield $\mathrm{C} \beta$ chemical shift caused by the adjacent carbonyl. A2 Peaks belonging to $\mathrm{A} 3$ to $\mathrm{A} 6$ and $\mathrm{A} 9$ were more challenging to assign because these alanines are preceded with alanines. The two lysines have unique carbon chemical shifts. These were used to distinguish $\mathrm{K} 7$ from K10. K10 is followed by F11 which was assigned due to its distinct side chain carbon and proton chemical shifts. Also, identifying A8 verifies this assignment. Note that $\mathrm{A} 8$ is the only alanine residue preceded by lysine. Nonetheless, to assign the cross-link residues in the ${ }^{15} \mathrm{~N}-{ }^{1} \mathrm{H}$ HSQC, the $\mathrm{HNCO} / \mathrm{HNCACO}$ pair of experiments was utilized. As shown in Figure 1.11, backbone $\mathrm{C}^{\prime}$ for the alanines are mostly similar but the higher resolution of the $3 \mathrm{D}$ experiment

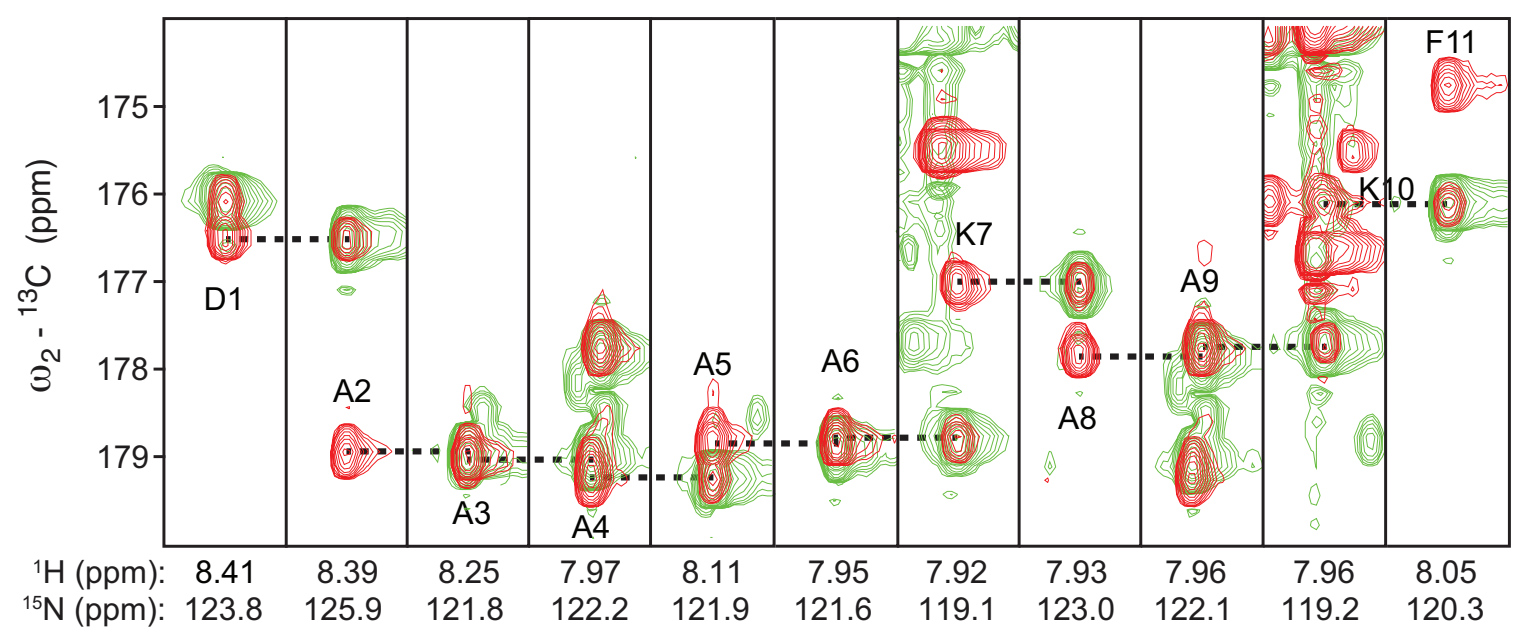

Figure 1.11 Sequential assignment of the cross-link module using strips from the HNCO (green) and HNCACO (red) spectra. 
resolves each residue in the cross-link module, including the lysine peaks. The $\mathrm{C}^{\prime}$ chemical shifts of lysines are much different than the valines unlike the side chain carbons as previously shown. The summary of the backbone and side chain resonances of $24 \mathrm{x}^{\prime}$ is found in Appendix I-A.

A variation of $24 x^{\prime}, 20 x^{\prime}$, has shown similar peaks as $24 x^{\prime}$. As indicated in Table $1.2,24 \mathrm{x}^{\prime}$ and $20 \mathrm{x}^{\prime}$ contains the same cross-link module but has a $20^{\prime}$ sequence as the $\mathrm{N}$ terminus hydrophobic module. $20^{\prime}$ has more glycine residues and is expected to be more flexible and unstructured. Shown in Figure 1.12 are the superimposed ${ }^{15} \mathrm{~N}-{ }^{1} \mathrm{H}$ HSQC spectra of $24 x^{\prime}$ (red) and $20 x^{\prime}$ (black). The overlapped area is attributed to the $24^{\prime}-x^{\prime}-24^{\prime}$ part of the $20 x^{\prime}$ sequence. The hydrophobic module $20^{\prime}$ contributions to the overall spectrum are isolated due to their greater peak intensity than the cross-link peaks. Interestingly, the cross-link residues after $20^{\prime}$ have different chemical shifts compared to the cross-link residues after $24^{\prime}$ indicating the different environment provided to crosslink modules by different hydrophobic modules. Assignments from $24 \mathrm{x}^{\prime}$ could be used to assign the majority of peaks and additional analysis was done for $20 \mathrm{x}^{\prime} .{ }^{15} \mathrm{~N}$-labeled $20 \mathrm{x}^{\prime}$, because it is only ${ }^{15} \mathrm{~N}$ enhanced, makes it more difficult to assign carbon chemical shifts. Nonetheless, the 3D experiment such as the TOCSY-HSQC and NOESY-HSQC pair was utilized to accomplish sequence assignments as shown in Figure 1.13. The resolution of some alanine peaks in the proton dimension helped in assigning alanine peaks of crosslink modules using the NOESY $-{ }^{15} \mathrm{~N}-{ }^{1} \mathrm{H}$ HSQC.

Similarly, the assignment of lysine peaks was challenging because of overlap with intense valine peaks. Carbon chemical shifts were assigned using TOCSY-HSQC with ${ }^{13} \mathrm{C}-{ }^{1} \mathrm{H}$ HSQC. This provided a lower resolution compared to doing a 3D experiment with 


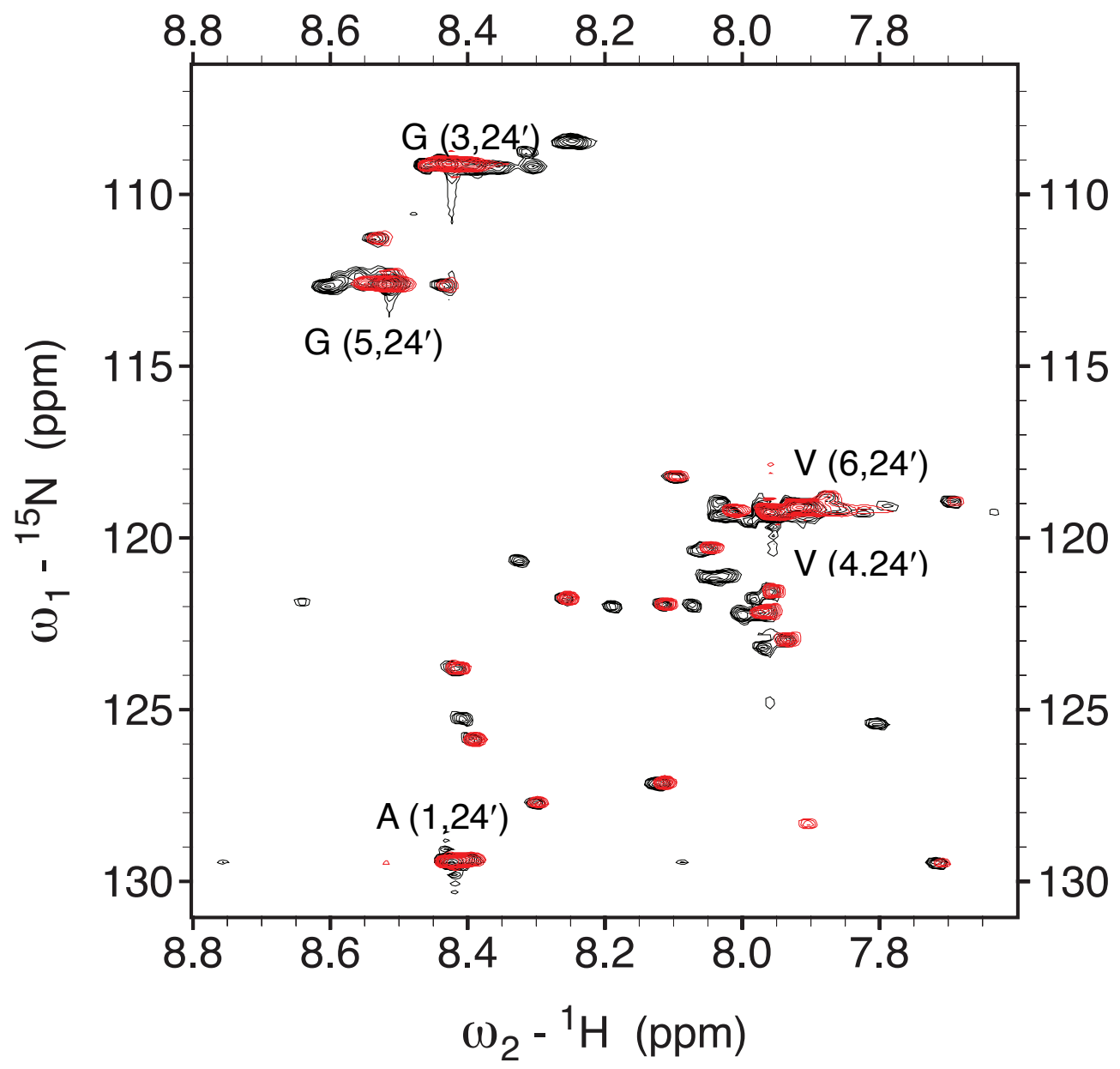

Figure 1.12 Overlaid ${ }^{15} \mathrm{~N}-{ }^{1} \mathrm{H}$ HSQC of $20 \mathrm{x}^{\prime}$ (black) and $24 \mathrm{x}^{\prime}$ (red). Additional peaks coming from the cross-link residues after $20^{\prime}$ were differentiated as these peaks do not overlap. 


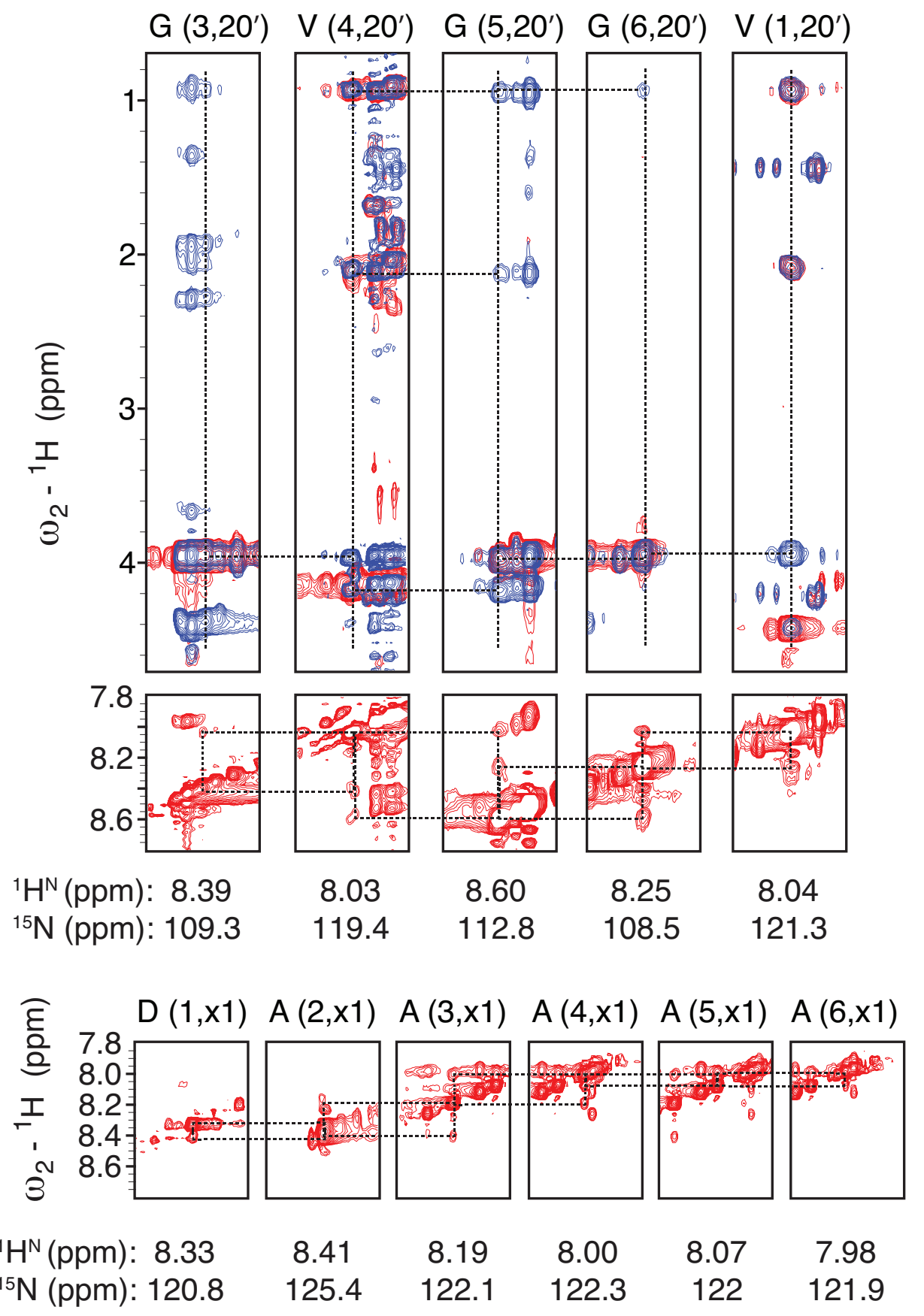

Figure $1.13{ }^{15} \mathrm{~N}$-edited TOCSY-HSQC (red) paired with ${ }^{15} \mathrm{~N}$-edited NOESY-HSQC (blue) of ${ }^{15} \mathrm{~N}$-labeled $20 \mathrm{x}^{\prime}$, (top) fingerprint and amide region of the hydrophobic module $20^{\prime}$ and (bottom) amide region of the cross-link module. 


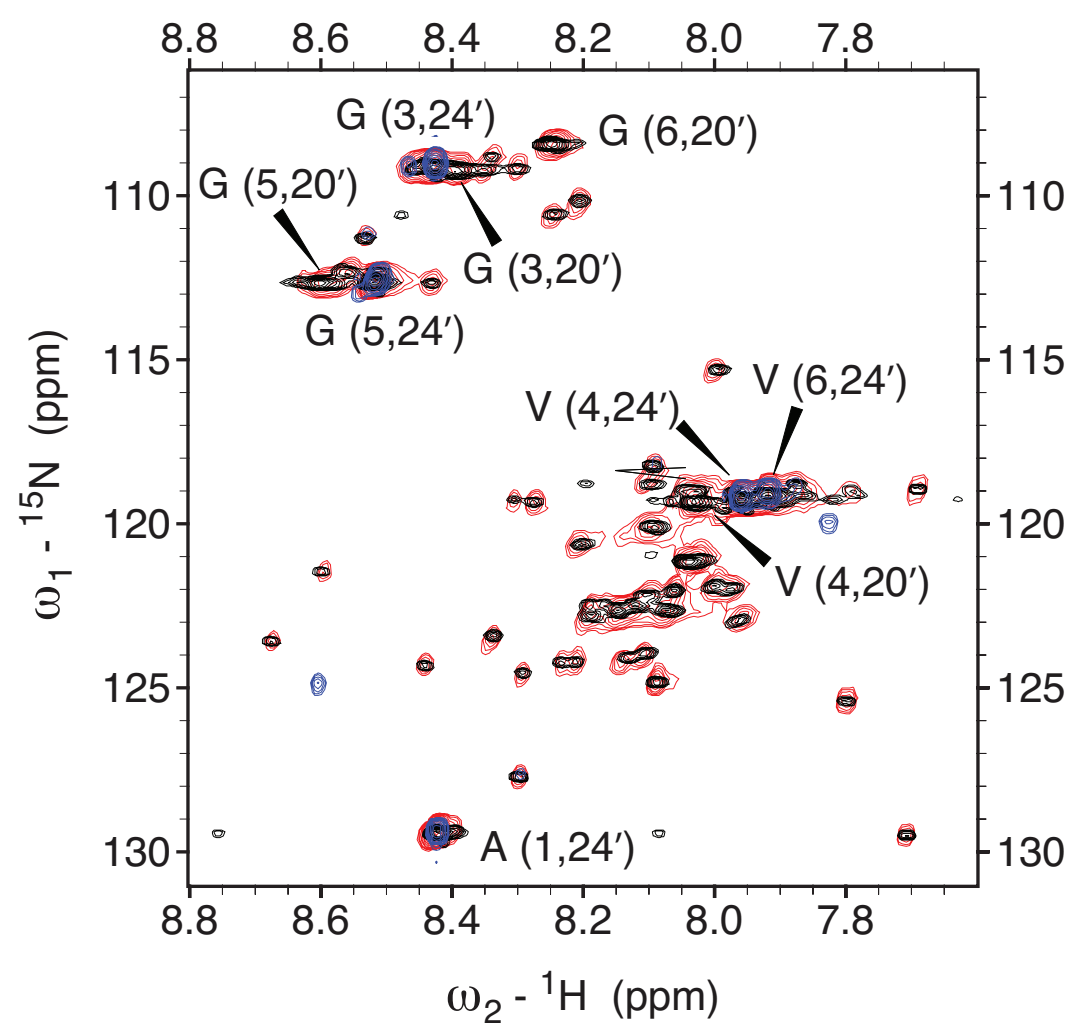

Figure 1.14 Overlaid spectra of the ${ }^{15} \mathrm{~N}^{-1} \mathrm{H}$ HSQC of 202424 (red), 2020 (black) and 24' (blue).

doubly labeled protein. Nonetheless, most peaks were assigned previously from $24 \mathrm{x}^{\prime}$. Shown in Appendix I-B is the chemical shift assignment for $20 \mathrm{x}^{\prime}$.

Figure 1.14 shows how the chemical shifts of the hydrophobic modules are conserved. As shown in this chapter, chemical shifts of the hydrophobic modules with or without cross-link modules were unchanged and only the chemical shifts of few amino acids adjacent the cross-link modules had changed. Not so surprisingly, the spectrum of 2020 superimposes with 202424 as the additional hydrophobic residues i.e. (VPGVGG)5 and $(\mathrm{APGVGV})_{7}$, have degenerate chemical shifts thus, additional residue does not increase the number of peaks shown in the ${ }^{15} \mathrm{~N}-{ }^{1} \mathrm{H}$ HSQC, instead, increases the intensity 
of the hydrophobic residue peaks. Chemical shift assignment for 202424 and 2020 were accomplished using the same approach as $24 x^{\prime}$. Analogous to previous constructs, chemical shift assignments for the alanine-rich cross-link module and degenerate peaks in ${ }^{15} \mathrm{~N}-{ }^{1} \mathrm{H}$ HSQC will only be possible using multidimensional NMR experiments therefore, double-labeled minielastin constructs were expressed.

Few residues of the two cross-link modules are expected to have different chemical shifts as previously observed with $20 \mathrm{x}^{\prime}$. E $(1, \mathrm{X} 1)$ was differentiated with $\mathrm{E}$ $(1, \mathrm{X} 2)$ using $\mathrm{CBCANH} / \mathrm{CBCACONH}$ experiment. $\mathrm{E}(1, \mathrm{X} 1)$ was preceded with glycine $(\mathrm{C} \alpha \sim 45 \mathrm{ppm})$ whereas $\mathrm{E}(1, \mathrm{X} 1)$ was preceded with valine $(\mathrm{C} \alpha \sim 63 \mathrm{ppm}, \mathrm{C} \beta \sim 32 \mathrm{ppm})$ as shown in Figure 1.15. Moreover, the connectivity of EAQA of X1 and X2 were verified by $\mathrm{HNCO} / \mathrm{HNCACO}$. Q3 and $\mathrm{A} 4$ of $\mathrm{X} 2$ were more downfield than that of $\mathrm{X} 1$. Same strategy was applied with the GVGTP sequence found at the middle of the crosslink module. G14 was assigned using NOESY-HSQC. V15 was challenging to assign because of poor resolution and intense signal coming from the valines of the hydrophobic modules. Nonetheless, HCAN/HCACON experiments confirm the ${ }^{15} \mathrm{~N}$ chemical shift. Threonine, as discussed earlier has a unique ${ }^{15} \mathrm{~N}$ chemical shift was determined readily. The assignments were confirmed using $\mathrm{HNCO} / \mathrm{HNCACO}$, Figure 1.16a. Another glutamine residue in the cross-link module, Q31 has a more upfield amide proton and nitrogen than Q3. CBCANH/CBCACONH in Figure 1.16b shows side chain carbons of Q31 correlated with $i-1$ residue of F32. Note that F32 has very similar spin system as Y13 except that phenylalanine has a more downfield $\mathrm{C} \beta$ chemical shift. Assignments of resolved ${ }^{15} \mathrm{~N}-{ }^{1} \mathrm{H}$ HSQC peaks were verified using the HNN experiment as shown in Figure 1.16c. Most intense peak corresponds to the diagonal and cross-correlated peaks 
are either shown in-phase or anti-phase depending on the $i$ residue. Residues except proline and glycine are depicted as positive diagonal peaks whereas glycine appears as negative diagonal peak. Moreover, the former yields a negative cross-correlated peak $(i-1$ and $i+1)$ whereas the latter has negative $i-1$ and positive $i+1$. For the QFG sequence with F32 as the $i$ residue, shown in Figure 16c, diagonal peak was shown as positive peak (red) and $i+1$ and $i-1$ were shown as negative (green). G33 diagonal and $i-1$ peaks were observed to have negative signs whereas $i+1$ residue was shown a positive.

Alanine side chains resonances of the cross-link module show highly degenerate peaks thus $\mathrm{CBCANH/CBCACONH}$ experiments were not as much informative, although, alanines after lysines were identified using this experiment. Side chain $\mathrm{C} \alpha$ and $\mathrm{C} \beta$ of P18 were also assign using the correlation from A19. To assign alanine resonances, $\mathrm{HNCO} / \mathrm{HNCACO}$ experiments were used. For resonances that appear as one broad peak instead of two resolved peaks for $i$ and $i-1$, the $i-1$ resonance (red) was assigned using the HNCO spectra (green) as shown in Figure 1.17. Moreover, the full chemical shift assignment for 2020 and 202424 are reported in Appendix I-C. 

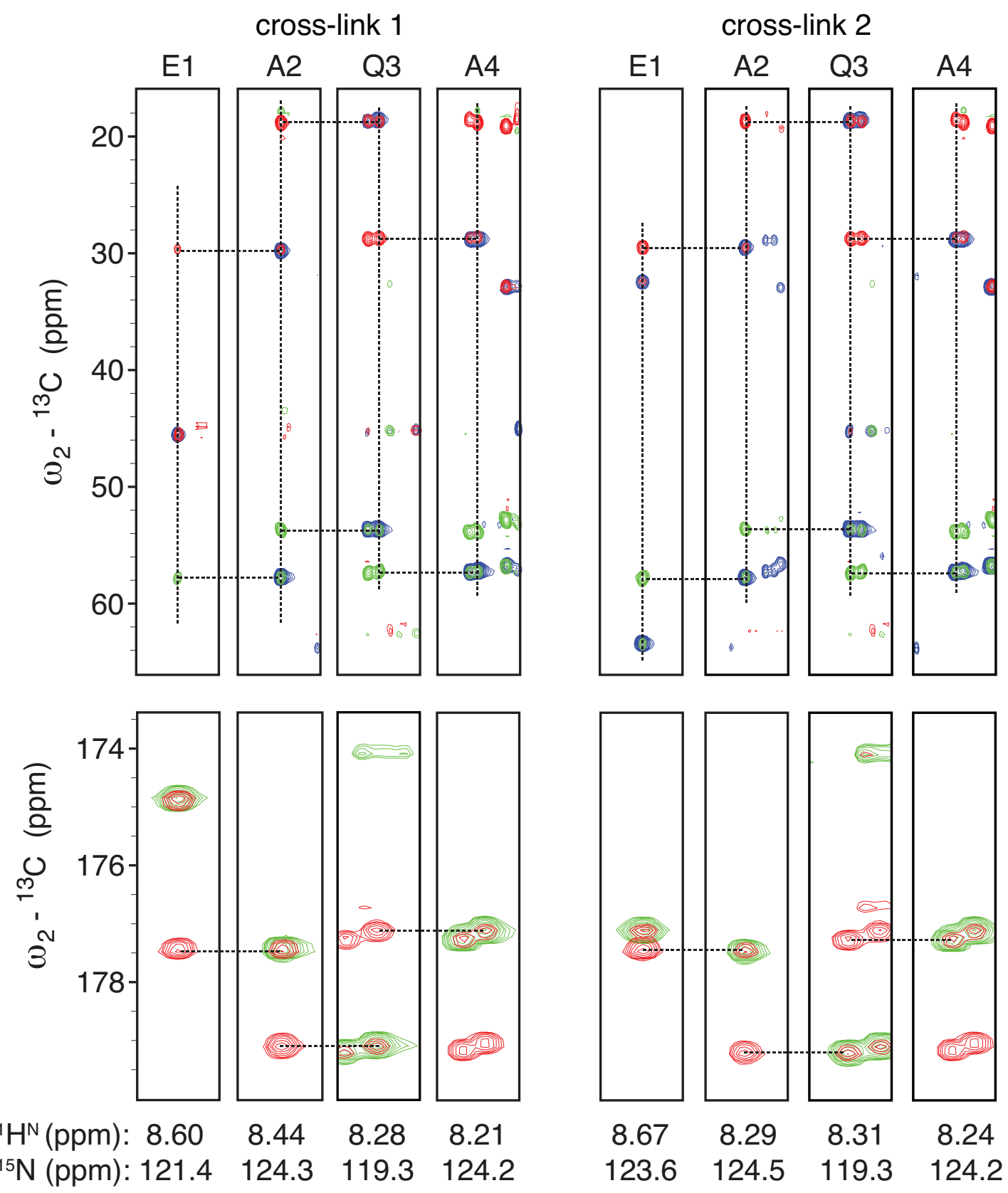

Figure 1.15 CBCANH/CBCACONH (top) and HNCO/HNCACO (bottom) of the first four residues of the cross-link modules of 2020. Cross-links 1 and 2 are found after the hydrophobic modules $20^{\prime}$ and $24^{\prime}$ respectively. 

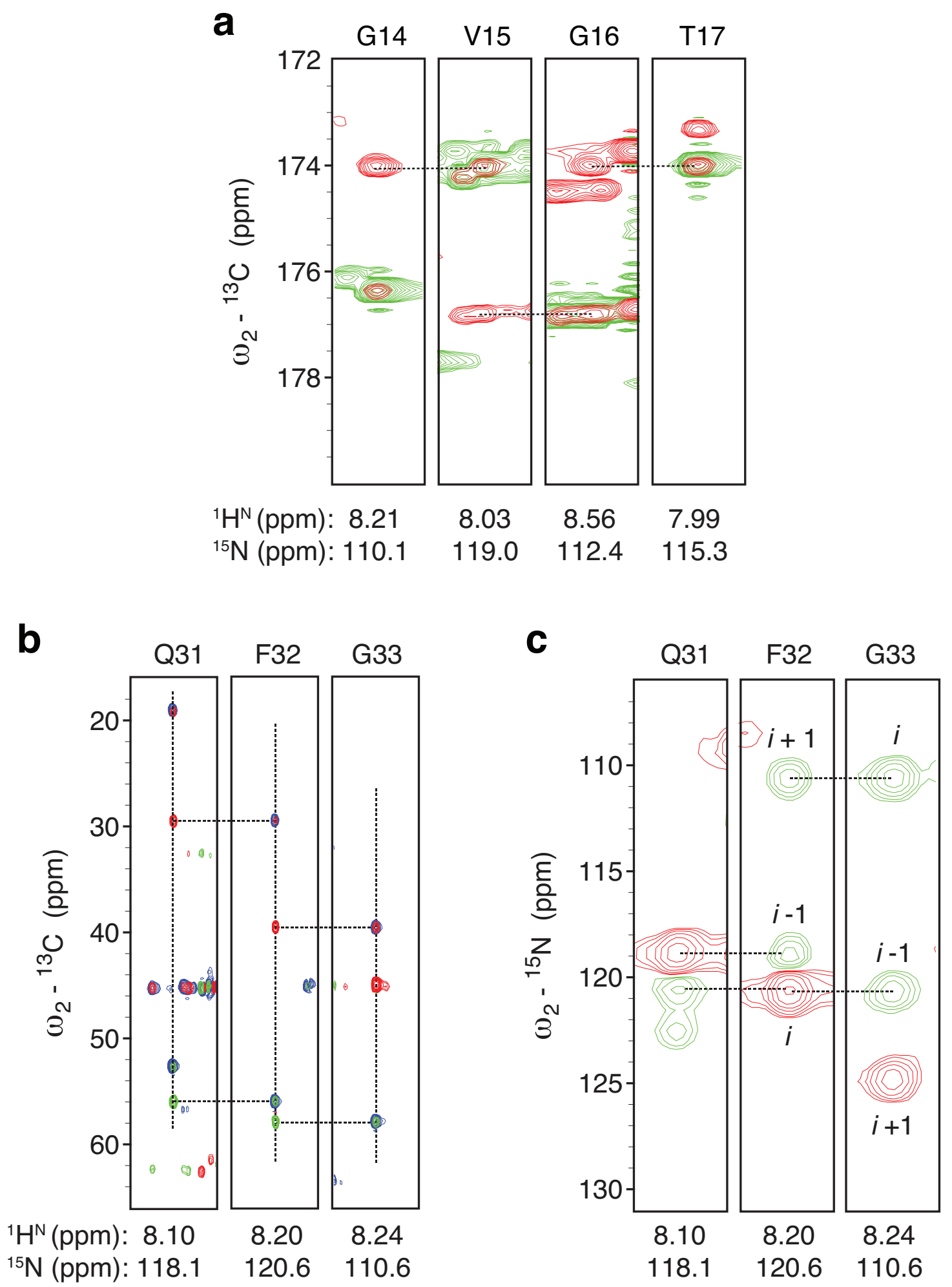

Figure 1.16 (a) HNCO/HNCACO of GVGT (b) CBCANH/CBCACONH and (c) HNN of QFG of the cross-link modules of 2020. 

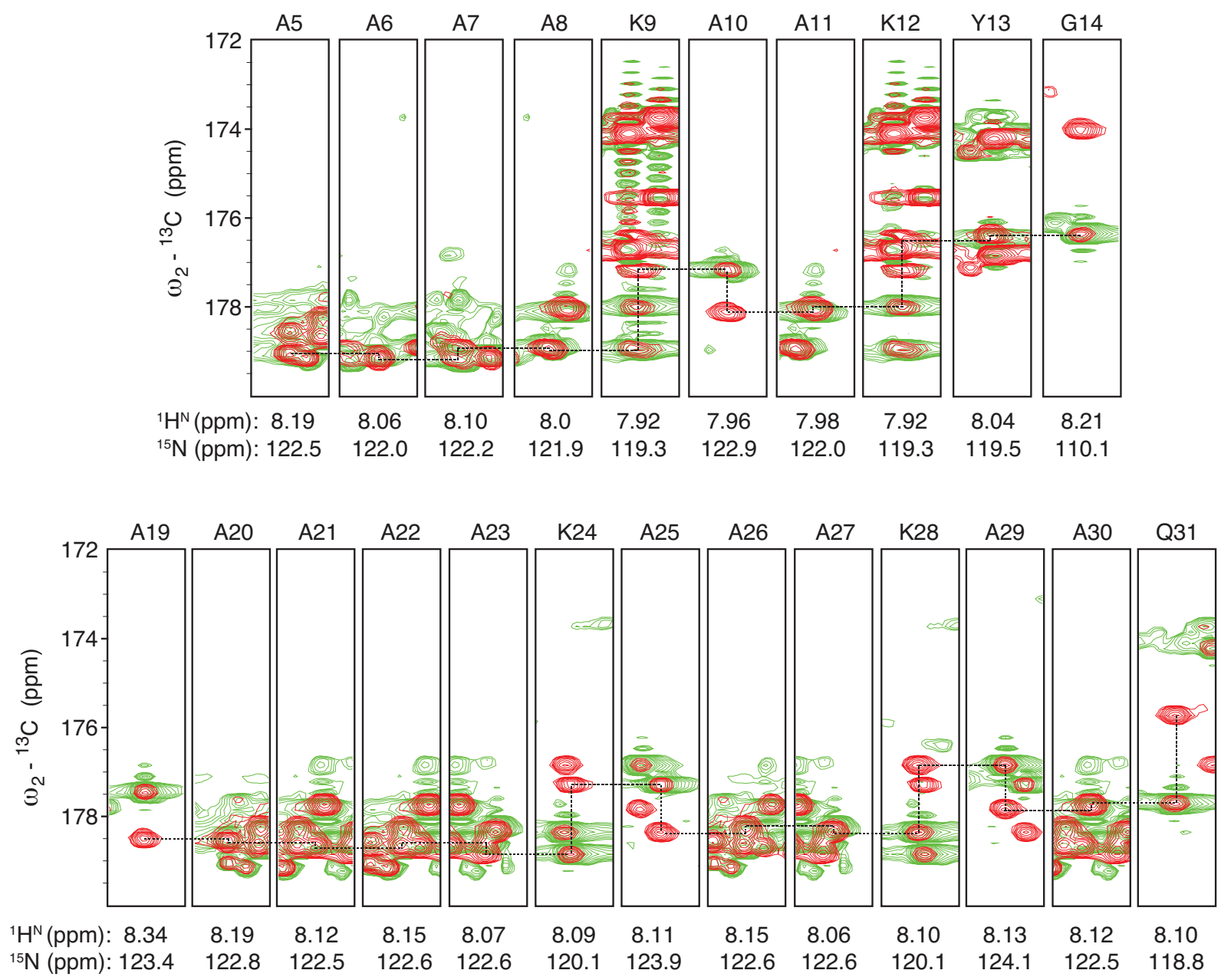

Figure $1.17 \mathrm{HNCO} / \mathrm{HNCACO}$ of alanine-rich cross-link module used to very resonance assignments. $\mathrm{HNCO}$ peaks (green) originates from $\mathrm{CO} i-1$ whereas HNCACO peaks (red) are from $\mathrm{CO}$ of $i$ and $i-1$ residues. 


\subsubsection{Secondary Structure Analysis}

Chemical shifts reported here were compared against the random coil shift values of Poulsen ${ }^{18}$ to obtain the secondary shift $(\Delta \delta)$. Chemical shifts consistent with $\alpha$-helix, $\beta$-sheet and polyproline II (PPII) secondary structures for all constructs were obtained using Sparta.$+{ }^{36}$ Average chemical shifts were calculated and shown in red and blue dashed line in Figure 1.18 for $\alpha$-helix and $\beta$-sheet, respectively. Generally, proteins with positive $C \alpha$ and negative $C \beta$ secondary shifts have an $\alpha$-helical secondary structure ${ }^{17}$ and the opposite is observed for $\beta$-sheet with negative $C \alpha$ and positive $C \beta$ secondary shifts. PPII on the other hand, has $\phi$ and $\varphi$ angles near the region of $\beta$-sheet in Ramachandran plot but adopts a different hydrogen bonding pattern. ${ }^{37}$ PPII secondary shift values are similar to $\beta$-sheet average values and, therefore, are not shown.
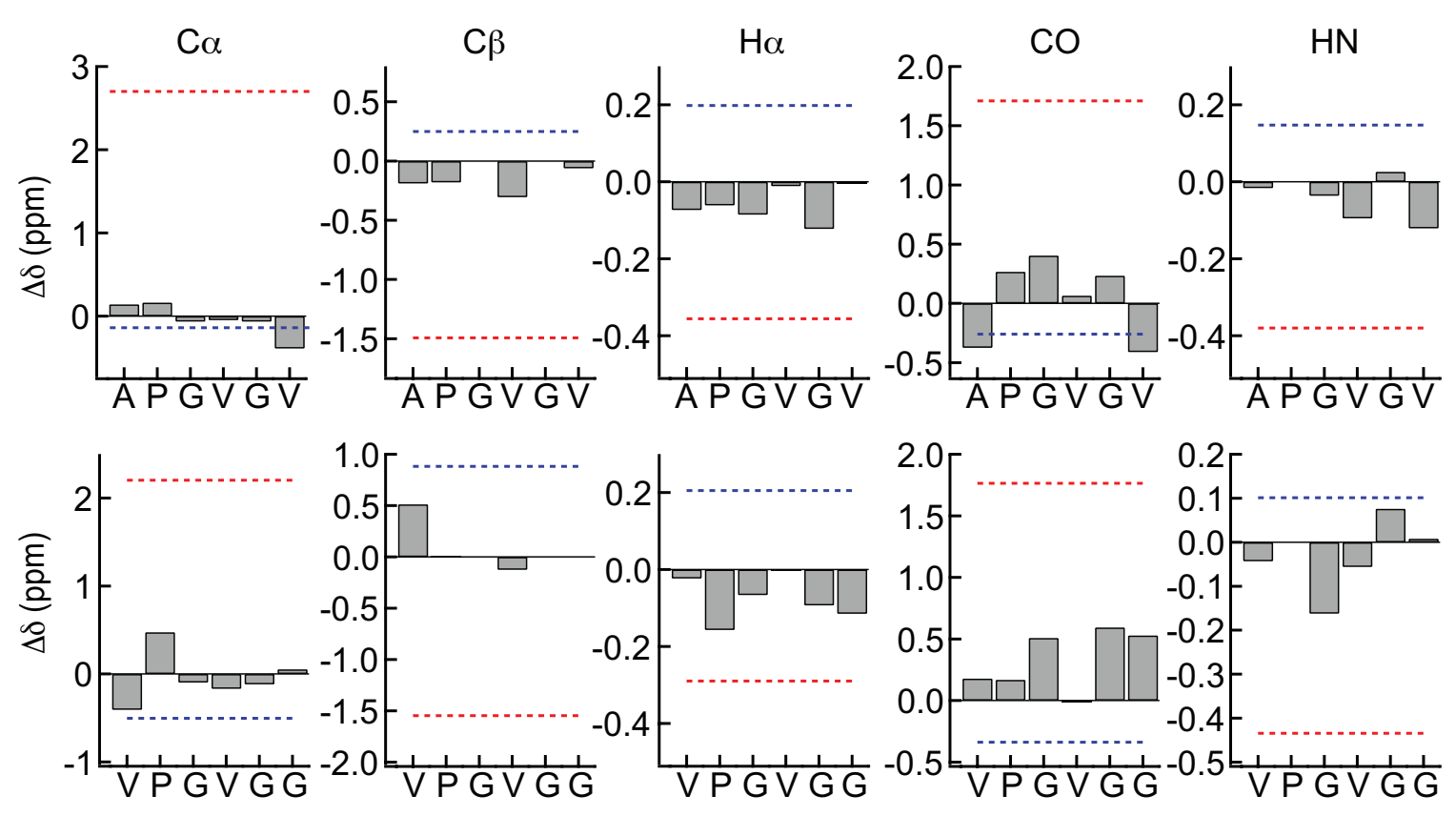

Figure 1.18 Secondary shift analysis of the hydrophobic modules; top panel for $24^{\prime}$ and bottom panel for $20^{\prime}$ in 2020. No distinct chemical shift patterns observed that follows that of average chemical shifts of structured proteins as shown in red and blue dashed lines for $\alpha$-helix and $\beta$-sheet, respectively. ${ }^{30}$ 
Secondary shift analysis of the hydrophobic modules $20^{\prime}$ and $24^{\prime}$ are shown in Figure 1.18. It has been previously shown that the chemical shifts of the hydrophobic modules are conserved in all constructs. Therefore, in these analyses, the chemical shifts obtained from 2020 were used. The experimental chemical shifts (gray bars) for both hydrophobic modules show no consistent pattern that are present in folded proteins. In comparison with the $\alpha$-helix and $\beta$-sheet chemical shifts, the experimental chemical shift values are closer to random coil shift values. Similar analyses were done in the crosslinking sequence of $X$ and $x^{\prime}$ within 2020 and $24 x^{\prime}$ respectively. The changes in chemical shifts were plotted in Figure 1.19 shown as red bars. Both $\mathrm{X}$ and $\mathrm{x}^{\prime}$ have positive $\mathrm{C} \alpha$ secondary shift values whereas $\mathrm{C} \beta$ and $\mathrm{H} \alpha$ are negative. Comparing them with $\alpha$-helix (red) and $\beta$-sheet (blue) average shifts, the $\Delta \delta$ values lean towards $\alpha$-helical value.
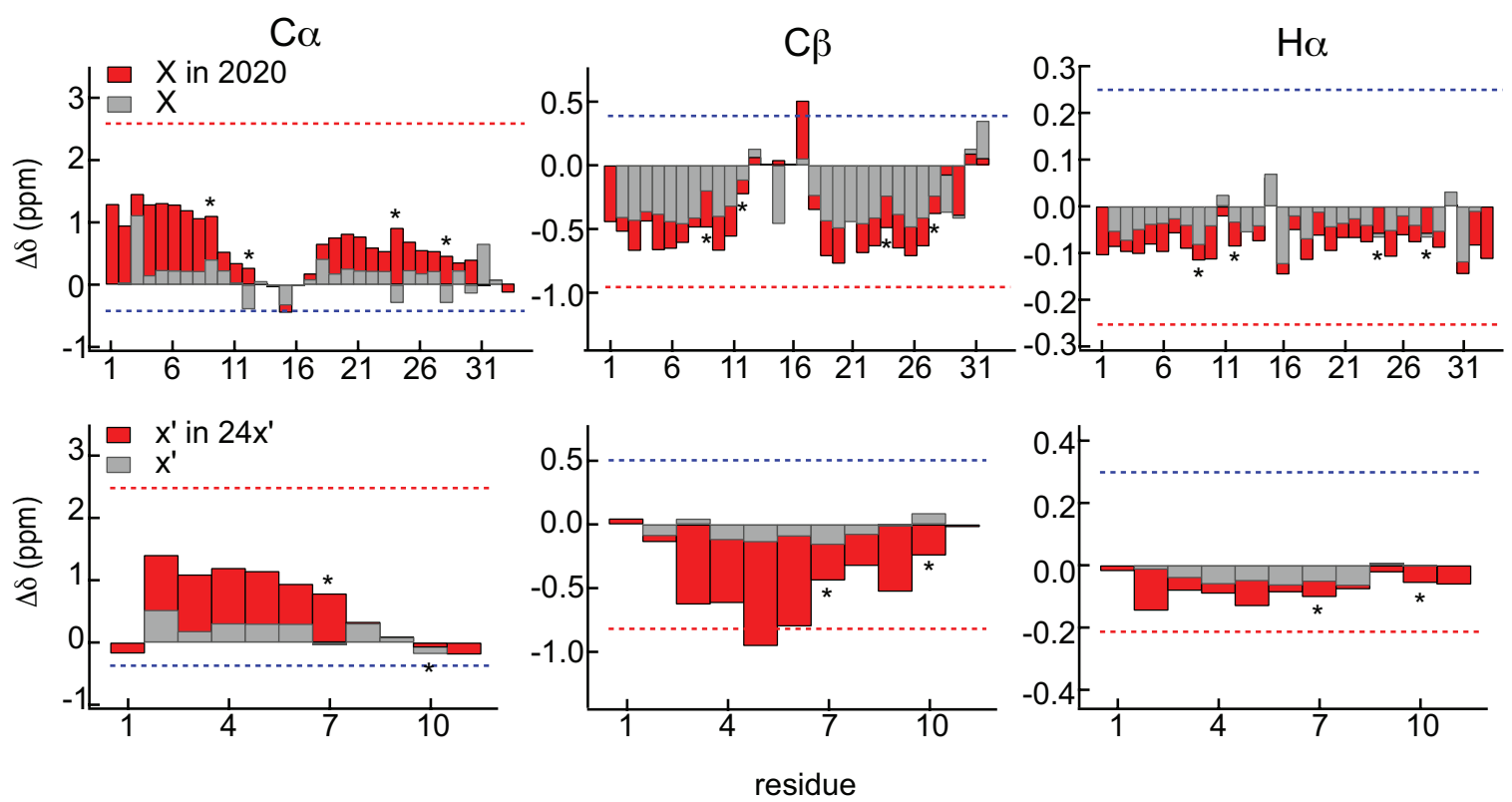

Figure 1.19 Secondary shift analysis of the free cross-linking peptides $\mathrm{X}$ and $\mathrm{x}^{\prime}$ (gray) versus the cross-linking module in 2020 and 24x' (red). Lysine residues are marked with asterisks. $^{30}$ 
This $\alpha$-helical trend is observed in the polyalanyl region and leans toward random coil in the termini, and in the GVGTP sequence as well, in X. However, the magnitude of the $\Delta \delta$ does not reach the values for $\alpha$-helix. This indicates presence of a weak $\alpha$-helical or a transient $\alpha$-helical secondary structure. Moreover, when $\Delta \delta$ between the free crosslinking peptide (shown as gray bars) and the cross-linking module in the constructs are compared, it is evident that these $\alpha$-helical tendencies have increased with the latter. This difference was observed with the ${ }^{15} \mathrm{~N}-{ }^{1} \mathrm{H}$ HSQC spectrum of the free peptide versus the cross-link module within the construct having different chemical shifts. Tamburro and coworkers proposed that the cross-linking modules are in fact not $\alpha$-helical in aqueous solutions but display an $\alpha$-helical structure in a hydrophobic environment. ${ }^{22}$ In this case, an increase in $\alpha$-helical secondary structure are observed when these cross-linking modules are flanked with the hydrophobic modules. An increase in hydrophobic environment also occurs upon temperature-induced phase transition or when peptides are dissolved in TFE. ${ }^{22}$

Recently, a construct similar to $24 \mathrm{x}^{\prime}$ with simplified cross-link and hydrophobic construct was investigated by Reichheld and coworkers. ${ }^{38}$ They concluded that the crosslink module in their construct are disordered. The cross-link modules in their construct were found at the termini and the middle of the sequence. This supports the hypothesis that the cross-link modules only adopt $\alpha$-helical conformation when flanked with hydrophobic domains.

Aside from secondary shifts, NOE patterns are indicative of secondary structures. $3 \mathrm{D}{ }^{15} \mathrm{~N}$-edited NOESY-HSQC paired with ${ }^{15} \mathrm{~N}$-edited TOCSY-HSQC experiments were used to show intra- and inter residue NOE cross-peaks. From this time forward, the focus 
of the secondary structure analysis will be on the simplest construct, $24 \mathrm{x}^{\prime}$, as it has been shown that the hydrophobic and cross-link modules have very similar characteristic on all constructs.

NOE intensities are correlated to approximately inverse sixth power of the nuclei distances $\left(\frac{1}{r^{6}}\right)$, in this case, proximity of the amide protons to other protons. NOE can only be observed with nuclei distances up to $5 \AA$; thus, nuclei distances can be estimated using this experiment. For hydrophobic modules, ${ }^{1} \mathrm{H}-{ }^{1} \mathrm{H}$ NOE of the $i-1$ and $i$ side chains correlated with $\mathrm{HN}$ were observed. The ratio of the NOE of $\alpha \mathrm{N}_{\text {seq }} / \alpha \mathrm{N}_{\text {intra }}$ peak intensities plotted in Figure 1.20 shows that the preceding side chain peaks are more intense than the
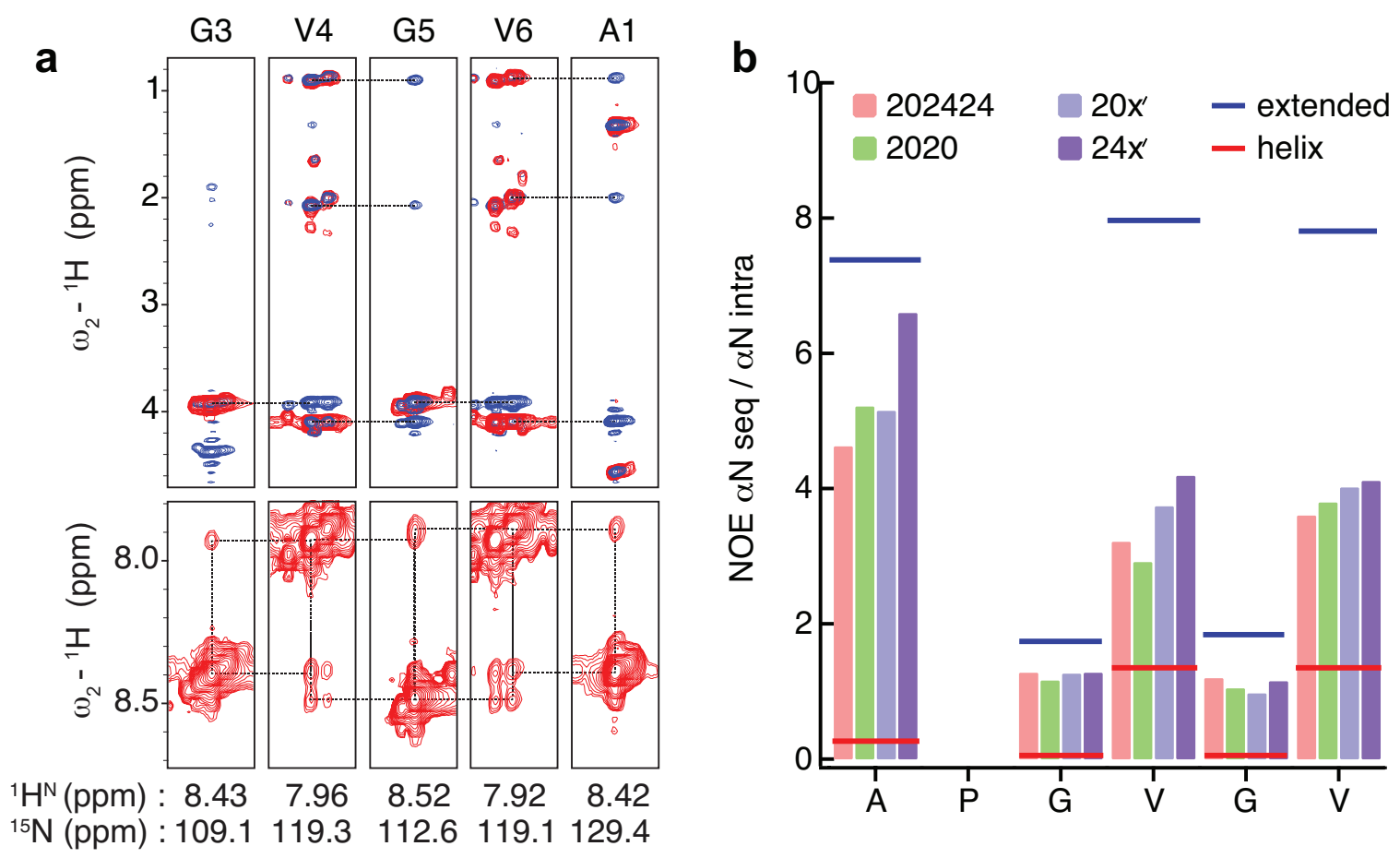

Figure 1.20 (a) ${ }^{15} \mathrm{~N}$-edited NOESY-HSQC (red) overlaid with ${ }^{15} \mathrm{~N}$-edited TOCSY-HSQC of $24^{\prime}$ in $24 \mathrm{x}^{\prime}$. (b) NOE ratios of sequential and intraresidue $\alpha \mathrm{N}$ of $24^{\prime}$ found in four different constructs. 
intraresidue side chain, suggesting an extended structure. ${ }^{27}$ The residue before the prolyl have the highest ratio indicating that $\mathrm{V} 6-\mathrm{A} 1 \alpha \mathrm{N}(i-1, i)$ are relatively closer to each other than the rest of the residues in the hydrophobic module. Note that NOE ratios for proline were not calculated because of lack of amide proton. The NOE ratios were compared with the values for $\alpha$-helix and $\beta$-sheet, as shown in the Figure $1.20 \mathrm{~b}$.

For cross-linking module, note that the $\mathrm{H} \alpha$ and $\mathrm{H} \beta$ of alanyl residues have the same chemical shifts, therefore, no $\alpha \mathrm{N}(i-1, i)$ values were obtained. However, the HNHN NOE can be observed for peaks resolved in proton dimension. This is true for the alanine in $\mathrm{x}^{\prime}$ which are more defined than in $\mathrm{X}$. In $\mathrm{x}^{\prime}, \mathrm{NN}(i, i+1)$ can be observed which is a characteristic of an $\alpha$-helix, Figure 1.21. Stronger peak intensities are also observed for the HN-HN NOE in the cross-linking than in the hydrophobic module. This shows that the residues in $\mathrm{x}^{\prime}$ are closer to each other than residues in the hydrophobic region. It also supports the result from the secondary shift data analysis that the cross-linking module

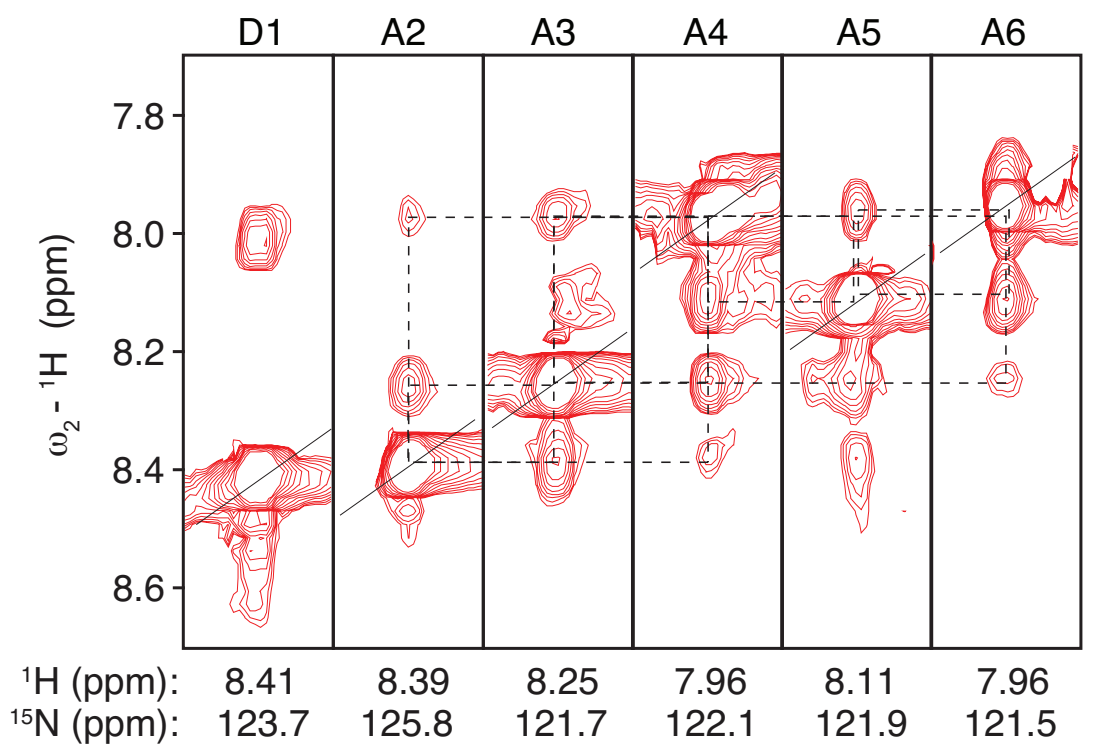

Figure $1.21{ }^{15} \mathrm{~N}$-edited NOESY-HSQC of the cross-link residues showing NOE contacts between amide protons. ${ }^{30}$ 
has weak $\alpha$-helical secondary structure. An $\alpha$-helix has a compact structure; therefore, more NOE peaks are expected. Lysyl NOEs are not obtained because of the spectral overlap with the valines in the hydrophobic module.

An important characteristic of secondary structure is the presence of hydrogen bonding to stabilize the structure. To probe for the presence of hydrogen bonds, the temperature coefficients of the amide protons were obtained, Figure 1.22a. For 24x', $\Delta \delta_{\mathrm{HN}} / \Delta \mathrm{T}$ of residues in hydrophobic module are in average $-7.8 \pm 1 \mathrm{ppb} / \mathrm{K}$, consistent with the random coil values of $<-6.5 \mathrm{ppb} / \mathrm{K} .{ }^{39}$ Residues in the cross-linking module have temperature coefficient range of -8.2 to $-3.8 \mathrm{ppb} / \mathrm{K}$ as summarized in Figure $1.22 \mathrm{~b}$. Notably, alanines adjacent to lysine residues have values more positive than $-4.6 \mathrm{ppb} / \mathrm{K}$ which is generally attributed to presence of hydrogen bonding. ${ }^{29}, 40$ These are all consistent with the cross-link module having a weak helical characteristic and the hydrophobic module being disordered.

Lysyl residue peaks were not resolved in ${ }^{15} \mathrm{~N}-{ }^{1} \mathrm{H}$ HSQC, thus, temperature dependence of the HN shift cannot be observed. This experiment only works if there are no changes in conformation with increase in temperature ${ }^{41}$ This means that the proteins are stable and do not denature with increasing temperature. To probe this possible change in conformation, ${ }^{13} \mathrm{C}-{ }^{1} \mathrm{H}$ HSQC at different temperatures were obtained, Figure $1.22 \mathrm{c} . \mathrm{C} \alpha$ and $\mathrm{C} \beta$ as well as $\mathrm{H} \alpha$ chemical shifts, as discussed, are good indicators of secondary structure. The ${ }^{13} \mathrm{C}-{ }^{1} \mathrm{H}$ HSQC of $24 \mathrm{x}^{\prime}$ at $20{ }^{\circ} \mathrm{C}, 30{ }^{\circ} \mathrm{C}$ and $40{ }^{\circ} \mathrm{C}$ shows that at these temperatures, no significant change in chemical shift was observed. Hence, at these temperatures, no change in conformation occurs. 


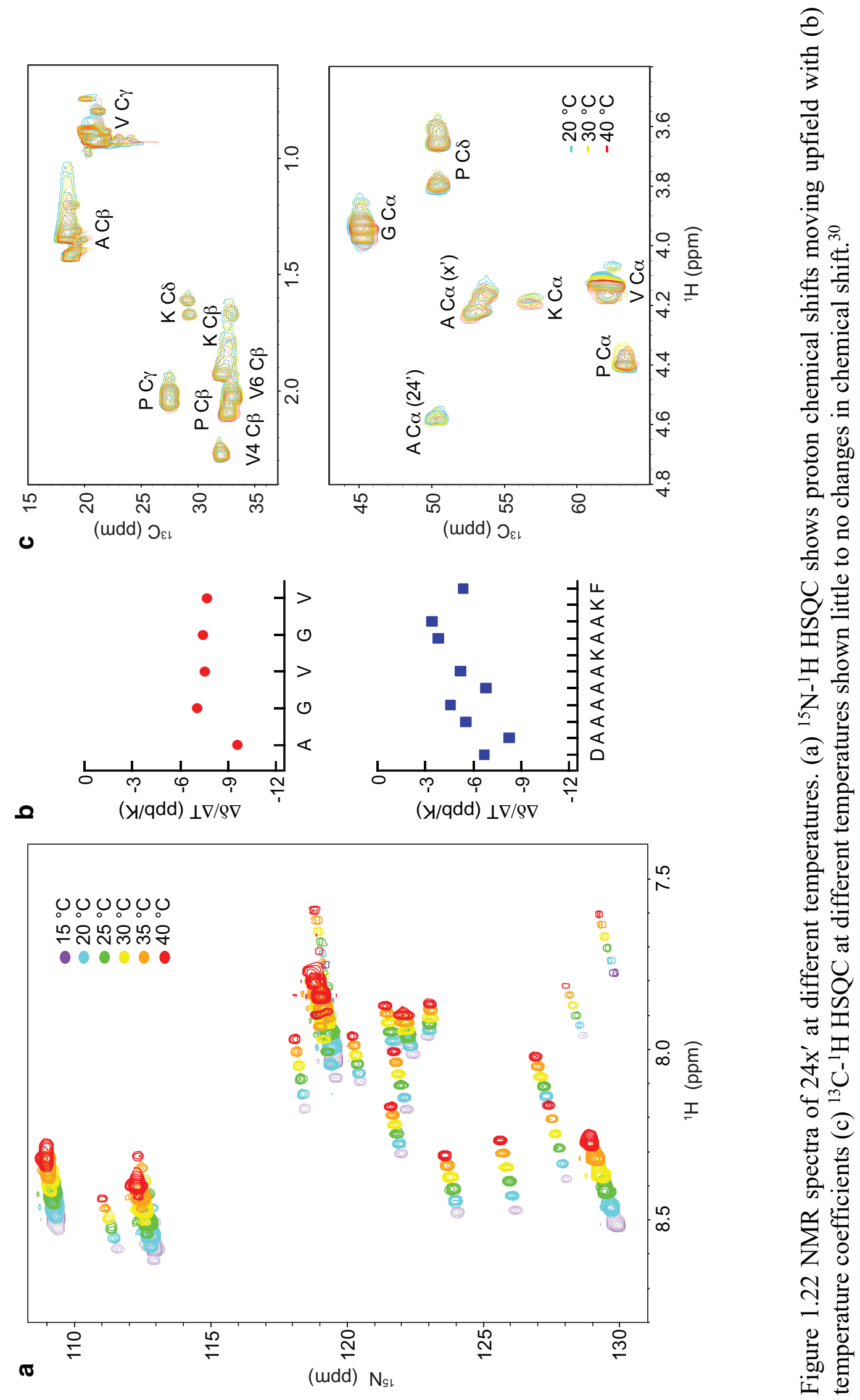


Secondary structures obtained from NMR were complemented with circular dichroism (CD) spectra provided by the Koder lab. Note that a similar curve for 202424 was obtained by Keeley and coworkers ${ }^{11}$ using exon 20-21-23-24-21-23-24. The same was observed with the spectra obtained by Weiss et al. ${ }^{42}$ for exon 24 when compared to 24 '. Simplification of the hydrophobic domain thus, did not affect the average secondary structure of the polypeptides. Figure $1.23 \mathrm{a}$ showed that the addition of $20^{\prime}$ and $24^{\prime}$ to 202424 increased the negative magnitude of the $200 \mathrm{~nm}$ dip as well as that in $220 \mathrm{~nm}$. These can be attributed to an increase in PPII or disordered secondary structure upon addition of hydrophobic domains. Wallace et $a l^{43}$ showed that negative peaks at $190-$ $200 \mathrm{~nm}$ and a positive peak at $220 \mathrm{~nm}$ is a characteristic of PPII whereas disordered structure lacks the latter. Apparently, the spectra of 202424 and 2020 are similar with that of a denatured protein as shown by the characteristic intense negative peak at $200 \mathrm{~nm}$ and a small negative peak at $220 \mathrm{~nm}$. Also, the small peak at $220 \mathrm{~nm}$ can be attributed to a small percentage of $\alpha$-helix and $\beta$-sheet except that at increasing temperature, very small change in the $220 \mathrm{~nm}$ peak is observed. The same results were obtained from CD spectra analysis from Dichroweb ${ }^{44}$ using SELCON3 ${ }^{45}$ and CONTINLL ${ }^{46}$ algorithms. 202424 and 2020 have an estimated secondary structure of $10 \% \alpha$-helix; $27 \% \beta$-sheet; $23 \%$ turns and $40 \%$ disordered. These results are not too far from what Weiss and coworkers ${ }^{47}$ had obtained for the recombinant human tropoelastin. Notably, $20 x^{\prime}$ and $24 x^{\prime}$ have almost the same estimated secondary structure except that the it lacks the distinct negative peak at $220 \mathrm{~nm}$ as shown in Figure 1.23b. Moreover, the CD spectra of 202424 and $24 \mathrm{x}^{\prime}$ at increasing temperature (Figures $1.23 \mathrm{c}$ and $1.23 \mathrm{~d}$ ) show a very little change at 


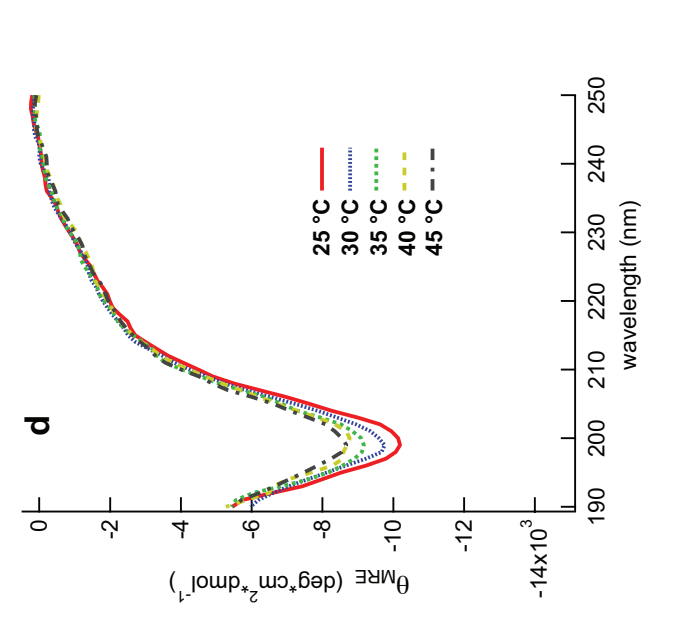

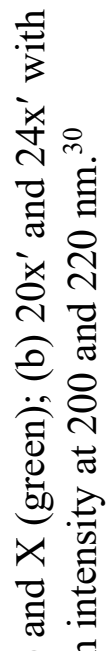

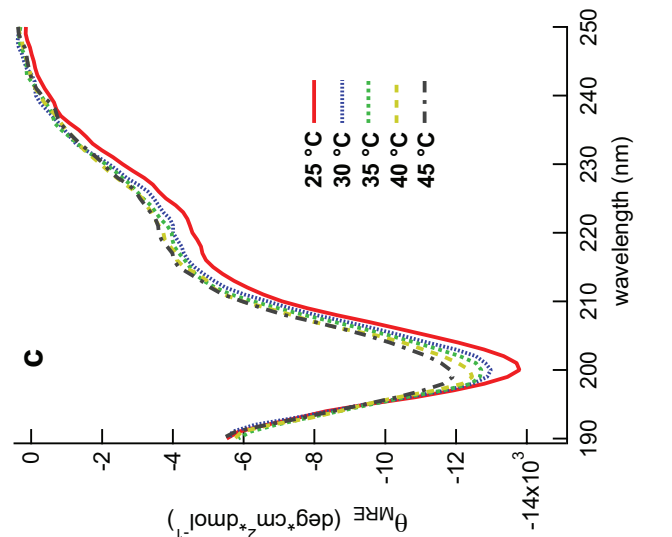

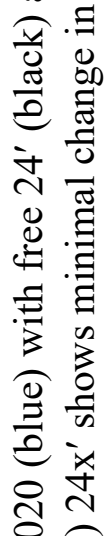

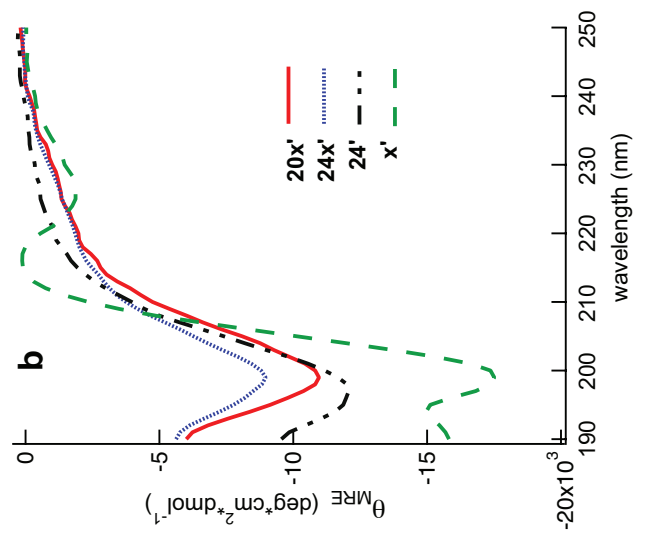

위

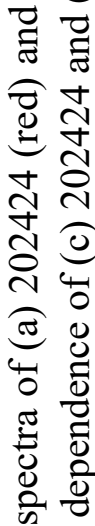

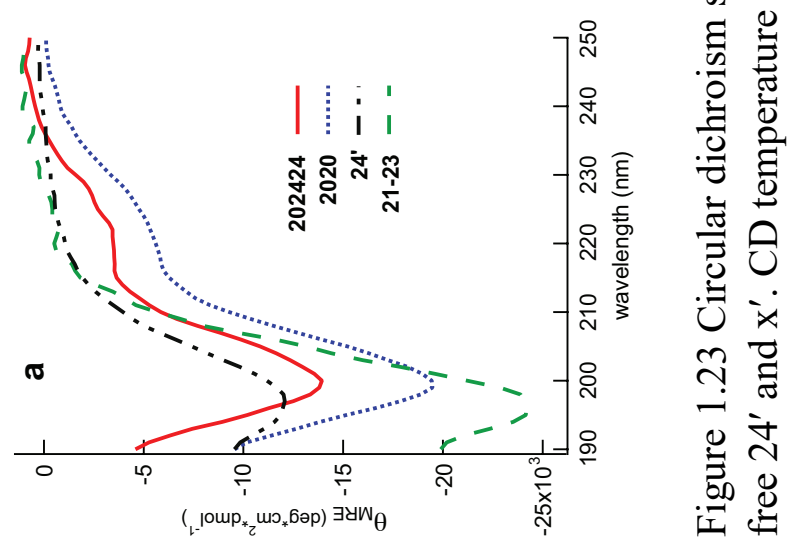


$200 \mathrm{~nm}$ which is attributed to the denaturation of PPII at increasing temperature. These small changes observed in the $\mathrm{CD}$ spectra suggest that at increasing temperature, no disruption of intramolecular interactions occurs.

In this chapter, elastin-like constructs are shown to be disordered with transient $\alpha$ helical secondary structure. Spectra from 2D and 3D NMR experiments show chemical shift averaging of the hydrophobic modules that is indicative of disordered nature. Chemical shift analysis presented not consistent pattern suggesting the absence of secondary structure. Moreover, the secondary structure of the cross-link module is observed only when flanked with hydrophobic modules. Chemical shift analysis revealed secondary shift patterns consistent with $\alpha$-helical secondary structure. These patterns were found in both short (11 residues) and long (33 residues) cross-link modules. Moreover, CD spectra are in agreement with the NMR observations. These minielastin constructs displayed very similar CD spectra as tropoelastin in solution suggesting structural similarity between minielastin and tropoelastin. 


\title{
CHAPTER 2. MINIELASTINS ARE MORE COMPACT THAN TYPICAL IDPS AND UNDERGO LIQUID-LIQUID PHASE TRANSITION LIKE
}

\author{
TROPOELASTIN
}

\subsection{Introduction}

In this chapter, the effect of the sequence of minielastin on the hydrodynamic radius and the temperature of the inverse transition, known as coacervation, were explored. These properties are closely related to assembly of the elastic matrix and the mechanism of elastic recoil in the mature elastin matrix. Secondary shift analysis from the previous chapter shows that minielastins are disordered. Likely due to low sequence complexity and high proline content. ${ }^{48}$ Disordered proteins, such as minielastins, have a relatively simple, flat energy landscape $e^{49-50}$ allowing a dynamic interconversion of conformations. However, the lack of secondary structure does not necessarily inhibit the formation of more compact structures found in folded proteins which have predominantly stable secondary structure.

Compaction can be depicted by disordered homopolymers dissolved in a bad solvent, i.e., when protein:protein interaction is more favorable than protein:solvent interaction. ${ }^{51-52} \mathrm{MD}$ simulations of $\mathrm{Li}$ and coworkers show that the elastin-like polypeptide, (VPGVG) ${ }_{18}$, adapts a compact state in aqueous solution without forming a 
hydrophobic core from which water is excluded..$^{53}$ Their simulations indicate that the systematic distribution of proline residues prevented the formation of the hydrophobic core and stable secondary structure. Moreover, Rauscher ${ }^{54}$ and Kozel ${ }^{55}$ showed that if an elastin-like polypeptide with naturally occurring prolines PGVGVA and PGVGV repeats is mutated to GGVGVA and GGVGV, respectively, they adapt a $\beta$-sheet secondary structure proving that proline is necessary to prevent the formation of amyloid-like fibers. ${ }^{12,53,56}$ Based on MD simulations, the distinction between amyloid-like and elastinlike polypeptides is based on the proline and glycine content and that these residues regulate the hydration and conformational disorder of the monomeric protein. ${ }^{54}$

In this study, systematic variations in the transition temperatures and the hydrodynamic radii were found with respect to the sequence. Briefly summarized, increasing the length of the hydrophobic module, decreasing the length of the cross-link module or increasing the hydrophobicity of the hydrophobic repeat decreases the coacervation temperature. Also, these minielastins are more compact than other IDPs of the same molecular weight including elastin-like proteins that lack cross-link modules. Unlike most materials that expand with increased temperature, these minielastins become more compact, i.e., the hydrodynamic radius decreases as the temperature is increased and the slope of the decrease varies systematically with the length of the minielastin. These results are important in modulating the temperature-responsive properties of elastin-based biomaterials.

Pressure-induced decrease in hydrodynamic radii of minielastin constructs suggests that at high pressure, minielastins sample conformations with lower total volume than at atmospheric pressure and that if void volume is present in minielastins in solution, 
this volume is smaller than the hydration volume. The effects of temperature and pressure on the proton chemical shifts and hydrodynamic radii of minielastin constructs were investigated. It is evident that hydration plays a major role in the coacervation process of minielastin constructs.

\subsubsection{Compaction}

Protein folding and compaction are commonly compared to homopolymers in bad solvents wherein the hydrophobic sidechains tend to form a more favorable hydrophobic core and the polar backbones form hydrogen bonding intramolecularly or with surrounding water. ${ }^{52,} 57$ Scaling rules of polymer physics have been used to relate hydrodynamic radii of folded, denatured ${ }^{52}$ and disordered proteins ${ }^{58-59}$ to its number of residues, $(\mathrm{N})$, via $\mathrm{R}_{\mathrm{H}}=\mathrm{R}_{0} \mathrm{~N}^{v}{ }^{60}$ The exponent, $v$, depends on the conformation of the polymer with values of $\sim 2 / 3$ for the expanded state, $1 / 2$ for theta conditions and $1 / 3$ for folded globular proteins. ${ }^{61-62}$ Flory defined theta conditions as a polymer dissolved in a solvent in which protein-solvent and protein-protein interactions have equivalent strengths. Under these conditions, a flexible protein chain executes a random walk and behaves like a gaussian chain. Folded globular proteins are compact and the hydrodynamic radii are proportional to $\sim \mathrm{N}^{1 / 3}$ i.e., the protein is like an organic molecule packed in a crystalline solid. Proteins in the expanded state have some residual secondary structure but the absence of a compact folded state. The pre-exponential factor, $\mathrm{R}_{0}$, describes the average length of the freely jointed segment of the polymer chain. ${ }^{63}$ It is potentially informative to compare experimentally determined values of $\mathrm{R}_{0}$ with the pre-exponential factor found in folded and chemically denatured proteins. ${ }^{52,58-59}$ 
Marsh and Forman-Kay refined the scaling equation for IDPs to include proline content and net charge in the pre-exponential factor. ${ }^{58}$ Proline favors extended secondary structure found in the PPII helix that has similar phi/psi angles as $\beta$-sheet secondary structure. High net charge, a common characteristic of intrinsically disordered proteins, also tends to favor an extended state due to electrostatic repulsion. Tropoelastin and the minielastins studied here have high proline content in the hydrophobic modules $(\sim 13 \%)$ and net charge due to lysine residues $(\sim 5 \%)$ in the cross-link modules.

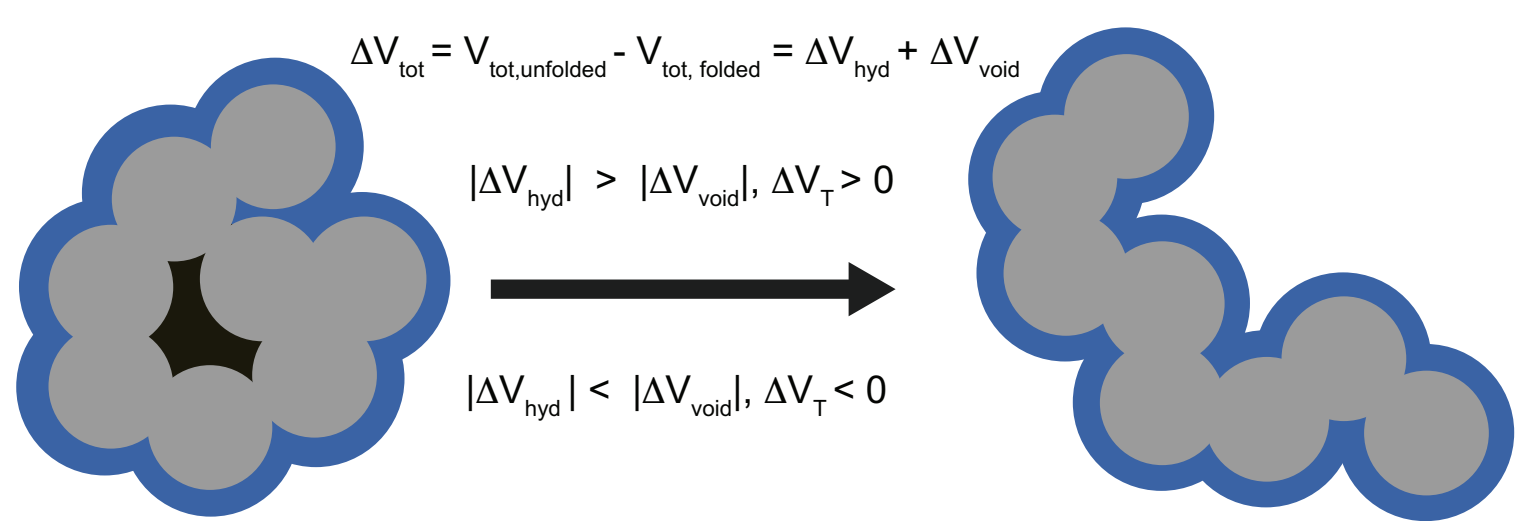

Figure 2.1 The total volume change $\left(\Delta \mathrm{V}_{\text {tot }}\right)$ associated with unfolding of natively folded proteins depend on the balance between the change in void volume $\left(\Delta \mathrm{V}_{\text {void }}\right)$ and the change in hydration volume $\left(\Delta \mathrm{V}_{\mathrm{hyd}}\right)$. Total protein volume is the summation of van der Waals volume $\left(\mathrm{V}_{\mathrm{vdw}}\right.$, gray), void volume (black) and hydration volume (blue). Unfolding results in a decrease in void volume and increase in hydration volume as voids and more molecular surface area become solvent accessible. (Adapted from Chen and Makhatadze, $\left.2017^{64}\right)$

A compact state, as defined in this chapter, is depicted by a low hydrodynamic radius, typically associated with globular, folded proteins. However, hydrodynamic radius is not sufficient in determining the presence or absence of secondary structures in proteins, it provides a general idea on protein's hydrodynamic volume defined by the Stoke's radius $4 \pi \mathrm{R}_{\mathrm{H}} 3 / 3 .{ }^{57}$ Folded states in globular proteins, in general, have greater 
volume than their unfolded state. This is attributed to higher void volume resulting in a negative volume change upon unfolding. ${ }^{65-70}$ Void volume is the space occupied neither by the protein nor the solvent molecules. The total volume of protein in solution is then the summation of van der Waals volume, void volume and hydration volume, Figure 2.1. Van der Waals volume is the volume occupied by the protein atoms. Protein dimensions are affected by thermodynamic parameters, temperature and pressure. Chen and Makhatadze showed that the balance between the void volume and hydration volume are responsible for the pressure-dependence of protein volume whereas temperaturedependence of volume are mostly affected by hydration volume. ${ }^{64,71}$ In this chapter, the effect of temperatures and pressures on hydrodynamic radii of minielastin constructs obtained using PFG NMR were investigated.

\subsubsection{Translational Diffusion}

Measurement of molecular translational diffusion can reveal possible interconverting conformations and protein aggregation. Translational diffusion coefficient, $D_{t}$, describes hydrodynamic properties of diffusing species and is related to the hydrodynamic radius, $\mathrm{R}_{\mathrm{H}}$. The relationship between the hydrodynamic radius and diffusion coefficient is shown in Stokes-Einstein equation,

$$
R_{H}=\frac{k_{B} T}{6 \pi \eta D_{t}}
$$

where $k_{\mathrm{B}}$ is the Boltzmann constant, $T$ is the absolute temperature and $\eta$ is the solvent viscosity. $R_{H}$ describes the diffusion of hard spheres diffusing at the same rate as the protein of interest, but nonetheless provide a good estimate of protein size when compared with the $\mathrm{R}_{\mathrm{H}}$ of folded and denatured proteins with same number of residues. ${ }^{52}$ 
$\mathrm{R}_{\mathrm{H}}$ is determined using various biophysical technique such as pulsed field gradient NMR $(\mathrm{PFGNMR})^{52}$, small angle X-ray scattering $(\mathrm{SAXS})^{72}$, size exclusion chromatography (SEC), dynamic light scattering (DLS) ${ }^{73}$ and analytical ultracentrifuge (AUC). ${ }^{74}$ Toonkool et al. used PFGNMR and AUC to measure the hydrodynamic radius of recombinant human tropoelastin. Using AUC, they have demonstrated that two interconverting conformations of monomeric species of tropoelastin exist whereas PFGNMR showed the weighted average $R_{H}$ of the diffusing species. ${ }^{75}$ Translational diffusion coefficients are extracted by applying pulsed field gradients of increasing strength. Stejskal-Tanner, eq. 2.2, shows the effect of diffusion on signal attenuation at constant diffusion time, $\Delta$ and gradient length, $\delta . \gamma$ is the gyromagnetic ratio of nuclei being observed. The plot of the squared of gradient strength and the natural logarithm of signal attenuation yields a linear correlation. ${ }^{76}$

$$
\mathrm{S}(2 \tau)=\mathrm{S}(0) \exp -\gamma^{2} \mathrm{D} \delta^{2}\left(\Delta-\frac{\delta}{3}\right) \mathrm{g}^{2}
$$
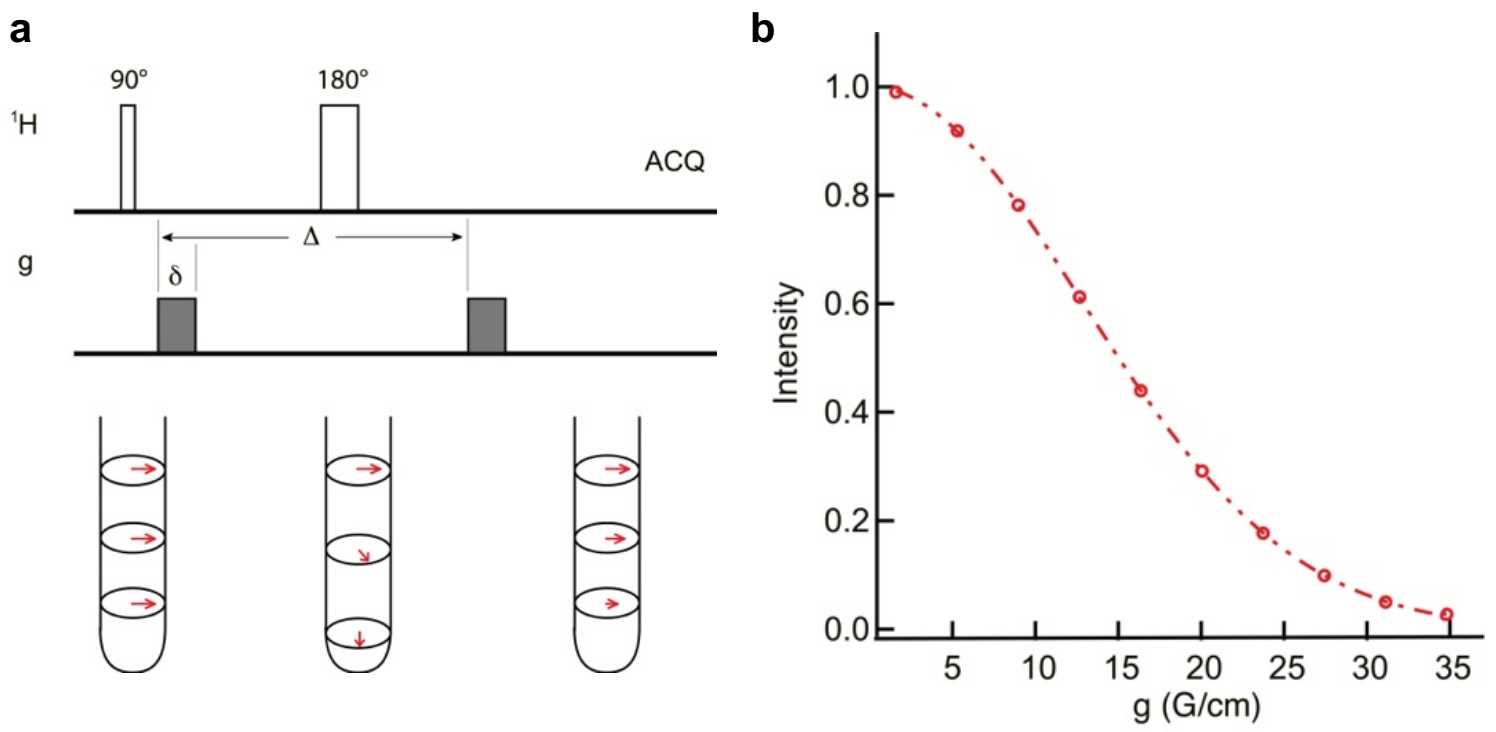

Figure 2.2 (a) Pulsed Field Gradient Spin Echo (adapted from Johnson, 199977) and representative magnitude of signal in each step of the pulse sequence. (b) Plot of signal intensity with increasing field strength is a gaussian curve. 
Figure 2.2 shows a general schematic of a PFGNMR experiment. A $90^{\circ}$ pulse is applied to generate transverse magnetization then a gradient encodes the position of the nuclei. After some time, a second pulse is applied to refocus the transverse magnetization. After the delay time $\Delta$, a second gradient is applied to decode the position of the nuclei. Diffusion causes incomplete refocusing of the transverse magnetization resulting a decrease in signal intensity. Gradient strength is proportional to encodingdecoding efficiency. Stronger gradients are more sensitive to small changes in position thus, higher signal attenuation is observed.

\subsubsection{Coacervation}

Coacervation is described as the formation of a dense viscoelastic liquid as it undergoes an inverse temperature transition. Inverse temperature transition as defined by Urry is the occurrence in which in a multicomponent system, one component decreases entropy with increase in temperature. ${ }^{20}$ To form the mature elastin, tropoelastin is transported to the extracellular matrix of the cell. In these conditions, tropoelastin is known to attach to microfibrils and coacervate which facilitate cross-linking with the presence of lysyl oxidase. ${ }^{78-79}$ Therefore, the unique property of these proteins to undergo inverse temperature transition is crucial in formation of bioelastic materials. Keeley and coworkers have shown that minielastins, tropoelastin and elastin-like polypeptides that only contain hydrophobic modules, ${ }^{80}$ all undergo coacervation. ${ }^{12-14,81}$ The first stage of coacervation is the reversible formation of the liquid-liquid phase and the second phase is the irreversible maturation with observed formation of fibrillar proteins. ${ }^{82}$ 
For recombinant human tropoelastin, coacervation occurs at $37^{\circ} \mathrm{C}, 150 \mathrm{mM} \mathrm{NaCl}$ and $\mathrm{pH} 7-8 .{ }^{47}$ Several factors such as molecular weight, hydrophobicity, salt and protein concentration has been known to affect coacervation temperature $\left(\mathrm{T}_{\mathrm{c}}\right) .{ }^{14,80,83}$ Thus, protein molecular weight and hydrophobicity can be manipulated to program an elastinlike polypeptide to coacervate at the desired temperature. Keeley and coworkers have reported coacervation curves for various recombinant elastin polypeptides at different salt and protein concentration with constructs of various hydrophobicity and molecular weights. ${ }^{12}$ Increase in salt and protein concentration caused a decrease in $T_{c}$ whereas sequence length and hydrophobicity relationship with $\mathrm{T}_{\mathrm{c}}$ were not fully defined. 


\subsection{Materials and Methods}

\subsubsection{Pulsed Field Gradient NMR}

NMR samples used in all PFG NMR experiments are described in the previous chapter. Diffusion constants were measured on Varian $700 \mathrm{MHz}$ equipped with HCN cryoprobe. PFG NMR was conducted using the bipolar pair pulse stimulated echo (dbppste) pulse sequence in the Varian NMR DOSY package. ${ }^{84}$ Gradients were calibrated against the known diffusion constants of three samples, $1 \% \mathrm{H}_{2} \mathrm{O}$ in $\mathrm{D}_{2} \mathrm{O},{ }^{85}$ ubiquitin ${ }^{86}$ and lysozyme. ${ }^{87}$ Signal decay, S, as a function of gradient strength, g, was fit to a Gaussian function, $S=S_{0} \exp \left(-\mathrm{g}^{2} / \mathrm{w}^{2}\right)$ and diffusion coefficient was determined from the equation $\mathrm{w}^{-2}=\mathrm{D} \gamma^{2} \delta^{2}(\Delta-\delta / 3-\tau / 2) .{ }^{77}$ Diffusion time, $\Delta$ and gradient length, $\delta$ were optimized to cover from $5 \%$ to $95 \%$ signal attenuation as shown in Figure 2.3 with optimum values $\Delta=134.5 \mathrm{~ms}$ and $\delta=5 \mathrm{~ms}$. Twenty $1 \mathrm{D}$ interleaved spectra were obtained with gradient strength ranging from 1.6 to $36.7 \mathrm{G} / \mathrm{cm}$ and 16 scans each spectrum.

The diffusion constant was determined in triplicate at each temperature to confirm thermal equilibration and measured again at the starting temperature, $25^{\circ} \mathrm{C}$, following each temperature change to confirm reversibility as shown in Figure $2.3 \mathrm{~b}$. The standard deviation of the multiple determinations at $25{ }^{\circ} \mathrm{C}$ was used as the experimental uncertainty in the diffusion constants. Hydrodynamic radii, $\mathrm{R}_{\mathrm{H}}$, were calculated using the Stokes-Einstein relation, $D=k_{B} T / 6 \pi \eta \mathrm{R}_{\mathrm{H}}$, with the known viscosity of water at each temperature. $^{88}$ 


\subsubsection{High-pressure NMR}

High-pressure NMR experiments were conducted using Bruker Avance III HD $800 \mathrm{MHz}$ NMR spectrometer equipped with 5-mm inverse TCI cryoprobe. Samples were placed in a ceramic tube with $3 \mathrm{~mm}$ inner diameter attached to a syringe pump (Daedalus Innovations LLC, Aston,PA). $300 \mu \mathrm{L}$ of mineral oil was added to act as a barrier between the sample and the hydrostatic pressure source. Spectra were obtained at pressures of 202500 bar with pressures cycled to 20 bar between each pressure measurement to ensure reversibility. The Bruker pulse sequence utilized a double-stimulated echo with bipolar gradients (dstebpg3s), a diffusion time of $107 \mathrm{~ms}$ and gradient length of $5 \mathrm{~ms}$. Spectra were acquired with 16 scans interleaved with 16 gradient strengths from 1 to $51 \mathrm{G} / \mathrm{cm}$.

PFG NMR hydrodynamic analyses were confirmed by AUC experiments done in collaboration with Dr. William Dean (University of Louisville School of Medicine). $\mathrm{R}_{\mathrm{H}}$

a

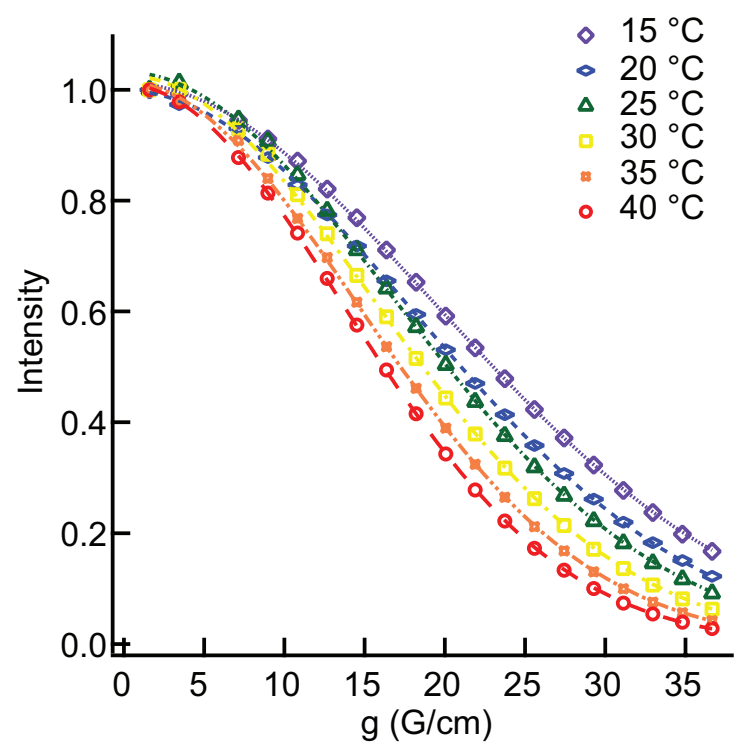

b

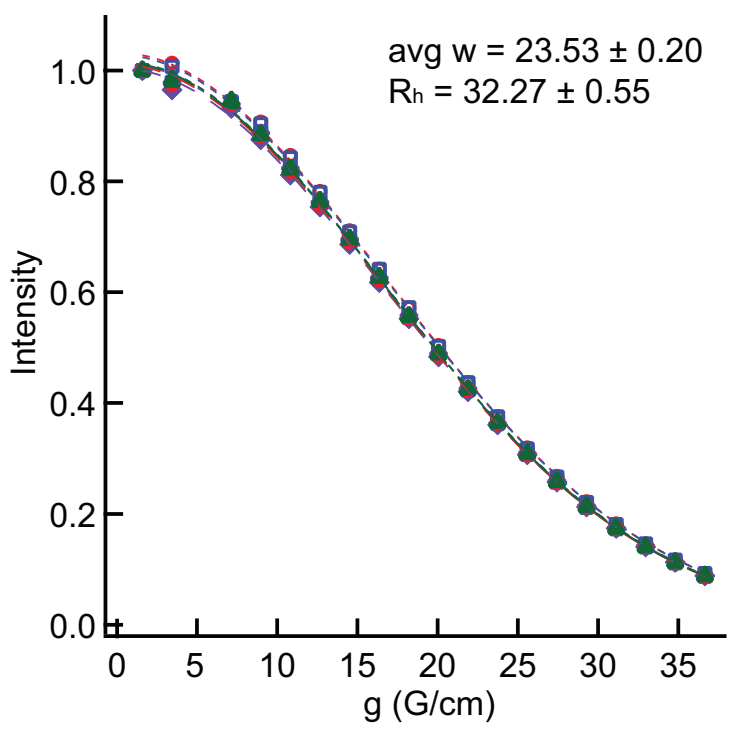

Figure 2.3 Normalized signal intensities were plotted versus the gradient strength at a) different temperatures and b) different trials at $25{ }^{\circ} \mathrm{C}$ of 202424 shows the reversibility and reproducibility of the diffusion experiments. 
values obtained using sedimentation velocity experiment were consistent with NMR results as shown in Table 2.1. Protein monomers were observed to have frictional ratio $f / f_{0} \sim 2$. Frictional ratio denotes the deviation of molecules from an ideal hard, unhydrated sphere. This idealized sphere has a value of $1 .{ }^{89}$ Thus, greater than 1 value suggests asymmetry and hydration. The value of $f / f_{0}$ for tropoelastin, Toonkool et al. ${ }^{75}$ was close to the value of minielastins studied here.

\subsubsection{Coacervation}

Standard coacervation conditions were utilized in the coacervation experiment as described by Keeley and coworkers. ${ }^{11} 25 \mu \mathrm{M}$ protein solution in $\mathrm{pH} 7.5$ Tris buffer, 1.5 $\mathrm{M} \mathrm{NaCl}$ and $1 \mathrm{mM} \mathrm{CaCl}_{2}$ was prepared. The concentrations were calculated from the absorbance at $280 \mathrm{~nm}$ using extinction coefficients, $\varepsilon$ of $13940 \mathrm{M}^{-1} \mathrm{~cm}^{-1}$ for 202424 and 2020 and $11380 \mathrm{M}^{-1} \mathrm{~cm}^{-1}$ for $20 \mathrm{x}^{\prime}$ and $24 \mathrm{x}^{\prime} . \mathrm{T}_{\mathrm{c}}$ is depicted by the onset of turbidity of the solution caused by the liquid-liquid phase transition. The absorbance was monitored at 440 nm using Cary-50 UV-VIS spectrophotometer with a Peltier temperature control accessory at a rate of $0.5^{\circ} \mathrm{C} / \mathrm{min}$ to allow equilibration of the sample.

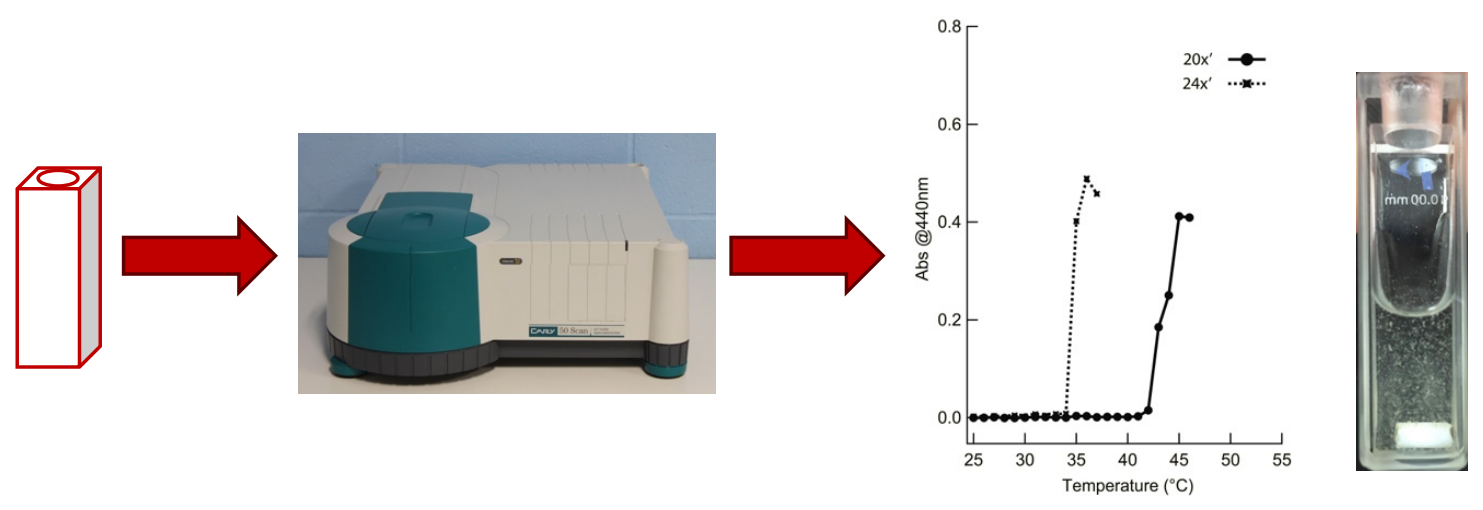

Figure 2.4 Schematic of the coacervation experiment for $20 \mathrm{x}^{\prime}$ and $24 \mathrm{x}^{\prime}$. 


\subsection{Results and Discussion}

Hydrodynamic radii can be obtained using various biophysical technique such as dynamic light scattering, analytical ultracentrifugation (AUC) and pulsed field gradient NMR (PFG NMR). PFG NMR was used to calculate hydrodynamic radii and the values were confirmed by AUC. Hydrodynamic properties are good indicators of secondary and tertiary structure of proteins. Folded proteins typically have smaller hydrodynamic radii than disordered proteins. However, a decrease in hydrodynamic radius observed in IDPs does not necessarily indicate formation of secondary structure. Aggregation or oligomerization leads to an increase in hydrodynamic radius which is not observed here.

Different scaling laws were used to estimate hydrodynamic radii $\left(\mathrm{R}_{\mathrm{H}}\right)$ of folded, denatured and intrinsically disordered proteins (IDP) as shown in the following equations $^{58}$ :

$$
\begin{gathered}
R_{H}^{\text {folded }}=4.92 N^{0.285} \\
R_{H}^{\text {denatured }}=2.33 N^{0.549} \\
R_{H}^{I D P}=2.49 N^{0.509} \\
R_{H}{ }^{M F K}=\left(1.24\left(f_{P R O}\right)+0.904\right)(0.00759|Q|+0.963) * 2.49 N^{0.509}
\end{gathered}
$$

Equation 2.6 for IDPs was optimized by Marsh and Forman-Kay to account for the effects of proline content and net charge has a reported RMSD value of $2.37 \AA$.

The experimentally obtained hydrodynamic radii of the minielastins were found to be lower than the calculated $R_{H}$ for IDPs and, as expected, significantly higher than the $R_{H}$ of a folded protein of the same number of residues. These also indicated that the constructs were more compact than typical IDPs and less structured than a folded protein. 


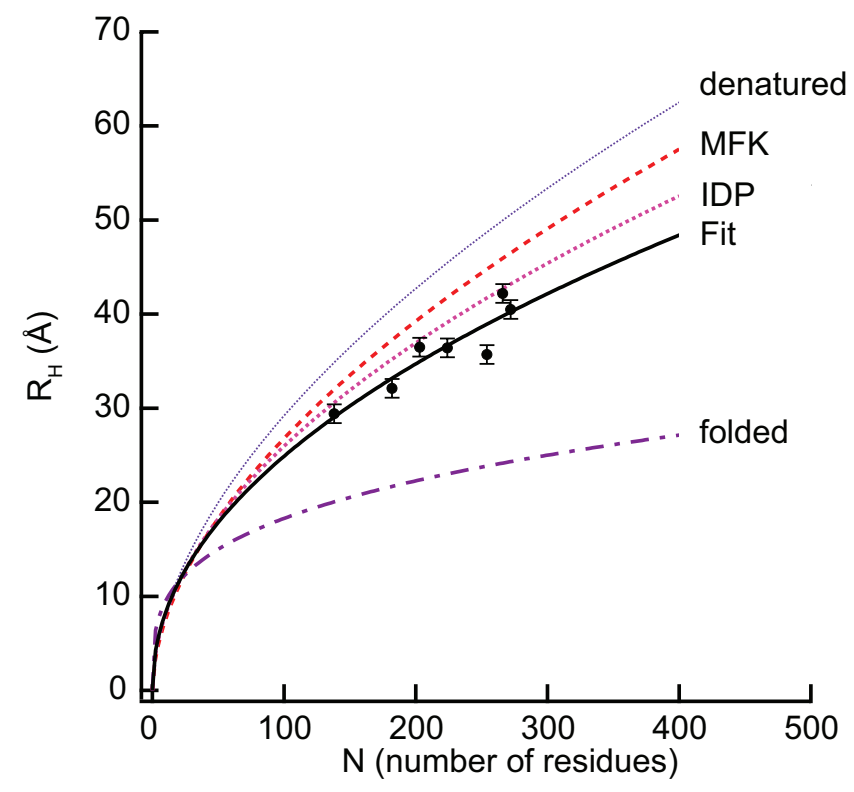

Figure $2.5 \mathrm{Hydrodynamic} \mathrm{radii} \mathrm{of} \mathrm{minielastins} \mathrm{at} 25^{\circ} \mathrm{C}$ (filled circles) with calculated $R_{\mathrm{H}}$ using scaling laws, eqs. 2.3-2.6.

Minielastins, on average, have $13 \%$ proline content, which according to eq. 2.6 contributes to a $17 \%$ increase in hydrodynamic radii compared to IDPs. Although it is expected that prolines increase the hydrodynamic radii of minielastins because of its propensity to form the secondary structure PPII, this is not observed here. This is likely because all prolines in these minielastin constructs are followed by the highly flexible glycine residue. Compared to the minielastins studied here, ELPs with the sequence $(\mathrm{VPGVG})_{\mathrm{n}}$ are less compact, have higher proline content $(20 \%$ vs $13 \%)$ and have no net charge at neutral $\mathrm{pH} .{ }^{90}$ Hydrodynamic radii of minielastins were fit to $\mathrm{R}_{\mathrm{H}}=\mathrm{R}_{0} \mathrm{~N}^{v}$, Figure 2.5 , to obtain the scaling exponent, $v=0.480 \pm 0.051$. This is in good agreement with $v=$ 0.509 obtained from eq. $2.6^{58}$ and the theoretical value, $v=0.5$, for a freely jointed chain. ${ }^{61}$ The pre-exponential factor $2.73 \pm 0.75 \AA$ is consistent with $2.70 \AA$ using eq. 2.6 with proline and net charge taken into account. These minielastins behave like a freely 


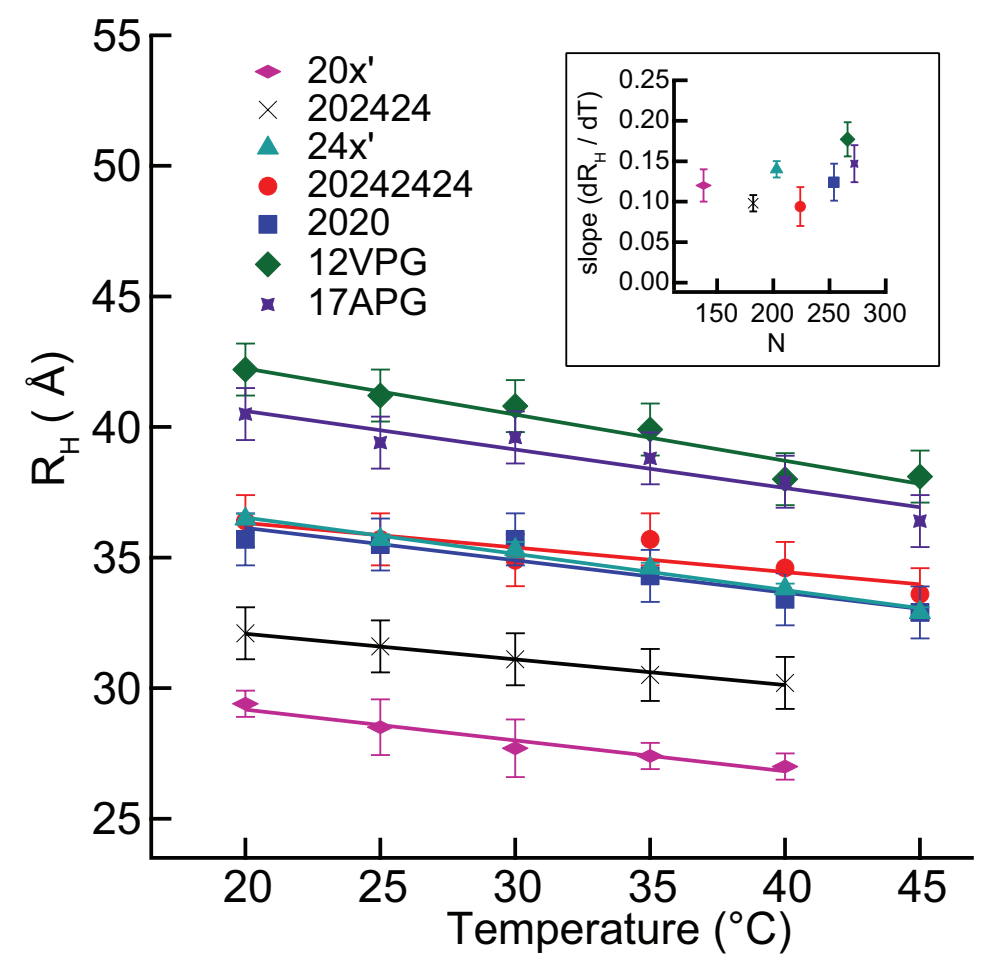

Figure 2.6 Temperature-dependence of hydrodynamic radii of minielastins. Slopes of the $\mathrm{R}_{\mathrm{H}}$ versus temperature plot are shown in the inset and reported in Table 2.1.

jointed chain with a segment length intermediate between the per residue lengths of known secondary structures i.e., $\alpha$-helix (1.5 $)$ ), $\beta$-sheet and PPII (3.1 $\AA)$.

Consistent decrease in hydrodynamic radii was observed with increasing temperature, Figure 2.6. It is surprising that proteins become more compact as the temperature increase. In the previous chapter, no change in chemical shifts were observed with increasing temperature whereas $\mathrm{CD}$ spectra revealed no apparent change in intramolecular interactions and possible disruption of PPII conformation. The temperature-dependence of $\mathrm{R}_{\mathrm{H}}$ suggests further compaction with increased temperature. In Figure 2.6, a systematic decrease in $R_{H}$ was observed for all 


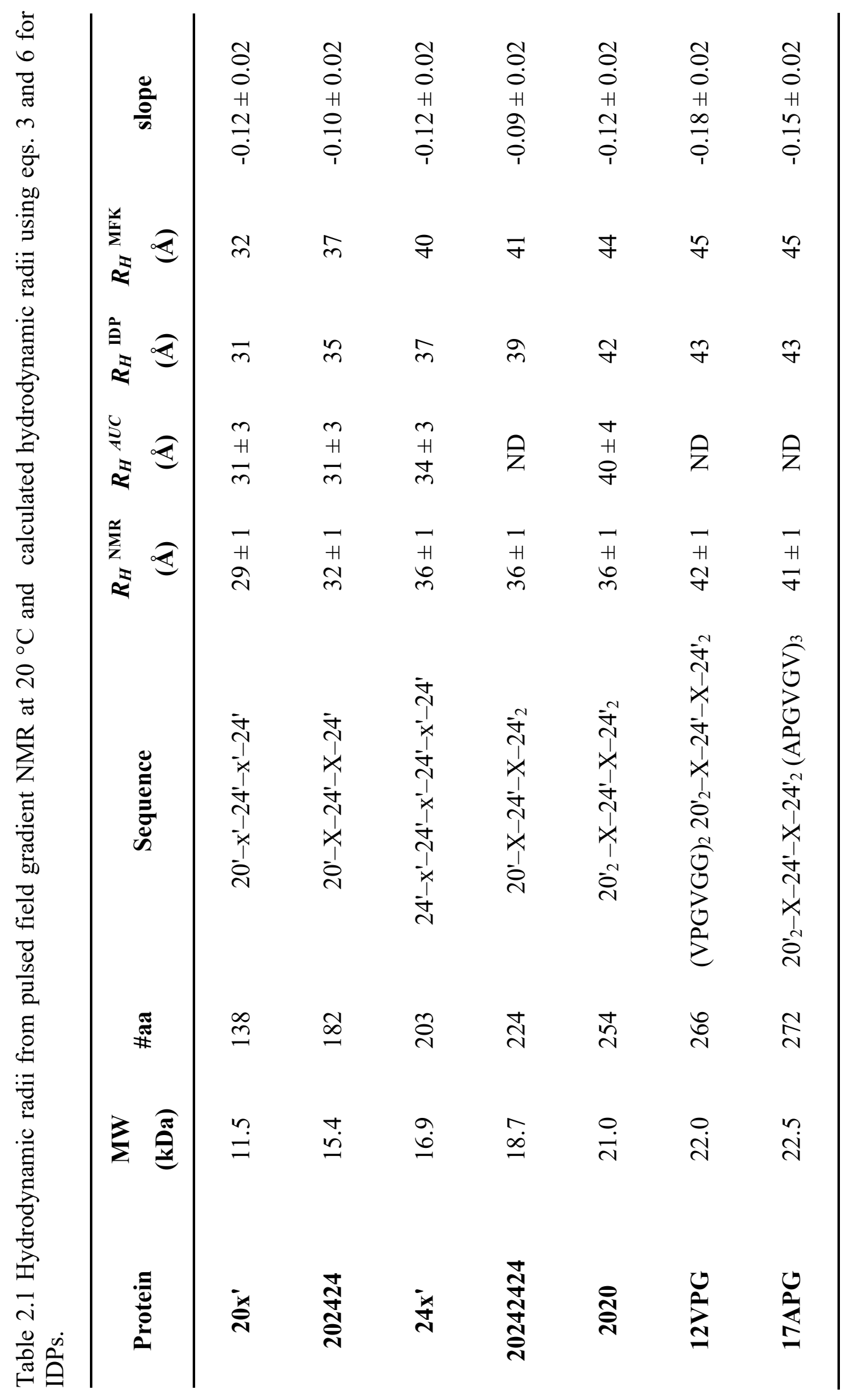


minielastin constructs with slopes ranging from -0.08 to -0.18 where the larger change was observed for higher molecular weight constructs. The same temperature-dependence was observed for intrinsically disordered region of the activator for thyroid hormone and retinoid receptors (ACTR) and the cytosolic C-terminal distal tail of human sodiumproton exchanger 1 (hNHE1cdt) that have shown a decrease in hydrodynamic radii with increase in temperature. This was attributed to the redistribution of the populations of the statistical coil. ${ }^{72}$ Similarly, for the intrinsically disordered region of p53 protein, the decrease in $R_{H}$ was explained as a result of PPII denaturation. ${ }^{73}$ In the case of minielastins, compaction can also be attributed to denaturation of PPII albeit ${ }^{13} \mathrm{C} N M R$ did not show significant change in chemical shifts due to the similarity in chemical shifts of PPII with disordered protein. ${ }^{30,91} \mathrm{MD}$ simulations of ELP by Li and coworkers have shown that increasing temperature causes the exclusion of water molecules, thus, decreases the protein-water-protein interactions. ${ }^{53}$, 92 This leads to a decrease in $\mathrm{R}_{\mathrm{H}}$ consistent with the observed temperature-dependence of $\mathrm{R}_{\mathrm{H}}$ for minielastin constructs. Although the disruption of PPII cannot be completely disregarded, CD spectra for $24 \mathrm{x}^{\prime}$ has shown no change in $220 \mathrm{~nm}$ intensity at increased temperature unlike the observed decrease of $220 \mathrm{~nm}$ intensity in ACTR and hNHE1cdt. Therefore, the observed decrease in $\mathrm{R}_{\mathrm{H}}$ can be explained by 1) redistribution of the population, 2) denaturation of PPII, and 3) exclusion of water molecules or hydrophobic collapse.

This observed temperature-dependence of the hydrodynamic radii of minielastins is important in understanding the coacervation process. It has been known that ionic strength, molecular weight and ELP concentration modulate the coacervation temperature $\left(T_{c}\right)$. Meyer and Chilkoti postulated an equation to calculate the coacervation temperature 
based on protein concentration and ELP constructs. ${ }^{93}$ As expected, designed minielastins undergo coacervation like tropoelastin and ELPs. The coacervation temperatures of the minielastin constructs are reported in Table 2.2.

Previous studies have shown that mean hydrophobicity and MW are inversely correlated to $\mathrm{T}_{\mathrm{c} .}{ }^{20,83,93}$ Increasing MW by adding more hydrophobic module decreases $\mathrm{T}_{\mathrm{c}}$ across the minielastin constructs with the same cross-linker. As expected, $\mathrm{R}_{H}$ increases with increasing MW. The decrease in $T_{c}$ is then correlated with an increase in $R_{H}$ with Pearson's coefficient $r^{2}=.905$. More interestingly, decrease in the cross-linker length and $\mathrm{R}_{\mathrm{H}}$ also decrease $\mathrm{T}_{\mathrm{c}}$ as observed in $20 \mathrm{x}^{\prime}$ and 202424 . Thus, the inverse relationship between $\mathrm{R}_{\mathrm{H}}$ and $\mathrm{T}_{\mathrm{c}}$ can only be applied to constructs with the same cross-linker.

Using constructs with varying length of hydrophobic module, the change in $\mathrm{T}_{\mathrm{c}}$ per set of six amino acid repeat (VPGVGG or APGVGV) were estimated. An example is the addition of (VPGVGG) 2 in the N-terminus of 2020 to yield the 12 VPG construct resulted to an increase of $3{ }^{\circ} \mathrm{C}$ in $\mathrm{T}_{\mathrm{c}}$. Therefore, each repeat resulted in a $1.5{ }^{\circ} \mathrm{C}$ decrease in $\mathrm{T}_{\mathrm{c}}$. The same is true for the addition of $(\mathrm{APGVGV})_{3}$ that yielded a $5{ }^{\circ} \mathrm{C}$ change in the transition temperature. Thus, each repeat result to a change of $1.7^{\circ} \mathrm{C}$. Least-square fitting using eq. 2.7 and 202424 as the reference protein resulted similar values with fit parameters

$$
T_{c}=T_{r e f}+\left(a \Delta n_{A P G V G V}+b \Delta n_{V P G V G G}+c \Delta n_{x}\right)
$$

$a=-1.5 \pm 0.2, b=-1.7 \pm 0.3$, and $c=2.9 \pm 1.4$. Calculated values are shown in Table 2.2

The signs of the coefficients indicate how the addition of each module affects the coacervation temperature. Increasing hydrophobic module length and decreasing cross- 
link module length decreases the coacervation temperature. Similar to Meyer and Chilkoti's equation ${ }^{93}$ for ELP, eq. 2.7 is specific to the minielastin sequence repeats that were studied. The calculated coefficient suggests that the decrease in transition temperature from a VPGVGG repeat is somewhat greater than from an APGVGV repeat.

Table 2.2 Calculated coacervation temperature ( $\left.T_{c}\right)$ using eq. 2.7 .

\begin{tabular}{|c|c|c|c|c|c|c|c|}
\hline Protein & \#aa & $\begin{array}{c}\mathbf{R}_{\mathbf{H}} \mathbf{N M R} \\
(\AA)\end{array}$ & $\mathbf{T}_{\mathbf{c}}\left({ }^{\circ} \mathbf{C}\right)$ & $\mathbf{n}_{\text {APGVGV }}$ & nVPGVGG & $\mathbf{n}_{\mathbf{x}}{ }^{\mathbf{a}}$ & $\begin{array}{c}\mathbf{T}_{\mathbf{c}}\left({ }^{\circ} \mathbf{C}\right) \\
\text { calc }^{b}\end{array}$ \\
\hline $20 x^{\prime}$ & 138 & $29 \pm 1$ & 44 & 14 & 5 & 2 & $45 \pm 2$ \\
\hline 202424 & 182 & $32 \pm 1$ & 52 & 14 & 5 & 4 & $50 \pm 2$ \\
\hline $24 x^{\prime}$ & 203 & $36 \pm 1$ & 36 & 28 & 0 & 3 & $35 \pm 2$ \\
\hline 20242424 & 224 & $36 \pm 1$ & 37 & 21 & 5 & 4 & $40 \pm 2$ \\
\hline 2020 & 254 & $36 \pm 1$ & 32 & 21 & 10 & 4 & $32 \pm 2$ \\
\hline $12 \mathrm{VPG}$ & 266 & $42 \pm 1$ & 29 & 21 & 12 & 4 & $28 \pm 2$ \\
\hline 17APG & 272 & $41 \pm 1$ & 27 & 24 & 10 & 4 & $27 \pm 2$ \\
\hline
\end{tabular}

${ }^{a} \mathrm{x}=\mathrm{D}(\mathrm{Q}) \mathrm{A}_{5} \mathrm{KA}_{2(3)} \mathrm{KF}(\mathrm{Y})$, sequence variations are shown in parenthesis.

${ }^{\mathrm{b}} \mathrm{T}_{\mathrm{c}}$ calc was calculated using $\Delta \mathrm{n}=\mathrm{n}_{\mathrm{i}}-\mathrm{n}_{\mathrm{ref}}$

While the difference is comparable to the indicated parameter uncertainties, this is surprising given that the coacervation temperature is affected by hydrophobicity and the Kyte-Doolittle hydropathies of APGVGV and VPGVGG are 1.3 and 0.93 , respectively (Figure 2.7). A similar trend was observed by Miao and coworkers. ${ }^{12}$ Specifically, 


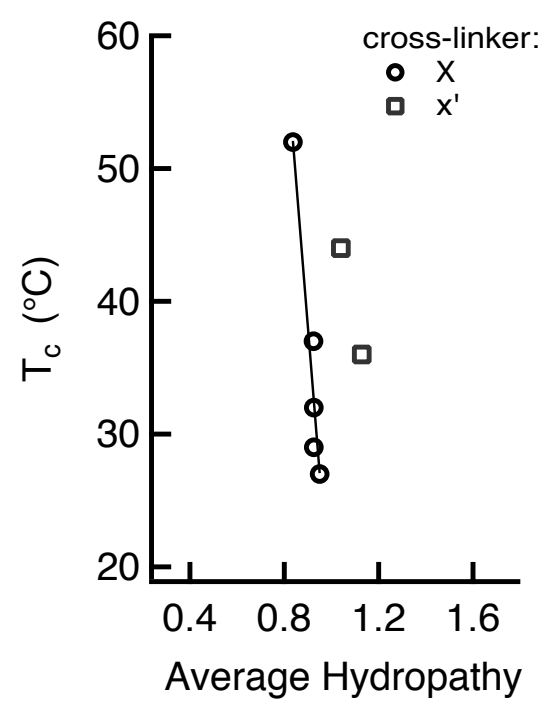

Figure 2.7 Dependence of coacervation temperature with the average per residue hydrophobicity of minielastin constructs using Kyte-Doolittle hydropathy indices.

replacing a module rich in VPGVGG repeats with a longer module rich in APGVGV repeat was observed to coacervate at a higher temperature. Factors other than the hydrophobicity affect the transition temperature. Reversing the order of P and $\mathrm{G}$ in the sequence, i.e. $(\mathrm{VPGVG})_{\mathrm{n}}$ to $(\mathrm{VGPVG})_{\mathrm{n}}$, was observed by $\mathrm{Li}$ and coworkers to increase the coacervation temperature, altered the $\mathrm{CD}$ spectrum and increased the radius of gyration, $\mathrm{R}_{\mathrm{G}}{ }^{94}$ Thus, changing the order of the residues which does not change the hydrophobicity also lowers the coacervation temperature. It is possible that the smallest residue (glycine) after proline increases flexibility i.e. the configurational entropy and this contributes to a decrease in the coacervation temperature. Similarly, VPGVGG which is less hydrophobic but more flexible than APGVGV has a greater effect in lowering the coacervation temperature. Thus, both hydrophobicity and high conformational disorder contribute to coacervation process. 
In addition to temperature, pressure also perturbs protein structure. Pressure increase favors the conformation with lower molar volume. Therefore, pressure-induced denaturation suggests that unfolded proteins have lower molar volume than their natively folded conformation. This is attributed to higher inherent internal void volume of folded proteins. ${ }^{67,69,95-96}$ High-pressure NMR provides an atomistic insight of pressure effects on protein unfolding and allows detection of low lying excited states. ${ }^{97-98}$ The combination of temperature and pressure effects on chemical shifts have revealed important changes in protein conformation. ${ }^{99}$ Temperature-dependence of amide chemical shifts for minielastins, as discussed in Chapter 1, were consistent with amide protons hydrogen bonded to water. Negative coefficients have been attributed to elongation of the hydrogen bonds and positive coefficients are characteristic of shortening of these hydrogen bonds. ${ }^{100}$ While changes in chemical shifts with temperature were shown to be linear unlike the changes observed with pressure that are nonlinear and follow the relation ${ }^{98}$ :

$$
\delta(p p m)=\delta_{0}+B_{1}\left(P-P_{0}\right)+B_{2}\left(P-P_{0}\right)^{2}
$$

where $\delta$ and $\delta_{0}$ are chemical shifts in ppm at pressure $\mathrm{P}$ and $\mathrm{P}_{0}(1$ bar $) . \mathrm{B}_{1}$ and $\mathrm{B}_{2}$ are the first and second order coefficients. Koehler and coworkers have reported pressuredependent coefficients obtained by least square fitting of chemical shifts versus pressure for the model tetrapeptide Ac-GGXA- $\mathrm{NH}_{2}$ for the 20 amino acids, $\mathrm{X}$. Reported first and second order coefficients of amide protons for the model peptides have positive and negative signs, respectively. ${ }^{101-103}$ Experimental values, reported in Figure 2.8 and Table 2.3, were comparable in magnitude to the reported random coil coefficients. 

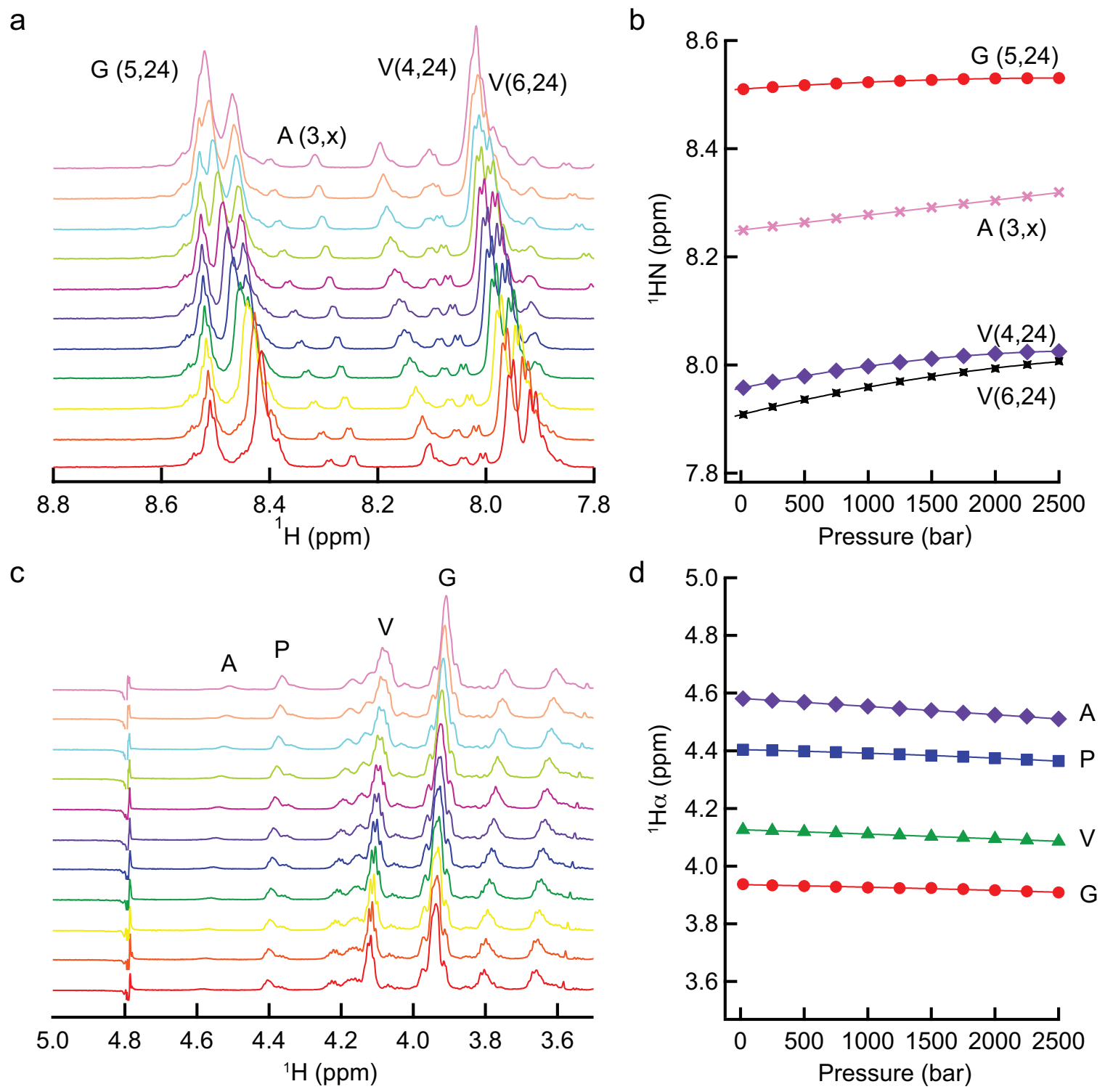

Figure 2.8 Pressure-dependence of (a-b) $\mathrm{HN}$ and (c-d) $\mathrm{H} \alpha$ chemical shifts of $24 \mathrm{x}^{\prime}$ measured at 20 to 2500 bar with second order coefficients reported in Table 2.3.

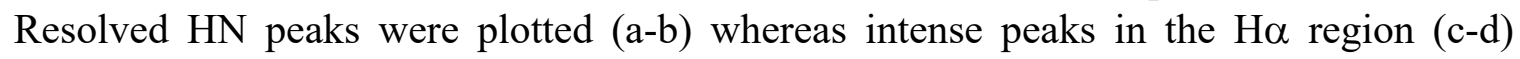
correspond to the hydrophobic repeat residues that are abundant in this minielastin construct. 
As shown in Figure 2.8a and b, the increase in pressure causes a downfield shift in amide proton chemical shifts. This observed trend is consistent with shortening of hydrogen bonds. $\mathrm{B}_{2}$, the coefficient of the quadratic term is comparable to the model peptides. In contrast, $\mathrm{H} \alpha$ chemical shifts moved upfield with an increase in pressure, Figure $2.8 \mathrm{c}$ and $\mathrm{d} . \mathrm{B}_{2}$ values are eight times higher than that mean value reported by Erlach and coworkers. ${ }^{102}$ Changes in secondary structure will move $\mathrm{HN}$ and $\mathrm{H} \alpha$ towards the same direction. Thus, the observed pressure-induced changes in $\mathrm{HN}$ and $\mathrm{H} \alpha$ chemical shifts are not consistent with changes in secondary structure. Recall that $\mathrm{HN}$ and $\mathrm{H} \alpha$ chemical shifts move downfield with disruption of $\alpha$-helices and the opposite is observed for the disruption of $\beta$-sheet structure. ${ }^{17,19}$ Changes in secondary structure such as enhancement of $\alpha$-helix will result in an upfield shift of $\mathrm{HN}$ and $\mathrm{H} \alpha .{ }^{102}$ For example,

Table 2.3 Coefficients of second order polynomial fitting of proton chemical shifts versus pressure.

\begin{tabular}{cccc}
\hline nuclei & $\boldsymbol{\delta}(\mathbf{p p m})$ & $\mathbf{B}_{\mathbf{1}}\left(\times \mathbf{1 0} \mathbf{0}^{-\mathbf{5}} \mathbf{p p m} / \mathbf{b a r}\right)$ & $\mathbf{B}_{\mathbf{2}}\left(\times \mathbf{1 0}^{-\mathbf{9}} \mathbf{p p m} / \mathbf{b a r} \mathbf{2}^{\mathbf{2}}\right)$ \\
\hline $\mathrm{HN} \mathrm{A}(3, \mathrm{x})$ & $8.249 \pm 0.001$ & $2.84 \pm 0.11$ & $-0.26 \pm 0.39$ \\
$\mathrm{HN} \mathrm{V}(4,24)$ & $7.957 \pm 0.001$ & $4.97 \pm 0.04$ & $-8.92 \pm 0.16$ \\
$\mathrm{HN} \mathrm{G}(5,24)$ & $8.510 \pm 0.001$ & $1.63 \pm 0.02$ & $-3.22 \pm 0.08$ \\
$\mathrm{HN} \mathrm{V}(6,24)$ & $7.908 \pm 0.001$ & $5.89 \pm 0.10$ & $-7.86 \pm 0.38$ \\
$\mathrm{H} \alpha \mathrm{A}$ & $4.582 \pm 0.001$ & $-2.73 \pm 0.13$ & $-0.44 \pm 0.48$ \\
$\mathrm{H} \alpha \mathrm{P}$ & $4.404 \pm 0.001$ & $-1.02 \pm 0.04$ & $-2.31 \pm 0.16$ \\
$\mathrm{H} \alpha \mathrm{G}$ & $3.936 \pm 0.001$ & $-0.79 \pm 0.21$ & $-1.07 \pm 0.80$ \\
$\mathrm{H} \alpha \mathrm{V}$ & $4.126 \pm 0.001$ & $-1.47 \pm 0.02$ & $-5.65 \pm 0.08$ \\
\hline
\end{tabular}


observed upfield chemical shifts for both $\alpha$-helices and $\beta$-sheet structures of lysozyme were consistent with the shortening of hydrogen bonds measured via x-ray diffraction. ${ }^{104-}$ 105 Downfield movement of HN chemical shifts was also reported by Roche and coworkers for the disordered protein, $\alpha$-synuclein.$^{95}$ The chemical shift changes observed for the backbone nuclei and changes in ${ }^{3} J_{\mathrm{HN}-\mathrm{H} \alpha}$ were attributed to population change from PPII- $\beta$ to PPII conformations. ${ }^{95}$ In a more recent study by Ramanujam $e t$ al., these changes were accompanied by a $3 \%$ decrease in hydrodynamic radius. ${ }^{106}$

Hydrodynamic radii of natively folded proteins are smaller than IDPs and minielastins, Figure 2.5. Thus, pressure-induced unfolding given that the volume of the unfolded state is lower than the natively folded state will result in an increase in $R_{H}$. The volume change with pressure is determined by the balance between the void volume and the hydration volume. ${ }^{71}$ Unfolding of globular proteins will increase the Stokes volume $\left(4 / 3 \pi \mathrm{R}_{\mathrm{H}}^{3}\right)$ but not necessarily the molar volume of the protein because of their different shapes. Recall that the AUC frictional ratio, $f / f_{0} \sim 2$, found in disordered proteins such as minielastins and tropoelastin shows that they are well hydrated and/or asymmetric compared to globular proteins. ${ }^{30,75}$ High pressure PFG NMR experiments of minielastin constructs have consistently shown a decrease in hydrodynamic radii, similar to what was observed for $\alpha$-synuclein. Figure 2.9 shows that minielastin constructs at high pressure undergo an $\sim 10 \%$ decrease in $\mathrm{R}_{\mathrm{H}}$ from 20 to 2500 bar. The pressure effect on $\mathrm{R}_{\mathrm{H}}$ can be explained by changes in population of unfolded states similar to $\alpha$-synuclein, ${ }^{106}$ a decrease in void volume ${ }^{107}$, or compression of hydrogen bonds. ${ }^{108}$ The observed decrease in $\mathrm{R}_{\mathrm{H}}$ suggests that the partial $\alpha$-helices in the cross-link module are less likely to unfold within the range of pressure used. ${ }^{109}$ Similarly, a small decrease in $\mathrm{R}_{\mathrm{H}}$ observed for 
denatured lysozyme was attributed to the compaction of hydrogen bonds and expansion of other parts of the protein. ${ }^{104}$

The decrease in hydrodynamic radii of minielastin constructs with increased temperature and pressure had been observed using PFG NMR. This suggests that at higher temperature, where coacervation occurs, the more compact states are favored. This also shows that coacervation is an endothermic process (favored at higher temperature) analogous to the spontaneous recoil of elastin fibers that were found to have positive heat libration and entropy values. ${ }^{1,110}$ The degree of compaction was found to be greater with temperature $\left(\sim 0.13 \AA /{ }^{\circ} \mathrm{C}\right)$ than pressure $(\sim 0.002 \AA /$ bar $)$ as the increase in kinetic energy allows the access of different conformations given that IDPs has a rather flat free energy landscape.

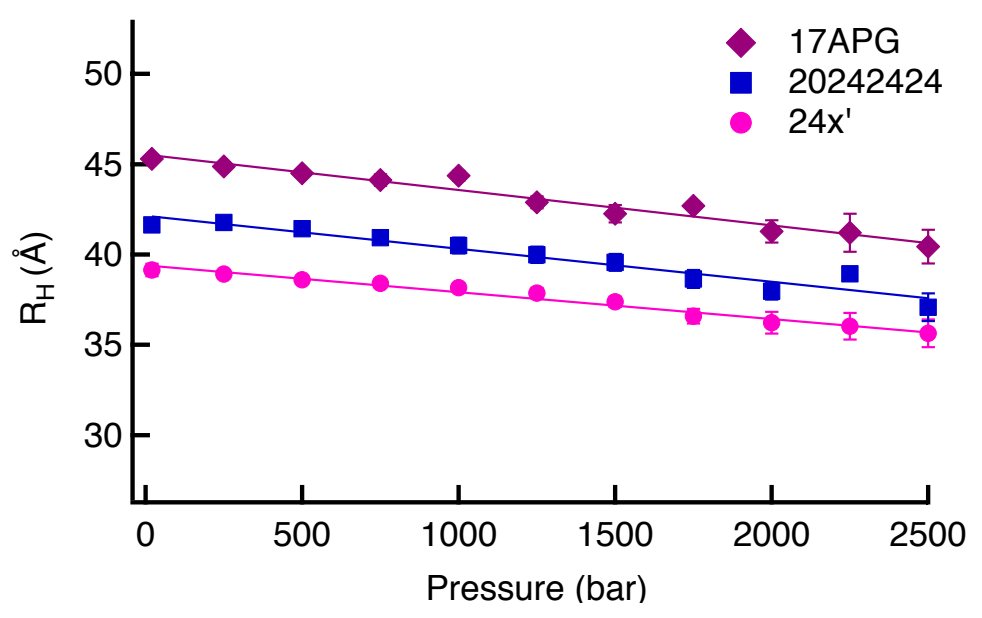

Figure 2.9 Change in hydrodynamic radii of 17APG, 20242424 and $24 x^{\prime}$ constructs with pressure ranges from 20 to 2500 bar.

In this chapter, the change in coacervation temperature associated with increase in hydrophobic and cross-link module was explored. Further studies on hydrodynamic radii with varying temperature and pressure is important to draw the phase diagram of the 
coacervation process of minielastins. Two- and three-dimensional NMR experiments with temperature and pressure perturbations will provide an atomistic picture of the changes associated with coacervation in terms of hydration and conformational states of minielastins. Recently, the effect of temperature and pressure on liquid-liquid phase transition of soluble $\alpha$-elastin was investigated by Cinar and coworkers. ${ }^{107}$ Using FTIR, CD and UV-vis measurements, they have shown that increase in pressure resulted to a more compact coacervates with less void volume in between protein solutes. Thus, understanding the temperature and pressure effect on hydration and conformational states of minielastin constructs relative to protein sequence and length is essential in modulating coacervation properties and its potential application in developing stimuli responsive elastic biomaterials. 


\section{CHAPTER 3. MINIELASTINS ARE FLEXIBLE}

\subsection{Introduction}

The structure of tropoelastin has been difficult to elucidate using structural biology techniques due to its low sequence complexity, high hydrophobicity and disordered nature. In the previous chapter, it has been established that the hydrophobic modules of minielastins are disordered whereas cross-link modules have transient $\alpha$ helical characteristics when flanked with hydrophobic modules. ${ }^{30}$ Despite the known disorder in hydrophobic domains, Baldock and coworkers using SAXS analysis showed that tropoelastin monomer adapts a canonical shape. In addition to its canonical shape, based on the cross-links between domains 10,19 and 25 observed by Mecham and coworkers $^{111}$, it has been proposed that tropoelastin follows a head-to-tail elastin assembly. The described assembly of tropoelastin introduces ordering of the monomer upon formation of the mature elastin. This has been investigated via replica exchange molecular dynamics (REMD) with explicit solvent by Tarakanova et al. Their simulations showed the existence of flexible tropoelastin with its canonical shape stabilized by salt bridges. ${ }^{112-113}$ Contrary to the head-to-tail model, more recent tandem mass spectroscopic techniques of purified mature elastin have shown the presence of multiple intradomain, interdomain and intermolecular bifunctional cross-links. ${ }^{114-115}$ This 
indicates the existence of heterogeneous cross-linking between domains of tropoelastin molecules.

Molecular dynamics simulations also show an increase in flexibility and deviations from the canonical shape of tropoelastin upon oxidative deamination of lysine residues that occurs before cross-linking. ${ }^{116}$. In addition, Pometun and coworkers have shown that mature bovine nuchal elastin has a flexible backbone carbonyl also consistent with presence of high degree of disorder. Using solid state ${ }^{13} \mathrm{C}$ NMR, the order parameter of the backbone carbonyl groups was determined to be $\mathrm{S}<0.1$ indicating a highly disordered and dynamic polypeptide. ${ }^{117}$

The degree of flexibility of minielastins were investigated using nuclear spin relaxation measurements to probe the changes that occur upon the transition from the monomer to the mature elastin matrix. NMR relaxation is a standard tool for determining dynamics of macromolecules.

\subsubsection{Nuclear spin relaxation}

Relaxation theory makes the extraction of dynamical information from NMR spin relaxation experiments possible. Perturbed nuclei in a static magnetic field undergo two types of relaxation-longitudinal (spin-lattice) and transverse (spin-spin) relaxation. Relaxation is the restoration of the thermal equilibrium, a state at which the spin population of the energy levels follows the Boltzmann distribution and the spin coherence is zero. There are two energy levels associated with a spin $1 / 2$, the $\alpha$ and $\beta$ states. The slight difference in populations between these two states results in a non-zero magnetization along the applied field, the $+\mathrm{z}$ direction. Spins aligned with the magnetic 
field are slightly in excess of spins aligned antiparallel to the magnetic field. Longitudinal relaxation $\left(R_{1}\right)$ is the rate at which the population Boltzmann distribution is reestablished.

The simplest way to measure longitudinal relaxation rate is via inversion recovery. In this experiment, a $180^{\circ}$ pulse is applied to invert the $\alpha$ and $\beta$ populations. The spins are allowed to relax for the time delay $\tau$, then a $90^{\circ}$ pulse is applied to place the magnetization in the transverse plane for detection. Increasing $\tau$ allows the magnetization to relax prior to detection as shown in Figure 3.1. To maximize NMR signals, it is generally useful to measure $R_{1}$ for every experiment so that the magnetization is in thermal equilibrium before subsequent acquisition.

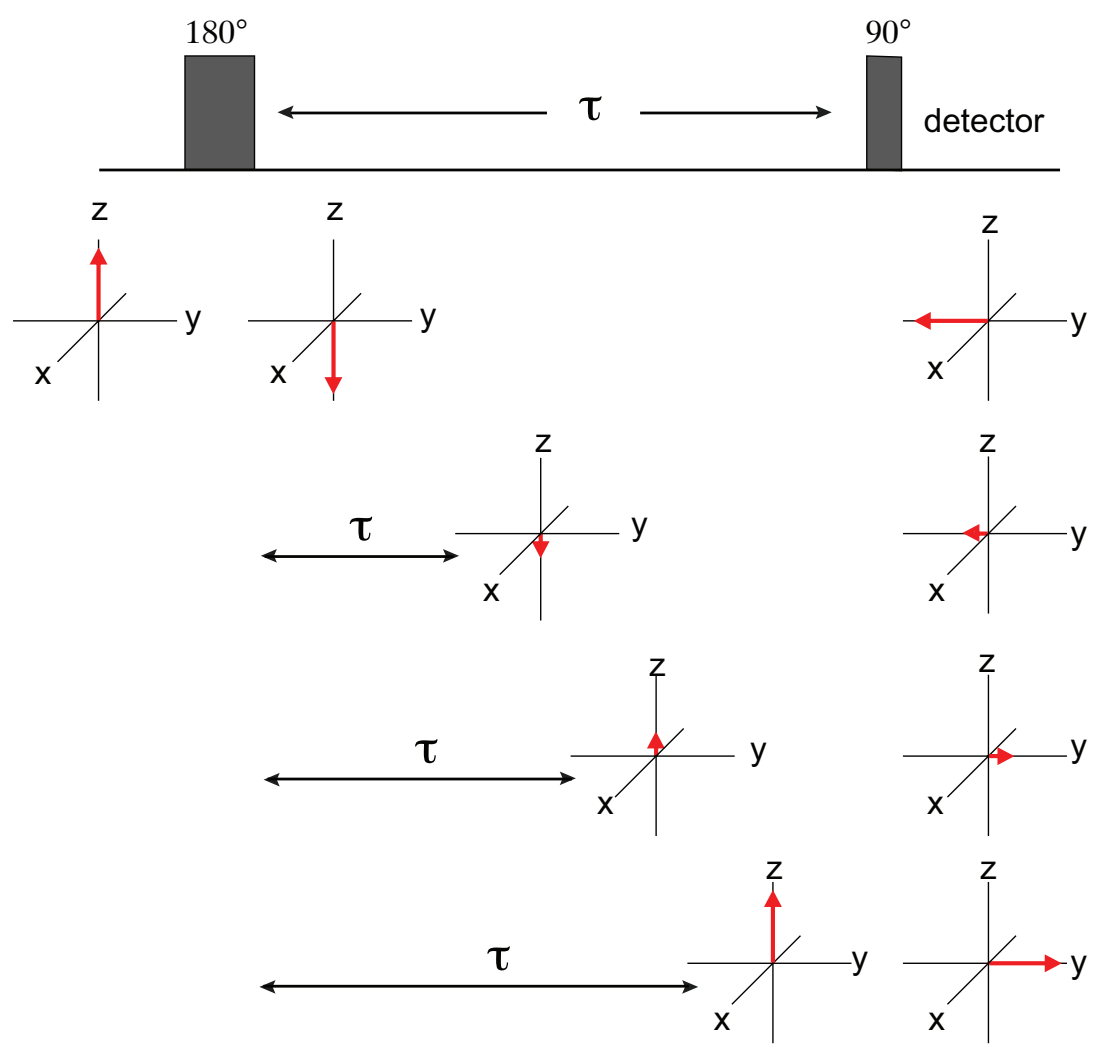

Figure 3.1 Inversion recovery pulse sequence to measure $R_{1} .180^{\circ}$ pulse inverts the $\alpha$ and $\beta$ populations. As time delay, $\tau$, increases the more time the spins have to relax back to thermal equilibrium. 
At thermal equilibrium, a net zero coherence is observed since the projection of the spin magnetization on $\mathrm{x}-\mathrm{y}$ plane is randomly arranged. When a pulse is applied, a time-dependent correlation of spin orientations in the transverse plane is created. ${ }^{118}$ Transverse relaxation $\left(R_{2}\right)$ is the rate of loss of coherence of the spins which is measured using spin echo experiment that utilizes $90^{\circ}$ and $180^{\circ}$ pulses. The first pulse takes the magnetization to the $x-y$ plane and the second pulse generates the "echo", i.e., spin evolution due to chemical shifts and magnetic field inhomogeneity is reversed but evolution due to relaxation is unaffected. As the time delay, $\tau$, increases, the spins lose coherence resulting in a decrease in magnetization due only to relaxation.

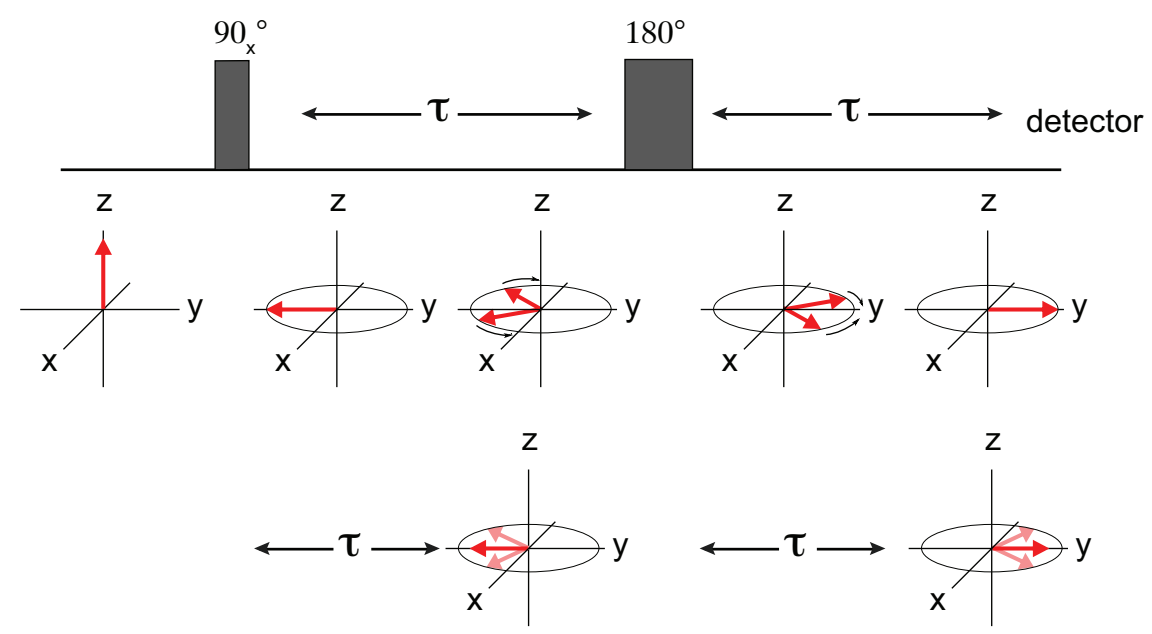

Figure 3.2 Spin echo pulse sequence to measure $R_{2}$ relaxation rate. Spin coherence is lost with increasing time delay $\tau$.

\subsubsection{Spectral Density}

Spin relaxation is due to random fluctuations in local magnetic fields caused by stochastic motions. The random local magnetic fields are due to the anisotropy of chemical shifts and dipolar couplings that are randomly reoriented by collisions with solvent molecules causing small changes in orientation via Brownian motion. For 
example, in rotational diffusion, the average time it takes for a molecule to rotate by one radian from its starting orientation is the rotational correlation time, $\tau_{c}$. When a system fluctuates spontaneously away from equilibrium, the correlation function, $\mathrm{C}(\mathrm{t})$, describes the time dependence of the return to equilibrium of the fluctuation. The simplest form of the correlation function is a single exponential with time constant or correlation time $\tau_{c}$, eq. 3.1 .

$$
\mathrm{C}(\mathrm{t})=\left\langle\mathrm{B}_{\text {loc }}{ }^{2}\right\rangle \mathrm{e}^{-\frac{|\mathrm{t}|}{\tau_{\mathrm{c}}}}
$$

Bloembergen, Pound and Purcell showed how the Fourier transform of the correlation function, eq. 3.2,

$$
J(\omega)=\int_{-\infty}^{+\infty} \mathrm{C}(\mathrm{t}) \mathrm{e}^{-\mathrm{i} \omega \tau} \mathrm{d} \tau=\frac{2}{5} \mathrm{~B} \frac{\tau_{\mathrm{c}}}{1+\left(\omega \tau_{\mathrm{c}}\right)^{2}}
$$

could be incorporated in time-dependent perturbation theory to determine the relaxation rates $R_{1}$ and $R_{2}$, eqs. 3.3 and 3.4

The spectral density measures the contribution of motions in the frequency domain. Fast reorienting $\mathrm{NH}$ bond vectors i.e. low $\tau_{c}$, yield broad spectral density from motions at high frequency. Slowly reorienting bond vectors are depicted by a narrow spectral density. At zero frequency, the spectral density $J(0)$, represents the area under the curve of the correlation function. Therefore, the magnitude of $J(0)$ is directly proportional to $\tau_{c}$.

For spin $1 / 2$, the dominant sources of relaxation are dipole-dipole interactions and chemical shift anisotropy. Dipole-dipole interactions are local magnetic field fluctuations caused by nearby nuclei. Dipole-dipole interactions are dependent on the distance of the nuclei hence, the strength of interaction decreases with $1 / \mathrm{r}^{6}$. The chemical shift depends 
on the orientation of the molecules with respect to the static magnetic field due to anisotropy of the chemical shift interaction. Since it is highly influenced by external magnetic field, the contribution from CSA increases with increasing magnetic field strength. The relaxation rates can be expressed in terms of spectral density sampled at 0 , $\omega_{N}, \omega_{H}$, and $\omega_{H} \pm \omega_{N}$ as shown in the following equations for the amide bond vector:

$$
\begin{gathered}
R_{1}=\frac{\mathrm{d}^{2}}{4}\left\{J\left(\omega_{\mathrm{H}}-\omega_{\mathrm{N}}\right)+3 J\left(\omega_{\mathrm{N}}\right)+J\left(\omega_{\mathrm{H}}+\omega_{\mathrm{N}}\right)\right\}+\mathrm{c}^{2} J\left(\omega_{\mathrm{N}}\right) \\
R_{2}=\frac{\mathrm{d}^{2}}{8}\left\{4 J(0)+J\left(\omega_{\mathrm{H}}-\omega_{\mathrm{N}}\right)+3 J\left(\omega_{\mathrm{N}}\right)+6 J\left(\omega_{\mathrm{H}}\right)+6 J\left(\omega_{\mathrm{H}}+\omega_{\mathrm{N}}\right)\right\}+ \\
\frac{\mathrm{c}^{2}}{6}\left\{4 J(0)+3 J\left(\omega_{\mathrm{N}}\right)\right\}
\end{gathered}
$$

where the dipolar coupling constant $d=\mu_{0} h \gamma_{H} \gamma_{N} / 8 \pi^{2} r_{N H}{ }^{3}, \quad \mu_{0}$ is permeability of free space, $\gamma_{\mathrm{H}}$ and $\gamma_{\mathrm{N}}$ are gyromagnetic ratios of ${ }^{1} \mathrm{H}$ and ${ }^{15} \mathrm{~N}$, respectively. The distance between the ${ }^{1} \mathrm{H}$ and ${ }^{15} \mathrm{~N}$ is $\mathrm{r}_{\mathrm{NH}}{ }^{3}=1.02 \AA$, whereas the chemical shift anisotropy constant $c=\Delta \sigma \omega_{N} / \sqrt{3}, \Delta \sigma=-172 \mathrm{ppm}$ is the CSA for axially symmetric amide and $\omega_{N}$ is the Larmor frequency of ${ }^{15} \mathrm{~N}$.

Spin-lattice relaxation rates of ${ }^{1} \mathrm{H}$ and ${ }^{15} \mathrm{~N}$ together determine the steady-state nuclear Overhauser enhancement, NOE. In this experiment, ${ }^{1} \mathrm{H}$ nuclei are irradiated by a strong RF field so the ${ }^{1} \mathrm{H}$ spin state populations are equalized and during irradiation cross-relaxation with ${ }^{15} \mathrm{~N}$ contributes to a change in the ${ }^{15} \mathrm{~N}$ signal intensity. The ratio of the signals with and without irradiation is the NOE. In the case of ${ }^{15} \mathrm{~N}$ with a negative gyromagnetic ratio, the NOE is less than 1 and depends on the spectral density as shown below, eq. 3.5. 


$$
\mathrm{NOE}=1+\frac{\mathrm{d}^{2} \gamma_{\mathrm{H}}}{4 \mathrm{R}_{1} \gamma_{\mathrm{N}}}\left\{6 J\left(\omega_{\mathrm{H}}+\omega_{\mathrm{N}}\right)-J\left(\omega_{\mathrm{H}}-\omega_{\mathrm{N}}\right)\right\}
$$

Based on eqs $3.3-3.5$, nuclear spin relaxation is a powerful tool to probe the rotational motions at picosecond to nanosecond timescale. However, a physical interpretation of the dynamics can only be obtained by setting verifiable assumptions and modeling the spectral density.

The dynamical information embedded in the relaxation data can be extracted by fitting a parameterized spectral density to the data. Ideally, the fit parameters of the spectral density are related to important dynamical properties of the protein under study and then validated against the spectral density map (SDM).

The relaxation observables, $R_{1}, R_{2}$, and NOE are linear combinations of the spectral density at various frequencies, eqs. 3.3-3.5, and can be used to calculate the spectral density at five frequencies. The spectral density at $0, \omega_{\mathrm{N}}, \omega_{\mathrm{H}}, \omega_{\mathrm{H}}+\omega_{\mathrm{N}}$ and $\omega_{\mathrm{H}}-\omega_{\mathrm{N}}$ cannot be determined given only three equations. Peng and Wagner have shown that $J$ $\left(\omega_{\mathrm{H}}\right)$ and $J\left(\omega_{\mathrm{H}} \pm \omega_{\mathrm{N}}\right)$ have very similar magnitude, much lower than $J(0)$ and $J\left(\omega_{\mathrm{N}}\right)$ and that high frequency spectral densities are insensitive and susceptible to experimental errors. ${ }^{119}$ Farrow and coworkers then used a single frequency, $J\left(0.87 \omega_{\mathrm{H}}\right)$ to describe the high frequency spectral density and resulted a reduced form of spectral density map with only three relaxation experiments necessary. ${ }^{120}$ Reduced spectral density mapping (RSDM) although convenient, does not provide any physical interpretation of the spectral density. Since no assumptions are made, RSDM is applicable to both folded and unfolded proteins and provides a rigorous test of the assumptions made in a model spectral density. 
A general form of the spectral density, eq. 3.6, has been widely used to extract the contributions of each timescale of motions from the relaxation data. The spectral density can be expressed as a sum of Lorentzian,

$$
J(\omega)=\frac{2}{5}\left(\sum_{i=1}^{k} \frac{\mathrm{A}_{\mathrm{i}} \tau_{\mathrm{i}}}{1+\omega^{2} \tau_{\mathrm{i}}^{2}}\right) \quad \text { where } \sum_{\mathrm{i}=1} \mathrm{~A}_{\mathrm{i}}=1
$$

where $\mathrm{k}$ is the number of terms contributing to the spectral density function, $A_{i}$ as the weighting coefficient for each term and $\tau_{\mathrm{i}}$ as the timescale of motions. A more elaborate form of this spectral density, Lipari-Szabo model-free approach (LS), has been commonly used as it provides more pictorial representation of the bond vector dynamics. ${ }^{121-125}$ As the name implies, no specific model of motion is used however, assumes a separation in timescale between the overall motion and internal bond vector motion. Assuming that internal motions are independent of overall motions, the total correlation function describing the amount of motions of molecules tumbling isotropically in solution with respect to time interval $\tau$ is

$$
\mathrm{C}(\tau)=\mathrm{C}_{\mathrm{O}}(\tau) \mathrm{C}_{\mathrm{I}}(\tau)
$$

where $C_{O}(\tau)$ and $C_{I}(\tau)$ are the correlation functions for overall motions and internal motions, respectively. ${ }^{125}$

LS describes the internal motions in terms of the squared of the generalized order parameter, $S^{2}$ and an effective internal correlation time, $\tau_{\mathrm{e} .} S^{2}$ is a measurement of spatial flexibility of the $\mathrm{NH}$ bond vector and has a value between 0 and 1, indicative of flexible and rigid motions, respectively, whereas the effective correlation time is the timescale of internal motions. The LS model-free approach has a general form 


$$
J(\omega)=\frac{2}{5}\left(\frac{S^{2} \tau_{\mathrm{M}}}{1+\left(\omega \tau_{\mathrm{M}}\right)^{2}}+\frac{\left(1-S^{2}\right) \tau}{1+(\omega \tau)^{2}}\right) \text { where } \frac{1}{\tau}=\frac{1}{\tau_{\mathrm{M}}}+\frac{1}{\tau_{\mathrm{e}}} \quad \text { eq. } 3.8
$$

where $\tau_{\mathrm{M}}$ is the overall rotational correlation time that can be extracted from the relaxation data or from hydrodynamic measurements. ${ }^{124,}{ }^{126}$ Accuracy of the fit parameters, $S^{2}$ and $\tau_{\mathrm{e}}$, depends on how fast the internal motions are. ${ }^{125}$ The accuracy decreases as the $S^{2}$ value decreases; thus, LS is more accurate when used in folded proteins $\left(S^{2} \sim 0.8\right)$. When LS failed to fit the relaxation measurements, Clore and coworkers $^{127}$ introduced an additional term to describe internal motions with fast and slow timescales as shown in equation below

$$
J(\omega)=\frac{2}{5}\left(\frac{\mathrm{S}^{2} \tau_{\mathrm{M}}}{1+\left(\omega \tau_{\mathrm{M}}\right)^{2}}+\frac{\left(1-\mathrm{S}_{\mathrm{f}}^{2}\right) \tau_{\mathrm{f}}^{\prime}}{1+\left(\omega \tau_{\mathrm{f}}^{\prime}\right)^{2}}+\frac{\left(\mathrm{S}_{\mathrm{f}}^{2}-\mathrm{S}^{2}\right) \tau_{\mathrm{s}}^{\prime}}{1+\left(\omega \tau_{\mathrm{s}}^{\prime}\right)^{2}}\right)
$$

where $\frac{1}{\tau_{\mathrm{s} / \mathrm{f}^{\prime}}}=\frac{1}{\tau_{\mathrm{M}}}+\frac{1}{\tau_{\mathrm{s} / \mathrm{f}}}$. The spatial restrictions of the slow and fast internal motions are described by the order parameters $S_{s}^{2}$ and $S_{f}^{2}$, respectively, where $S^{2}=S_{s}^{2} S_{f}^{2}$. In this approach, the assumption is that the overall correlation time $\tau_{M}$, is isotropic and there is a separation between the fast and slow internal correlation times. Moreover, for systems that are highly flexible and have the slowest correlation time that cannot be described by a constant $\tau_{M}$ value as in IDPs, a distribution of the correlation time can be used. ${ }^{128-130}$ Buevich et al. ${ }^{131}$ applied the Cole-Cole distribution adopted from the analysis of polymers to the model-free approach to probe the dynamics of natively unfolded propeptide of subtilisin. Lorentzian distribution of the correlation time proposed by Ochsenbein and coworkers ${ }^{130}$ presented a more appropriate representation of the diverse motional conformation of disordered proteins. When compared with the model-free approach, $\tau_{\mathrm{o}}$, the center of the distribution is similar to $\tau_{\mathrm{s}}$ values and the width of the 
distribution, $\Delta$, evolves with the $S_{s}{ }^{2}$ suggesting heterogeneity of motions. A similar distribution was employed by Modig and Poulsen ${ }^{132}$ on the denatured acyl-coenzyme A binding protein. Time-dependent molecular dynamics trajectories application to temperature-dependent relaxation measurements were explored by Salvi and coworkers to probe the IDP-water interactions and segmental motions dependence on solvent. ${ }^{128,133}$ In this chapter, a qualitative and quantitative analysis of dynamics of minielastin, $24 x^{\prime}$, was obtained using nuclear spin relaxation. Various forms of the spectral density function were used to obtain the best fit relaxation parameters that describes the dynamics of $24 x^{\prime}$. The resulting spectral density from the fit parameters were compared with the reduced spectral density map values. The application of distribution on the slowest correlation time obtained using PFG NMR diffusion measurements were explored. The analysis provided insights on the dynamics of minielastin in solution in comparison with the order parameter of insoluble elastin fiber. Evidence that the local backbone disorder of the monomer is conserved in the mature elastin is presented. The amplitude and timescale of motions contributing to the relaxation rates are consistent with the presence or lack thereof of secondary structure. 


\subsection{Materials and Methods}

\subsubsection{Sample preparation}

${ }^{15} \mathrm{~N}$-labelled samples of minielastin construct, $24 \mathrm{x}^{\prime}$, with sequence $24^{\prime}-\mathrm{x}^{\prime}-24^{\prime}-\mathrm{x}^{\prime}-$ $24^{\prime}-\mathrm{x}^{\prime}-24^{\prime}$, where $24^{\prime}=(\mathrm{APGVGV})_{7}$, and $\mathrm{x}^{\prime}=\mathrm{DA}_{5} \mathrm{KA} \mathrm{A}_{2} \mathrm{KF}$, were expressed and prepared as described in the chapter 1 and in Greenland et al. ${ }^{30}$ All protein samples used in the relaxation experiments have concentrations $\sim 300 \mu \mathrm{M}$ at $\mathrm{pH} 6,90 \% \mathrm{H}_{2} \mathrm{O} / 10 \% \mathrm{D}_{2} \mathrm{O}$.

\subsubsection{NMR experiments}

Relaxation measurements were obtained using Bruker Avance 500, 600 and 800 $\mathrm{MHz}$ instruments equipped with cryogenically cooled probe. $R_{1}$ measurements were recorded at ten time points, between 10 to $1200 \mathrm{~ms}$ with $1 \mathrm{~s}$ recycle delay whereas $R_{2}$ measurements were recorded at eight time points between 10 to $350 \mathrm{~ms}$ with $1 \mathrm{~s}$ recycle delay. $\left[{ }^{1} \mathrm{H}\right]-{ }^{15} \mathrm{~N}$ heteronuclear NOE spectra were obtained using Bruker pulse sequence hsqcnoef3gpsi with $10 \mathrm{~s}$ saturation period and recycle delay for the $500 \mathrm{MHz}$ and 800 MHz. NMR spectra from the 500 and $800 \mathrm{MHz}$ were obtained with the assistance of Jonathan Preston and Dr. James Aramini of The City College of New York and were processed using NMRPipe and TopSpin 3.5pI7 software.

For the $600 \mathrm{MHz}$ spectrometer, chemical exchange saturation transfer (CEST) and steady-state $\left[{ }^{1} \mathrm{H}\right]-{ }^{15} \mathrm{~N}$ heteronuclear NOE measurements were recorded and analyzed by Dr. T. Michael Sabo of University of Louisville, School of Medicine. $R_{1}$ and $R_{2}$ was calculated utilizing an in-house python script as described in literature. ${ }^{134-136}$ 


\subsubsection{Relaxation Rate Analysis}

Relaxation rates were analyzed by plotting the peak intensities at time delay $\tau$ via

Igor Pro software that uses Levenberg-Marquardt algorithm for data fitting. The data was fitted using a single exponential equation to obtain residue specific relaxation rates:

$$
I(\tau)=I_{0} e^{-\tau R_{1,2}}
$$

Figure $3 \mathrm{a}$ and $\mathrm{b}$ shows the plot of intensity versus time obtained for residues $\mathrm{V}\left(6,24^{\prime}\right)$ of the hydrophobic module and $\mathrm{A}\left(5, \mathrm{x}^{\prime}\right)$ of the cross-link module. Figure $3 \mathrm{c}$ shows the superposition of the steady state NOE of proton saturated and unsaturated spectra. The
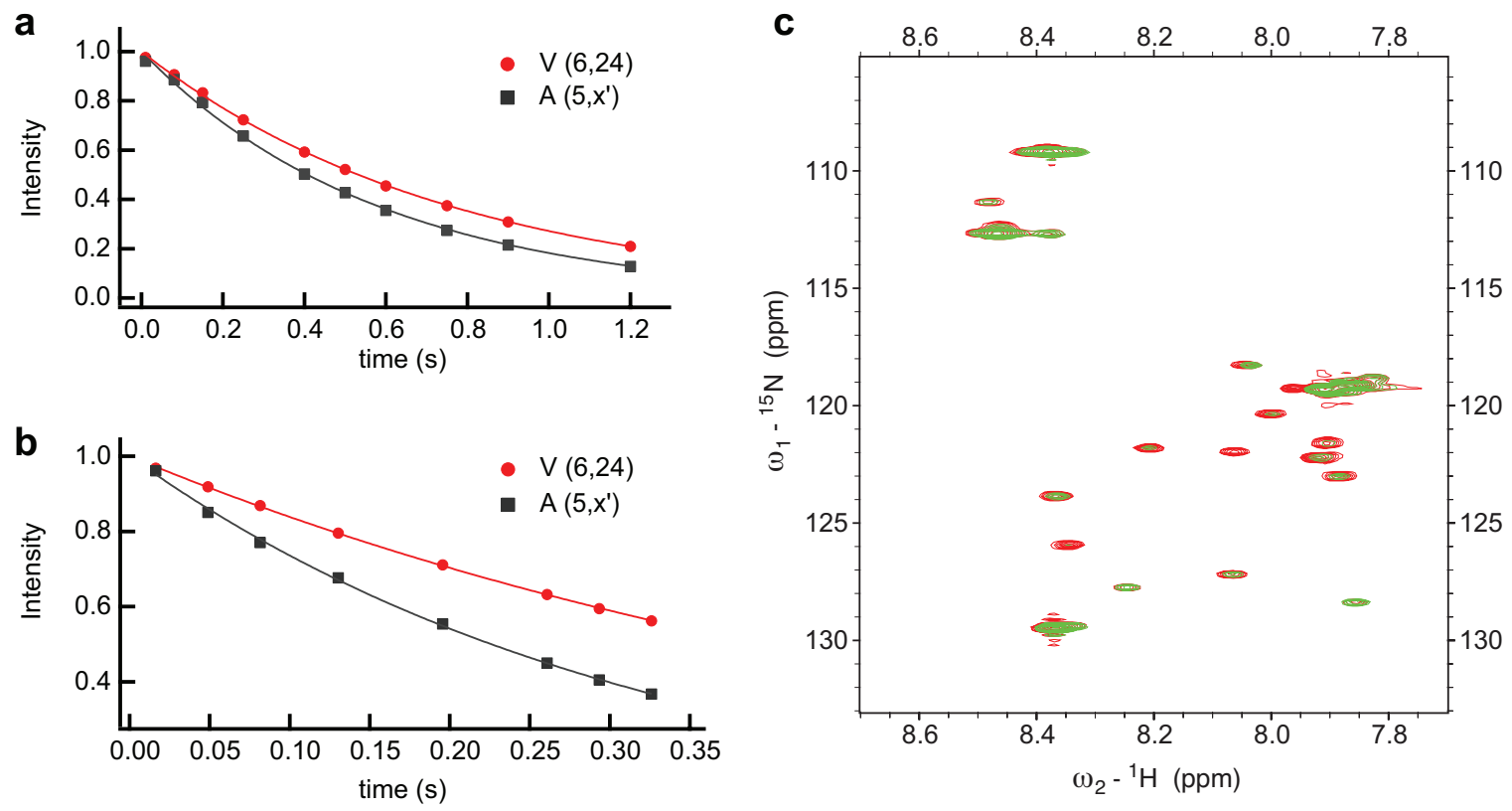

Figure 3.3 Nuclear spin relaxation observables (a) longitudinal relaxation rate $R_{1}$ (b) transverse relaxation rate $R_{2}$ of residues $\mathrm{V}(6,24)$ in red and $\mathrm{A}\left(5, \mathrm{x}^{\prime}\right)$ in gray and $(\mathrm{c})\left[{ }^{1} \mathrm{H}\right]$ ${ }^{15} \mathrm{~N}$ heteronuclear steady state NOE of $24 \mathrm{x}^{\prime}$ with and without proton saturation in green and red spectrum, respectively. 
NOE values are calculated as the ratio of peak intensities of spectra with and without ${ }^{1} \mathrm{H}$ saturation, eq. 3.11

$$
N O E=\frac{I_{\text {sat }}}{I_{\text {unsat }}}
$$

Uncertainties were obtained from the fitting of the relaxation rates whereas the uncertainties from the NOE experiments were calculated from the signal to noise. A random error of $10 \%$ from the experiment and analysis were used in the spectral density calculations.

\subsubsection{Spectral Density Mapping and Modeling}

Reduced spectral density mapping of the $24 x^{\prime}$ relaxation data were performed as described by Farrow et al. ${ }^{137}$ specifically Method 3 as proposed by Kaderavek et al. ${ }^{138}$ Experimentally determined relaxation data $R_{1}, R_{2}$, and $\left[{ }^{1} \mathrm{H}\right]-{ }^{15} \mathrm{~N}$ NOE from three spectrometers were used to calculate spectral densities at thirteen frequencies, $J(0), J$ $\left(\omega_{\mathrm{N}}\right), J\left(0.870 \omega_{\mathrm{H}}\right), J\left(0.921 \omega_{\mathrm{H}}\right)$, and $J\left(0.955 \omega_{\mathrm{H}}\right)$ at 500,600 and $800 \mathrm{MHz}$ to map the correlation function as shown in the following equations:

$$
\begin{gathered}
\mathrm{NOE}=1+\frac{\mathrm{d}^{2}}{4} \frac{\gamma_{\mathrm{H}}}{\gamma_{\mathrm{N}}}\left[5 J\left(0.870 \omega_{\mathrm{H}}\right)\right] R_{1} \\
R_{1}=\frac{\mathrm{d}^{2}}{4}\left[3 J\left(\omega_{\mathrm{N}}\right)+7 J\left(0.921 \omega_{\mathrm{H}}\right)\right]+\mathrm{c}^{2} J\left(\omega_{\mathrm{N}}\right) \\
R_{2}=\frac{\mathrm{d}^{2}}{8}\left[4 J(0)+3 J\left(\omega_{\mathrm{N}}\right)+13 J\left(0.955 \omega_{\mathrm{H}}\right)\right]+\frac{\mathrm{c}^{2}}{6}\left[3 J\left(\omega_{\mathrm{N}}\right)+4 J(0)\right]
\end{gathered}
$$

Rearranging the eqs. 3.12-3.14 yields the following expressions for the spectral density at five frequencies: 


$$
\begin{array}{cc}
J\left(0.870 \omega_{\mathrm{H}}\right)=R_{1}(\mathrm{NOE}-1) \frac{4}{5 \mathrm{~d}^{2}} \cdot \frac{\gamma_{\mathrm{N}}}{\gamma_{\mathrm{H}}} & \text { eq. } 3.15 \mathrm{a} \\
J\left(\varepsilon \omega_{\mathrm{H}}\right)=J\left(0.870 \omega_{\mathrm{H}}\right)+(\varepsilon-0.870) \omega_{\mathrm{H}} J^{\prime}\left(0.870 \omega_{\mathrm{H}}\right) & \text { eq. } 3.15 \mathrm{~b} \\
J\left(\omega_{\mathrm{N}}\right)=\frac{R_{1}-\frac{7 \mathrm{~d}^{2}}{4} J\left(0.921 \omega_{\mathrm{H}}\right)}{\frac{3 \mathrm{~d}^{2}}{4}+\mathrm{c}^{2}} & \text { eq. } 3.15 \mathrm{c} \\
J(0)=\frac{\left[\frac{R_{1}}{2}-\frac{\mathrm{d}^{2}}{8}\left[7 J\left(0.921 \omega_{\mathrm{H}}\right)+13 J\left(0.955 \omega_{\mathrm{H}}\right)\right]\right]}{R_{2}+4 \mathrm{c}^{2}} & \text { eq. } 3.15 \mathrm{~d}
\end{array}
$$

In the above eq. $3.15 \mathrm{~b}, \varepsilon=0.921$ and 0.955 and $J^{\prime}\left(0.870 \omega_{\mathrm{H}}\right)=\left[J\left(0.870 \omega_{\mathrm{H}}^{800}\right)-J\right.$ $\left.\left(0.870 \omega_{\mathrm{H}}^{500}\right)\right] /\left[0.870\left(\omega_{\mathrm{H}}^{800}-\omega_{\mathrm{H}}^{500}\right)\right.$. This approach allows the direct determination of spectral densities in four frequency and an average $J(0)$ from the relaxation data at multiple magnetic fields.

The Lipari-Szabo model-free approach, eq. 3.8, and extended Lipari-Szabo, eq. 3.9 were used in conjunction with eq. 3.6. The approaches used in this study are summarized in Table 3.1. The overall correlation time, $\tau_{\mathrm{M}}$ was estimated from hydrodynamic radius $^{30}$ as described by Yao et al. ${ }^{126}$ Gaussian distribution of hydrodynamic radii of disordered proteins was applied with full width at half height (FWHH) of $\sim 10 \AA$ shown in eq. 3.16

$$
P_{j}^{e q}=N e^{-\left(R_{H, j}-\left\langle R_{H}\right\rangle\right)^{2} / 2 \sigma^{2}}, \text { where } F W H H=2 \sqrt{2 \ln 2} \sigma, \quad \text { eq. } 3.16
$$


given that $\left\langle R_{H}\right\rangle=k_{B} T / 6 \pi \eta D_{t}$ from PFG NMR translational diffusion, were used to calculate the average overall rotational correlation time, eq. 3.17.

$$
\tau_{M, j}=\frac{1}{6 D_{r}}=\frac{4 \pi \eta R_{H, j}{ }^{3}}{3 k_{B} T} \text { and that }\left\langle\tau_{M}\right\rangle=\sum_{j=1}^{L} P_{j}^{e q} \tau_{M, j}
$$

$k_{B}$ is the Boltzmann constant, $\eta$ is the solvent viscosity and $\mathrm{L}=18$, therefore, the correlation function for the overall motion is given as,

$$
\mathrm{C}_{\mathrm{O}}(\mathrm{t})=\frac{1}{5} \sum_{j=1}^{L} P_{j}^{e q} e^{-\frac{t}{\tau_{M, j}}}
$$

Finally, with Gaussian distributed $\tau_{M}$ in the range at which $\tau_{M}$ is much greater than the inverse of the lowest frequency detected $\left(\omega_{\mathrm{N}}, 500^{-1} \sim 0.3 \mathrm{~ns}\right)$ and $\tau_{\mathrm{M}}>>\tau_{\mathrm{s} / \mathrm{f}}$, the spectral density takes the form

$$
J(\omega)=\frac{2}{5}\left(\frac{\mathrm{S}^{2}}{\omega^{2}\left\langle\tau_{\mathrm{M}}\right\rangle}+\frac{\left(1-\mathrm{S}_{\mathrm{f}}^{2}\right) \tau_{\mathrm{f}}}{1+\left(\omega \tau_{\mathrm{f}}\right)^{2}}+\frac{\left(\mathrm{S}_{\mathrm{f}}^{2}-\mathrm{S}^{2}\right) \tau_{\mathrm{s}}}{1+\left(\omega \tau_{\mathrm{s}}\right)^{2}}\right)
$$

Table 3.1 Spectral density approaches with adjustable parameters used to fit the relaxation data

\begin{tabular}{clc}
\hline \multicolumn{1}{c}{ Approach } & \multicolumn{1}{c}{ Optimized parameters } & Fixed parameter \\
\hline $\begin{array}{c}\text { Sum of Lorentzian (eq. 3.6) } \\
\text { k: } 1\end{array}$ & $\tau_{1}$ & none \\
2 & $\mathrm{~A}_{1}, \tau_{1}, \tau_{2}$ & none \\
3 & $\mathrm{~A}_{1}, \mathrm{~A}_{2}, \tau_{1}, \tau_{2}, \tau_{3}$ & none \\
& & \\
Lipari-Szabo model-free & $S^{2}, \tau_{\mathrm{M}}, \tau_{\mathrm{e}}$ & none \\
LS (eq. 3.8) & $S^{2}, \tau_{\mathrm{e}}$ & $\tau_{\mathrm{M}}$ \\
& $S^{2}, S_{\mathrm{f}}^{2}, \tau_{\mathrm{M}}, \tau_{\mathrm{s}}, \tau_{\mathrm{f}}$ & none \\
ELS (eq. 3.9) ${ }^{\text {a }}$ & $S^{2}, S_{\mathrm{f}}^{2}, \tau_{\mathrm{s}}, \tau_{\mathrm{f}}$ & $\tau_{\mathrm{M}}$ \\
& $S^{2}, S_{\mathrm{f}}^{2}, \tau_{\mathrm{s}}, \tau_{\mathrm{f}}$ & $\left\langle\tau_{\mathrm{M}}\right\rangle$ \\
\hline
\end{tabular}

${ }^{a}$ Extended Lipari Szabo

${ }^{\mathrm{b}}$ Extended Lipari Szabo with Gaussian distribution 
Note that when $\tau_{M} \gg \tau_{i}$, sum of Lorentzian with $k=2$ is equivalent to LS, $k=3$ to ELS with the following relation: $A_{1}=S^{2}, A_{2}=S_{\mathrm{f}}{ }^{2}-S^{2}, A_{3}=1-S_{\mathrm{f}}{ }^{2}$ and $\tau_{2 / 3}=\tau_{\mathrm{s} / \mathrm{f}}$, respectively.

The fit parameters were calculated via $\chi^{2}$ minimization using in-house MATLAB scripts. The models were assessed using the following criteria: 1) the calculated relaxation parameters are within twice the standard deviation of the experimental values and 2) the calculated error must not be greater than the fitted parameter. ${ }^{122}$ If more than one model meets the criteria, the model with the least $\chi^{2}$ was chosen. $\chi^{2}$ is used to compare the experimental values to calculated values and defined as ${ }^{122-123}$

$$
\chi^{2}=\sum_{\substack{\text { all } \\ \text { frequencies }}}\left[\left(\frac{R_{1 \text { calc }}-R_{1 \exp }}{\sigma_{R 1 \exp }}\right)^{2}+\left(\frac{R_{2 \text { calc }}-R_{2 \exp }}{\sigma_{R 2 \exp }}\right)^{2}+\left(\frac{\mathrm{NOE}_{\text {calc }}-\mathrm{NOE}_{\text {exp }}}{\sigma_{\mathrm{NOE} \exp }}\right)^{2}\right] \text { eq. } 3.20
$$

Best fit parameter were obtained at $\chi^{2}$ minimum per residue. ${ }^{123}$ The uncertainties of the fit parameters were calculated using five hundred Monte Carlo simulations. ${ }^{130,} 139$ Relaxation rates and NOE ratios back calculated from the best fit parameters and experimental uncertainties were used as the mean and standard deviation of the gaussian distribution. Five hundred simulated sets of relaxation rates from the Gaussian distribution were used to calculate simulated model parameters. Reported uncertainties of the best fit parameters are the standard deviations of the simulated model parameters. ${ }^{139}$ The model functions were evaluated using the $\chi^{2}$ goodness of fit test for $\chi^{2}$ distribution with degrees of freedom, $v=n-p$, where $n$ is the number of observed values and $p$ is the number of parameters. For the relaxation data at $\alpha=0.05$ with $v=4$ and 5 , has critical value of 9.49 and 11.07 respectively. Root-mean-square deviation (RMSD) of the calculated relaxation rates from the best fit parameters were also determined. 


\subsection{Results and Discussion}

The disordered nature of minielastin and elastin-like polypeptide monomers has been previously established using NMR techniques. Moreover, in the aggregated state, the structure of elastin-like polypeptides do not drastically change. ${ }^{38}$ The disorder persists in insoluble, mature elastin and that the order parameter for the backbone carbonyl has near zero value. ${ }^{117}$

For minielastin in solution, nuclear spin relaxation of the amide ${ }^{15} \mathrm{~N}$ was used to probe the backbone dynamics of $24 x^{\prime}$. Spin relaxation analysis has been effective in analyzing motions of IDPs. Average values of $1.37 \mathrm{~s}^{-1}$ and $2.54 \mathrm{~s}^{-1}$ for $R_{1}$ and $R_{2}$, respectively, and $\left[{ }^{1} \mathrm{H}\right]-{ }^{15} \mathrm{~N}$ NOE values less than 0.5 at $800 \mathrm{MHz}$ were observed by Zhang and coworkers in a disordered protein, malaria surface protein (MSP2). ${ }^{140}$ This small difference between the $R_{1}$ and $R_{2}$ denotes high flexibility of MSP2. IDPs have a characteristically lower $R_{1}$ and $R_{2}$ and $\left[{ }^{1} \mathrm{H}\right]-{ }^{15} \mathrm{~N}$ NOE values compared to folded proteins. Farrow and coworkers showed $R_{2}$ is lowered as a result of lower contribution from $J(0)$ in the unfolded Drosophila signal transduction protein drk (drkN SH3) compared to folded proteins. ${ }^{137}$

Moreover, study by Bertini and coworkers found that $\alpha$-synuclein, an amyloidogenic IDP, lacks rigidity via model-free approach. ${ }^{141}$ They reported $\mathrm{S}^{2}=0.08 \pm$ 0.02 and rotational correlation time of $7.0 \pm 1.8 \mathrm{~ns}$ for the collective protons of this 140 residue protein using field-cycling relaxometry. Here, $R_{1}, R_{2}$ and heteronuclear NOE were obtained at different magnetic fields to probe the dynamics of minielastin, $24 \mathrm{x}^{\prime}$. 

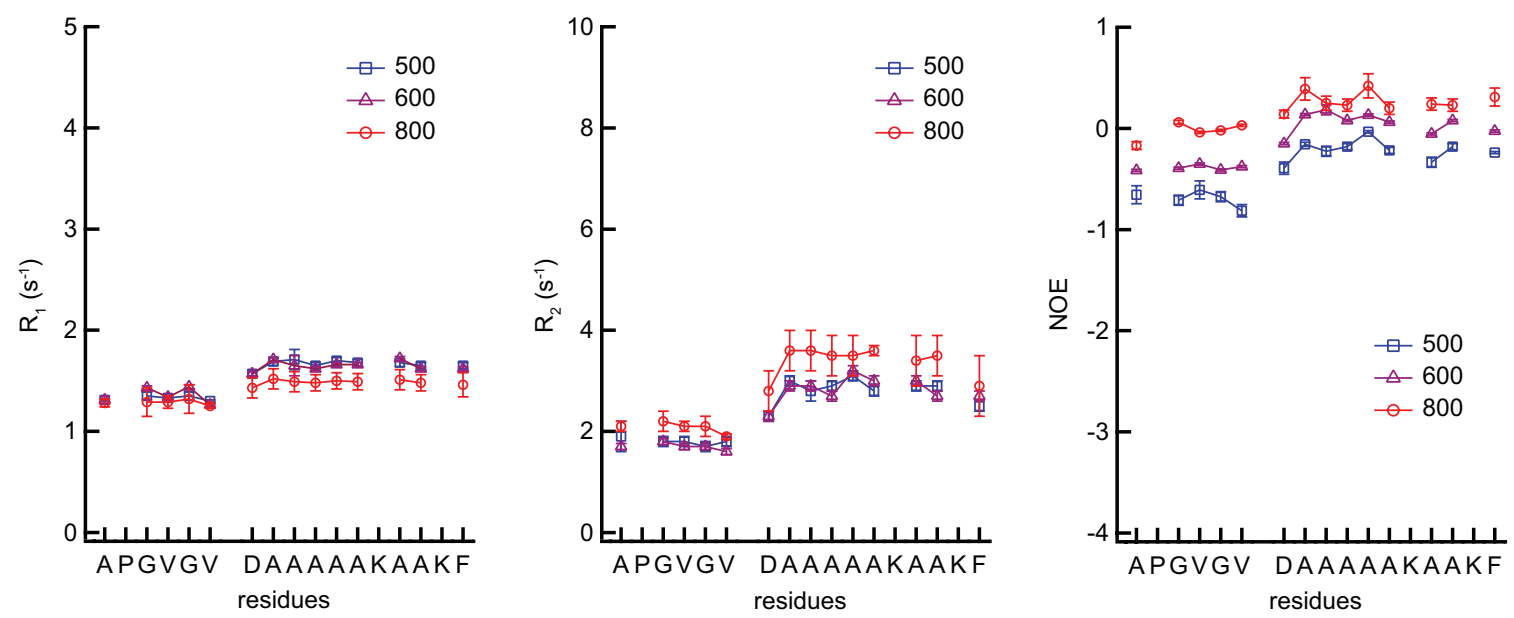

Figure 3.4 NMR spin relaxation data $R_{1}, R_{2}$ and heteronuclear NOE at 500 (blue), 600 $\mathrm{MHz}$ (magenta) and $800 \mathrm{MHz}$ (red) for each residue in $24 \mathrm{x}^{\prime}$.

Shown in Figure 3.4 are the relaxation data obtained at 500, 600 and $800 \mathrm{MHz} . R_{1}$ of hydrophobic module has little to no change between 500 and $800 \mathrm{MHz}$ whereas the cross-link module has significant decrease in $R_{1}$ with increased magnetic field. As shown in eq. 3.3, longitudinal relaxation are contributions of motions in $\omega_{\mathrm{N}}$ and $\omega_{\mathrm{H}} \pm \omega_{\mathrm{N}}$ frequencies. The $R_{1}$ alone is not very informative as it is not sensitive to ps-ns motions. $R_{2}$, on the other hand, is mostly influenced by slow motions and chemical exchange. The difference of $R_{2}$ values between the hydrophobic and cross-link modules support the existence of slower motions for more ordered cross-link module than the flexible hydrophobic module. Relative to $R_{2}$ values of folded proteins (up to $\sim 50 \mathrm{~s}^{-1}$ ), the values obtained were consistent with disordered proteins $\sim 1$ to $6 \mathrm{~s}^{-1} \cdot{ }^{121,128,140,142-143}$ Because of this magnitude, $R_{2}$ values are assumed to have little to no contribution from chemical exchange and thus, are only affected by dipolar coupling and chemical shift anisotropy. Absence of chemical exchange was also confirmed using the CEST experiment by Dr. Sabo. As shown in eq. 3.5, NOEs are sensitive to high frequency motions. Observed values are less than 0.5 consistent with highly flexible motions. Cross-link module shows 
higher NOE than the hydrophobic module. Folded proteins generally have positive NOE values resulted from slower, restricted motions. ${ }^{144}$ Figure 3.4 , in general, shows the striking difference in timescale of motions between the hydrophobic and cross-link modules. Reduced spectral density mapping has been commonly used to describe the dynamics of disordered proteins. Using the relaxation data, spectral density at different frequencies were calculated for each residue. Figure $3.5 \mathrm{a}, \mathrm{b}$ and $\mathrm{c}$ shows the spectral density at $0, \omega_{\mathrm{N}}$, and $0.87 \omega_{\mathrm{H}}$ obtained at 500,600 , and $800 \mathrm{MHz}$. Note that the $J\left(0.87 \omega_{\mathrm{H}}^{500}\right)$ are greater than $J\left(0.87 \omega_{\mathrm{H}}{ }^{600}\right)$ suggests that the plot of the spectral density vs frequency is a summation of Lorentzians at different timescales. ${ }^{137}$ As expected, $J(0)$ and $J\left(\omega_{\mathrm{N}}\right)$ values, for cross-link modules are higher than hydrophobic modules, consistent with more slow motions in the cross-link region. These frequencies are sensitive to nanosecond motions. A slightly higher values of $J\left(0.87 \omega_{\mathrm{H}}\right)$ are observed for hydrophobic residues attributed to more flexibility of the hydrophobic module than the cross-link module. The comparison of average spectral densities of hydrophobic (red) and cross-link (gray) modules in 13 frequencies is shown in Figure 3.6. In summary, there are more low frequency motions in the cross-link modules whereas a slightly greater high frequency motions were observed in the hydrophobic modules. RSDM is a more accurate way to describe the dynamics of disordered proteins because of the broad distribution of motions associated with it. The previous statement is true, there is a compromise between the ease of analysis and the amount of information that can be extracted from it. RSDM does not provide a physical picture of protein dynamics, thus, the importance of spectral density modelling. 
Relaxation is a process caused by the combination of small motions rather than one large motion. ${ }^{118}$ These contributions of motions are embedded in the relaxation measurements as shown in eqs. $3.3-3.5$. Gill and coworkers used both spectral density mapping and the model-free approach to analyze the dynamics of transcription factor

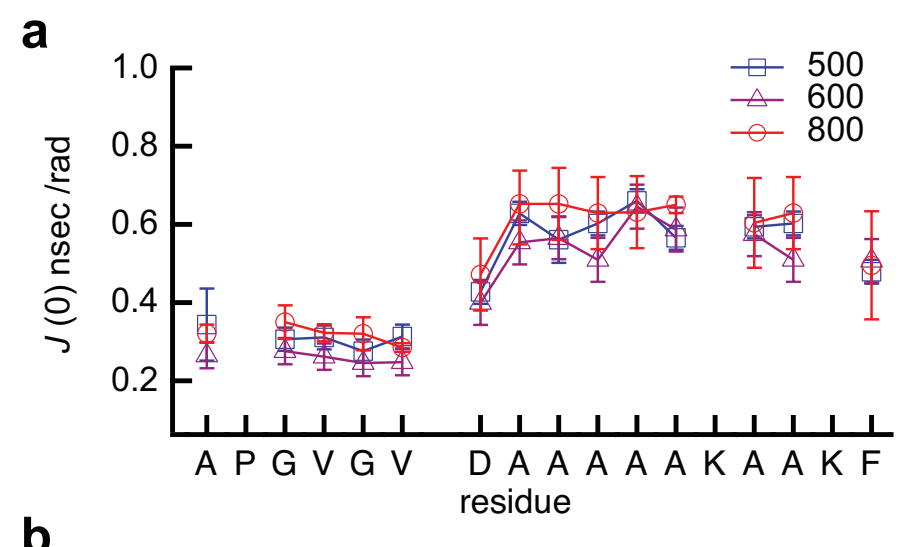

b
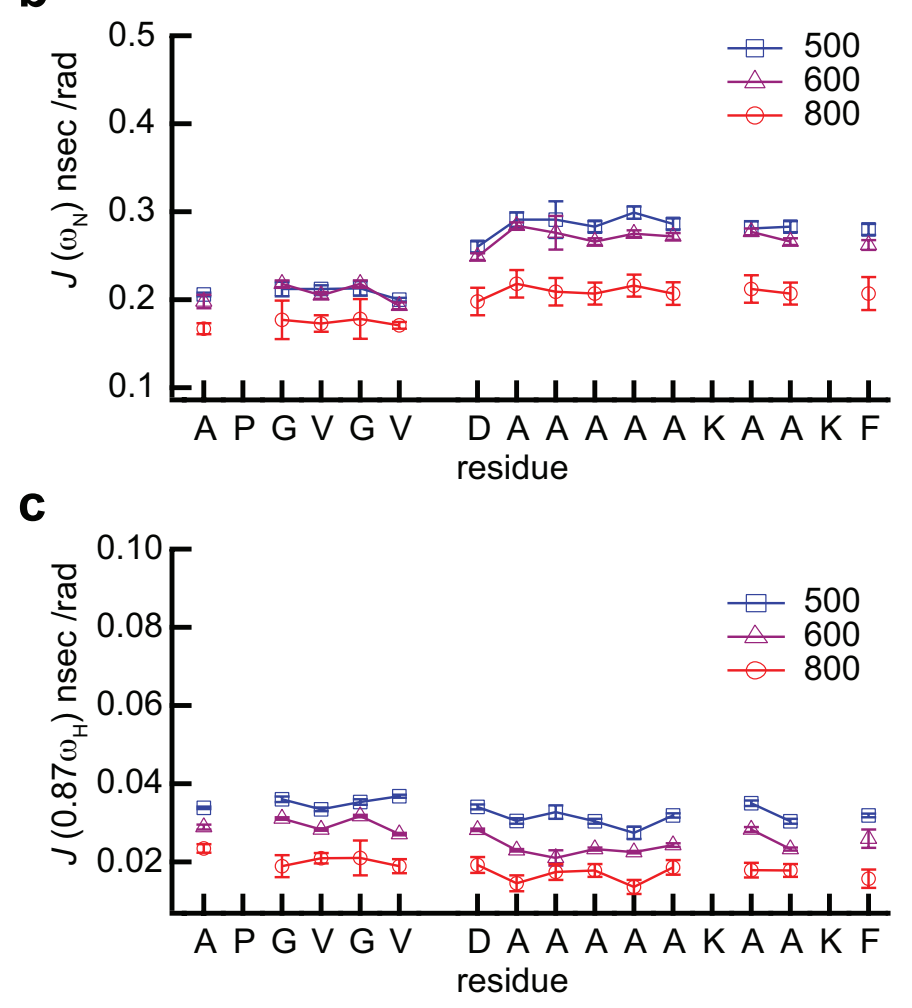

Figure 3.5 Spectral density calculated from the NMR spin relaxation data at a) $J(0)$ averaged at three magnetic fields, b) $J(\omega \mathrm{N})$ and $J(0.87 \omega \mathrm{H})$ obtained from 500 (open square), 600 (open triangle) and $800 \mathrm{MHz}$ (open circle) magnetic fields. 


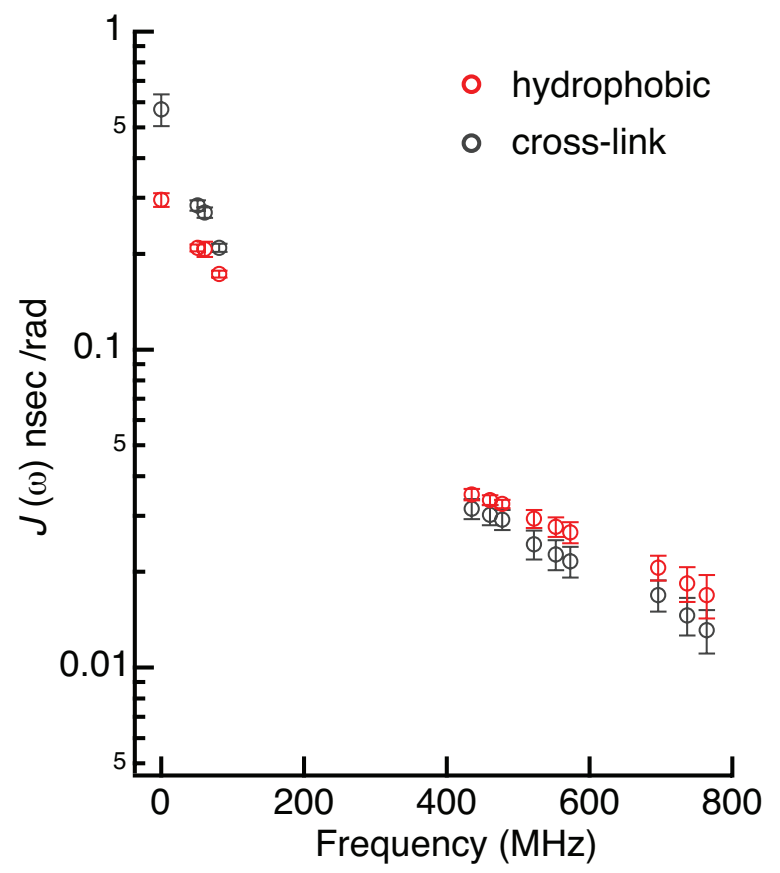

Figure 3.6 The average spectral density functions for hydrophobic (red) and cross-link (gray) were sampled at $0, \omega \mathrm{N}, 0.87 \omega \mathrm{H}, 0.921 \omega \mathrm{H}$, and $0.955 \omega \mathrm{H}$ at 500,600 and 800 MHz. ${ }^{137}$

GCN4 protein. ${ }^{121}$ The relaxation data of the disordered region of GCN4 protein was fitted using extended Lipari-Szabo formalism and yielded spectral density similar to that calculated using spectral density mapping. Similarly, Abyzov et al. used spin relaxation at multiple magnetic field and different temperatures to study the dynamic modes of the disordered C-terminal domain of Sendai Virus nucleoprotein. Using the six-parameter model of the spectral density function, they were able to distinguish three dynamic modes that were attributed to the fast librational motions ( $\sim 50 \mathrm{ps})$, intermediate local backbone $(\sim 1 \mathrm{~ns})$ and segmental motions $(\leq 20 \mathrm{~ns}) .{ }^{145}$ More recently, they have shown the important role of solvent in segmental motions of IDP via MD simulations. ${ }^{128}$ 
Table 3.2 Fit parameters obtained from sum of Lorentzian with $\mathrm{k}=2$ and 3 at $\chi^{2} \mathrm{~min}$.

\begin{tabular}{|c|c|c|c|c|c|c|c|c|}
\hline residue & $\mathbf{k}$ & $\mathbf{A}_{1}$ & $\tau_{1}(\mathbf{n s})$ & $\mathbf{A}_{2}$ & $\tau_{2}(\mathrm{~ns})$ & $\mathbf{A}_{3}$ & $\tau_{3}(\mathbf{p s})$ & $\chi^{2}$ \\
\hline \multirow[t]{2}{*}{$\mathrm{A}(1,24)$} & 2 & & & 0.352 & 1.80 & 0.648 & 89 & 12.6 \\
\hline & 3 & 0.007 & 12.7 & 0.393 & 1.44 & 0.600 & 84 & 4.25 \\
\hline \multirow[t]{2}{*}{$\mathrm{G}(3,24)$} & 2 & & & 0.585 & 1.01 & 0.415 & 68 & 17 \\
\hline & 3 & 0.003 & 28.7 & 0.603 & 0.97 & 0.394 & 63 & 7.7 \\
\hline \multirow[t]{2}{*}{$\mathrm{G}(5,24)$} & 2 & & & 0.539 & 1.09 & 0.461 & 81 & 8.2 \\
\hline & 3 & 0.003 & 35.3 & 0.543 & 1.26 & 0.543 & 74 & 6.1 \\
\hline \multirow[t]{2}{*}{$\mathrm{D}\left(1, \mathrm{x}^{\prime}\right)$} & 2 & & & 0.48 & 1.69 & 0.52 & 94 & 21.7 \\
\hline & 3 & 0.029 & 10.2 & 0.535 & 1.31 & 0.436 & 83 & 3.2 \\
\hline
\end{tabular}

To extract the amplitudes and timescales of motions that affects the relaxation, several approaches were used to fit the experimental values. For the sum of Lorentzian models, at $\mathrm{k}=3$, all residues fitted the criteria that were previously established whereas at $\mathrm{k}=2$, only four residues resulted to acceptable fits as shown in Table 3.2. Note that the fit parameters for the internal motions are similar but the $\chi^{2}$ minimum obtained from $\mathrm{k}=$ 3 is lower than at $\mathrm{k}=2$. The slowest correlation time at $\mathrm{k}=2$ is physically impossible to be the overall orientation time but can be attributed to local slow motions. NOEs at $\mathrm{k}=2$ are smaller than the experimental values contributing to higher calculated $\chi^{2}$.

The fit parameters with calculated relaxation values within two standard deviations of the experimental values for A $\left(1,24^{\prime}\right)$ with $\chi^{2}$ values depicted by the 
a

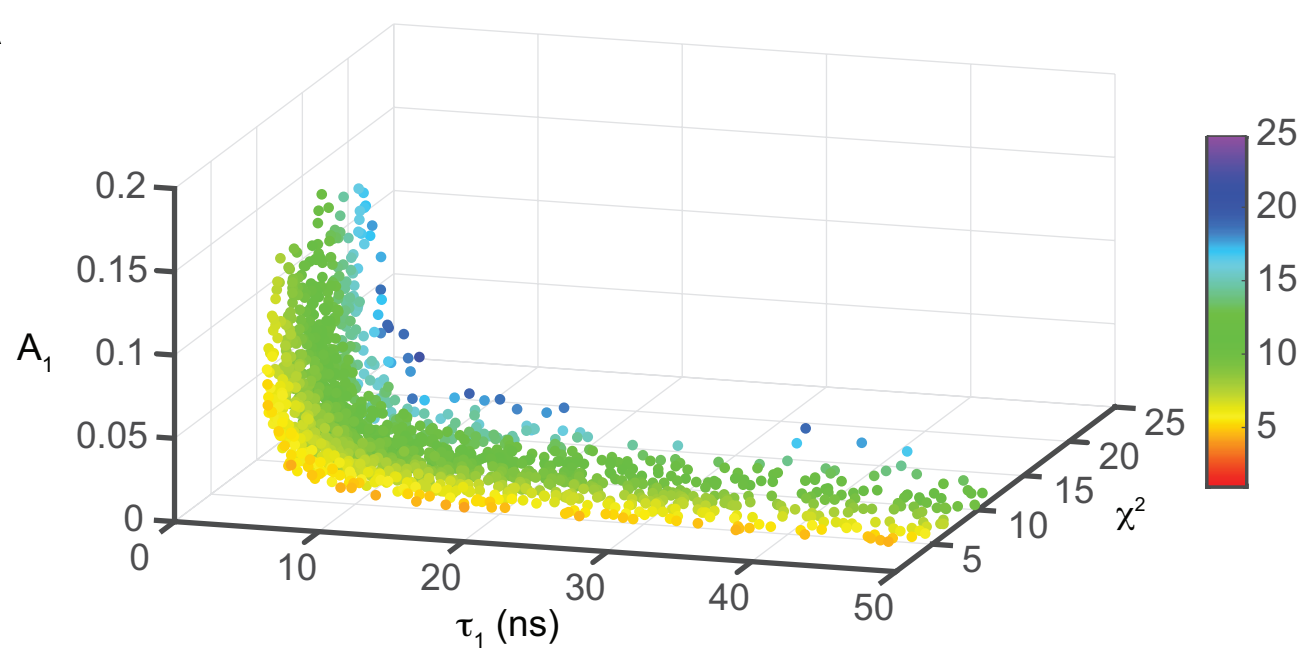

b

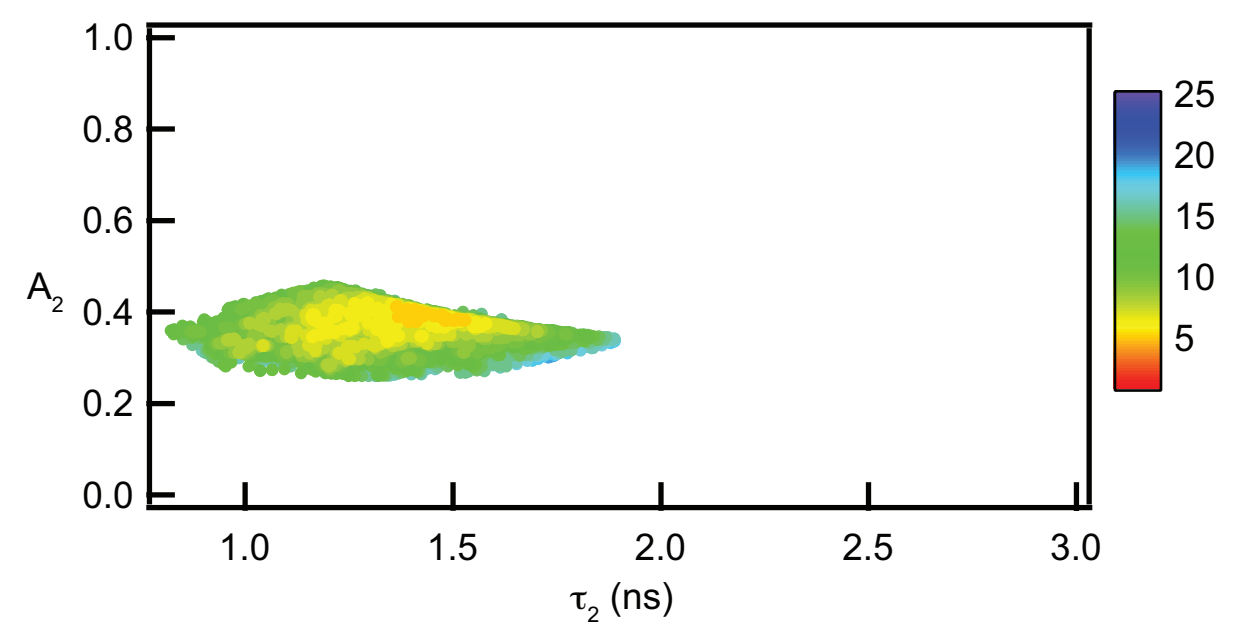

C

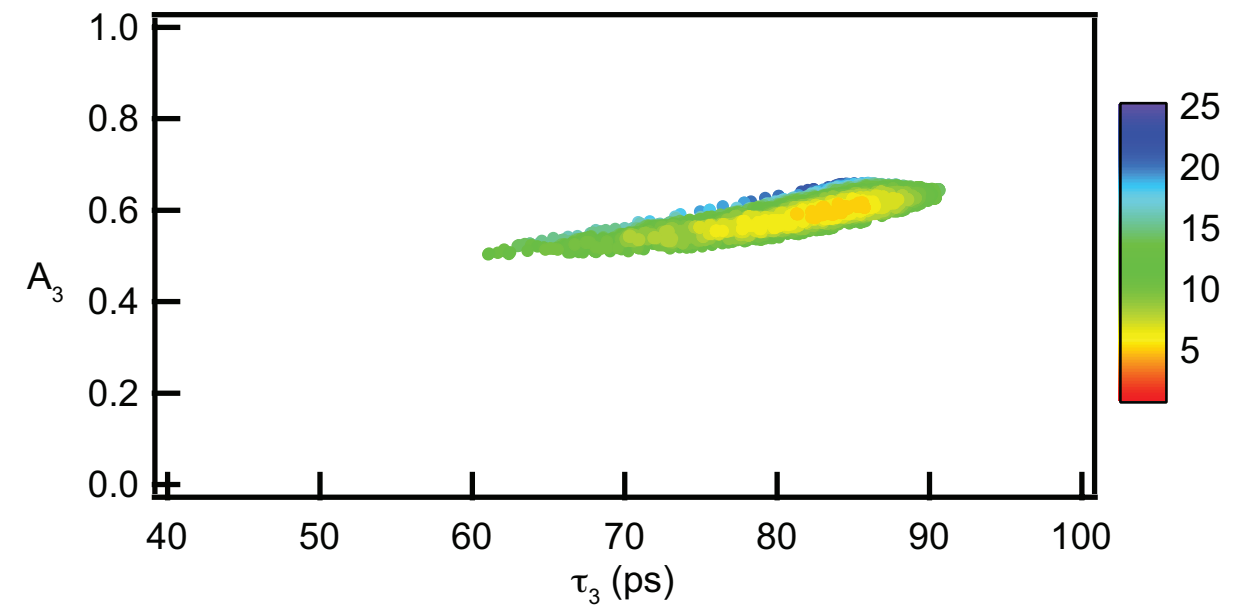

Figure 3.7 Fit parameters of A $\left(1,24^{\prime}\right)$ with calculated relaxation values within two standard deviation of the experimental values obtained from sum of Lorentzian with $\mathrm{k}=$ 3. $\chi^{2}$ values indicated by the colormap. 
colormap are shown in Figure 3.7. A non-linear relationship between the slowest timescale $\tau_{1}$ and coefficient $A_{1}$ was observed, with $A_{1}$ ranges from 0 to 0.25 for $3 \leq \tau_{1} \leq$ $50 \mathrm{~ns}$. This wide range of values is consistent with a distribution of overall rotational correlation time associated with disordered proteins. $\tau_{2}$ and $\tau_{3}$ have a linear relationship with their respective coefficients as shown in Figure $38 \mathrm{~b}$ and c. These fit parameters show how much each timescale affect the relaxation rates. Small contributions from the slowest correlation time ( $>3 \mathrm{~ns}$ ) were observed as well as major contributions from fastest motions $(<100 \mathrm{ps})$. Given the range of timescales used, at $\left(\omega \tau_{1}\right)^{2}>>1$ and $\left(\omega \tau_{2}\right)^{2}$ $<<1$, eq. 3.6 simplifies to

$$
J(\omega)=\frac{2}{5}\left(\frac{\mathrm{A}_{1}}{\omega^{2} \tau_{1}}+\frac{\mathrm{A}_{2} \tau_{2}}{1+\omega^{2} \tau_{2}{ }^{2}}+\mathrm{A}_{3} \tau_{3}\right)
$$

The correlation function decays fast at the slowest timescale and the third term no longer depend on frequency. Figure 3.8 summarizes the fit parameters obtained per residue. In red are the values from Lorentzian $\mathrm{k}=3$ model and in blue are from fitting with GELS. Understanding of the physical representation of minielastin dynamics from the spectral density was further analyzed using the model-free approach. LS has been commonly used to describe the dynamics of folded, denatured and disordered proteins. ${ }^{121-123,}{ }^{146}$ The square of the generalized order parameter $\left(S^{2}\right)$ describes the spatial restriction of the $\mathrm{NH}$ bond vector with timescales obtained with minimal assumption on the nature of these motions. LS assumes the separation of the timescale of the overall tumbling and internal motions whereas the Lorentzian model provided the contributions or weighing of each timescale.

Fit parameters acquired using ELS approach are in good agreement with the sum of Lorentzian with $\mathrm{k}=3$ as discussed previously. In eq. 3.19 , when $\tau_{\mathrm{M}}>>\tau_{\mathrm{f}}, \tau_{\mathrm{f}}^{\prime}$ reduces to 
$\tau_{\mathrm{f}}$ and the effect of $\tau_{\mathrm{M}}$ is more evident in $\tau_{\mathrm{S}}$ term as $\tau_{\mathrm{M}}$ is not much bigger than $\tau_{\mathrm{S}}$. A wide range of overall correlation time and generalized order parameters were observed. The zero-frequency term of the spectral density is highly dependent on the $\mathrm{S}^{2}$, given that the overall correlation time is much slower relative to the fast internal motions. The accurate calculation of $S^{2}$ was attempted by setting the overall correlation time constant with values at least an order of magnitude slower than the internal correlation time. Correlation times were varied based on the average $\tau_{1}$ observed from $\mathrm{k}=3$. Using $\tau_{\mathrm{M}}=4$ ns, value consistent with segmental motions, the fit parameters drastically change but as expected, not all residues resulted an acceptable fit. For the hydrophobic modules, the change is evident in $\mathrm{S}^{2}$ and $\mathrm{S}_{\mathrm{s}}^{2}$ whereas $\mathrm{S}_{\mathrm{f}}^{2}$ is unchanged. In addition, $\tau_{\mathrm{s}}$ values increased and $\tau_{\mathrm{f}}$ is about the same. These suggests that the changes in the slow internal motions do not affect the fast internal motions. The $\tau_{\mathrm{M}}=4 \mathrm{~ns}$ for cross-link module yielded $\mathrm{S}^{2}<0.2$, increased $\mathrm{S}_{\mathrm{s}}{ }^{2}$ and $\mathrm{S}_{\mathrm{f}}{ }^{2}$ and decreased $\tau_{\mathrm{s}}$ and $\tau_{\mathrm{f}}$ values. Note that the $\chi^{2}$ also increased at these fit parameters, suggesting poorer fitting.

An estimate of $\tau_{\mathrm{M}}$ was calculated from the hydrodynamic radius obtained via PFG NMR. Hydrodynamic radius, a good estimate of the radius of the molecule via Stokes equation, is related to the rotational correlation time as shown in eq. 3.17. Figure 3.9 shows how the order parameters vary with overall correlation time. At value estimated from hydrodynamic radius, $\tau_{\mathrm{M}} \sim 40 \mathrm{~ns}, \mathrm{~S}^{2}<0.005$ for the hydrophobic modules and less than 0.02 for the cross-link module. It does not provide additional information about the flexibility of the molecule rather affects the order parameter of the slow internal motions, $\mathrm{S}_{\mathrm{s}}{ }^{2}$. The order parameter for the slow and fast internal motions were extracted at constant $\tau_{\mathrm{M}}$, Figure 3.9. At these $\mathrm{S}^{2}$ for the hydrophobic and cross-link module, $\mathrm{S}_{\mathrm{s}}^{2}$, are rather 
small compared to $\mathrm{S}_{\mathrm{f}}^{2}$ therefore, the order parameter for motions slower than $\sim 0.80 \mathrm{~ns}$ are very small and that these motions are not spatially restricted. When $\tau_{M}$ is set to $19 \mathrm{~ns}$, average calculated overall correlation time from the ELS fitting for all residues, fitting parameters are unchanged.
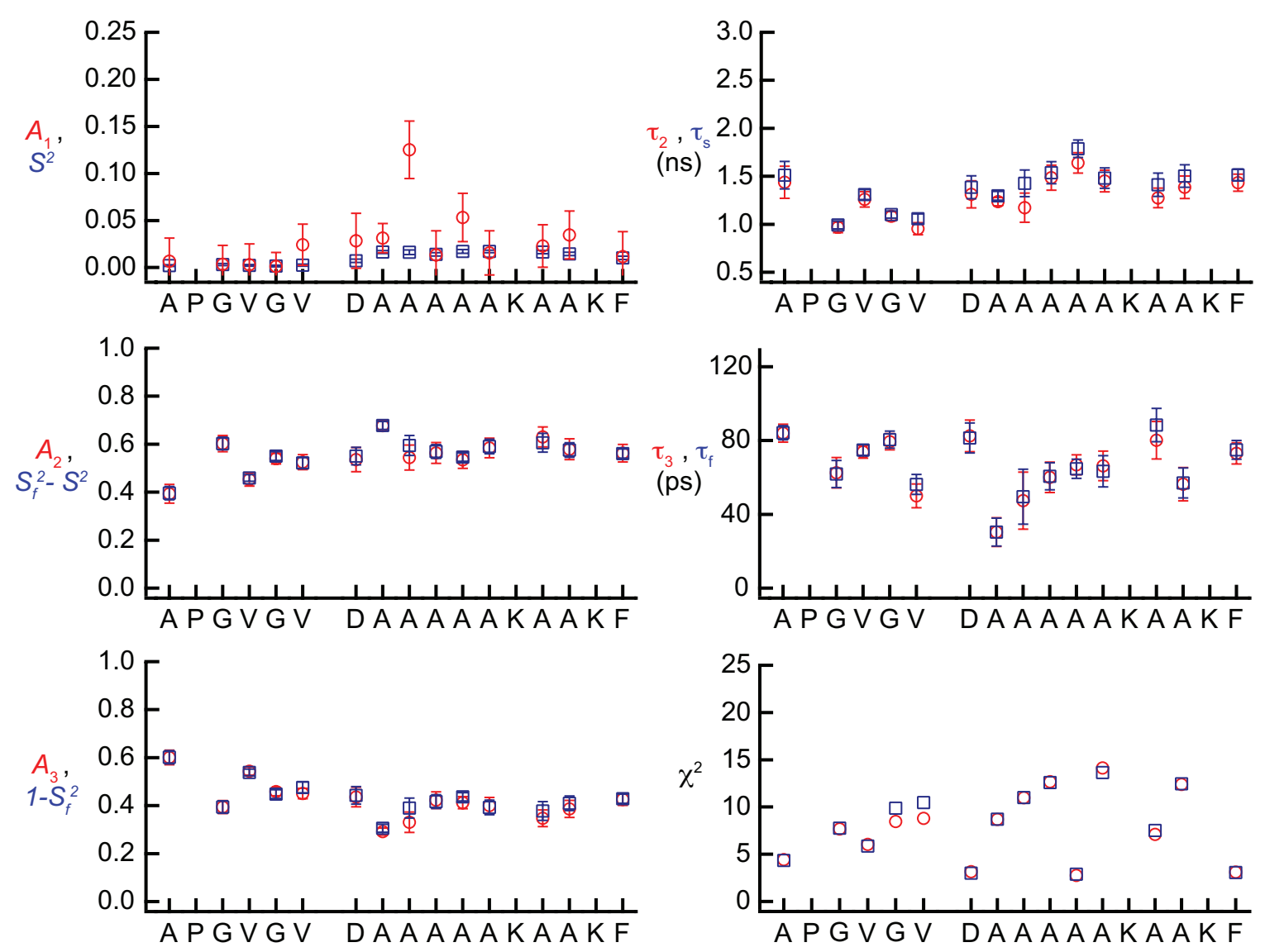

Figure 3.8 Fit parameters obtained for sum of Lorentzian model with $\mathrm{k}=3$ (red circles) and extended Lipari-Szabo with Gaussian distribution (blue squares). 

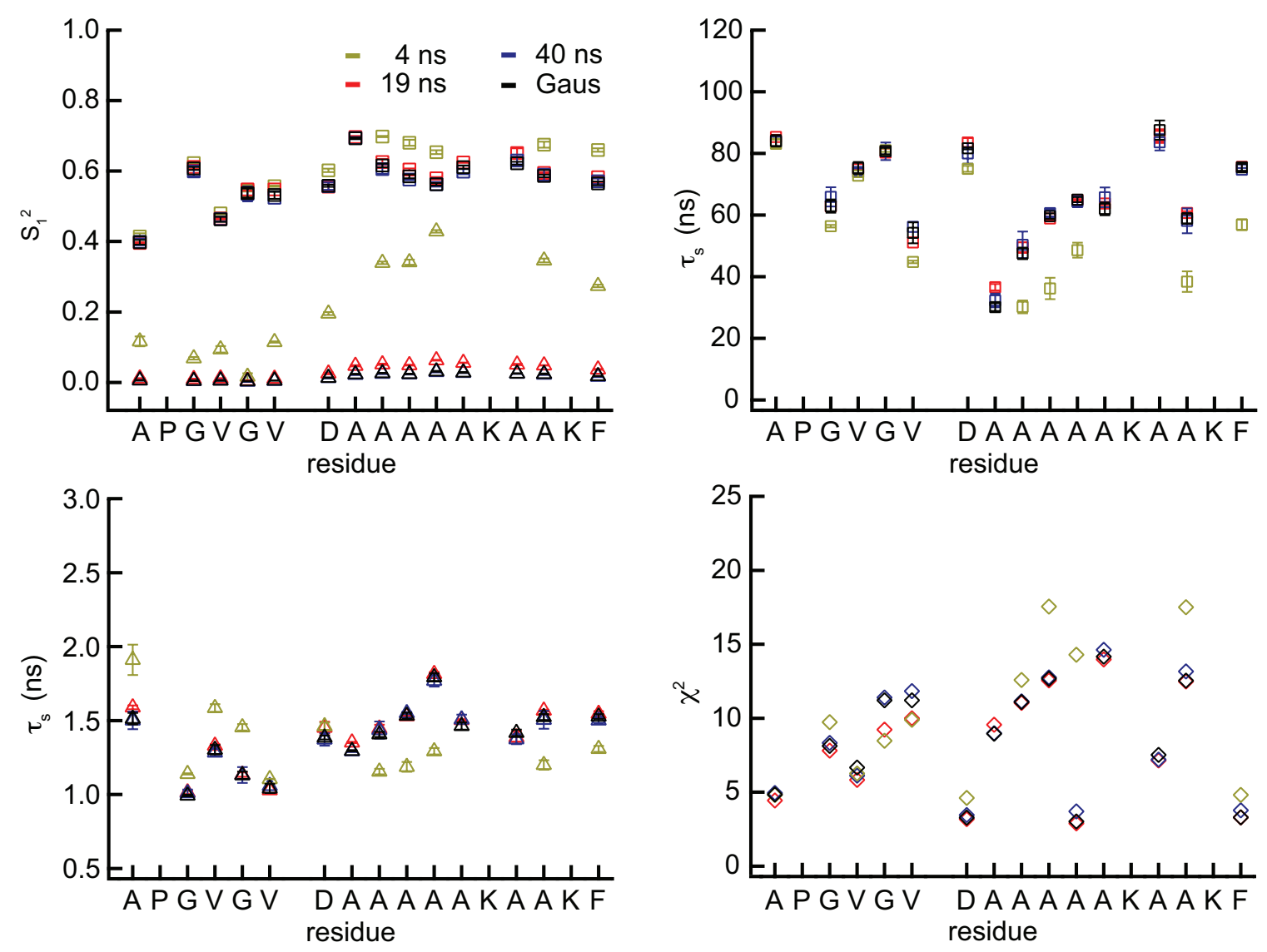

Figure 3.9 ELS parameters at $\tau_{\mathrm{M}}$ equal to $4 \mathrm{~ns}$ (yellow), $19 \mathrm{~ns}$ (red), $40 \mathrm{~ns}$ (blue) and $\tau \mathrm{M}$ with Gaussian distribution (black). In top left are the $\mathrm{S}_{\mathrm{s}}{ }^{2}$ and $\mathrm{S}_{\mathrm{f}}{ }^{2}$ values given that $\mathrm{S}^{2}=\mathrm{S}_{\mathrm{s}}{ }^{2}$ $\mathrm{S}_{\mathrm{f}}{ }^{2}$.

Lastly, a Gaussian distribution of the overall correlation times is applied. As previously established, minielastin is intrinsically disordered and thus, samples a range of hydrodynamic radii. The Gaussian distribution was applied to the hydrodynamic radii with a full width at half height (FWHH) of $10 \AA$ that was found for other intrinsically disordered proteins of similar molecular weight. ${ }^{147-148}$ The distribution was centered at a $\tau_{\mathrm{M}}$ of $\sim 40 \mathrm{~ns}$ calculated from the Stokes-Einstein relation using the hydrodynamic radius for $24 \mathrm{x}^{\prime}, 34 \AA .^{30}$ This distribution has $\tau_{\mathrm{M}}$ values greater than 15 ns. Fits of this ELS approach with a Gaussian distribution resulted in $0<\mathrm{S}^{2}<0.04$, and values $\mathrm{S}_{\mathrm{f}}{ }^{2}, \tau_{\mathrm{f}}$ and $\tau_{\mathrm{s}}$ 
essentially unchanged compared to fit parameters when $\tau_{\mathrm{M}}$ is constrained to 19 and $40 \mathrm{~ns}$. With $\tau_{\mathrm{M}}$ values at least one order of magnitude slower than $\tau_{\mathrm{s}}, \mathrm{S}_{\mathrm{s}}{ }^{2}, \mathrm{~S}_{\mathrm{f}}^{2}$ and corresponding internal correlation times are constant.

The order parameters with $\tau_{\mathrm{M}} \gg \tau_{\mathrm{S}}$ are consistent with the values obtained from the backbone carbonyl of mature elastin with $\mathrm{S}<0.1 .{ }^{117}$ This shows that the amplitudes of motions are conserved from minielastin to mature elastin. With the order parameters conserved, it is expected that the timescale of motions of elastin dynamics are elevated brought about by intensive cross-linking. It is important to note that the degree of disorder presented here is necessary in the recoil mechanism of elastin and that coacervation of monomers do not increase the backbone ordering. Reichheld and coworkers demonstrated using ${ }^{15} \mathrm{~N}-{ }^{1} \mathrm{H}$ HSQC of a similar minielastin construct that chemical shifts are retained with line broadening due to coalescence of monomers. ${ }^{38}$

Various models were explored to describe the backbone dynamics of minielastin molecules in solution. The sum of Lorentzian models and Lipari-Szabo model-free approaches were used in parallel with reduced spectral density mapping. Figure 3.10 shows that fitting parameters obtained from the Lorentzian with $\mathrm{k}=3$ can describe the spectral density map from hydrophobic and cross-link residues without the assumption associated with LS. Three terms were found necessary to fit the spectral density because of the slightly positive NOE values. $\chi^{2}$ min and corresponding RMSD values (see Appendix II-B) were calculated to show the accuracy of the fitting parameters. Most residues resulted to values within the critical limit for both $\mathrm{k}=3$ and GELS approaches. For residue with best fit parameters that are statistically rejected such as $\mathrm{A}\left(6, \mathrm{x}^{\prime}\right)$, possibility that the model used was incorrect can be excluded given the good agreement 
between the RSDM and the calculated spectral density from the best fit parameters, Figure 3.10. It is possible that the errors were underestimated that resulted to high $\chi^{2}$ value.
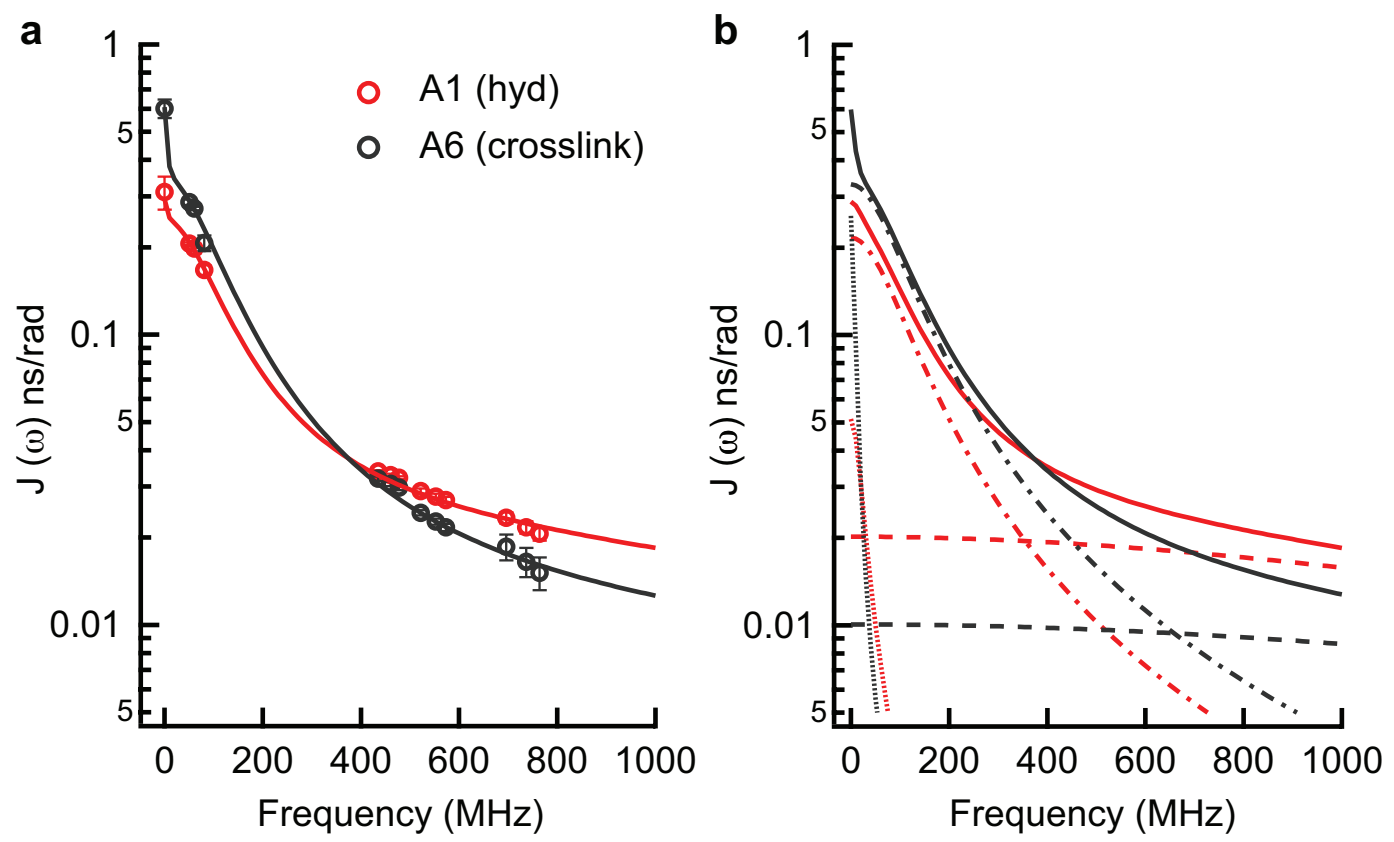

Figure 3.10 (a) Spectral density function of $\mathrm{A}(1,24)$ and $\mathrm{A}\left(6, \mathrm{x}^{\prime}\right)$ calculated from experimental relaxation data using method 3 in red and black circles, respectively. Spectral density function with fit parameters calculated using eq. 3.6 are shown in solid lines. (b) The contributions of each timescale; (…) slowest, (- - - -) intermediate, and (- - -) fast timescale, to the spectral density of A $(1,24)$ in red and A $\left(6, x^{\prime}\right)$ black solid lines.

Three different approaches have shown that the minielastin $24 x^{\prime}$ in solution is highly flexible with $S^{2}<0.04$ when $\tau_{\mathrm{M}} \gg \tau_{\mathrm{S}}>>\tau_{\mathrm{f}}$. Small contribution from the slowest correlation time is important to obtain the best fit dynamical parameters. The hypothesis is that the high flexibility of minielastin monomer that is conserved in the coacervate and the cross-linked mature elastin is important in the protein function of elastin. It is important to note that some intrinsically disordered proteins are known to form secondary structures upon binding or oligomerization whereas minielastin remains disordered. 


\section{CHAPTER 4. MINIELASTIN FORMS AN INSOLUBLE CROSS-LINKED PRODUCT WITH GENIPIN}

\subsection{Introduction}

Elastin, an extracellular matrix protein, is a vital component of elastic fibers and a natural polymer responsible for elastic properties of tissues. Mature elastin is composed of a network of cross-linked tropoelastin molecules that provides resilience from proteolytic degradation and, thus, long lifetime. The organization and amount of elastin in the elastic fiber varies depending on the directionality of deformation and the extent of elasticity needed for tissue function. ${ }^{149}$

Tropoelastin monomers are transported by a $67 \mathrm{kDa}$ elastin binding proteins (EBP) to the outside of the cell. EBP prevents proteolysis and premature coacervation of tropoelastin extracellularly. ${ }^{150}$ EBP dissociates with tropoelastin and is recycled in the process. Tropoelastin undergoes coacervation followed by cross-linking facilitated by lysyl oxidase (LOX) that converts primary amines of lysyl sidechains into an aldehyde groups. ${ }^{78}$ Elastin cross-links are found to be bi-, tri- and tetra functional, connecting two, three and four lysine sidechains, respectively. Bifunctional cross-links form allysine aldol and lysinonorleucine whereas trifunctional cross-links form merodesmosine. Finally, the tetrafunctional cross-link desmosine is found to be prevalent in mature elastin. ${ }^{151-152}$ Protein aggregates are deposited to microfibril scaffolds where further cross-linking and 
maturation occurs. In mature elastin, $>80 \%$ of lysines are cross-linked. ${ }^{153}$ Schmelzer and coworkers have recently shown that elastin undergoes heterogeneous cross-linking contrary to the specific cross-links that were proposed by Baldock and coworkers. ${ }^{114-115}$, 154-155

\subsubsection{Cross-linking}

An important characteristic of tropoelastin and minielastins is the ability to form insoluble cross-linked products. Cross-linking is the formation of interchain covalent bonds that stabilize the polymeric network structure. ${ }^{15}$ Several cross-linking methods have been applied to tropoelastin and elastin-like proteins (ELP) to synthesize elastic biomaterials. For example, Urry and coworkers used $\gamma$-irradiation by cobalt $60^{20}$ to crosslink ELPs, whereas Zhang and coworkers flanked ELPs with cysteine residues and induced disulfide bonds cross-linking by UV light. ${ }^{156}$ Constructs similar to minielastins were cross-linked by Vieth and coworkers using genipin and pyrroloquinoline quinone (PQQ). PQQ catalyzes the oxidative deamination of primary amines, a cross-linking mechanism similar to LOX, Figure 4.1. The structure of PQQ resembles the active site of LOX with lysyl tyrosylquinone (LTQ) cofactor, Figure 4.2. The advantage of using PQQ as a cross-linker is the production of the tetrafunctional cross-link, desmosine. Moreover,

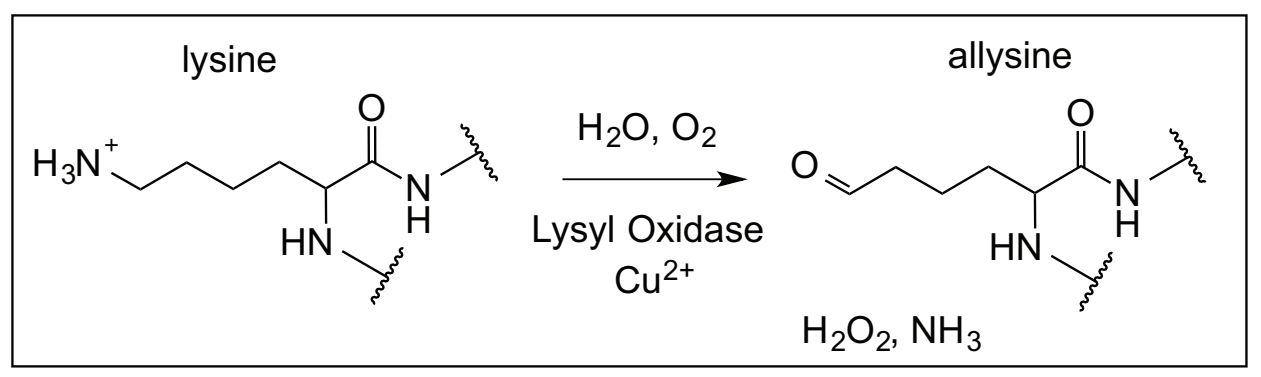

Figure 4.1 Lysyl oxidase catalyzes the oxidative deamination of lysine side chain and yields allysine, hydrogen peroxide and ammonia. ${ }^{155}$ 


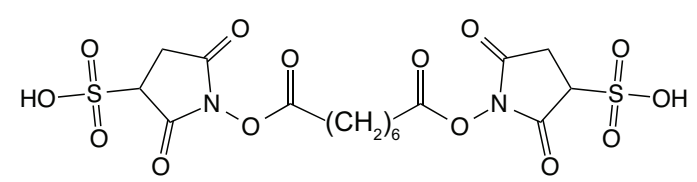

Bis(sulfosuccinimidyl)suberate

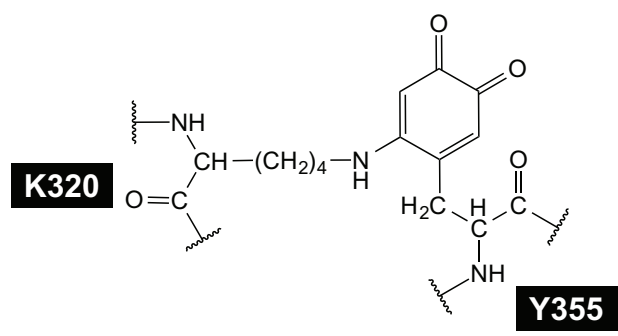

lysyl tyrosylquinone

(LTQ, lysyl oxidase active site)

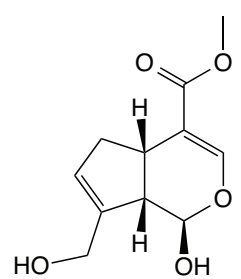

genipin

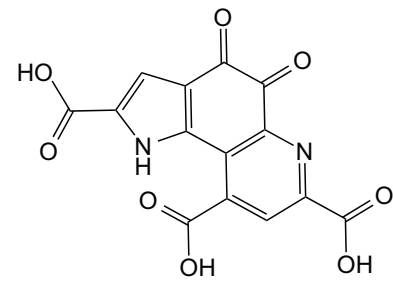

pyrroloquinoline quinone (PQQ)

Figure 4.2 Several cross-linkers used for tropoelastin and ELP cross-linking. Bis(sulfosuccinimidyl)suberate ${ }^{157}$ is a bifunctional cross-linker whereas genipin, ${ }^{15}$ lysyl oxidase $^{158}$ and $\mathrm{PQQ}^{15,159}$ yields bi-, tri- and tetrafunctional cross-links.

Vieth and coworkers showed that the biopolymers made using genipin produced a crosslinked material with greater tensile strength than materials cross-linked with PQQ. ${ }^{15}$

\subsubsection{Genipin}

Genipin has been widely used as a natural cross-linker for primary amines as a less cytotoxic alternative to synthetic cross-linkers such as glutaraldehyde. ${ }^{160}$ Genipin is derived from the hydrolysis of geniposide isolated from the fruits of Genipa americana and Gardenia jasminoides Ellis. ${ }^{161}$ It has gained popularity as a food coloring agent since the reaction of genipin with primary amines produces a blue pigment. Moreover, cross-linking with chitosan, collagen and gelatin demonstrated the potential of genipin as 
a<smiles>CC(=O)C1=CN(C)C=C2C(CO)=CCC12</smiles>

d

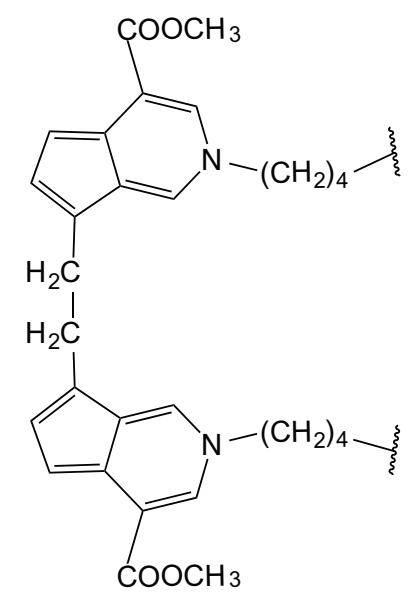

b<smiles>CC(=O)c1cn(C)cc2c(C)ccc1-2</smiles>

C

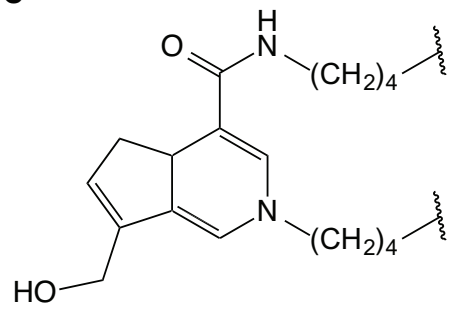

e

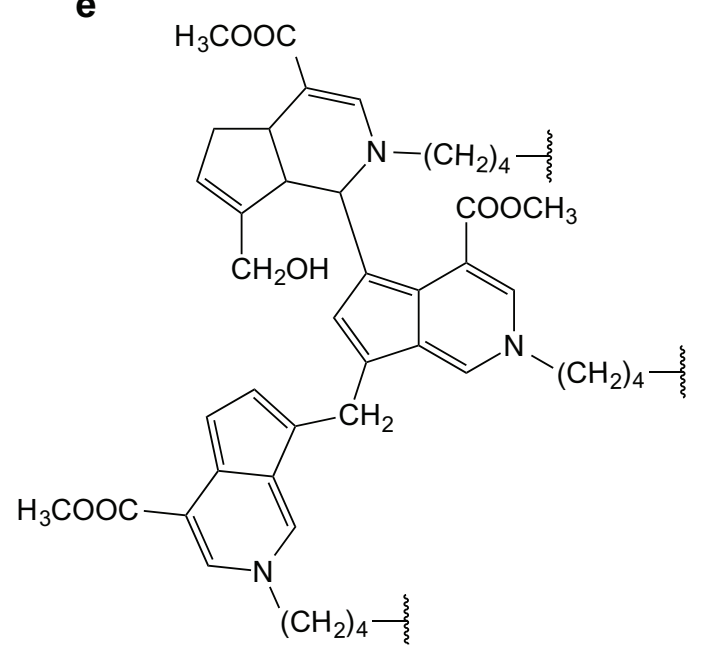

Figure 4.3 Reaction of genipin with primary amines yields a) yellow and b) brownish-red intermediates. Proposed cross-linked products based on the mechanisms of c) Butler and coworkers ${ }^{162}$ and d-e) Touyama and coworkers. ${ }^{163-164}$

a tissue fixation agent. ${ }^{165-168}$ Several mechanisms ${ }^{162,169-170}$ have been proposed and crosslinked intermediates and products $^{163-164}$ have been elucidated but the structure identification of the high molecular weight polymer that consists of $\sim 40-44$ monomer units ${ }^{163}$ have been elusive. Blue pigments were found to be soluble in water, ethanol and methanol and were formed in the presence of oxygen.

In this study, genipin, a natural cross-linking reagent, was used to cross-link the minielastin construct, $24 \mathrm{x}^{\prime}$. Genipin is nontoxic, biocompatible and is known to produce more cross-links than PQQ. Minielastin construct, 24x', like tropoelastin, forms an insoluble, cross-linked product when genipin is the cross-linking reagent. Since genipin 
undergoes polymerization, Figure $4.3 \mathrm{~d}$ and e, the length between cross-link modules varies, unlike cross-linking with LOX or PQQ where cross-linked modules are separated by the length of two lysyl sidechain.

\subsection{Materials and Methods}

Lyophilized minielastin $(2 \mathrm{mg}), 24 \mathrm{x}^{\prime}$, was dissolved in $0.15 \mathrm{M}$ sodium borate buffer $\mathrm{pH} 8.0$ at $4{ }^{\circ} \mathrm{C}$ overnight. In a $3 \mathrm{~mm}$ tube, $16 \mu \mathrm{L}$ of $5 \mathrm{M} \mathrm{NaCl}$ solution was added to $74 \mu \mathrm{L}$ of a minielastin solution to induce coacervation. $10 \mu \mathrm{L}$ of $100 \mathrm{mM}$ genipin solution, prepared by dissolving $2.27 \mathrm{mg}$ genipin in $100 \mu \mathrm{L}$ ethanol, was added to the solution. The sample was inserted in a prewarmed centrifuge for 7 minutes at 6,000 rpm $\left(2060 \times g, 37^{\circ} \mathrm{C}\right)$ and left to incubate at $37^{\circ} \mathrm{C}$ overnight. This cross-linking protocol was based on the optimized method for ELPs published by Muiznieks. ${ }^{171}$ To show that genipin does not form insoluble products from reaction with primary amines and polymerization alone, $12 \mathrm{mg}$ BOC-glycine, glycine and lysine were dissolved in $20 \mu \mathrm{L} 5 \mathrm{M} \mathrm{NaCl}, 20 \mu \mathrm{L}$ genipin and $160 \mu \mathrm{L}$ buffer (pH 6 phosphate buffer or $\mathrm{pH} 8$ borate buffer). Two buffer solutions were utilized to investigate the effect of $\mathrm{pH}$ on the cross-linking of amino acids. Note that Tris-based buffers cannot be used since they contain a primary amino group. 


\subsection{Results and Discussion}

Cross-linking is an important feature of the elastic fiber facilitated in vivo by the enzyme, lysyl oxidase. Extensive cross-linking of tropoelastin provides elastin the structural integrity and resistance to proteolytic cleavage. Several designed ELPs have been used to synthesize insoluble elastic material with various cross-linking methods. ELPs containing only the hydrophobic modules were cross-linked via radical polymerization or by addition of cross-linking residues i.e., amine- or sulfide-containing residues. Tropoelastin and minielastins, on the other hand, contain cross-linking modules characterized by the $\mathrm{A}_{4 / 5} \mathrm{KA}_{2 / 3} \mathrm{~K}$ moiety. Bellingham and coworkers showed that minielastins with at least three hydrophobic modules and two cross-link modules can produce an elastic insoluble material. ${ }^{14}$ Genipin, a natural cross-linker that reacts with primary amines, was used to synthesize insoluble cross-linked $24 x^{\prime}$, a construct with three crosslink modules flanked by four hydrophobic modules.

To confirm that genipin specifically reacts to primary amines, preliminary tests with BOC-glycine, glycine and lysine amino acids reacted with genipin were performed. In Figure 4.4, initial reaction of primary amines, glycine and lysine, with genipin were observed to yield a soluble yellow product, that after an hour, forms a brownish - red solution. Touyama and coworkers characterized the yellow and brownish-red intermediates, Figure 4.4 (10 min and $1 \mathrm{hr}$ ), as the dihydropyridine compounds shown in Figure 4.3a and b. ${ }^{163-164}$ After 22 hours, the indigo blue oxidation products of genipin described by Touyama are observed. Note that even with genipin polymerization, the blue products were observed to be soluble in the buffer solution. The protected glycine (BOC-glycine) has no visible reaction with genipin as no yellow compounds were 

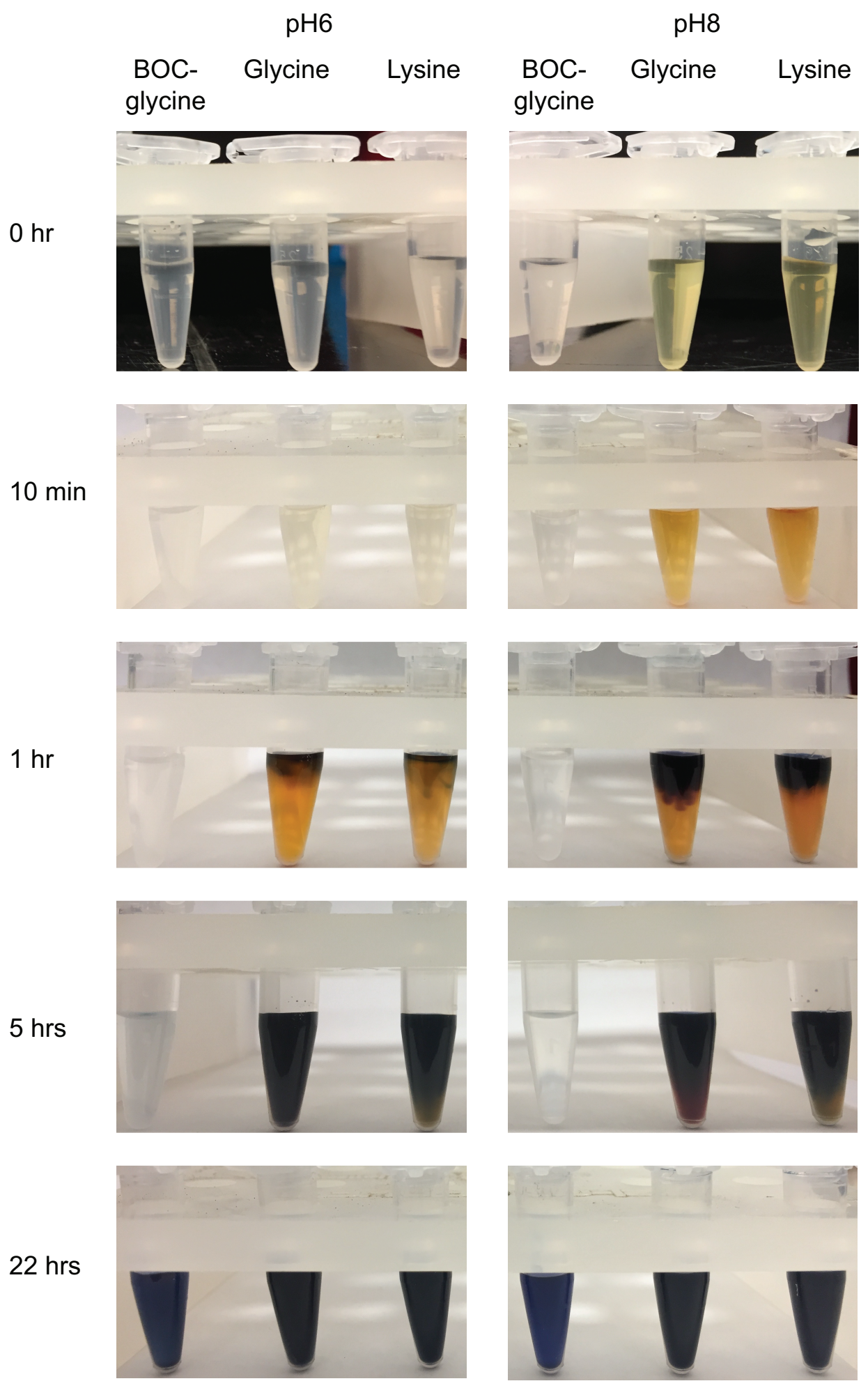

Figure 4.4 Reaction progression of genipin with BOC-glycine, glycine and lysine amino acids in $\mathrm{pH} 6$ phosphate buffer and in $\mathrm{pH} 8$ borate buffer. The formation of colored intermediates and the final water-soluble blue pigment. 
observed. Different buffer solutions revealed that the reaction was much slower in $\mathrm{pH} 6$ phosphate buffer than at $\mathrm{pH} 8$ borate buffer, Figure 4.4. Mi and coworkers showed that $\mathrm{pH}$ affects the extent of cross-linking of chitosan with genipin. They observed that the extent of cross-linking was highest at $\mathrm{pH} 7.4(\sim 96 \%)$ whereas $1 \%$ cross-linking was observed at $\mathrm{pH} 13 .{ }^{169}$ Genipin cross-linking with macromolecules such as collagen, ${ }^{166}$ silk-fibroin, ${ }^{172}$ chitosan ${ }^{173}$ and minielastin ${ }^{15}$ show that the stiffness and swelling properties of the cross-linked materials were found to be dependent on the extent of cross-linking and modulated by $\mathrm{pH} .{ }^{169}$

The cross-linking of minielastin $24 \mathrm{x}^{\prime}$ was done using the protocol published by Muiznieks. ${ }^{171}$ Recall that, prior to cross-linking, the liquid-liquid phase transition of tropoelastin must occur. Therefore, it is essential to induce coacervation which is done by increasing the salt concentration. Coacervation is depicted by the onset of turbidity as

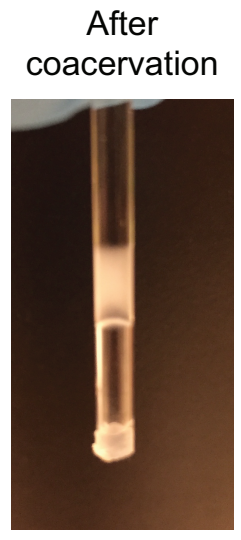

a

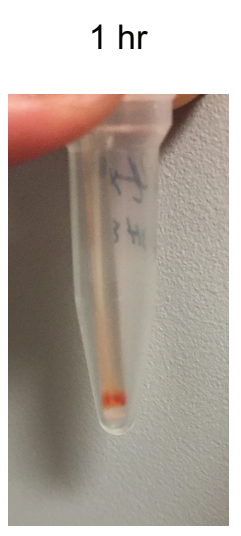

b
$2 \mathrm{hrs}$

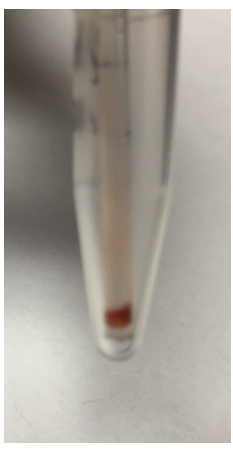

C
$6 \mathrm{hrs}$

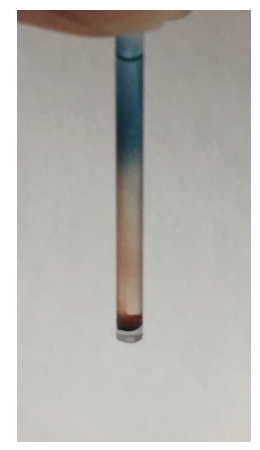

d
45 hrs

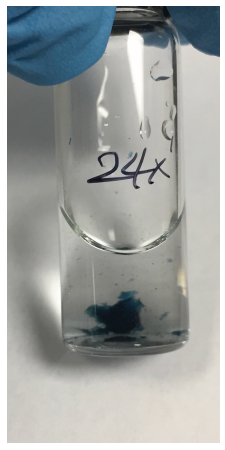

e

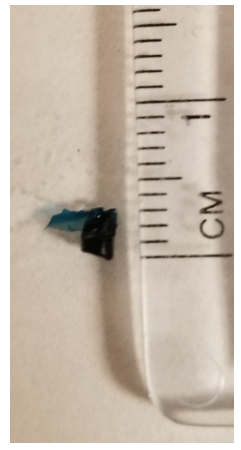

f

Figure 4.5 Progression of the cross-linking process. (a) following addition of $\mathrm{NaCl}$, the solution turns turbid, (b - c) following addition of genipin and centrifugation, the coacervate appears at the bottom of the $3 \mathrm{~mm}$ diameter tube as a reddish band, (d) after 6 hours the solution turned blue. (e) The insoluble, cross-linked $24 \mathrm{x}^{\prime}$ removed from the tube f) with length $\sim 3 \mathrm{~mm}$. 
shown in Figure 4.5. The denser particles i.e., the coacervates, were separated by centrifugation. The reaction progress can be observed with the formation of reddish color at the bottom of the tube. After 6 hours, the emergence of the blue pigment originating from the top of the tube was observed with the amino acid solutions, Figure 4.4. The blue pigment is the oxidation product from genipin cross-linking. ${ }^{164}$

An insoluble, cross-linked product of $24 x^{\prime}$ was successfully synthesized. Like tropoelastin ${ }^{174}$ and shorter minielastins with longer cross-link modules that have been previously studied, ${ }^{15} 24 \mathrm{x}^{\prime}$ also produces an insoluble cross-linked product. Future work in this project involves the measurement of its mechanical properties such as the Young's modulus and the swelling ratio of the cross-linked product. Moreover, the goal is to study the changes in protein dynamics that occur when the cross-linked material is formed and to relate the protein's sequence and length to its mechanical properties. 


\section{CHAPTER 5. CONCLUSION}

In this work, NMR spectroscopy was used to investigate the structure and dynamics of designed elastin mimetics, minielastins. Full chemical shift assignments of four minielastin constructs were reported. The hydrophobic module $20^{\prime}$ and $24^{\prime}$ of minielastins were shown to be highly disordered using secondary shift analysis of different nuclei. The secondary shift analysis as well as NOE intensities have shown trends not consistent with presence of secondary structures. Moreover, the secondary shift analysis for the cross-link module shows $\alpha$-helical propensity that is also supported by NOEs.

High polymer disorder is necessary for protein function. Unlike typical IDPs that are abundant in charged residues and depleted with hydrophobic residues, elastin and elastin-like proteins are rich in PG residues. Using PFG NMR, minielastins were shown to be more compact than typical IDPs but less compact than folded proteins. The effect of temperature and pressure on the hydrodynamic radii of minielastins were also investigated and found that increase in pressure and temperature decreases the hydrodynamic radii, however, the conformational or population changes were not explored. The effect of the hydrophobic repeats, VPGVGG and APGVGV, to coacervation temperature with each repeat was shown to decrease $T_{c}$ by 1.7 and $1.5{ }^{\circ} \mathrm{C}$, respectively. 
In Chapter 3, the timescales and amplitudes of motions were determined using spin relaxation measurements and spectral density modeling. The observed difference in secondary shifts between the hydrophobic and cross-link modules from chapter 1 was reflected on the spin relaxation analysis with the cross-link region having longer intermediate correlation times. The conservation of high degree of disorder from the soluble monomer to the cross-linked material was hypothesized. The order parameter, $\mathrm{S}^{2}$, was found to be less than 0.2 for all residues indicating a high degree of backbone disorder. This is consistent with the previously reported value of $\mathrm{S}<0.1$ for the mature bovine ligamentum nuchae using solid state NMR. ${ }^{117}$ The dynamics of other minielastin construct is on the works and this will allow us to further understand the difference in flexibility of $20^{\prime}$ and $24^{\prime}$ as well as the dynamical changes associated with protein length.

The study on the mechanical properties in relation to minielastin length and sequence has yet be explored. Double-labelled $\left({ }^{15} \mathrm{~N},{ }^{13} \mathrm{C}\right)$ cross-linked minielastin materials can be used to investigate the conformational changes or lack thereof, associated with stretch and recoil. It has been previously shown that the change in water ordering is negative upon recoil suggesting that the entropy of water plays a major role in elastic recoil. Furthermore, changes in backbone dynamics between the monomer and the cross-linked products can be directly compared.

This work takes a step closer to understanding the entropic mechanism of elastic recoil. The conserved disorder from the monomer to the cross-linked material implies that disorder is necessary for elastin function. Furthermore, understanding the relationship between protein sequence and length to elastic properties will help develop function-specific, elastin -based biomaterials. 


\section{REFERENCES}

1. Weis-Fogh, T.; Anderson, S. O., New molecular model for the long-range elasticity of elastin. Nature 1970, 227 (5259), 718-21.

2. Smith, D. W.; Weissman, N.; Carnes, W. H., Cardiovascular studies on copper deficient swine. XII. Partial purification of a soluble protein resembling elastin. Biochem Biophys Res Commun 1968, 31 (3), 309-15.

3. Indik, Z.; Yeh, H.; Ornstein-Goldstein, N.; Sheppard, P.; Anderson, N.; Rosenbloom, J. C.; Peltonen, L.; Rosenbloom, J., Alternative splicing of human elastin mRNA indicated by sequence analysis of cloned genomic and complementary DNA. Proc Natl Acad Sci U S A 1987, 84 (16), 5680-4.

4. Bashir, M. M.; Indik, Z.; Yeh, H.; Ornsteingoldstein, N.; Rosenbloom, J. C.; Abrams, W.; Fazio, M.; Uitto, J.; Rosenbloom, J., Characterization of the Complete Human Elastin Gene - Delineation of Unusual Features in the 5'-Flanking Region. $J$ Biol Chem 1989, 264 (15), 8887-8891.

5. Heinz, A.; Ruttkies, C. K. H.; Jahreis, G.; Schrader, C. U.; Wichapong, K.; Sippl, W.; Keeley, F. W.; Neubert, R. H. H.; Schmelzer, C. E. H., In vitro cross-linking of elastin peptides and molecular characterization of the resultant biomaterials. Bba-Gen Subjects 2013, 1830 (4), 2994-3004.

6. Urry, D. W.; Cunningham, W. D.; Ohnishi, T., Studies on the conformation and interactions of elastin. Proton magnetic resonance of the repeating pentapeptide. Biochemistry-Us 1974, 13 (3), 609-16.

7. Foster, J. A.; Bruenger, E.; Rubin, L.; Imberman, M.; Kagan, H.; Mecham, R.; Franzblau, C., Circular-Dichroism Studies of an Elastin Crosslinked Peptide. Biopolymers 1976, 15 (5), 833-841.

8. Muiznieks, L. D.; Jensen, S. A.; Weiss, A. S., Structural changes and facilitated association of tropoelastin. Arch Biochem Biophys 2003, 410 (2), 317-323.

9. Vrhovski, B.; Weiss, A. S., Biochemistry of tropoelastin. Eur J Biochem 1998, $258(1), 1-18$.

10. Urry, D. W., Elastic Biomolecular Machines. Sci Am 1995, 272 (1), 64-69. 
11. Miao, M.; Cirulis, J. T.; Lee, S.; Keeley, F. W., Structural determinants of crosslinking and hydrophobic domains for self-assembly of elastin-like polypeptides. Biochemistry-Us 2005, 44 (43), 14367-14375.

12. Miao, M.; Bellingham, C. M.; Stahl, R. J.; Sitarz, E. E.; Lane, C. J.; Keeley, F. W., Sequence and structure determinants for the self-aggregation of recombinant polypeptides modeled after human elastin. J Biol Chem 2003, 278 (49), 48553-48562.

13. Bellingham, C. M.; Woodhouse, K. A.; Robson, P.; Rothstein, S. J.; Keeley, F. W., Self-aggregation characteristics of recombinantly expressed human elastin polypeptides. Bba-Protein Struct M 2001, 1550 (1), 6-19.

14. Bellingham, C. M.; Lillie, M. A.; Gosline, J. M.; Wright, G. M.; Starcher, B. C.; Bailey, A. J.; Woodhouse, K. A.; Keeley, F. W., Recombinant human elastin polypeptides self-assemble into biomaterials with elastin-like properties. Biopolymers 2003, 70 (4), 445-455.

15. Vieth, S.; Bellingham, C. M.; Keeley, E. W.; Hodge, S. M.; Rousseau, D., Microstructural and tensile properties of elastin-based polypeptides crosslinked with genipin and pyrroloquinoline quinone. Biopolymers 2007, 85 (3), 199-206.

16. Sattler, M.; Schleucher, J.; Griesinger, C., Heteronuclear multidimensional NMR experiments for the structure determination of proteins in solution employing pulsed field gradients. Prog Nucl Mag Res Sp 1999, 34 (2), 93-158.

17. Wishart, D. S.; Sykes, B. D.; Richards, F. M., The Chemical-Shift Index - a Fast and Simple Method for the Assignment of Protein Secondary Structure through NmrSpectroscopy. Biochemistry-Us 1992, 31 (6), 1647-1651.

18. Kjaergaard, M.; Brander, S.; Poulsen, F. M., Random coil chemical shift for intrinsically disordered proteins: effects of temperature and $\mathrm{pH}$. J Biomol Nmr 2011, 49 (2), 139-149.

19. Wishart, D. S.; Sykes, B. D., Chemical-Shifts as a Tool for Structure Determination. Method Enzymol 1994, 239, 363-392.

20. Urry, D. W., Entropic Elastic Processes in Protein Mechanisms .1. Elastic Structure Due to an Inverse Temperature Transition and Elasticity Due to Internal Chain Dynamics. J Protein Chem 1988, 7 (1), 1-34.

21. Luan, C. H.; Chang, D. K.; Parker, T. M.; Krishna, N. R.; Urry, D. W., BetaSpiral Conformations of the Elastomeric Polytetrapeptides, (Vpgg)N and (Ipgg)N, by 2d Nmr and Molecular Mechanics Studies. Int J Quantum Chem 1991, 40, 183-198. 
22. Tamburro, A. M.; Pepe, A.; Bochicchio, B., Localizing alpha-helices in human tropoelastin: Assembly of the elastin "puzzle". Biochemistry-Us 2006, 45 (31), 95189530 .

23. Culik, R. M.; Abaskharon, R. M.; Pazos, I. M.; Gai, F., Experimental validation of the role of trifluoroethanol as a nanocrowder. J Phys Chem B 2014, 118 (39), 1145561.

24. Bodkin, M. J.; Goodfellow, J. M., Hydrophobic solvation in aqueous trifluoroethanol solution. Biopolymers 1996, 39 (1), 43-50.

25. Sonnichsen, F. D.; Van Eyk, J. E.; Hodges, R. S.; Sykes, B. D., Effect of trifluoroethanol on protein secondary structure: an NMR and CD study using a synthetic actin peptide. Biochemistry-Us 1992, 31 (37), 8790-8.

26. Wuthrich, K.; Billeter, M.; Braun, W., Polypeptide Secondary Structure Determination by Nuclear Magnetic-Resonance Observation of Short Proton Proton Distances. J Mol Biol 1984, 180 (3), 715-740.

27. Ma, K.; Kan, L. S.; Wang, K., Polyproline II helix is a key structural motif of the elastic PEVK segment of titin. Biochemistry-Us 2001, 40 (12), 3427-3438.

28. Fiebig, K. M.; Schwalbe, H.; Buck, M.; Smith, L. J.; Dobson, C. M., Toward a description of the conformations of denatured states of proteins. Comparison of a random coil model with NMR measurements. J Phys Chem-Us 1996, 100 (7), 2661-2666.

29. Cierpicki, T.; Otlewski, J., Amide proton temperature coefficients as hydrogen bond indicators in proteins. J Biomol Nmr 2001, 21 (3), 249-261.

30. Greenland, K. N.; Carvajal, M. F. C. A.; Preston, J. M.; Ekblad, S.; Dean, W. L.; Chiang, J. Y.; Koder, R. L.; Wittebort, R. J., Order, Disorder, and Temperature-Driven Compaction in a Designed Elastin Protein. J Phys Chem B 2018, 122 (10), 2725-2736.

31. Delaglio, F.; Grzesiek, S.; Vuister, G. W.; Zhu, G.; Pfeifer, J.; Bax, A., Nmrpipe a Multidimensional Spectral Processing System Based on Unix Pipes. J Biomol Nmr 1995, 6 (3), 277-293.

32. Lee, W.; Tonelli, M.; Markley, J. L., NMRFAM-SPARKY: enhanced software for biomolecular NMR spectroscopy. Bioinformatics 2015, 31 (8), 1325-1327.

33. Goddard, T. D. a. K., D. G., SPARKY 3, https://www.cgl.ucsf.edu/home/sparky/.

34. Cavanagh, J.; Fairbrother, W. J.; Palmer, A. G., III; Skelton, N. J.; Cavanagh, J.; Fairbrother, W. J.; Palmer, A. G., III; Skelton, N. J., Experimental aspects of NMR spectroscopy. 1996; p 95-182. 
35. Kragelj, J.; Ozenne, V.; Blackledge, M.; Jensen, M. R., Conformational Propensities of Intrinsically Disordered Proteins from NMR Chemical Shifts. Chemphyschem 2013, 14 (13), 3034-3045.

36. Shen, Y.; Bax, A., SPARTA plus : a modest improvement in empirical NMR chemical shift prediction by means of an artificial neural network. J Biomol Nmr 2010, $48(1), 13-22$.

37. Camilloni, C.; De Simone, A.; Vranken, W. F.; Vendruscolo, M., Determination of Secondary Structure Populations in Disordered States of Proteins Using Nuclear Magnetic Resonance Chemical Shifts. Biochemistry-Us 2012, 51 (11), 2224-2231.

38. Reichheld, S. E.; Muiznieks, L. D.; Keeley, F. W.; Sharpe, S., Direct observation of structure and dynamics during phase separation of an elastomeric protein. Proc. Natl. Acad. Sci. U. S. A. 2017, 114 (22), E4408-E4415.

39. Merutka, G.; Dyson, H. J.; Wright, P. E., Random Coil H-1 Chemical-Shifts Obtained as a Function of Temperature and Trifluoroethanol Concentration for the Peptide Series Ggxgg. J Biomol Nmr 1995, 5 (1), 14-24.

40. Baxter, N. J.; Williamson, M. P., Temperature dependence of H-1 chemical shifts in proteins. J Biomol Nmr 1997, 9 (4), 359-369.

41. Andersen, N. H.; Neidigh, J. W.; Harris, S. M.; Lee, G. M.; Liu, Z. H.; Tong, H., Extracting information from the temperature gradients of polypeptide NH chemical shifts .1. The importance of conformational averaging. J Am Chem Soc 1997, 119 (36), 85478561.

42. Toonkool, P.; Jensen, S. A.; Maxwell, A. L.; Weiss, A. S., Hydrophobic domains of human tropoelastin interact in a context-dependent manner. $J$ Biol Chem 2001, 276 (48), 44575-44580.

43. Lopes, J. L. S.; Miles, A. J.; Whitmore, L.; Wallace, B. A., Distinct circular dichroism spectroscopic signatures of polyproline II and unordered secondary structures: Applications in secondary structure analyses. Protein Sci 2014, 23 (12), 1765-1772.

44. Whitmore, L.; Wallace, B. A., Protein secondary structure analyses from circular dichroism spectroscopy: Methods and reference databases. Biopolymers 2008, 89 (5), $392-400$.

45. Provencher, S. W.; Glockner, J., Estimation of Globular Protein Secondary Structure from Circular-Dichroism. Biochemistry-Us 1981, 20 (1), 33-37.

46. Sreerama, N.; Woody, R. W., A Self-Consistent Method for the Analysis of Protein Secondary Structure from Circular-Dichroism. Anal Biochem 1993, 209 (1), 3244. 
47. Vrhovski, B.; Jensen, S.; Weiss, A. S., Coacervation characteristics of recombinant human tropoelastin. Eur J Biochem 1997, 250 (1), 92-98.

48. He, D.; Chung, M.; Chan, E.; Alleyne, T.; Ha, K. C. H.; Miao, M.; Stahl, R. J.; Keeley, F. W.; Parkinson, J., Comparative genomics of elastin: Sequence analysis of a highly repetitive protein. Matrix Biol 2007, 26 (7), 524-540.

49. Uversky, V. N., Intrinsically Disordered Proteins and Their "Mysterious" (Meta)Physics. Frontiers in Physics 2019, 7 (10).

50. Fisher, C. K.; Stultz, C. M., Constructing ensembles for intrinsically disordered proteins. Curr Opin Struct Biol 2011, 21 (3), 426-31.

51. van der Lee, R.; Buljan, M.; Lang, B.; Weatheritt, R. J.; Daughdrill, G. W.; Dunker, A. K.; Fuxreiter, M.; Gough, J.; Gsponer, J.; Jones, D. T.; Kim, P. M.; Kriwacki, R. W.; Oldfield, C. J.; Pappu, R. V.; Tompa, P.; Uversky, V. N.; Wright, P. E.; Babu, M. M., Classification of intrinsically disordered regions and proteins. Chem Rev 2014, 114 (13), 6589-631.

52. Wilkins, D. K.; Grimshaw, S. B.; Receveur, V.; Dobson, C. M.; Jones, J. A.; Smith, L. J., Hydrodynamic radii of native and denatured proteins measured by pulse field gradient NMR techniques. Biochemistry-Us 1999, 38 (50), 16424-16431.

53. Li, N. K.; Garcia Quiroz, F.; Hall, C. K.; Chilkoti, A.; Yingling, Y. G., Molecular description of the LCST behavior of an elastin-like polypeptide. Biomacromolecules 2014, 15 (10), 3522-30.

54. Rauscher, S.; Baud, S.; Miao, M.; Keeley, F. W.; Pomes, R., Proline and glycine control protein self-organization into elastomeric or amyloid fibrils. Structure 2006, 14 (11), 1667-1676.

55. Kozel, B. A.; Wachi, H.; Davis, E. C.; Mecham, R. P., Domains in tropoelastin that mediate elastin deposition in vitro and in vivo. J Biol Chem 2003, 278 (20), 1849118498.

56. Muiznieks, L. D.; Keeley, F. W., Proline periodicity modulates the self-assembly properties of elastin-like polypeptides. $J$ Biol Chem 2010, 285 (51), 39779-89.

57. Uversky, V. N., Natively unfolded proteins: A point where biology waits for physics. Protein Sci 2002, 11 (4), 739-756.

58. Marsh, J. A.; Forman-Kay, J. D., Sequence Determinants of Compaction in Intrinsically Disordered Proteins. Biophys J 2010, 98 (10), 2383-2390. 
59. Tomasso, M. E.; Tarver, M. J.; Devarajan, D.; Whitten, S. T., Hydrodynamic Radii of Intrinsically Disordered Proteins Determined from Experimental Polyproline II Propensities. Plos Comput Biol 2016, 12 (1).

60. Flory, P. J., Principles of polymer chemistry. Cornell University Press: Ithaca, N.Y., 1953.

61. Flory, P. J., The Configuration of Real Polymer Chains. The Journal of Chemical Physics 1949, 17 (3), 303-310.

62. Gennes, P.-G. d., Scaling concepts in polymer physics. Cornell University Press: Ithaca, N.Y., 1979.

63. Hofmann, H.; Soranno, A.; Borgia, A.; Gast, K.; Nettels, D.; Schuler, B., Polymer scaling laws of unfolded and intrinsically disordered proteins quantified with singlemolecule spectroscopy. Proc Natl Acad Sci U S A 2012, 109 (40), 16155-60.

64. Chen, C. R.; Makhatadze, G. I., Molecular determinant of the effects of hydrostatic pressure on protein folding stability. Nature Communications 2017, 8 (1), 14561.

65. Roche, J.; Royer, C. A.; Roumestand, C., Exploring Protein Conformational Landscapes Using High-Pressure NMR. Methods Enzymol 2019, 614, 293-320.

66. Roche, J.; Royer, C. A., Lessons from pressure denaturation of proteins. $J R$ Soc Interface 2018, 15 (147).

67. Royer, C. A., Revisiting volume changes in pressure-induced protein unfolding. Bba-Protein Struct M 2002, 1595 (1-2), 201-209.

68. Royer, C. A., A discussion of the physical basis for the pressure unfolding of proteins. Progr Biotechnol 2002, 19, 17-25.

69. Frye, K. J.; Royer, C. A., Probing the contribution of internal cavities to the volume change of protein unfolding under pressure. Protein Sci 1998, 7 (10), 2217-2222.

70. Frye, K. J.; Royer, C. A., The role of cavities in the pressure denatured state of staphylococcal nuclease. Biophys $J$ 1998, 74 (2), A166-A166.

71. Chen, C. R.; Makhatadze, G. I., Molecular Determinants of Temperature Dependence of Protein Volume Change upon Unfolding. J Phys Chem B 2017, 121 (35), 8300-8310.

72. Kjaergaard, M.; Norholm, A. B.; Hendus-Altenburger, R.; Pedersen, S. F.; Poulsen, F. M.; Kragelund, B. B., Temperature-dependent structural changes in 
intrinsically disordered proteins: Formation of alpha-helices or loss of polyproline II? Protein Sci 2010, 19 (8), 1555-1564.

73. Langridge, T. D.; Tarver, M. J.; Whitten, S. T., Temperature effects on the hydrodynamic radius of the intrinsically disordered $\mathrm{N}$-terminal region of the $\mathrm{p} 53$ protein. Proteins 2014, 82 (4), 668-678.

74. Cole, J. L.; Lary, J. W.; T, P. M.; Laue, T. M., Analytical ultracentrifugation: sedimentation velocity and sedimentation equilibrium. Methods Cell Biol 2008, 84, 14379.

75. Toonkool, P.; Regan, D. G.; Kuchel, P. W.; Morris, M. B.; Weiss, A. S., Thermodynamic and hydrodynamic properties of human tropoelastin - Analytical ultracentrifuge and pulsed field-gradient spin-echo NMR studies. J Biol Chem 2001, 276 (30), 28042-28050.

76. Stejskal, E. O.; Tanner, J. E., Spin Diffusion Measurements: Spin Echoes in the Presence of a Time-Dependent Field Gradient. J Chem Phys 1965, 42 (1), 288-292.

77. Johnson, C. S., Diffusion ordered nuclear magnetic resonance spectroscopy: principles and applications. Prog Nucl Mag Res Sp 1999, 34 (3-4), 203-256.

78. Debelle, L.; Tamburro, A. M., Elastin: molecular description and function. Int $J$ Biochem Cell B 1999, 31 (2), 261-272.

79. Mithieux, S. M.; Weiss, A. S., Elastin. Fibrous Proteins: Coiled-Coils, Collagen and Elastomers 2005, 70, 437-+.

80. Urry, D. W.; Trapane, T. L.; Prasad, K. U., Phase-Structure Transitions of the Elastin Polypentapeptide Water-System within the Framework of Composition Temperature Studies. Biopolymers 1985, 24 (12), 2345-2356.

81. Keeley, F. W.; Bellingham, C. M.; Woodhouse, K. A., Elastin as a self-organizing biomaterial: use of recombinantly expressed human elastin polypeptides as a model for investigations of structure and self-assembly of elastin. Philos T Roy Soc B 2002, 357 (1418), 185-189.

82. Yeo, G. C.; Keeley, F. W.; Weiss, A. S., Coacervation of tropoelastin. Adv Colloid Interfac 2011, 167 (1-2), 94-103.

83. Urry, D. W.; Luan, C. H.; Parker, T. M.; Gowda, D. C.; Prasad, K. U.; Reid, M. C.; Safavy, A., Temperature of Polypeptide Inverse Temperature Transition Depends on Mean Residue Hydrophobicity. J Am Chem Soc 1991, 113 (11), 4346-4348. 
84. Wu, D. H.; Chen, A. D.; Johnson, C. S., An Improved Diffusion-Ordered Spectroscopy Experiment Incorporating Bipolar-Gradient Pulses. J Magn Reson Ser A 1995, 115 (2), 260-264.

85. Wang, J. H., Self-Diffusion Coefficients of Water. J Phys Chem 1965, 69 (12), $4412-4412$.

86. Altieri, A. S.; Hinton, D. P.; Byrd, R. A., Association of Biomolecular Systems via Pulsed Field Gradient NMR Self-Diffusion Measurements. J Am Chem Soc 1995, 117 (28), 7566-7567.

87. Price, W. S.; Tsuchiya, F.; Arata, Y., Lysozyme aggregation and solution properties studied using PGSE NMR diffusion measurements. J Am Chem Soc 1999, 121 (49), 11503-11512.

88. Cooper, J. R., IAPWS. http://www.viscopedia.com/viscositytables/substances/water/ 2008.

89. Smith, C. A., Estimation of Sedimentation Coefficients and Frictional Ratios of Globular-Proteins. Biochem Educ 1988, 16 (2), 104-106.

90. Fluegel, S.; Fischer, K.; McDaniel, J. R.; Chilkoti, A.; Schmidt, M., Chain stiffness of elastin-like polypeptides. Biomacromolecules 2010, 11 (11), 3216-8.

91. Lam, S. L.; Hsu, V. L., NMR identification of left-handed polyproline type II helices. Biopolymers 2003, 69 (2), 270-281.

92. Li, B.; Alonso, D. O.; Daggett, V., The molecular basis for the inverse temperature transition of elastin. J Mol Biol 2001, 305 (3), 581-92.

93. Meyer, D. E.; Chilkoti, A., Quantification of the effects of chain length and concentration on the thermal behavior of elastin-like polypeptides. Biomacromolecules 2004, 5 (3), 846-51.

94. Li, N. K.; Roberts, S.; Quiroz, F. G.; Chilkoti, A.; Yingling, Y. G., Sequence Directionality Dramatically Affects LCST Behavior of Elastin-Like Polypeptides. Biomacromolecules 2018, 19 (7), 2496-2505.

95. Roche, J.; Ying, J. F.; Maltsev, A. S.; Bax, A., Impact of Hydrostatic Pressure on an Intrinsically Disordered Protein: A High-Pressure NMR Study of alpha-Synuclein. Chembiochem 2013, 14 (14), 1754-1761.

96. Chalikian, T. V., Volumetric properties of proteins. Annu Rev Bioph Biom 2003, $32,207-235$. 
97. Akasaka, K., Probing conformational fluctuation of proteins by pressure perturbation. Chem Rev 2006, 106 (5), 1814-1835.

98. Akasaka, K.; Li, H., Low-lying excited states of proteins revealed from nonlinear pressure shifts in 1H and 15N NMR. Biochemistry-Us 2001, 40 (30), 8665-71.

99. Beck Erlach, M.; Kalbitzer, H. R.; Winter, R.; Kremer, W., The pressure and temperature perturbation approach reveals a whole variety of conformational substates of amyloidogenic hIAPP monitored by 2D NMR spectroscopy. Biophys Chem 2019, 254, 106239.

100. Li, H.; Yamada, H.; Akasaka, K., Effect of pressure on individual hydrogen bonds in proteins. Basic pancreatic trypsin inhibitor. Biochemistry-Us 1998, 37 (5), 1167-73.

101. Erlach, M. B.; Koehler, J.; Munte, C. E.; Kremer, W.; Crusca, E.; Kainosho, M.; Kalbitzer, H. R., Pressure dependence of side chain(1)H and(15)N-chemical shifts in the model peptides Ac-Gly-Gly-Xxx-Ala-NH2. J Biomol Nmr 2020.

102. Erlach, M. B.; Koehler, J.; Crusca, E., Jr.; Kremer, W.; Munte, C. E.; Kalbitzer, H. R., Pressure dependence of backbone chemical shifts in the model peptides Ac-GlyGly-Xxx-Ala-NH2. J Biomol Nmr 2016, 65 (2), 65-77.

103. Koehler, J.; Erlach, M. B.; Crusca, E.; Kremer, W.; Munte, C. E.; Kalbitzer, H. R., Pressure Dependence of N-15 Chemical Shifts in Model Peptides Ac-Gly-Gly-X-AlaNH2. Materials 2012, 5 (10), 1774-1786.

104. Kamatari, Y. O.; Yamada, H.; Akasaka, K.; Jones, J. A.; Dobson, C. M.; Smith, L. J., Response of native and denatured hen lysozyme to high pressure studied by (15)N/(1)H NMR spectroscopy. Eur J Biochem 2001, 268 (6), 1782-93.

105. Kundrot, C. E.; Richards, F. M., Crystal structure of hen egg-white lysozyme at a hydrostatic pressure of 1000 atmospheres. J Mol Biol 1987, 193 (1), 157-70.

106. Ramanujam, V.; Alderson, T. R.; Pritisanac, I.; Ying, J. F.; Bax, A., Protein structural changes characterized by high-pressure, pulsed field gradient diffusion NMR spectroscopy. J Magn Reson 2020, 312.

107. Cinar, H.; Cinar, S.; Chan, H. S.; Winter, R., Pressure-Induced Dissolution and Reentrant Formation of Condensed, Liquid-Liquid Phase-Separated Elastomeric alphaElastin. Chem-Eur J 2018, 24 (33), 8286-8291.

108. Williamson, M. P.; Kitahara, R., Characterization of low-lying excited states of proteins by high-pressure NMR. Biochim Biophys Acta Proteins Proteom 2019, 1867 (3), 350-358. 
109. Orekhov, V. Y.; Dubovskii, P. V.; Yamada, H.; Akasaka, K.; Arseniev, A. S., Pressure effect on the dynamics of an isolated alpha-helix studied by $15 \mathrm{~N}-1 \mathrm{H}$ NMR relaxation. J Biomol Nmr 2000, 17 (3), 257-63.

110. Gosline, J. M., The elastic properties of rubber-like proteins and highly extensible tissues. Symp Soc Exp Biol 1980, 34, 332-57.

111. Brown-Augsburger, P.; Tisdale, C.; Broekelmann, T.; Sloan, C.; Mecham, R. P., Identification of an elastin cross-linking domain that joins three peptide chains. Possible role in nucleated assembly. J Biol Chem 1995, 270 (30), 17778-83.

112. Tarakanova, A.; Yeo, G. C.; Baldock, C.; Weiss, A. S.; Buehler, M. J., Tropoelastin is a Flexible Molecule that Retains its Canonical Shape. Macromol Biosci 2019, 19 (3).

113. Tarakanova, A.; Huang, W.; Weiss, A. S.; Kaplan, D. L.; Buehler, M. J., Computational smart polymer design based on elastin protein mutability. Biomaterials 2017, 127, 49-60.

114. Schraeder, C. U.; Heinz, A.; Majovsky, P.; Mayack, B. K.; Brinckmann, J.; Sippl, W.; Schmelzer, C. E. H., Elastin is heterogeneously cross-linked. J Biol Chem 2018, 293 (39), 15107-15119.

115. Hedtke, T.; Schrader, C. U.; Heinz, A.; Hoehenwarter, W.; Brinckmann, J.; Groth, T.; Schmelzer, C. E. H., A comprehensive map of human elastin cross-linking during elastogenesis. Febs J 2019, 286 (18), 3594-3610.

116. Ozsvar, J.; Tarakanova, A.; Wang, R.; Buehler, M. J.; Weiss, A. S., Allysine modifications perturb tropoelastin structure and mobility on a local and global scale. Matrix Biology Plus 2019, 2, 100002.

117. Pometun, M. S.; Chekmenev, E. Y.; Wittebort, R. J., Quantitative observation of backbone disorder in native elastin. J Biol Chem 2004, 279 (9), 7982.

118. Williamson, M. P., Drawing Single NMR Spins and Understanding Relaxation. 2019, 14 (5), 1934578X19849790.

119. Peng, J. W.; Wagner, G., Mapping of the Spectral Densities of N-H Bond Motions in Eglin-C Using Heteronuclear Relaxation Experiments. Biochemistry-Us 1992, 31 (36), 8571-8586.

120. Farrow, N. A.; Zhang, O. W.; Formankay, J. D.; Kay, L. E., Comparison of the Backbone Dynamics of a Folded and an Unfolded Sh3 Domain Existing in Equilibrium in Aqueous Buffer. Biochemistry-Us 1995, 34 (3), 868-878. 
121. Gill, M. L.; Byrd, R. A.; Palmer, A. G., Dynamics of GCN4 facilitate DNA interaction: a model-free analysis of an intrinsically disordered region. Phys Chem Chem Phys 2016, 18 (8), 5839-5849.

122. Farrow, N. A.; Muhandiram, R.; Singer, A. U.; Pascal, S. M.; Kay, C. M.; Gish, G.; Shoelson, S. E.; Pawson, T.; Forman-Kay, J. D.; Kay, L. E., Backbone dynamics of a free and phosphopeptide-complexed Src homology 2 domain studied by $15 \mathrm{~N}$ NMR relaxation. Biochemistry-Us 1994, 33 (19), 5984-6003.

123. Ochsenbein, F.; Guerois, R.; Neumann, J. M.; Sanson, A.; Guittet, E.; van Heijenoort, C., N-15 NMR relaxation as a probe for helical intrinsic propensity: The case of the unfolded D2 domain of annexin I. J Biomol Nmr 2001, 19 (1), 3-18.

124. Lipari, G.; Szabo, A., Model-Free Approach to the Interpretation of Nuclear Magnetic-Resonance Relaxation in Macromolecules .2. Analysis of Experimental Results. J Am Chem Soc 1982, 104 (17), 4559-4570.

125. Lipari, G.; Szabo, A., Model-Free Approach to the Interpretation of Nuclear Magnetic-Resonance Relaxation in Macromolecules .1. Theory and Range of Validity. $J$ Am Chem Soc 1982, 104 (17), 4546-4559.

126. Yao, S.; Babon, J. J.; Norton, R. S., Protein effective rotational correlation times from translational self-diffusion coefficients measured by PFG-NMR. Biophys Chem 2008, $136(2-3), 145-51$.

127. Clore, G. M.; Szabo, A.; Bax, A.; Kay, L. E.; Driscoll, P. C.; Gronenborn, A. M., Deviations from the simple two-parameter model-free approach to the interpretation of nitrogen-15 nuclear magnetic relaxation of proteins. J Am Chem Soc 1990, 112 (12), 4989-4991.

128. Salvi, N.; Abyzov, A.; Blackledge, M., Solvent-dependent segmental dynamics in intrinsically disordered proteins. Sci Adv 2019, 5 (6), eaax2348.

129. Salvi, N.; Abyzov, A.; Blackledge, M., Atomic resolution conformational dynamics of intrinsically disordered proteins from NMR spin relaxation. Prog Nucl Mag Res $S p$ 2017, 102, 43-60.

130. Ochsenbein, F.; Neumann, J. M.; Guittet, E.; van Heijenoort, C., Dynamical characterization of residual and non-native structures in a partially folded protein by (15)N NMR relaxation using a model based on a distribution of correlation times. Protein Sci 2002, 11 (4), 957-64.

131. Buevich, A. V.; Baum, J., Dynamics of Unfolded Proteins: Incorporation of Distributions of Correlation Times in the Model Free Analysis of NMR Relaxation Data. J Am Chem Soc 1999, 121 (37), 8671-8672. 
132. Modig, K.; Poulsen, F. M., Model-independent interpretation of NMR relaxation data for unfolded proteins: the acid-denatured state of ACBP. J Biomol Nmr 2008, 42 (3), 163-177.

133. Adamski, W.; Salvi, N.; Maurin, D.; Magnat, J.; Milles, S.; Jensen, M. R.; Abyzov, A.; Moreau, C. J.; Blackledge, M., A Unified Description of Intrinsically Disordered Protein Dynamics under Physiological Conditions Using NMR Spectroscopy. J Am Chem Soc 2019, 141 (44), 17817-17829.

134. Chakrabarti, K. S.; Ban, D.; Pratihar, S.; Reddy, J. G.; Becker, S.; Griesinger, C.; Lee, D., High-power H-1 composite pulse decoupling provides artifact free exchangemediated saturation transfer (EST) experiments. J Magn Reson 2016, 269, 65-69.

135. Carneiro, M. G.; Reddy, J. G.; Griesinger, C.; Lee, D., Speeding-up exchangemediated saturation transfer experiments by Fourier transform. J Biomol Nmr 2015, 63 (3), 237-244.

136. Gu, Q.; Zhu, H.; Li, J.; Li, X.; Hao, J.; Wallace, G. G.; Zhou, Q., Threedimensional bioprinting speeds up smart regenerative medicine. Natl Sci Rev 2016, 3 (3), $331-344$.

137. Farrow, N. A.; Zhang, O.; Szabo, A.; Torchia, D. A.; Kay, L. E., Spectral density function mapping using $15 \mathrm{~N}$ relaxation data exclusively. J Biomol Nmr 1995, 6 (2), 15362 .

138. Kadeřávek, P.; Zapletal, V.; Rabatinová, A.; Krásný, L.; Sklenáŕ, V.; Žídek, L., Spectral density mapping protocols for analysis of molecular motions in disordered proteins. J Biomol Nmr 2014, 58 (3), 193-207.

139. Palmer, A. G.; Rance, M.; Wright, P. E., Intramolecular Motions of a Zinc Finger DNA-Binding Domain from Xfin Characterized by Proton-Detected Natural Abundance C-12 Heteronuclear Nmr-Spectroscopy. J Am Chem Soc 1991, 113 (12), 4371-4380.

140. Zhang, X.; Perugini, M. A.; Yao, S.; Adda, C. G.; Murphy, V. J.; Low, A.; Anders, R. F.; Norton, R. S., Solution conformation, backbone dynamics and lipid interactions of the intrinsically unstructured malaria surface protein MSP2. J Mol Biol 2008, $379(1), 105-21$.

141. Bertini, I.; Gupta, Y. K.; Luchinat, C.; Parigi, G.; Schlorb, C.; Schwalbe, H., NMR spectroscopic detection of protein protons and longitudinal relaxation rates between 0.01 and $50 \mathrm{MHz}$. Angew Chem Int Ed Engl 2005, 44 (15), 2223-5.

142. Brady, J. P.; Farber, P. J.; Sekhar, A.; Lin, Y. H.; Huang, R.; Bah, A.; Nott, T. J.; Chan, H. S.; Baldwin, A. J.; Forman-Kay, J. D.; Kay, L. E., Structural and hydrodynamic properties of an intrinsically disordered region of a germ cell-specific protein on phase separation. Proc Natl Acad Sci U S A 2017, 114 (39), E8194-E8203. 
143. Khan, S. N.; Charlier, C.; Augustyniak, R.; Salvi, N.; Dejean, V.; Bodenhausen, G.; Lequin, O.; Pelupessy, P.; Ferrage, F., Distribution of Pico- and Nanosecond Motions in Disordered Proteins from Nuclear Spin Relaxation. Biophys J 2015, 109 (5), 988-999.

144. Eliezer, D.; Yao, J.; Dyson, H. J.; Wright, P. E., Structural and dynamic characterization of partially folded states of apomyoglobin and implications for protein folding. Nat Struct Biol 1998, 5 (2), 148-155.

145. Abyzov, A.; Salvi, N.; Schneider, R.; Maurin, D.; Ruigrok, R. W. H.; Jensen, M. R.; Blackledge, M., Identification of Dynamic Modes in an Intrinsically Disordered Protein Using Temperature-Dependent NMR Relaxation. J Am Chem Soc 2016, 138 (19), 6240-6251.

146. Frank, M. K.; Clore, G. M.; Gronenborn, A. M., Structural and Dynamic Characterization of the Urea Denatured State of the Immunoglobulin Binding Domain of Streptococcal Protein-G by Multidimensional Heteronuclear Nmr-Spectroscopy. Protein Sci 1995, 4 (12), 2605-2615.

147. Martin, E. W.; Holehouse, A. S.; Grace, C. R.; Hughes, A.; Pappu, R. V.; Mittag, T., Sequence Determinants of the Conformational Properties of an Intrinsically Disordered Protein Prior to and upon Multisite Phosphorylation. J Am Chem Soc 2016, 138 (47), 15323-15335.

148. Sterckx, Y. G. J.; Volkov, A. N.; Vranken, W. F.; Kragelj, J.; Jensen, M. R.; Buts, L.; Garcia-Pino, A.; Jove, T.; Van Melderen, L.; Blackledge, M.; van Nuland, N. A. J.; Loris, R., Small-Angle X-Ray Scattering- and Nuclear Magnetic Resonance-Derived Conformational Ensemble of the Highly Flexible Antitoxin PaaA2. Structure 2014, 22 (6), 854-865.

149. Gotte, L.; Stern, P.; Elsden, D. F.; Partridge, S. M., The chemistry of connective tissues. 8. The composition of elastin from three bovine tissues. Biochem J 1963, 87, 34451.

150. Hinek, A.; Rabinovitch, M., 67-Kd Elastin-Binding Protein Is a Protective Companion of Extracellular Insoluble Elastin and Intracellular Tropoelastin. J Cell Biol 1994, $126(2), 563-574$.

151. Partridge, S. M.; Elsden, D. F.; Thomas, J., Constitution of the cross-linkages in elastin. Nature 1963, 197, 1297-8.

152. Thomas, J.; Elsden, D. F.; Partridge, S. M., Partial Structure of Two Major Degradation Products from the Cross-Linkages in Elastin. Nature 1963, 200, 651-2. 
153. Mecham, R. P., Methods in elastic tissue biology: Elastin isolation and purification. Methods 2008, 45 (1), 32-41.

154. Baldock, C.; Oberhauser, A. F.; Ma, L. A.; Lammie, D.; Siegler, V.; Mithieux, S. M.; Tu, Y. D.; Chow, J. Y. H.; Suleman, F.; Malfois, M.; Rogers, S.; Guo, L. A.; Irving, T. C.; Wess, T. J.; Weiss, A. S., Shape of tropoelastin, the highly extensible protein that controls human tissue elasticity. Proc. Natl. Acad. Sci. U. S. A. 2011, 108 (11), 43224327.

155. Schmelzer, C. E. H.; Hedtke, T.; Heinz, A., Unique molecular networks: Formation and role of elastin cross-links. Iubmb Life 2019.

156. Zhang, Y. N.; Avery, R. K.; Vallmajo-Martin, Q.; Assmann, A.; Vegh, A.; Memic, A.; Olsen, B. D.; Annabi, N.; Khademhosseini, A., A Highly Elastic and Rapidly Crosslinkable Elastin-Like Polypeptide-Based Hydrogel for Biomedical Applications. Adv Funct Mater 2015, 25 (30), 4814-4826.

157. Wise, S. G.; Mithieux, S. M.; Raftery, M. J.; Weiss, A. S., Specificity in the coacervation of tropoelastin: solvent exposed lysines. J Struct Biol 2005, 149 (3), 273 281.

158. Lucero, H. A.; Kagan, H. M., Lysyl oxidase: an oxidative enzyme and effector of cell function. Cell Mol Life Sci 2006, 63 (19-20), 2304-2316.

159. Shah, M. A.; Bergethon, P. R.; Boak, A. M.; Gallop, P. M.; Kagan, H. M., Oxidation of Peptidyl Lysine by Copper-Complexes of Pyrroloquinoline Quinone and Other Quinones - a Model for Oxidative Pathochemistry. Biochim Biophys Acta 1992, 1159 (3), 311-318.

160. Sung, H. W.; Huang, R. N.; Huang, L. L. H.; Tsai, C. C., In vitro evaluation of cytotoxicity of a naturally occurring cross-linking reagent for biological tissue fixation. $J$ Biomat Sci-Polym E 1999, 10 (1), 63-78.

161. Fujikawa, S.; Nakamura, S.; Koga, K., Genipin, a New Type of Protein Crosslinking Reagent from Gardenia Fruits. Agr Biol Chem Tokyo 1988, 52 (3), 869-870.

162. Butler, M. F.; Ng, Y.-F.; Pudney, P. D. A., Mechanism and kinetics of the crosslinking reaction between biopolymers containing primary amine groups and genipin. Journal of Polymer Science Part A: Polymer Chemistry 2003, 41 (24), 3941-3953.

163. Touyama, R.; Takeda, Y.; Inoue, K.; Kawamura, I.; Yatsuzuka, M.; Ikumoto, T.; Shingu, T.; Yokoi, T.; Inouye, H., Studies on the Blue Pigments Produced from Genipin and Methylamine .1. Structures of the Brownish-Red Pigments, Intermediates Leading to the Blue Pigments. Chem Pharm Bull 1994, 42 (3), 668-673. 
164. Touyama, R.; Inoue, K.; Takeda, Y.; Yatsuzuka, M.; Ikumoto, T.; Moritome, N.; Shingu, T.; Yokoi, T.; Inouye, H., Studies on the Blue Pigments Produced from Genipin and Methylamine .2. On the Formation Mechanisms of Brownish-Red Intermediates Leading to the Blue Pigment Formation. Chem Pharm Bull 1994, 42 (8), 1571-1578.

165. Silva, S. S.; Maniglio, D.; Motta, A.; Mano, J. F.; Reis, R. L.; Migliaresi, C., Genipin-modified silk-fibroin nanometric nets. Macromol Biosci 2008, 8 (8), 766-774.

166. Sundararaghavan, H. G.; Monteiro, G. A.; Lapin, N. A.; Chabal, Y. J.; Miksan, J. R.; Shreiber, D. I., Genipin-induced changes in collagen gels: Correlation of mechanical properties to fluorescence. J Biomed Mater Res A 2008, 87a (2), 308-320.

167. Chang, W. H.; Chang, Y.; Lai, P. H.; Sung, H. W., A genipin-crosslinked gelatin membrane as wound-dressing material: in vitro and in vivo studies. J Biomat Sci-Polym E 2003, 14 (5), 481-495.

168. Sung, H. W.; Chang, W. H.; Ma, C. Y.; Lee, M. H., Crosslinking of biological tissues using genipin and/or carbodiimide. J Biomed Mater Res A 2003, 64 a (3), 427-438.

169. Mi, F.-L.; Shyu, S.-S.; Peng, C.-K., Characterization of ring-opening polymerization of genipin and $\mathrm{pH}$-dependent cross-linking reactions between chitosan and genipin. Journal of Polymer Science Part A: Polymer Chemistry 2005, 43 (10), 19852000 .

170. Di Tommaso, S.; David, H.; Gomar, J.; Leroy, F.; Adamo, C., From iridoids to dyes: a theoretical study on genipin reactivity. $R s c A d v$ 2014, 4 (22), 11029-11038.

171. Muiznieks, L. D., Genipin Cross-Linking of Elastin and Elastin-Based Proteins. Methods Mol Biol 2018, 1798, 213-221.

172. Elliott, W. H.; Bonani, W.; Maniglio, D.; Motta, A.; Tan, W.; Migliaresi, C., Silk Hydrogels of Tunable Structure and Viscoelastic Properties Using Different Chronological Orders of Genipin and Physical Cross-Linking. Acs Appl Mater Inter 2015, 7 (22), 12099-12108.

173. Khurma, J. R.; Rohindra, D. R.; Nand, A. V., Swelling and thermal characteristics of genipin crosslinked chitosan and poly(vinyl pyrrolidone) hydrogels. Polym Bull 2005, 54 (3), 195-204.

174. Bedellhogan, D.; Trackman, P.; Abrams, W.; Rosenbloom, J.; Kagan, H., Oxidation, Cross-Linking, and Insolubilization of Recombinant Tropoelastin by Purified Lysyl Oxidase. J Biol Chem 1993, 268 (14), 10345-10350. 


\section{APPENDICES}

Appendix I. Chemical shift assignment of minielastin constructs

Appendix I-A. Chemical shift assignment of doubly labeled 24x'.

\begin{tabular}{cccccccccc}
\hline Residue & $\mathbf{N}$ & $\mathbf{H}^{\mathbf{N}}$ & $\mathbf{H} \boldsymbol{\alpha}$ & $\mathbf{H} \boldsymbol{\beta}$ & $\mathbf{H} \boldsymbol{\gamma}$ & $\mathbf{C} \boldsymbol{\alpha}$ & $\mathbf{C} \boldsymbol{\beta}$ & $\mathbf{C} \boldsymbol{\gamma}$ & $\mathbf{C O}$ \\
\hline $\mathbf{A}$ & 129.4 & 8.42 & 4.57 & 1.33 & - & 50.4 & 18.3 & - & 175.3 \\
$\mathbf{P}$ & 135.2 & - & 4.38 & 2.26 & $2.02 / 1.91$ & 63.1 & 32.0 & 27.4 & 177.6 \\
$\mathbf{G}$ & 109.1 & 8.43 & 3.92 & - & - & 45.2 & & - & 174.1 \\
$\mathbf{V}$ & 119.2 & 7.96 & 4.11 & 2.08 & 0.905 & 62.4 & 32.6 & 21.1 & 176.7 \\
$\mathbf{G}$ & 112.6 & 8.52 & 3.92 & - & - & 45.2 & & - & 173.7 \\
$\mathbf{V}$ & 119.1 & 7.92 & 4.10 & 2.00 & 0.868 & 61.7 & 32.9 & 20.3 & 175.5 \\
& & & & & & & & & \\
$\mathbf{D}$ & 123.8 & 8.41 & 4.58 & $2.73 / 2.63$ & - & 54.1 & 41.0 & - & 176.5 \\
$\mathbf{A}$ & 125.9 & 8.39 & 4.14 & 1.40 & - & 54.0 & 18.9 & - & 178.9 \\
$\mathbf{A}$ & 121.8 & 8.25 & 4.19 & 1.40 & - & 53.7 & 18.5 & - & 179.0 \\
$\mathbf{A}$ & 122.2 & 7.97 & 4.20 & 1.40 & - & 53.7 & 18.6 & - & 179.2 \\
$\mathbf{A}$ & 121.9 & 8.11 & 4.15 & 1.40 & - & 53.6 & 18.3 & - & 178.8 \\
$\mathbf{A}$ & 121.6 & 7.95 & 4.21 & 1.35 & - & 53.4 & 18.4 & - & 178.8 \\
$\mathbf{K}$ & 119.1 & 7.92 & 4.17 & 1.80 & $1.49 / 1.40$ & 57.1 & 32.7 & 25.0 & 177.0 \\
$\mathbf{A}$ & 123.0 & 7.93 & 4.22 & 1.39 & - & 52.8 & 18.9 & - & 177.8 \\
$\mathbf{A}$ & 122.1 & 7.96 & 4.20 & 1.40 & - & 52.8 & 18.6 & - & 177.7 \\
$\mathbf{K}$ & 119.2 & 7.96 & 4.17 & 1.66 & $1.32 / 1.24$ & 56.4 & 32.8 & 24.7 & 176.1 \\
$\mathbf{F}$ & 120.3 & 8.05 & 4.60 & $3.11 / 2.96$ & - & 57.1 & 39.7 & - & 174.8 \\
\hline
\end{tabular}


Appendix I-B. Chemical shift assignment of ${ }^{15} \mathrm{~N}$-labeled 20x'.

\begin{tabular}{|c|c|c|c|c|c|c|c|c|}
\hline Residue & $\mathbf{N}$ & $\mathbf{H}^{\mathbf{N}}$ & $\mathrm{H} \alpha$ & $\mathbf{H \beta}$ & $\mathbf{H} \gamma$ & $\mathrm{C} \alpha$ & $C \beta$ & $C \gamma$ \\
\hline $\mathrm{V}\left(1,20^{\prime}\right)$ & 121.3 & 8.04 & 4.41 & 2.07 & 0.91 & 59.8 & 32.7 & 21.1 \\
\hline$P\left(2,20^{\prime}\right)$ & 139.7 & - & 4.37 & $2.27 / 1.92$ & $1.93 / 1.92$ & 63.2 & 32.1 & 27.4 \\
\hline$G\left(3,20^{\prime}\right)$ & 109.3 & 8.39 & 3.93 & - & - & 45.2 & - & - \\
\hline$V\left(4,20^{\prime}\right)$ & 119.4 & 8.03 & 4.15 & 2.08 & 0.91 & 62.4 & 32.7 & 21.1 \\
\hline$G\left(5,20^{\prime}\right)$ & 112.8 & 8.60 & 3.95 & - & - & 45.2 & - & - \\
\hline$G\left(6,20^{\prime}\right)$ & 108.5 & 8.25 & 3.93 & - & - & 45.2 & - & - \\
\hline D $(1, \mathbf{x} 1)$ & 120.8 & 8.33 & 4.59 & 2.67 & - & 54.3 & 41.1 & - \\
\hline A $(2, \mathbf{x} 1)$ & 125.4 & 8.41 & 4.18 & 1.41 & - & 53.7 & 19.0 & - \\
\hline$A(3, x 1)$ & 122.1 & 8.19 & 4.20 & 1.41 & - & 52.8 & 18.6 & - \\
\hline$A(4, x 1)$ & 122.3 & 8.00 & 4.22 & 1.40 & - & 52.8 & 18.6 & - \\
\hline $\mathrm{A}(5, \mathbf{x} 1)$ & 122.0 & 8.07 & 4.19 & 1.41 & - & 53.5 & 18.6 & - \\
\hline A $(6, \mathbf{x} 1)$ & 121.9 & 7.98 & 4.21 & 1.43 & - & 52.8 & 18.6 & - \\
\hline$K(\mathbf{7}, \mathbf{x})$ & 119.1 & 7.90 & 4.16 & 1.82 & 1.43 & 57.1 & 32.9 & 25.0 \\
\hline A $(\mathbf{8}, \mathbf{x} 1)$ & 123.3 & 7.97 & 4.20 & 4.40 & - & 53.5 & 19.0 & - \\
\hline A $(\mathbf{9}, \mathbf{x} 1)$ & 122.3 & 8.00 & 4.22 & 1.40 & - & 52.8 & 18.7 & - \\
\hline $\mathrm{K}(\mathbf{1 0}, \mathbf{x})$ & 119.4 & 7.96 & 4.16 & 1.67 & 1.36 & 56.4 & 33.0 & 24.6 \\
\hline$F(11, x)$ & 120.4 & 8.06 & 4.63 & $3.12 / 2.98$ & - & 57.3 & 39.8 & - \\
\hline$A\left(1,24^{\prime}\right)$ & 129.5 & 8.42 & 4.59 & 1.35 & - & 50.4 & 18.2 & - \\
\hline$P\left(2,24^{\prime}\right)$ & 135.2 & - & 4.40 & $2.27 / 1.94$ & $2.02 / 1.99$ & 63.2 & 32.1 & 27.5 \\
\hline
\end{tabular}




\begin{tabular}{|c|c|c|c|c|c|c|c|c|}
\hline$G\left(3,24^{\prime}\right)$ & 109.2 & 8.43 & 3.93 & - & - & 45.2 & - & - \\
\hline$V\left(4,24^{\prime}\right)$ & 119.4 & 7.96 & 4.13 & 2.10 & 0.92 & 62.4 & 32.7 & 21.1 \\
\hline$G\left(5,24^{\prime}\right)$ & 112.7 & 8.52 & 3.94 & - & - & 45.2 & - & - \\
\hline$V\left(6,24^{\prime}\right)$ & 119.2 & 7.92 & 4.11 & 2.03 & 0.89 & 62.5 & 33.0 & 20.6 \\
\hline$D(1, \mathbf{x} 2)$ & 123.9 & 8.42 & 4.60 & $2.74 / 2.66$ & - & 54.3 & 41.1 & - \\
\hline$A(2, x 2)$ & 126.0 & 8.39 & 4.16 & 1.41 & - & 53.7 & 19.0 & - \\
\hline$A(3, x 2)$ & 121.9 & 8.26 & 4.20 & 1.43 & - & 52.8 & 18.6 & - \\
\hline$A(4, x 2)$ & 122.3 & 7.97 & 4.22 & 1.43 & - & 52.8 & 18.6 & - \\
\hline$A(5, x 2)$ & 122.0 & 8.12 & 4.17 & 1.41 & - & 53.7 & 18.6 & - \\
\hline$A(6, x 2)$ & 121.7 & 7.96 & 4.20 & 1.42 & - & 52.8 & 18.6 & - \\
\hline $\mathbf{K}(7, \mathbf{x})$ & 119.1 & 7.90 & 4.16 & 1.82 & 1.43 & 57.1 & 32.9 & 25.0 \\
\hline$A(8, x 2)$ & 123.1 & 7.94 & 4.19 & 1.40 & - & 53.5 & 19.0 & - \\
\hline$A(9, x 2)$ & 122.3 & 7.97 & 4.22 & 1.43 & - & 52.8 & 18.7 & - \\
\hline$K(10, x)$ & 119.4 & 7.96 & 4.16 & 1.67 & 1.36 & 56.4 & 33.0 & 24.6 \\
\hline$F(11, x)$ & 120.4 & 8.06 & 4.63 & $3.12 / 2.98$ & - & 57.3 & 39.8 & - \\
\hline
\end{tabular}


Appendix I-C. Chemical shift assignment of ${ }^{13} \mathrm{C}$ and ${ }^{15} \mathrm{~N}$-labeled 202424.

\begin{tabular}{lccccccccc}
\hline $\mathbf{R e s i d u e}$ & $\mathbf{N}$ & $\mathbf{H}^{\mathbf{N}}$ & $\mathbf{H} \boldsymbol{\alpha}$ & $\mathbf{H} \boldsymbol{\beta}$ & $\mathbf{H} \boldsymbol{\gamma}$ & $\mathbf{C} \boldsymbol{\alpha}$ & $\mathbf{C} \boldsymbol{\beta}$ & $\mathbf{C} \boldsymbol{\gamma}$ & $\mathbf{C O}$ \\
\hline $\mathbf{V ( 1 , 2 0 )}$ & 121.2 & 8.04 & 4.40 & 2.05 & 0.91 & 59.9 & 32.6 & 20.9 & 174.6 \\
$\mathbf{P ( 2 , 2 0 )}$ & 139.6 & - & 4.33 & $2.25 / 1.90$ & $2.03 / 2.26$ & 63.6 & 32.1 & 27.4 & 177.4 \\
$\mathbf{G ( 3 , 2 0 )}$ & 109.2 & 8.40 & 3.93 & - & - & 45.2 & - & - & 174.2 \\
$\mathbf{V ( 4 , 2 0 )}$ & 119.3 & 8.03 & 4.15 & 2.08 & 0.91 & 62.4 & 32.7 & 20.9 & 176.8 \\
$\mathbf{G ( 5 , 2 0 )}$ & 112.7 & 8.60 & 3.94 & - & - & 45.2 & - & - & 174.5 \\
$\mathbf{G ( 6 , 2 0 )}$ & 108.4 & 8.25 & 3.93 & - & - & 45.1 & - & - & 173.7 \\
& & & & & & & & & \\
$\mathbf{E ( 1 , X 1 )}$ & 121.4 & 8.60 & 4.19 & 1.99 & 2.28 & 57.8 & 29.7 & 36.3 & 177.4 \\
$\mathbf{A ( 2 , X 1 )}$ & 124.3 & 8.44 & 4.21 & 1.40 & - & 53.6 & 18.8 & - & 179.1 \\
$\mathbf{Q ( 3 , X 1 )}$ & 119.3 & 8.28 & 4.21 & 2.04 & 2.39 & 57.2 & 28.8 & 34.0 & 177.1 \\
$\mathbf{A ( 4 , X 1 )}$ & 124.2 & 8.21 & 4.20 & 1.41 & - & 53.8 & 18.7 & - & 179.0 \\
$\mathbf{A ( 5 , X 1 )}$ & 122.5 & 8.19 & 4.19 & 1.41 & - & 53.8 & 18.5 & - & 179.1 \\
$\mathbf{A ( 6 , X )}$ & 122.0 & 8.06 & 4.19 & 1.43 & - & 53.8 & 18.6 & - & 179.2 \\
$\mathbf{A ( 7 , X )}$ & 122.2 & 8.10 & 4.22 & 1.42 & - & 53.7 & 18.7 & - & 179.0 \\
$\mathbf{A ( 8 , X )}$ & 121.9 & 8.00 & 4.20 & 1.42 & - & 53.6 & 18.7 & - & 179.0 \\
$\mathbf{K}(\mathbf{9}, \mathbf{X})$ & 119.3 & 7.92 & 4.16 & 1.84 & $1.48 / 1.41$ & 57.4 & 32.7 & 24.9 & 177.1 \\
$\mathbf{A ( 1 0 , X )}$ & 122.9 & 7.96 & 4.18 & 1.40 & - & 53.0 & 18.5 & - & 178.1 \\
$\mathbf{A ( 1 1 , X )}$ & 122.0 & 7.98 & 4.20 & 1.36 & - & 53.0 & 18.6 & - & 178.0 \\
$\mathbf{K ( 1 2 , X )}$ & 119.3 & 7.92 & 4.16 & 1.64 & $1.26 / 1.14$ & 56.7 & 32.8 & 24.7 & 176.5 \\
$\mathbf{Y ( 1 3 , Y )}$ & 119.5 & 8.04 & 4.58 & $3.12 / 2.90$ & - & 57.8 & 38.8 & - & 176.4 \\
$\mathbf{G ( 1 4 , X )}$ & 110.1 & 8.21 & 3.92 & - & - & 45.3 & - & - & 174.0
\end{tabular}




\begin{tabular}{|c|c|c|c|c|c|c|c|c|c|}
\hline$V(15, X)$ & 119.0 & 8.03 & 4.14 & 2.11 & 0.93 & 62.3 & 32.8 & 20.9 & 176.8 \\
\hline$G(16, X)$ & 112.4 & 8.56 & 3.98 & - & - & 45.1 & - & - & 174.0 \\
\hline$T(17, X)$ & 115.3 & 7.99 & 4.60 & 4.21 & 1.23 & 60.1 & 69.9 & 21.6 & 173.3 \\
\hline$P(18, X)$ & 138.8 & - & 4.36 & $2.25 / 1.90$ & $2.03 / 2.26$ & 63.8 & 32.0 & 27.4 & 177.5 \\
\hline$A(19, X)$ & 123.4 & 8.34 & 4.20 & 1.36 & - & 53.3 & 18.5 & - & 178.5 \\
\hline$A(20, X)$ & 122.8 & 8.19 & 4.20 & 1.41 & - & 53.3 & 18.5 & - & 178.6 \\
\hline$A(21, X)$ & 122.5 & 8.12 & 4.22 & 1.40 & - & 53.2 & 18.8 & - & 178.7 \\
\hline $\mathbf{A}(22, X)$ & 122.6 & 8.15 & 4.21 & 1.40 & - & 53.1 & 18.6 & - & 178.6 \\
\hline$A(23, X)$ & 122.6 & 8.07 & 4.22 & 1.40 & - & 53.0 & 18.6 & - & 178.8 \\
\hline$K(24, X)$ & 120.1 & 8.09 & 4.22 & 1.80 & 1.46 & 57.2 & 32.7 & 24.9 & 177.3 \\
\hline $\mathrm{A}(25, \mathrm{X})$ & 123.9 & 8.11 & 4.20 & 1.40 & - & 53.2 & 18.5 & - & 178.4 \\
\hline$A(26, X)$ & 122.6 & 8.15 & 4.21 & 1.40 & - & 53.1 & 18.6 & - & 178.3 \\
\hline $\mathbf{A}(27, X)$ & 122.6 & 8.07 & 4.22 & 1.40 & - & 53.0 & 18.6 & - & 178.3 \\
\hline$K(28, X)$ & 120.1 & 8.10 & 4.22 & 1.80 & 1.46 & 56.8 & 32.8 & 24.9 & 176.8 \\
\hline $\mathrm{A}(29, \mathrm{X})$ & 124.1 & 8.13 & 4.22 & 1.39 & - & 52.8 & 19.1 & - & 177.8 \\
\hline $\mathbf{A}(30, X)$ & 122.5 & 8.12 & 4.22 & 1.40 & - & 53.2 & 18.8 & - & 177.7 \\
\hline$Q(31, X)$ & 118.8 & 8.10 & 4.13 & 2.19 & 1.90 & 55.9 & 29.5 & 33.8 & 175.7 \\
\hline $\mathbf{F}(32, X)$ & 120.6 & 8.20 & 4.60 & 3.17 / 2.99 & - & 57.9 & 39.5 & - & 176.1 \\
\hline$G(33, X)$ & 110.6 & 8.24 & 3.85 & - & - & 45.0 & - & - & 173.2 \\
\hline $\mathrm{A}(1,24)$ & 129.4 & 8.42 & 4.58 & 1.34 & - & 50.4 & 18.2 & - & 175.4 \\
\hline$P(2,24)$ & 135.1 & - & 4.38 & $2.25 / 1.90$ & $2.03 / 2.26$ & 63.4 & 32.1 & 27.4 & 177.6 \\
\hline$G(3,24)$ & 109.1 & 8.43 & 3.92 & - & - & 45.2 & - & - & 174.1 \\
\hline
\end{tabular}




\begin{tabular}{lccccccccc}
$\mathbf{V ( 4 , 2 4 )}$ & 119.3 & 7.96 & 4.12 & 2.08 & 0.91 & 62.5 & 32.6 & 20.9 & 176.7 \\
$\mathbf{G ( 5 , 2 4 )}$ & 112.6 & 8.52 & 3.93 & - & - & 45.2 & - & - & 173.7 \\
$\mathbf{V ( 6 , 2 4 )}$ & 119.1 & 7.92 & 4.10 & 2.02 & 0.87 & 61.8 & 33.0 & 20.4 & 175.5 \\
& & & & & & & & & \\
$\mathbf{E ( 1 , X 2 )}$ & 123.6 & 8.67 & 4.19 & 2.28 & 2.01 & 57.8 & 29.5 & 36.3 & 177.5 \\
$\mathbf{A ( 2 , X 2 )}$ & 124.5 & 8.29 & 4.21 & 1.41 & - & 53.6 & 18.7 & - & 179.2 \\
$\mathbf{Q ( 3 , X 2 )}$ & 119.3 & 8.31 & 4.21 & 2.39 & 2.07 & 57.3 & 28.8 & 34.0 & 177.3 \\
$\mathbf{A ( 4 , X 2 )}$ & 124.2 & 8.24 & 4.20 & 1.42 & - & 53.8 & 18.5 & - & 179.2 \\
$\mathbf{A ( 5 , X 2 )}$ & 122.5 & 8.17 & 4.21 & 1.42 & - & 53.8 & 18.5 & - & 179.1 \\
\hline
\end{tabular}




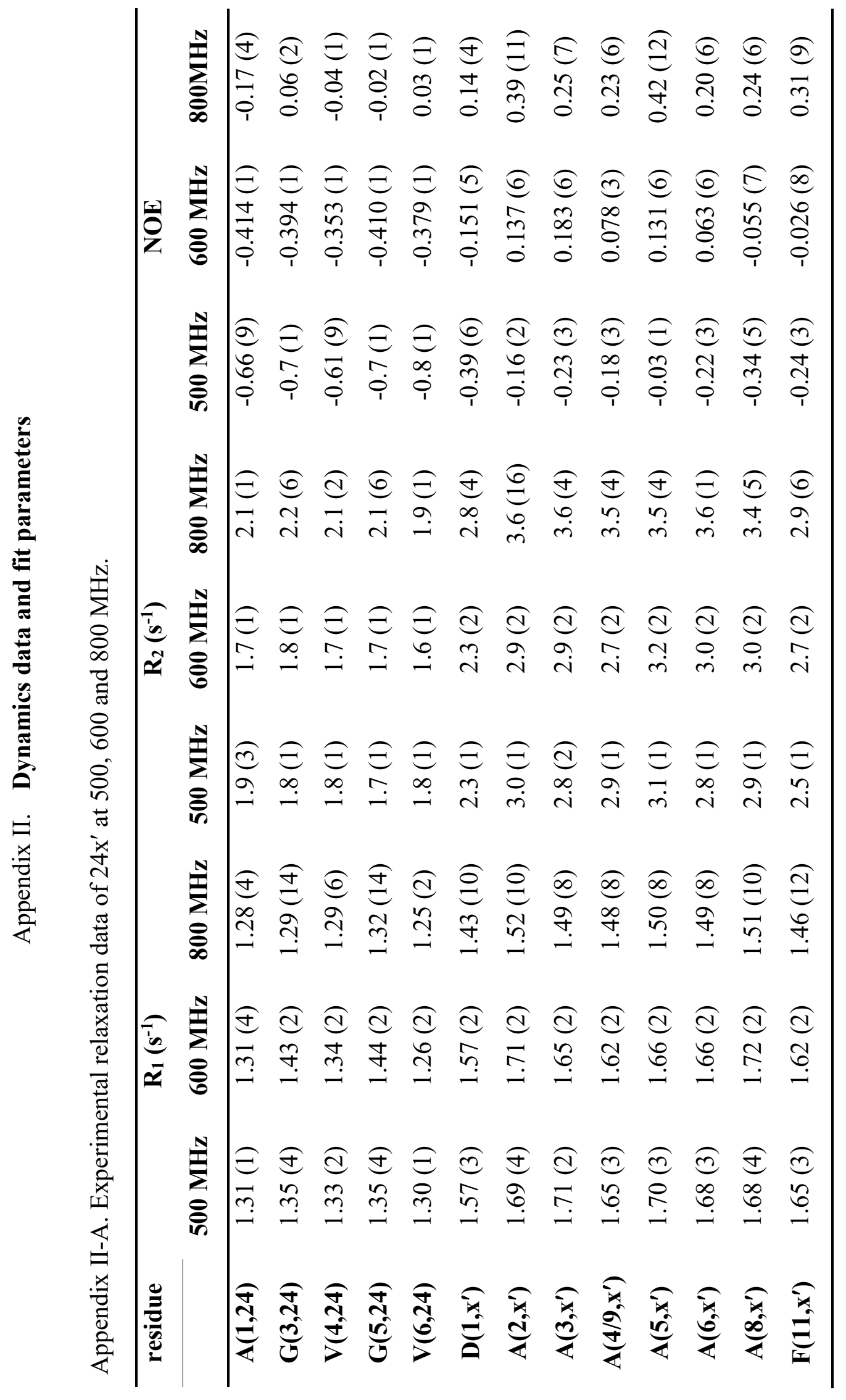




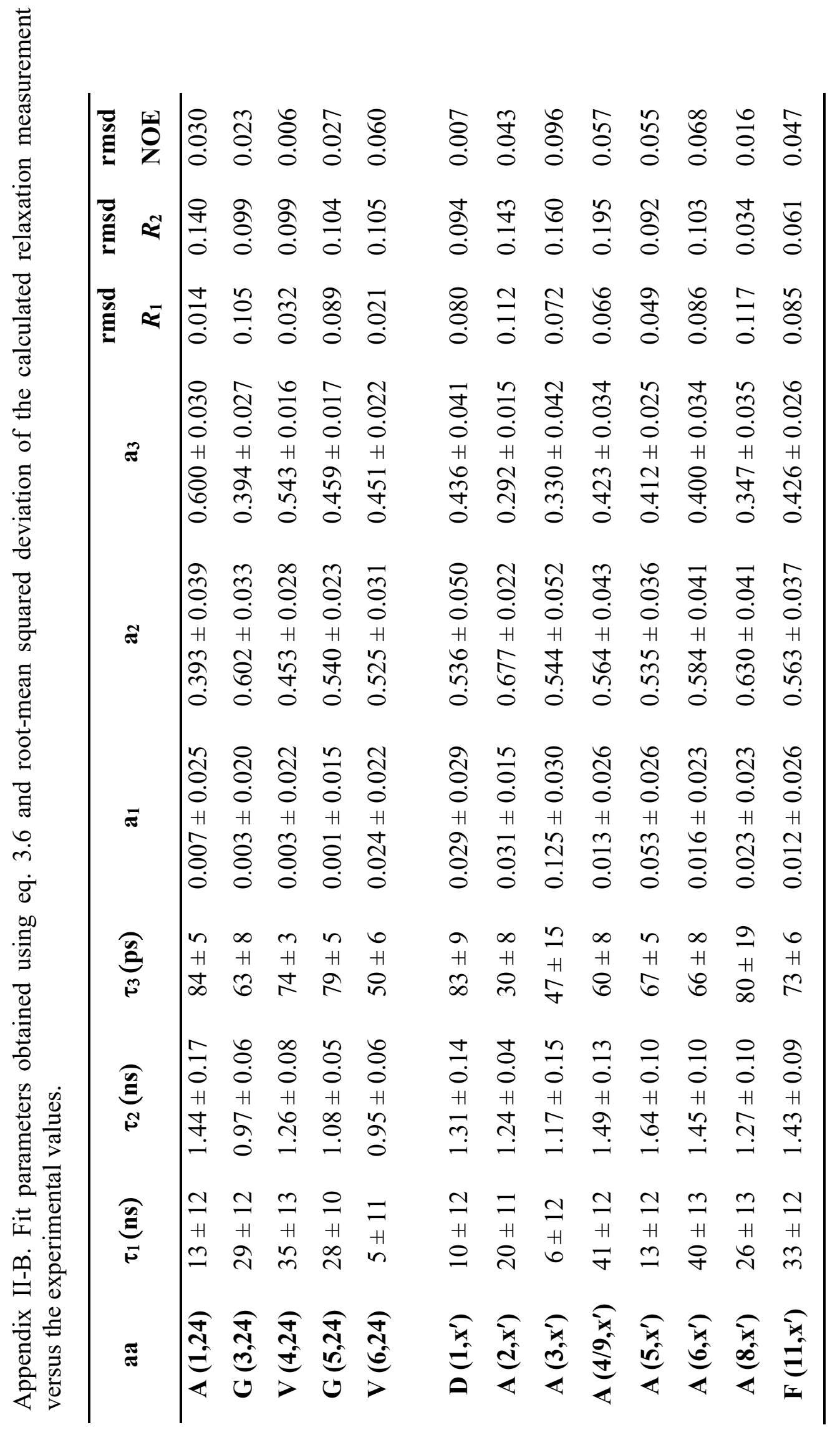




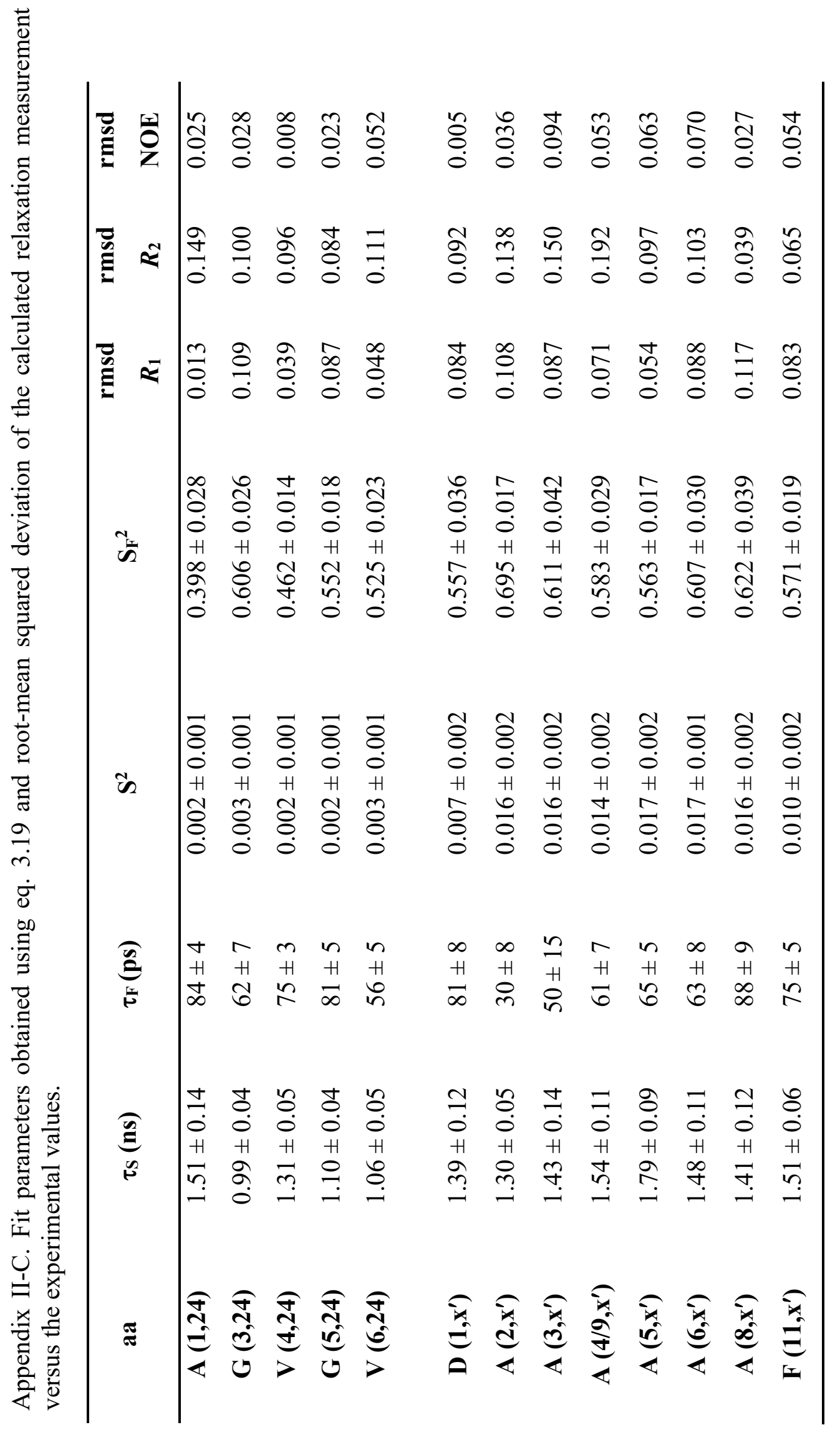




\section{CURRICULUM VITAE}

Ma. Faye Charmagne A. Carvajal

Department of Chemistry, University of Louisville

faye.carvajal@louisville.edu

\section{Education}

University of Louisville

Ph.D Chemistry Candidate

May 2018-present

M.S. Chemistry

May 2018

University of Philippines

B.S. Chemistry

April 2010

\section{Research Experience}

Graduate Research:

Dissertation Title: Investigating the Disorder and Compaction of Designed Minielastins using Nuclear Magnetic Resonance

- Bacterial protein expression of labelled and unlabelled minielastin constructs for NMR analysis

- Performed 2D and 3D NMR experiments using Varian NMR spectrometer and analysis of spectra using NMRPipe and SPARKY

- Data analysis of diffusion, spin relaxation and high-pressure NMR experiments using Topspin, Igor Pro and MATLAB

- Collaboration with other research lab in experimentations and publications

\section{Undergraduate Research:}

Thesis Title: Phytochemical Screening and Characterization of the Antibacterial Activity of Modified Diospyros blancoi Extract

- Ethanolic extraction of dried Diospyros blancoi leaves

- Chromatographic separation and phytochemical analysis of extracts 


\section{Teaching and Work Experience}

Teaching Assistant, General Chemistry I and II January 2018 - present

Teaching Assistant, Biochemistry Laboratory August - December 2017 Graduate Research Assistant January - December 2016

Teaching Assistant, Chemistry for Health Professionals

Teaching Assistant, Organic Chemistry Laboratory January - December 2015 August - December 2014

Department of Chemistry, University of Louisville

- Prepare lecture summary and problem sets for general chemistry I and II recitation classes to boost understanding of chemistry concepts

- Review of chemistry concepts for organic and biochemistry lab classes

- Prepare reagents and supervise students in performing experiments for organic and biochemistry lab classes

- Use of online platform such as Blackboard and Microsoft Teams for online instructions

RM-Process Chemist

July 2011 - July 2014

Unilever Philippines Quality Assurance Department

- Performed quality control testing on personal care and home care raw materials to ensure compliance in specifications of materials prior to production use

- Performed routine analyses of finished goods and environmental samples

- Performed air quality sampling and enzyme analysis

- Supported supply chain and R\&D needs for RM supplier approval by conducting RM analysis

- Supervising and training undergraduate interns

\section{PRESENTATIONS AND CONFERENCES}

Carvajal, M.F.C.A., Greenland, K.N, Preston, J.M., Koder, R.L, Wittebort, R.J. NMR Studies of Structure and Dynamics of Minielastin. Poster Presentation at the $32^{\text {nd }}$ Gibbs Conference for Biological Thermodynamics, Makanda, IL 10/06/201810/09/2018. 
Carvajal, M.F.C.A., Greenland, K.N, Preston, J.M., Koder, R.L, Wittebort, R.J. NMR Studies of Secondary Structure and Compaction of Minielastin. Poster Presentation at the Graduate Student Regional Research Conference, University of Louisville $03 / 02 / 2018$.

Carvajal, M.F.C.A., Greenland, K.N, Preston, J.M., Koder, R.L, Wittebort, R.J. NMR Studies of Secondary Structure and Compaction of Minielastin. Poster Presentation at the 2018 Biomolecular Solid-State NMR Winter School, Stowe, Vermont 01/07/2018-01/12/2018.

Carvajal, M.F.C.A., Greenland, K.N, Preston, J.M., Koder, R.L, Wittebort, R.J. NMR Studies of Secondary Structure and Compaction of Minielastin. Poster Presentation at the $2^{\text {nd }}$ Annual Gateway NMR Meeting, The Ohio State University 11/04/2017.

Carvajal, M.F.C.A., Greenland, K.N, Preston, J.M., Koder, R.L, Wittebort, R.J. NMR Studies of Secondary Structure and Compaction of Minielastin. Poster Presentation at the Research!Louisville 2017, University of Louisville 09/12/2017.

Carvajal, M.F.C.A., Greenland, K.N, Preston, J.M., Koder, R.L, Wittebort, R.J. NMR Studies of Secondary Structure and Compaction of Minielastin. Poster Presentation at the Gordon Research Conference on Elastin, Elastic Fibers \& Microfibrils, University of New England in Biddeford, ME 7/30/2017-08/04/2017.

Carvajal, M.F.C.A., Greenland, K.N, Preston, J.M., Koder, R.L, Wittebort, R.J. NMR Studies of Secondary Structure and Compaction of Minielastin. Poster Presentation at the Gordon Research Seminar on Elastin, Elastic Fibers \& Microfibrils, University of New England in Biddeford, ME 7/29/2017-07/30/2017.

\section{Publications}

Greenland, K. N., Carvajal, M. F. C. A., Preston, J. M., Ekblad, S., Dean, W. L., Chiang, J. Y., Koder, R.L., Wittebort, R. J. (2018). Order, Disorder, and Temperature-Driven Compaction in a Designed Elastin Protein. Journal of Physical Chemistry B, 122(10), 2725-2736. doi:10.1021/acs.jpcb.7b11596

Carvajal, M. F. C. A., Preston, J. M., Jamhawi, N. M., Sabo, T. M., Aramini, J. M., Wittebort, R. J., Koder, R. L., (2020) Dynamics in Natural and Designed Elastins and Their relation to Elastic Fiber Structure and Recoil. Manuscript submitted for publication. 